\title{
Generic Pressurized Water Reactor Model for SAPHIRE
}

\section{April 2021}

Curtis L Smith

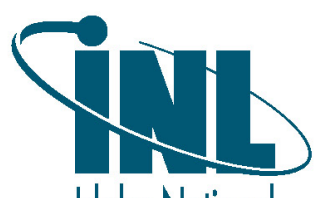

Idaho National

Laboratory 


\section{DISCLAIMER}

This information was prepared as an account of work sponsored by an agency of the U.S. Government. Neither the U.S. Government nor any agency thereof, nor any of their employees, makes any warranty, expressed or implied, or assumes any legal liability or responsibility for the accuracy, completeness, or usefulness, of any information, apparatus, product, or process disclosed, or represents that its use would not infringe privately owned rights. References herein to any specific commercial product, process, or service by trade name, trade mark, manufacturer, or otherwise, does not necessarily constitute or imply its endorsement, recommendation, or favoring by the U.S. Government or any agency thereof. The views and opinions of authors expressed herein do not necessarily state or reflect those of the U.S. Government or any agency thereof. 


\section{Generic Pressurized Water Reactor Model for SAPHIRE}

Curtis L Smith

April 2021

Idaho National Laboratory

Idaho Falls, Idaho 83415

http://www.inl.gov

Prepared for the U.S. Department of Energy

Under DOE Idaho Operations Office

Contract DE-AC07-05ID14517 
This report contains a data dump from a SAPHIRE Generic Pressurized Water Reactor (PWR) model. The intent is to have this information reviewed for export control and classification so that the generic model can be used external to INL. The facility model is not applicable to any existing nuclear reactors.

The Generic PWR model does not represent any existing power plant and was created to support research and training purposes.

Three distinct types of data are used to create a SAPHIRE risk model:

- Event trees representing typical PWR sequences

- Fault trees representing generic system performance

- Basic events (e.g., component failure information) (pages 2-18)

(pages 19-66

(pages 67-100)

Where possible, we have taken information from publicly available documents and referenced that information tagged to a specific item. 
Generic Pressurized Water Reactor (PWR)

\section{EVENT TREE INFORMATION}


EVENT TREE

EQK-BIN1
Hypothetical seismic event tree based upon public NRC document. https://www.nrc.gov/docs/ML1626/ML16264A140.pdf

EQK-BIN2

Hypothetical seismic event tree based upon public NRC document. https://www.nrc.gov/docs/ML1626/ML16264A140.pdf

EQK-BIN3

Hypothetical seismic event tree based upon public NRC document. https://www.nrc.gov/docs/ML1626/ML16264A140.pdf

EQK-BIN4

Hypothetical seismic event tree based upon public NRC document. https://www.nrc.gov/docs/ML1626/ML16264A140.pdf

EQK-BIN4-EXAMPLE Hypothetical seismic event tree based upon public NRC document. https://www.nrc.gov/docs/ML1626/ML16264A140.pdf

EQK-BIN5

Hypothetical seismic event tree based upon public NRC document. https://www.nrc.gov/docs/ML1626/ML16264A140.pdf

EQK-BIN6

Hypothetical seismic event tree based upon public NRC document. https://www.nrc.gov/docs/ML1626/ML16264A140.pdf

EQK-BIN7 Hypothetical seismic event tree based upon public NRC document. https://www.nrc.gov/docs/ML1626/ML16264A140.pdf

FLI-4160VACA Hypothetical internal flooding event tree based upon public NRC training document. https://www.nrc.gov/docs/ML1204/ML12044A209.pdf

FLI-4160VACB Hypothetical internal flooding event tree based upon public NRC training document. https://www.nrc.gov/docs/ML1204/ML12044A209.pdf

FLI-AFW-ROOM Hypothetical internal flooding event tree based upon public NRC training document. https://www.nrc.gov/docs/ML1204/ML12044A209.pdf 


\section{EVENT TREE}

https://www.nrc.gov/docs/ML1204/ML12044A209.pdf

FLI-CCW-ROOMA Hypothetical internal flooding event tree based upon public NRC training document. https://www.nrc.gov/docs/ML1204/ML12044A209.pdf

FLI-CCW-ROOMB Hypothetical internal flooding event tree based upon public NRC training document. https://www.nrc.gov/docs/ML1204/ML12044A209.pdf

FLI-CVC-ROOM Hypothetical internal flooding event tree based upon public NRC training document. https://www.nrc.gov/docs/ML1204/ML12044A209.pdf

FLI-RHR-ROOM Hypothetical internal flooding event tree based upon public NRC training document. https://www.nrc.gov/docs/ML1204/ML12044A209.pdf

FLI-SWS-ROOM Hypothetical internal flooding event tree based upon public NRC training document. https://www.nrc.gov/docs/ML1204/ML12044A209.pdf

FLI-SWS-ROOMA Hypothetical internal flooding event tree based upon public NRC training document. https://www.nrc.gov/docs/ML1204/ML12044A209.pdf

FLI-SWS-ROOMB Hypothetical internal flooding event tree based upon public NRC training document. https://www.nrc.gov/docs/ML1204/ML12044A209.pdf

FRI-AB-AFWAB $\quad$ Hypothetical internal fire event tree based upon public NRC and EPRI training documents.

https://www.nrc.gov/docs/ML1204/ML12044A209.pdf https://www.nrc.gov/docs/ML1025/ML102530294.pdf https://www.nrc.gov/docs/ML1422/ML14226B016.pdf

FRI-AB-CCWBC $\quad$ Hypothetical internal fire event tree based upon public NRC and EPRI training documents.

https://www.nrc.gov/docs/ML1204/ML12044A209.pdf

https://www.nrc.gov/docs/ML1025/ML102530294.pdf

https://www.nrc.gov/docs/ML1422/ML14226B016.pdf

FRI-AB-LOOP

Hypothetical internal fire event tree based upon public NRC and EPRI training documents.

https://www.nrc.gov/docs/ML1204/ML12044A209.pdf

https://www.nrc.gov/docs/ML1025/ML102530294.pdf

https://www.nrc.gov/docs/ML1422/ML14226B016.pdf 


\section{$\underline{\text { EVENT TREE }}$}

FRI-AB-LOOP-DIVA Hypothetical internal fire event tree based upon public NRC and EPRI training documents.

https://www.nrc.gov/docs/ML1204/ML12044A209.pdf https://www.nrc.gov/docs/ML1025/ML102530294.pdf https://www.nrc.gov/docs/ML1422/ML14226B016.pdf

FRI-AB-LOOP-DIVB Hypothetical internal fire event tree based upon public NRC and EPRI training documents.

https://www.nrc.gov/docs/ML1204/ML12044A209.pdf https://www.nrc.gov/docs/ML1025/ML102530294.pdf https://www.nrc.gov/docs/ML1422/ML14226B016.pdf

FRI-AB-RHRA Hypothetical internal fire event tree based upon public NRC and EPRI training documents.

https://www.nrc.gov/docs/ML1204/ML12044A209.pdf https://www.nrc.gov/docs/ML1025/ML102530294.pdf https://www.nrc.gov/docs/ML1422/ML14226B016.pdf

FRI-AB-SIS Hypothetical internal fire event tree based upon public NRC and EPRI training documents.

https://www.nrc.gov/docs/ML1204/ML12044A209.pdf https://www.nrc.gov/docs/ML1025/ML102530294.pdf https://www.nrc.gov/docs/ML1422/ML14226B016.pdf

FRI-AB-SLOCA Hypothetical internal fire event tree based upon public NRC and EPRI training documents.

https://www.nrc.gov/docs/ML1204/ML12044A209.pdf https://www.nrc.gov/docs/ML1025/ML102530294.pdf https://www.nrc.gov/docs/ML1422/ML14226B016.pdf

FRI-MCR Hypothetical internal fire event tree based upon public NRC and EPRI training documents.

https://www.nrc.gov/docs/ML1204/ML12044A209.pdf https://www.nrc.gov/docs/ML1025/ML102530294.pdf https://www.nrc.gov/docs/ML1422/ML14226B016.pdf

FRI-SWS-BLD Hypothetical internal fire event tree based upon public NRC and EPRI training documents.

https://www.nrc.gov/docs/ML1204/ML12044A209.pdf https://www.nrc.gov/docs/ML1025/ML102530294.pdf https://www.nrc.gov/docs/ML1422/ML14226B016.pdf 
Generic Pressurized Water Reactor (PWR)

EVENT TREE

HCN-BIN1 Hypothetical hurricane event tree based upon public NRC training document. https://www.nrc.gov/docs/ML1204/ML12044A209.pdf

HCN-BIN2 Hypothetical hurricane event tree based upon public NRC training document. https://www.nrc.gov/docs/ML1204/ML12044A209.pdf

HCN-BIN3 Hypothetical hurricane event tree based upon public NRC training document. https://www.nrc.gov/docs/ML1204/ML12044A209.pdf

HCN-BIN4 Hypothetical hurricane event tree based upon public NRC training document. https://www.nrc.gov/docs/ML1204/ML12044A209.pdf

HWD-96MPH Hypothetical high winds event tree based upon public NRC training document. https://www.nrc.gov/docs/ML1204/ML12044A209.pdf

ISL-RHR-CL Hypothetical ISLOCA event tree based upon a simplified version of the logic found in NUREG/CR-5604

https://www.osti.gov/servlets/purl/5282295

ISL-RHR-HL Hypothetical ISLOCA event tree based upon a simplified version of the logic found in NUREG/CR-5604 https://www.osti.gov/servlets/purl/5282295

L4160ACA Hypothetical upset condition leading to a transient event tree, similar to the event tree in Figure 2-2 from NUREG-CR-5465.

L4160ACB Hypothetical upset condition leading to a transient event tree, similar to the event tree in Figure 2-2 from NUREG-CR-5465.

LLOCA Hypothetical large break LOCA event tree, similar to the event tree in Figure 2-7 from NUREG-CR-5465.

LOCCW Hypothetical loss of ccw event tree.

LODCA Hypothetical loss of DC bus A event tree.

LODCB Hypothetical loss of DC bus B event tree.

LOMFW Hypothetical loss of feedwater event tree. 


\section{EVENT TREE}

LOOPGR Hypothetical loss of offsite power event tree, similar to the event tree in Figure 2-4 from NUREG-CR-5465.

LOOPPC Hypothetical loss of offsite power event tree, similar to the event tree in Figure 2-4 from NUREG-CR-5465.

LOOPSC Hypothetical loss of offsite power event tree, similar to the event tree in Figure 2-4 from NUREG-CR-5465.

LOOPWR

Hypothetical loss of offsite power event tree, similar to the event tree in Figure 2-4 from NUREG-CR-5465.

LSSB

Hypothetical large steam line break event tree.

MLOCA Hypothetical medium break LOCA event tree, similar to the event tree in Figure 2-6 from NUREG-CR-5465.

SGTR

Hypothetical steam generator tube rupture event tree.

SLOCA

Hypothetical small break LOCA event tree, similar to the event tree in Figure 2-5 from NUREG-CR-5465.

TOR-BIN1

Hypothetical tornado event tree based upon public NRC training document. https://www.nrc.gov/docs/ML1204/ML12044A209.pdf

TOR-BIN2 Hypothetical tornado event tree based upon public NRC training document. https://www.nrc.gov/docs/ML1204/ML12044A209.pdf

TOR-BIN3 Hypothetical tornado event tree based upon public NRC training document. https://www.nrc.gov/docs/ML1204/ML12044A209.pdf

TRANS

Hypothetical upset condition leading to a transient event tree, similar to the event tree in Figure 2-2 from NUREG-CR-5465.

XLOCA

Hypotetical excessive LOCA event tree. 


\section{EQK-BIN1}

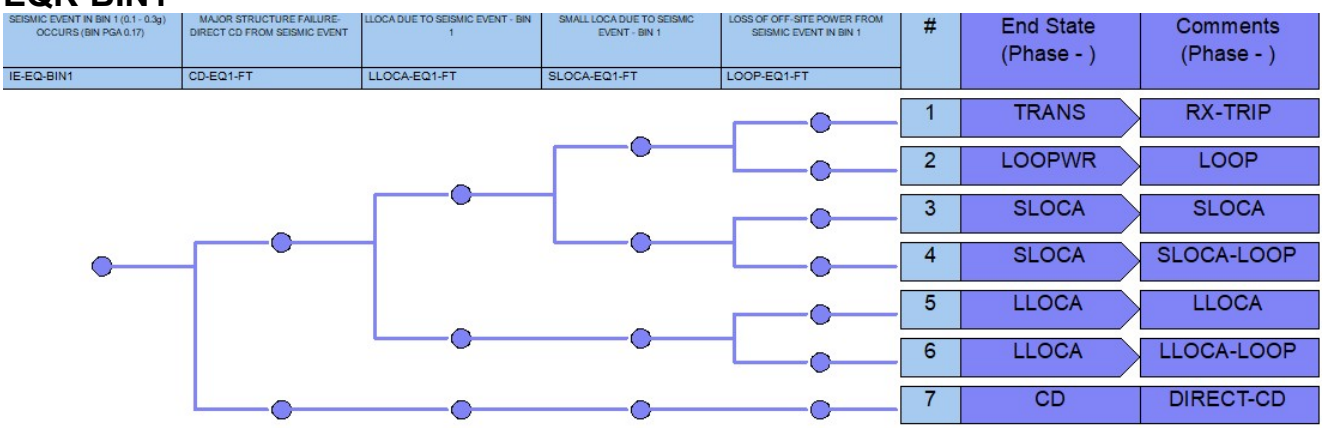

\section{EQK-BIN2}

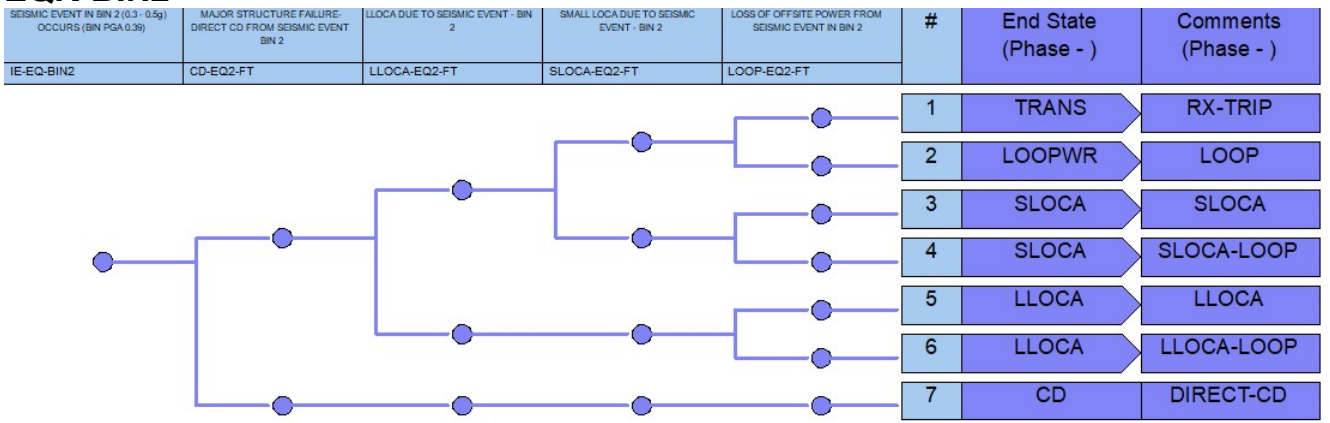

\section{EQK-BIN3}

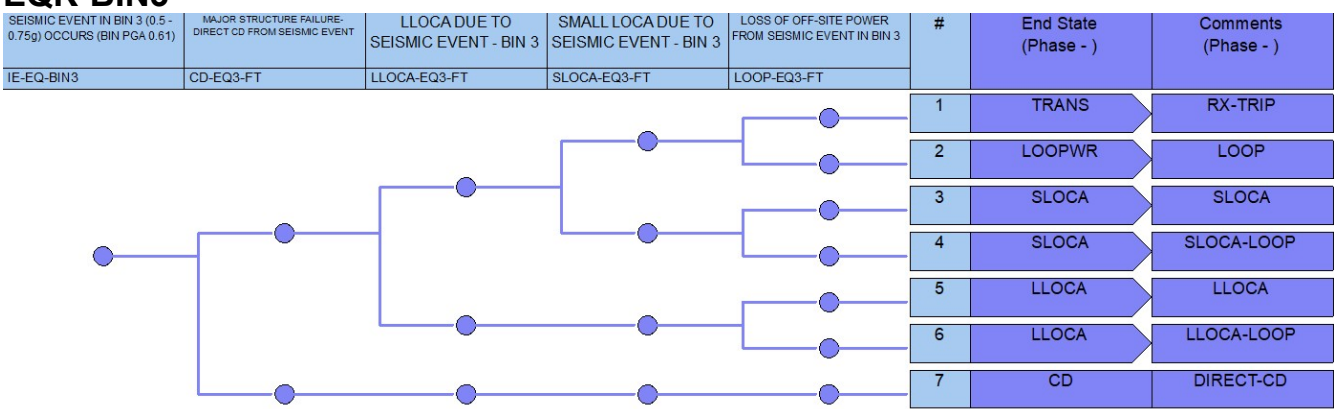

\section{EQK-BIN4}

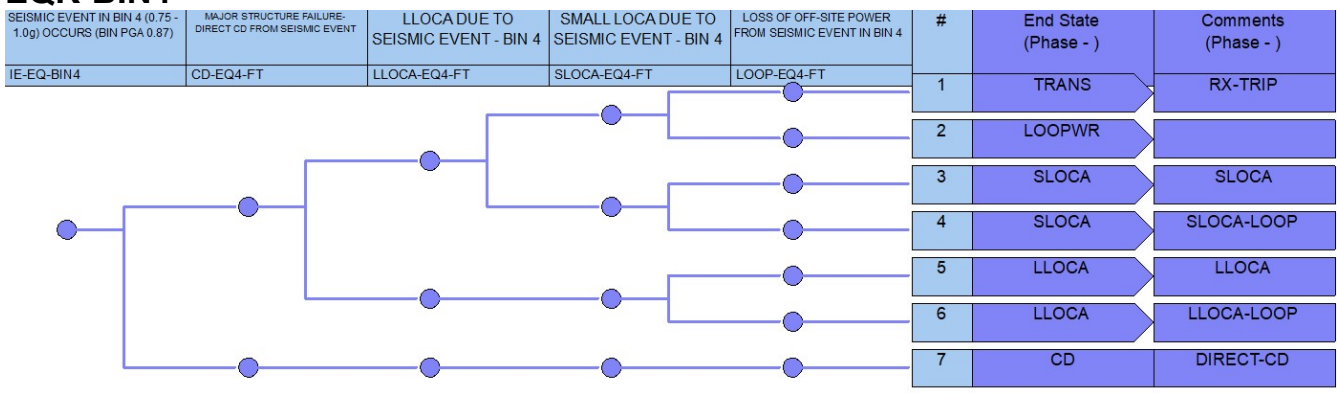

\section{EQK-BIN4-EXAMPLE}




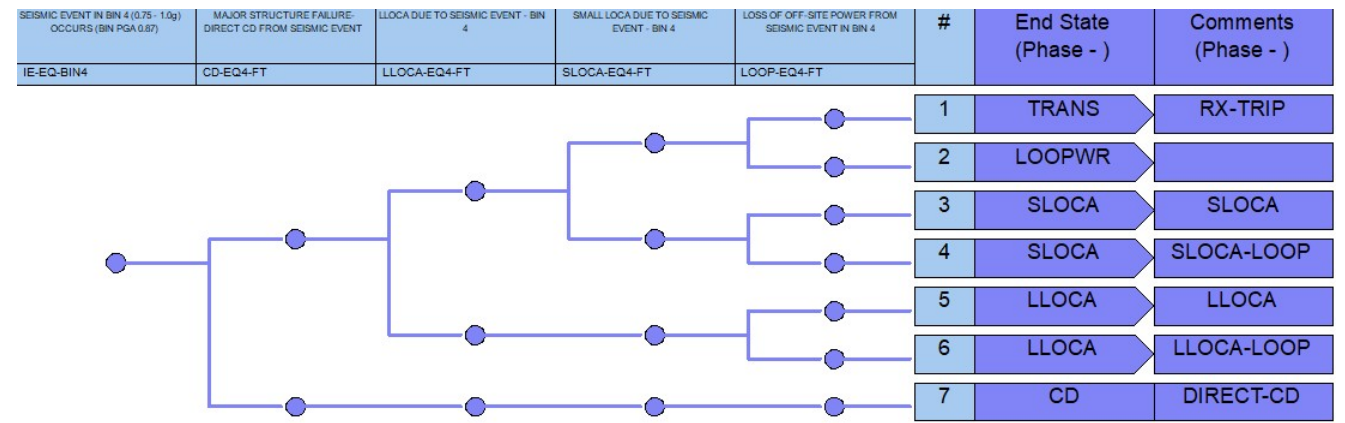

\section{EQK-BIN5}

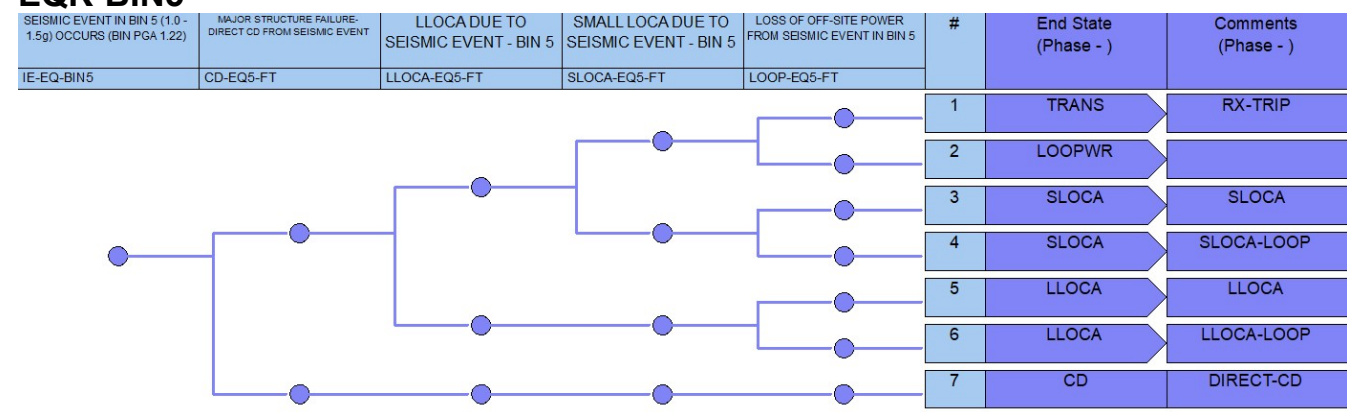

\section{EQK-BIN6}

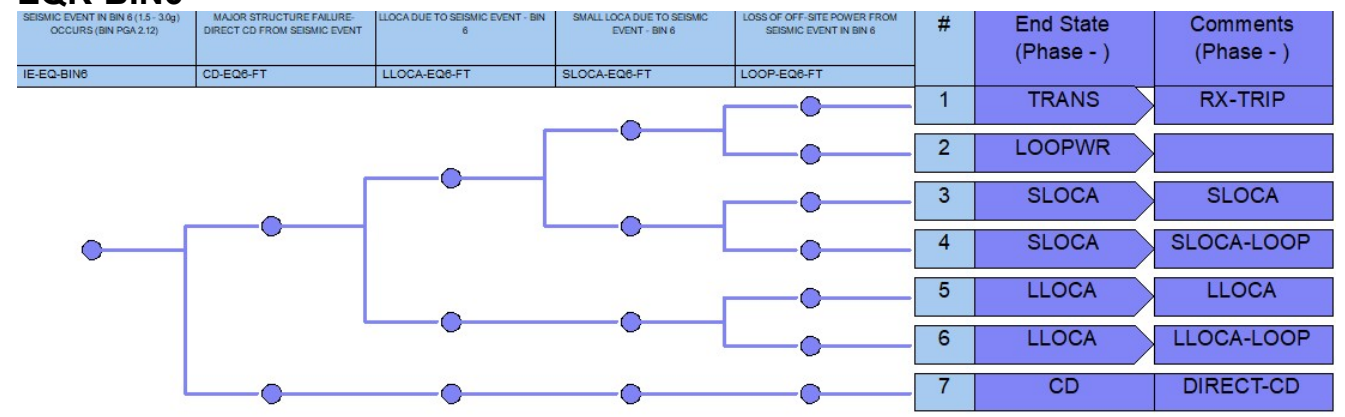

\section{EQK-BIN7}

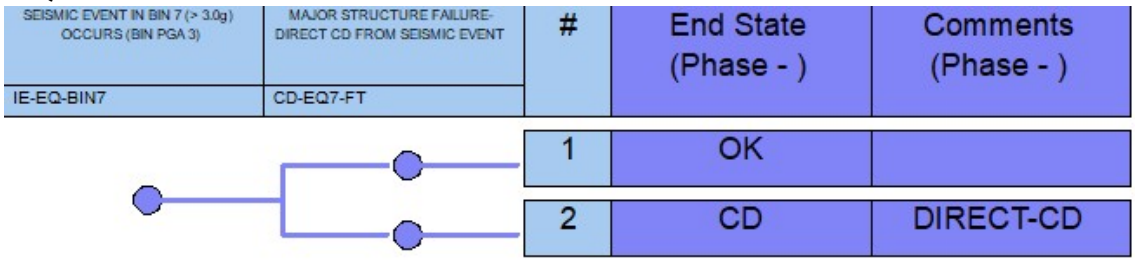

\section{FLI-4160VACA}

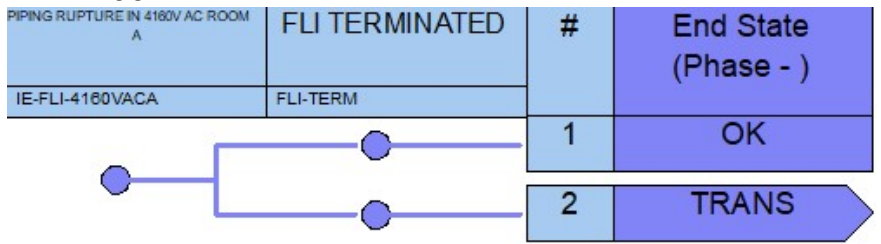

\section{FLI-4160VACB}

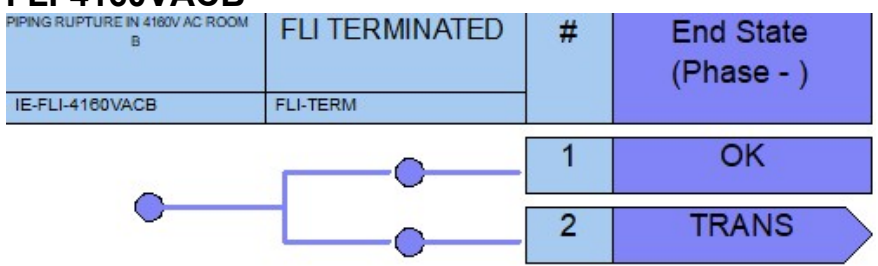

\section{FLI-AFW-ROOM}


Generic Pressurized Water Reactor (PWR)

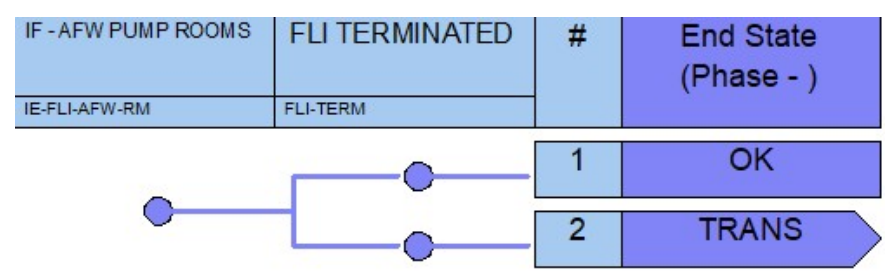

\section{FLI-CCW-ROOM}

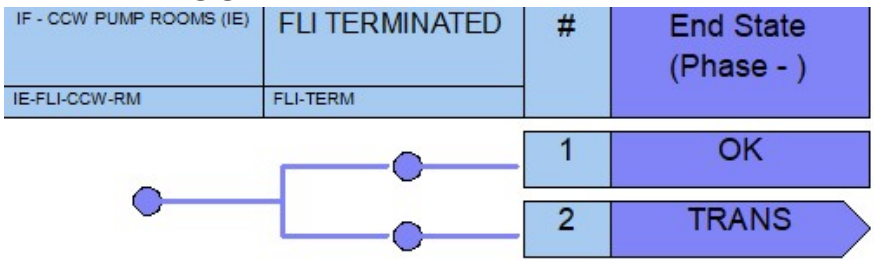

FLI-CCW-ROOMA

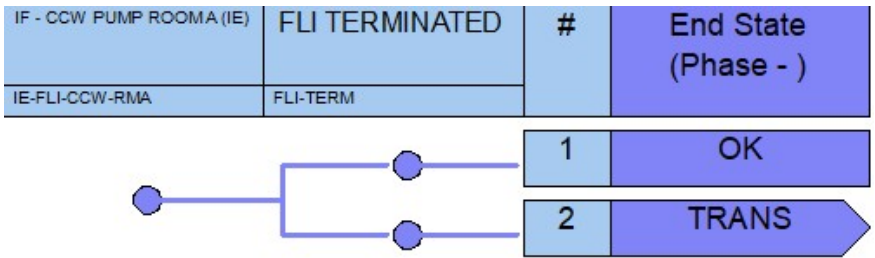

FLI-CCW-ROOMB

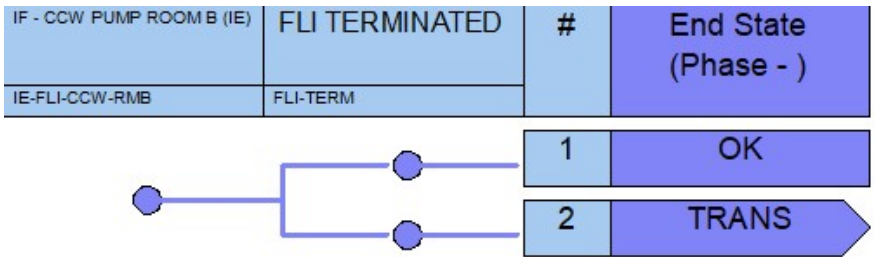

\section{FLI-CVC-ROOM}

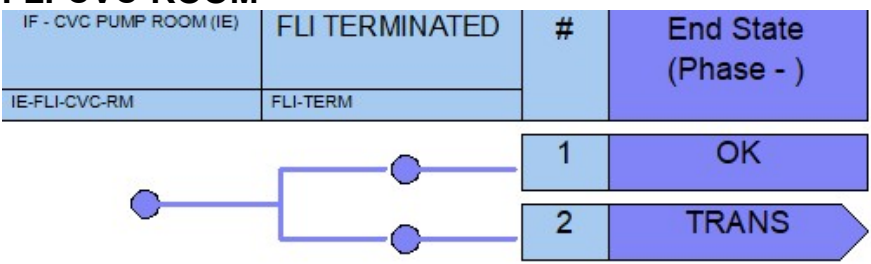

\section{FLI-RHR-ROOM}

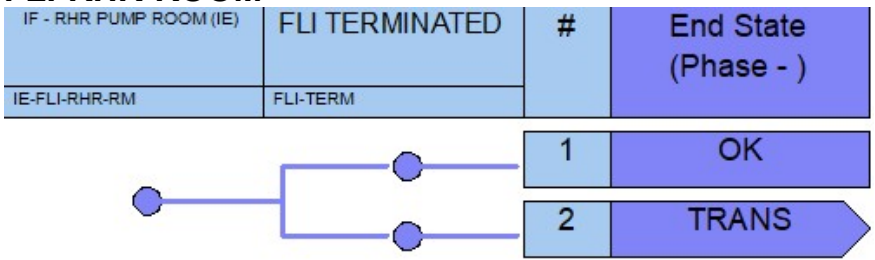

\section{FLI-SWS-ROOM}

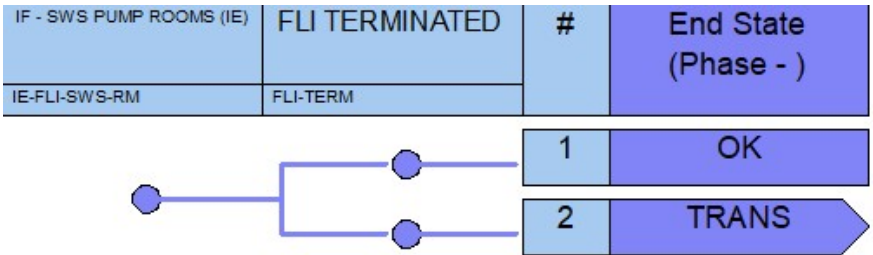

FLI-SWS-ROOMA

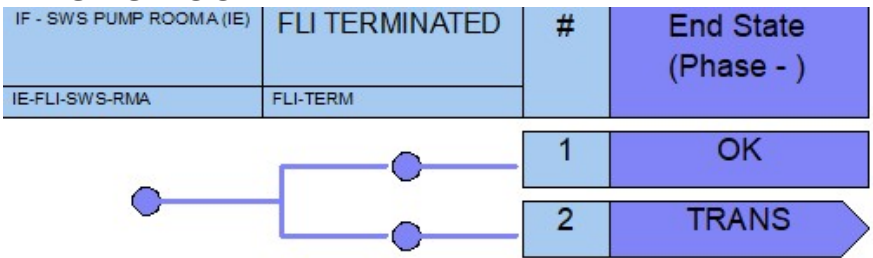


Generic Pressurized Water Reactor (PWR)

FLI-SWS-ROOMB

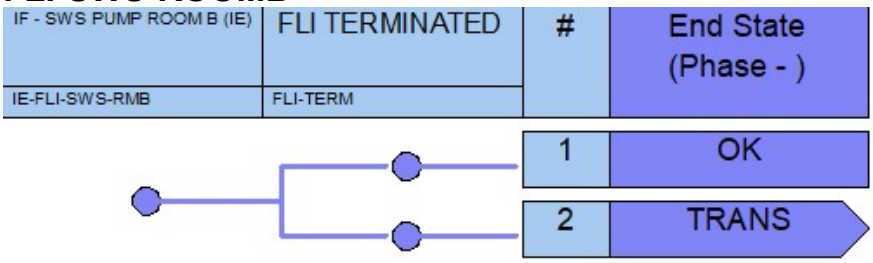

FRI-AB-AFWAB

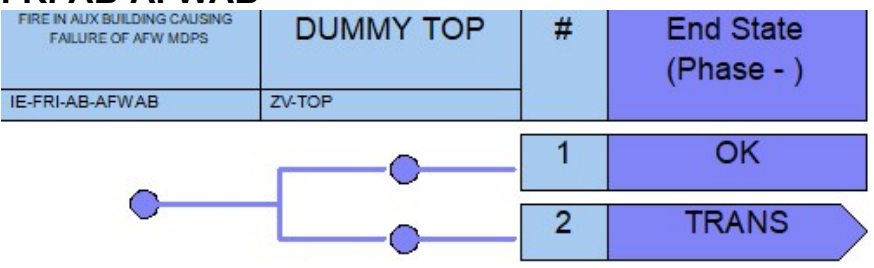

FRI-AB-CCWBC

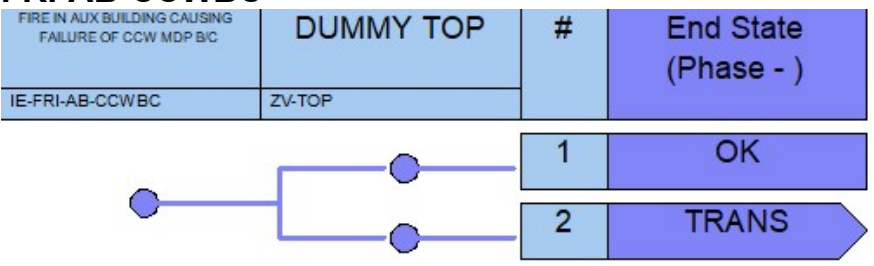

FRI-AB-LOOP

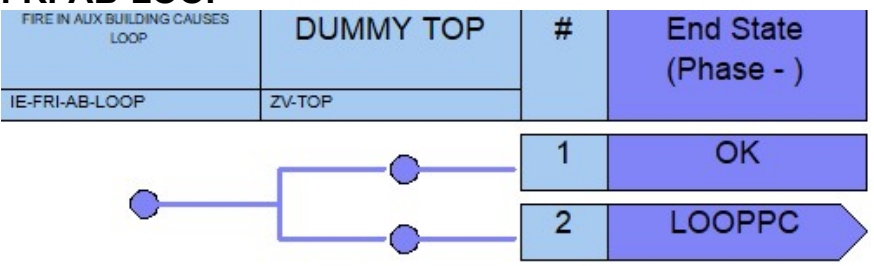

FRI-AB-LOOP-DIVA

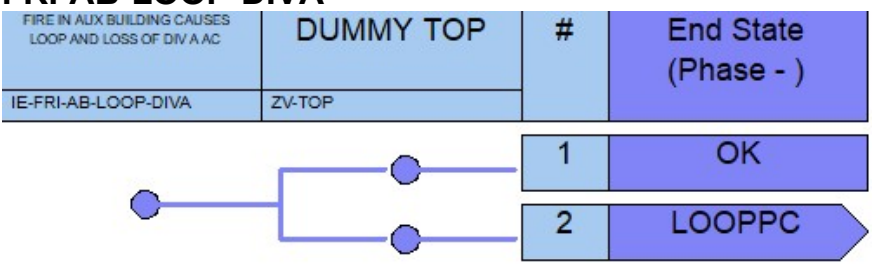

FRI-AB-LOOP-DIVB

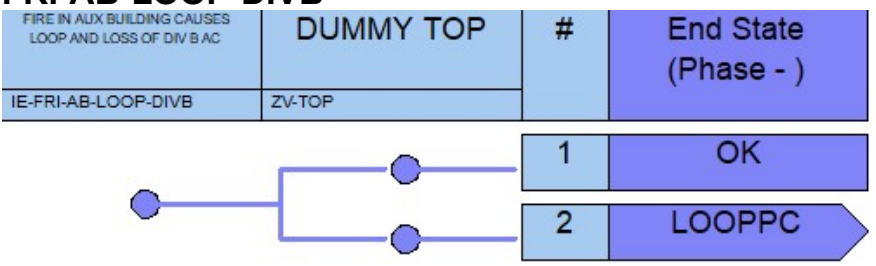

FRI-AB-RHRA

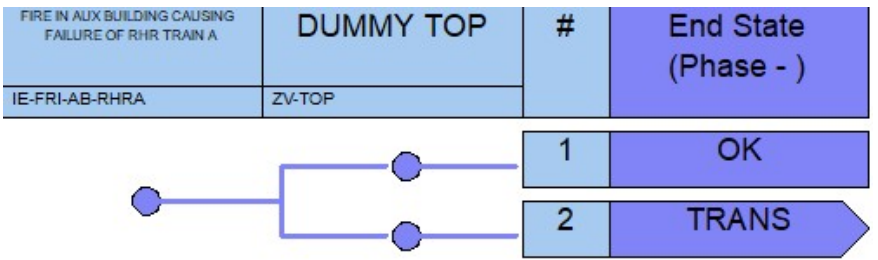

FRI-AB-SIS 
Generic Pressurized Water Reactor (PWR)

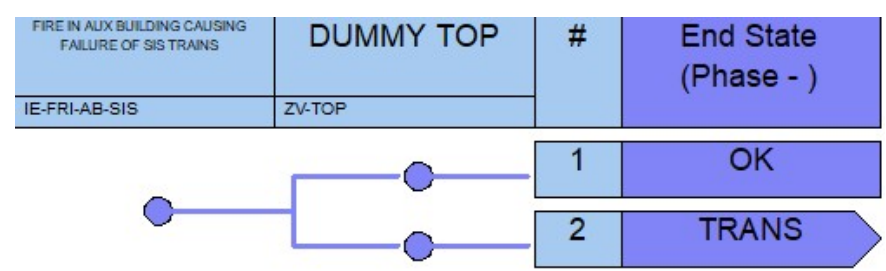

\section{FRI-AB-SLOCA}

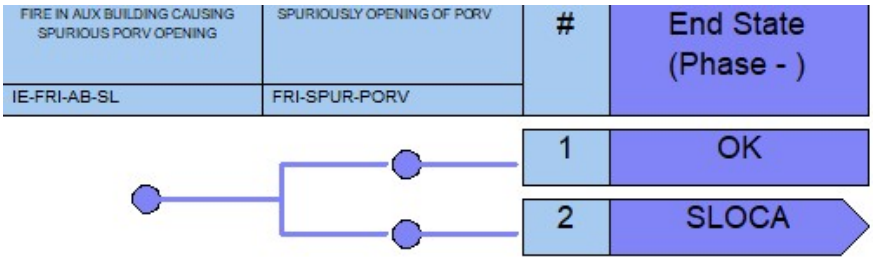

\section{FRI-MCR}

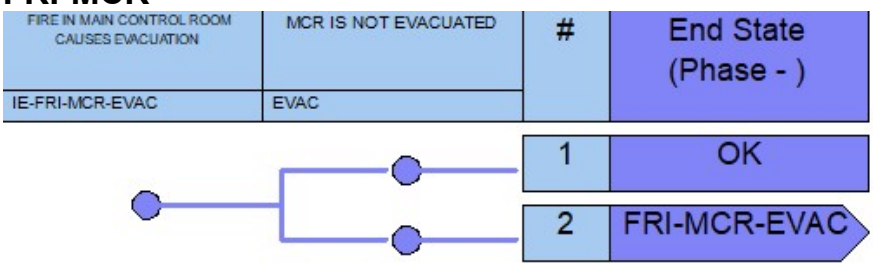

\section{FRI-SWS-BLD}

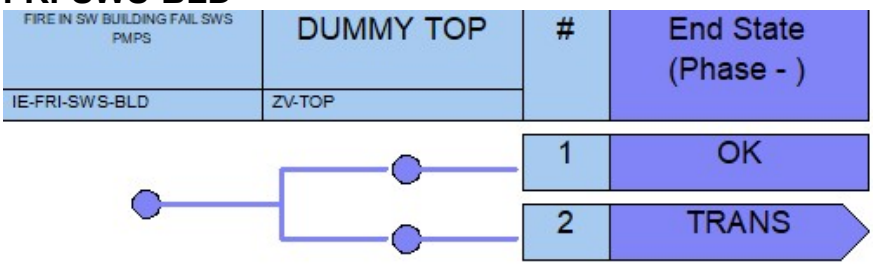

\section{HCN-BIN1}

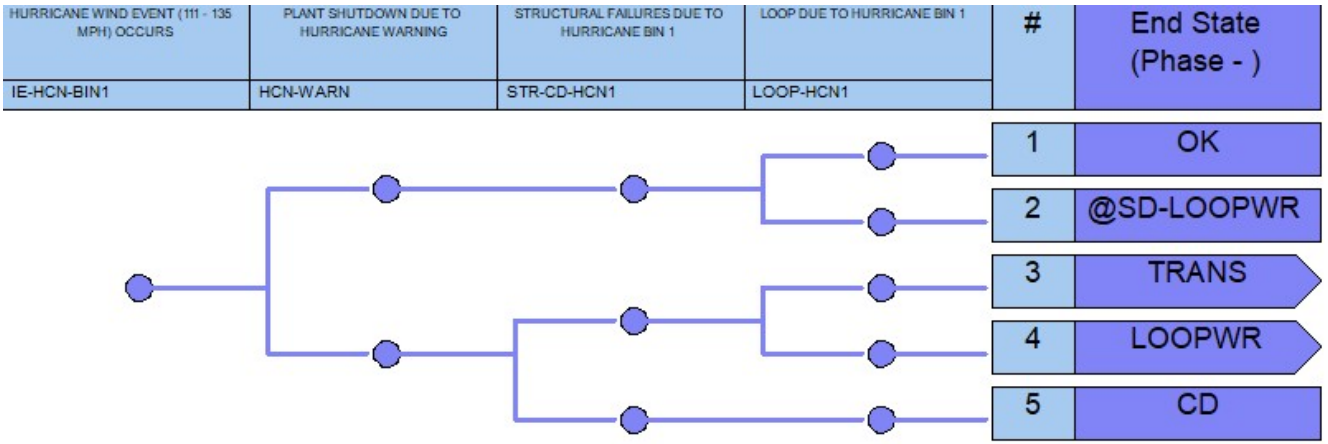

\section{HCN-BIN2}

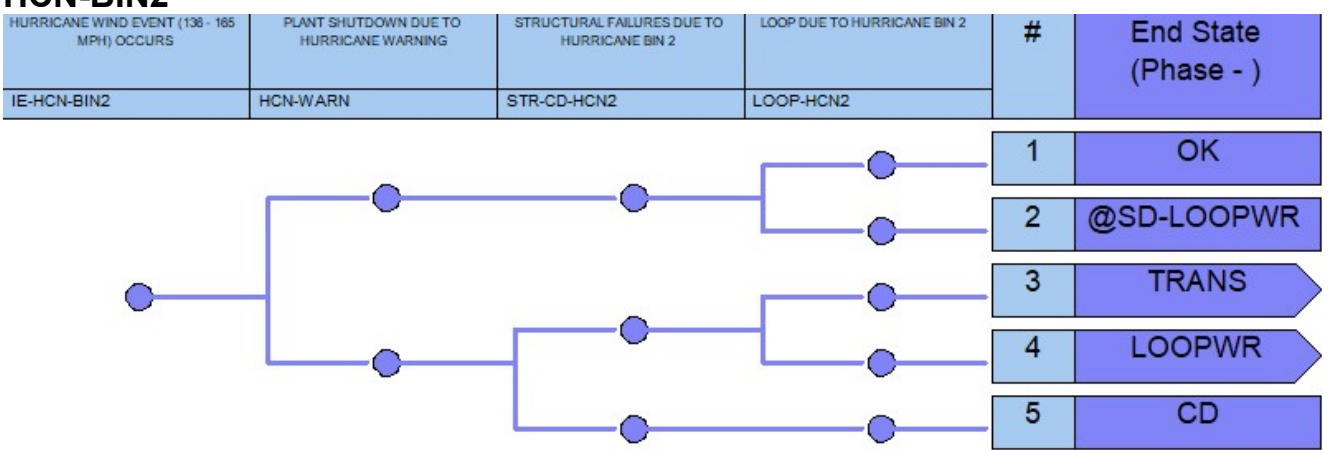

HCN-BIN3 
Generic Pressurized Water Reactor (PWR)

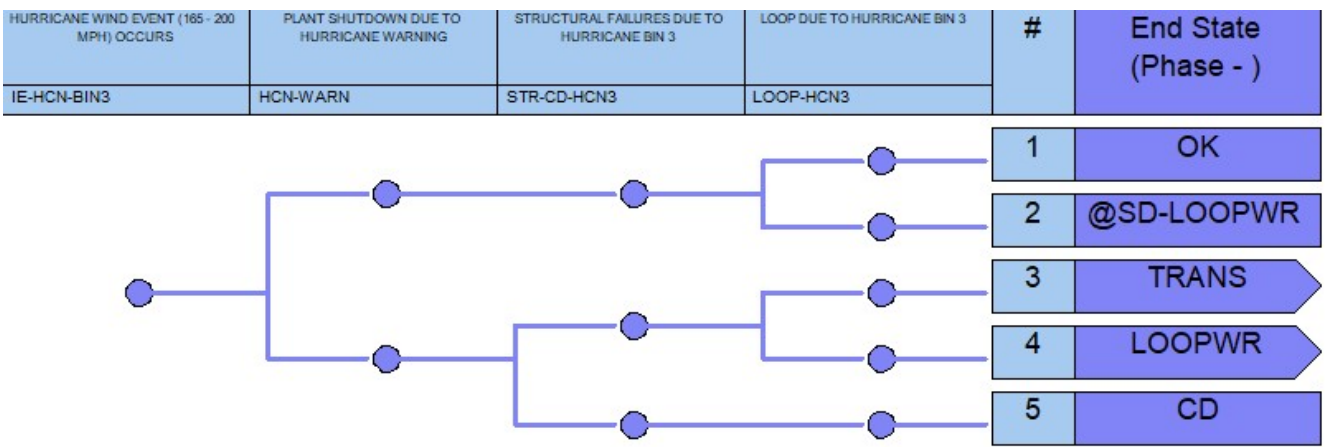

\section{HCN-BIN4}

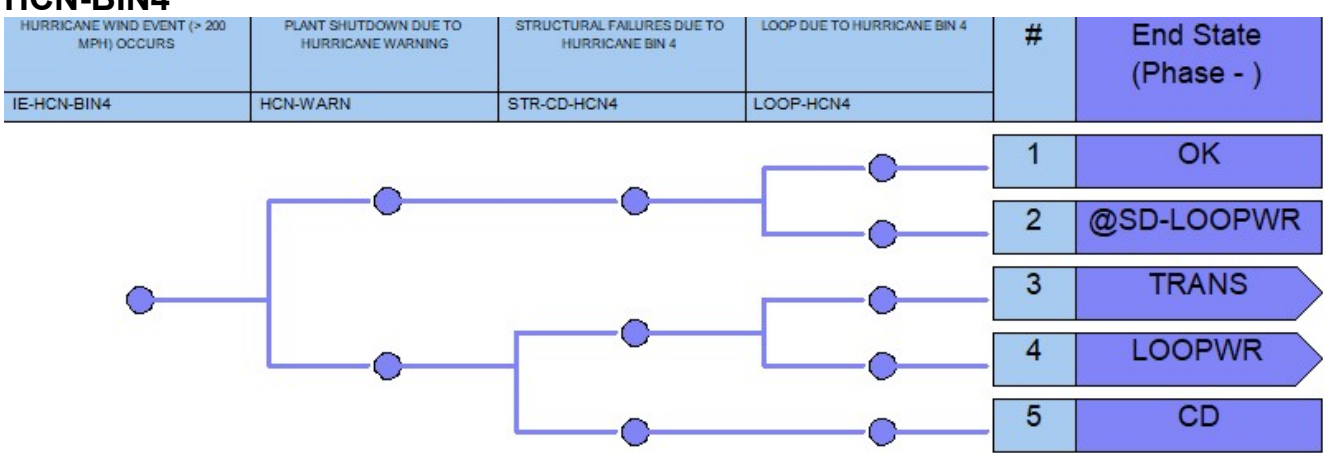

\section{HWD-96MPH}

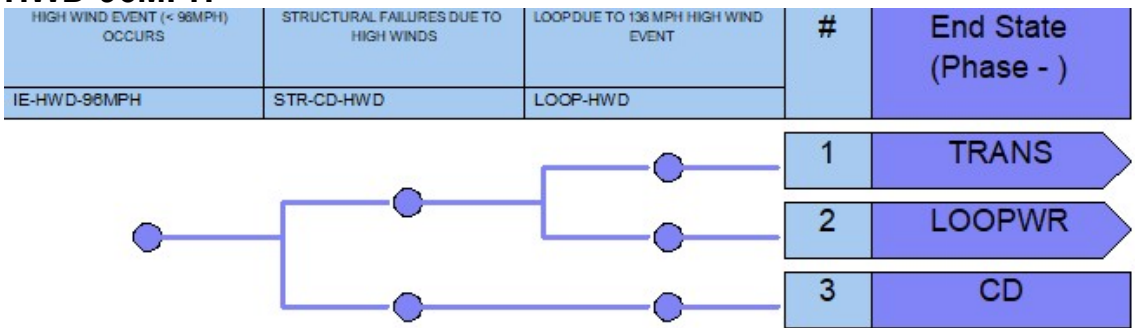

\section{ISL-RHR-CL}

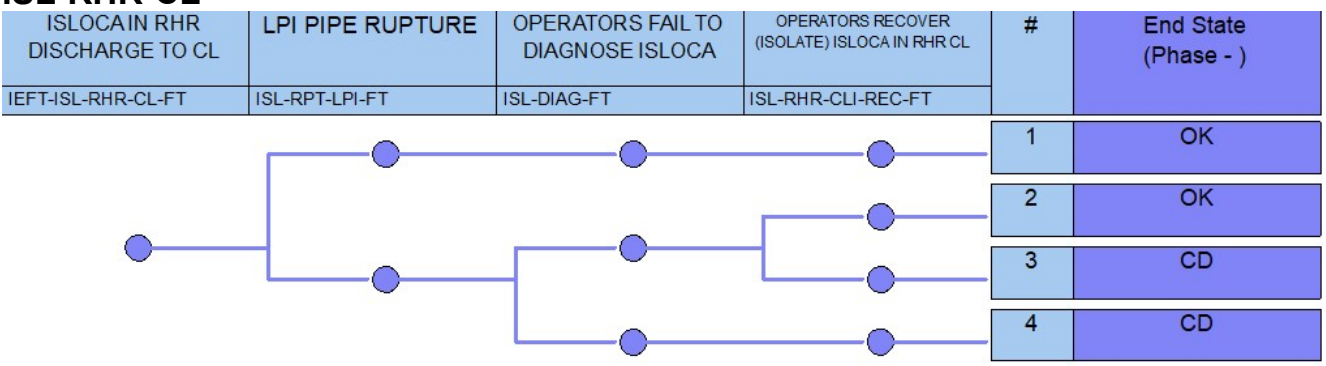

ISL-RHR-HL

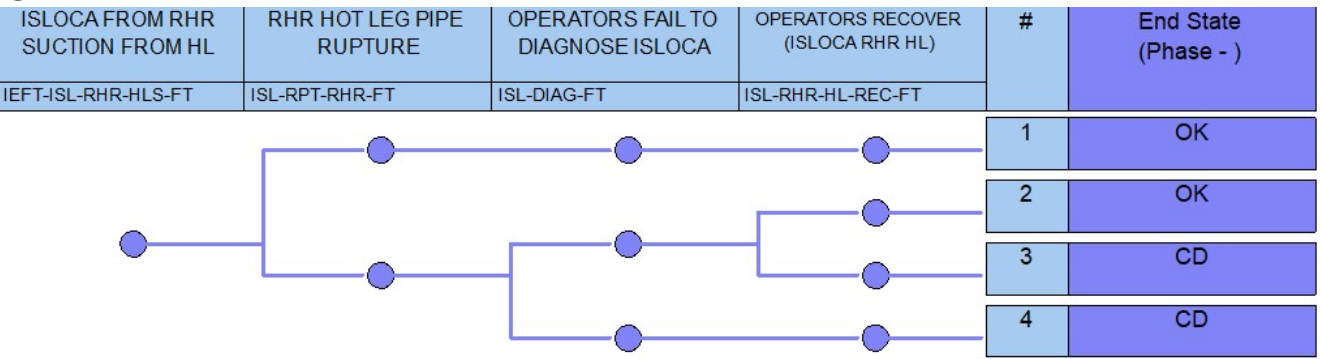

\section{L4160ACA}




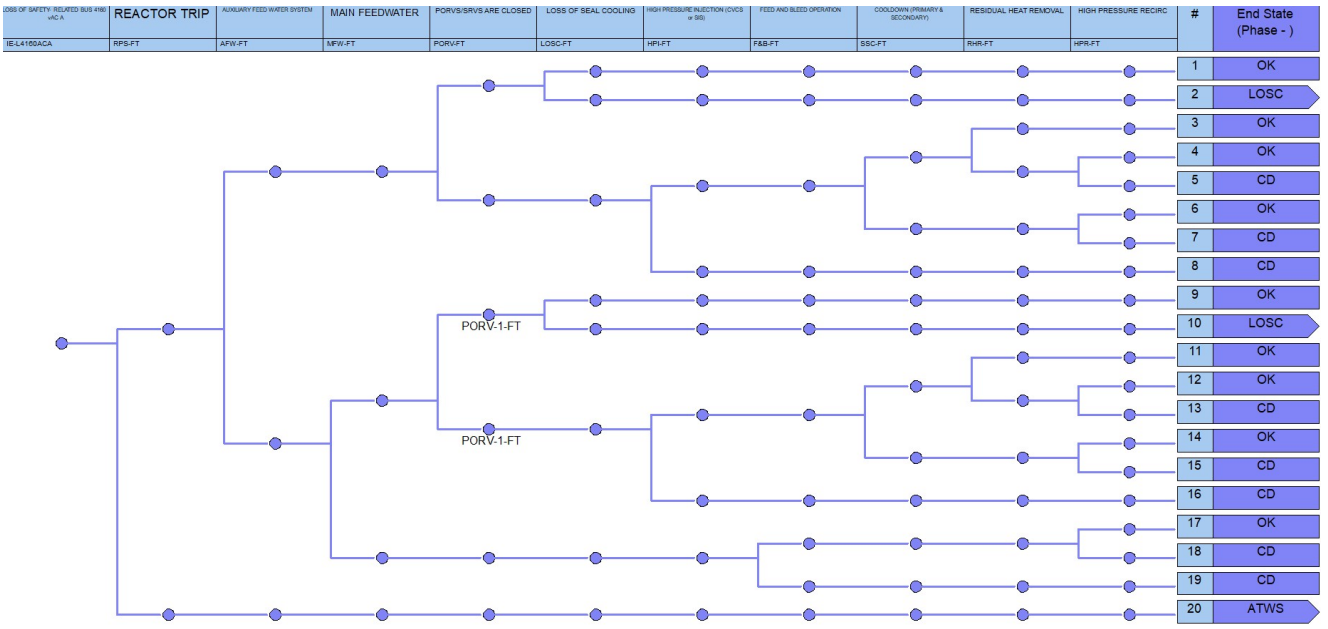

\section{L4160ACB}

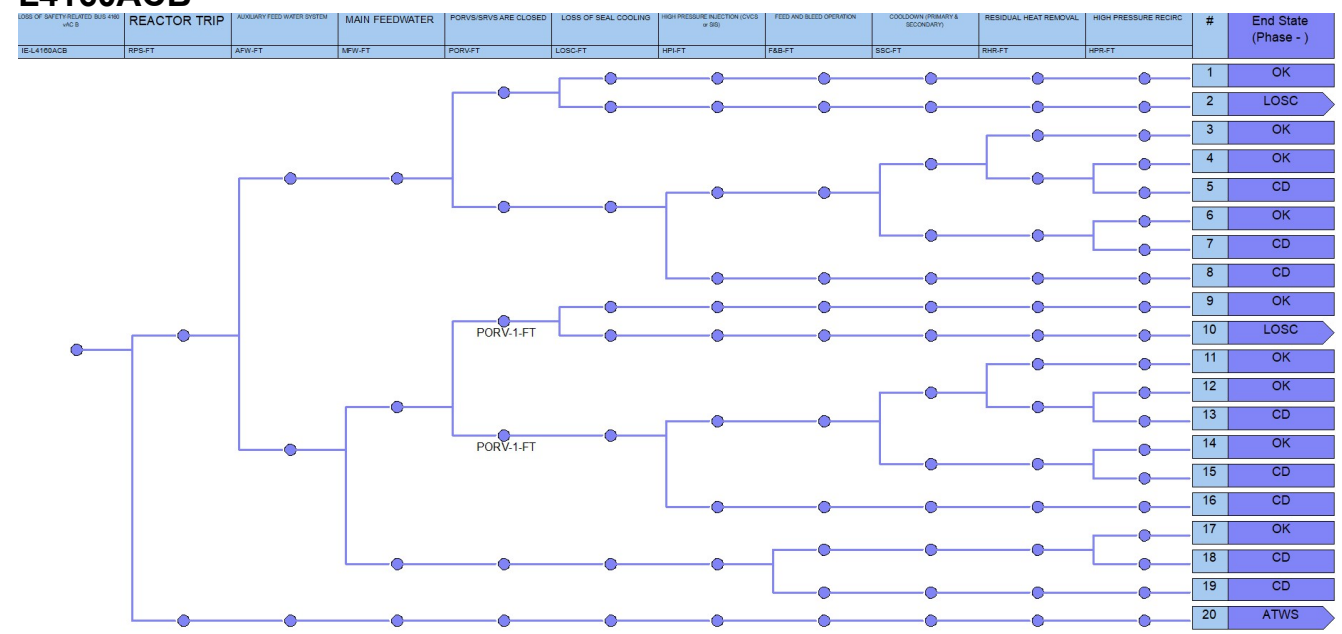

\section{LLOCA}

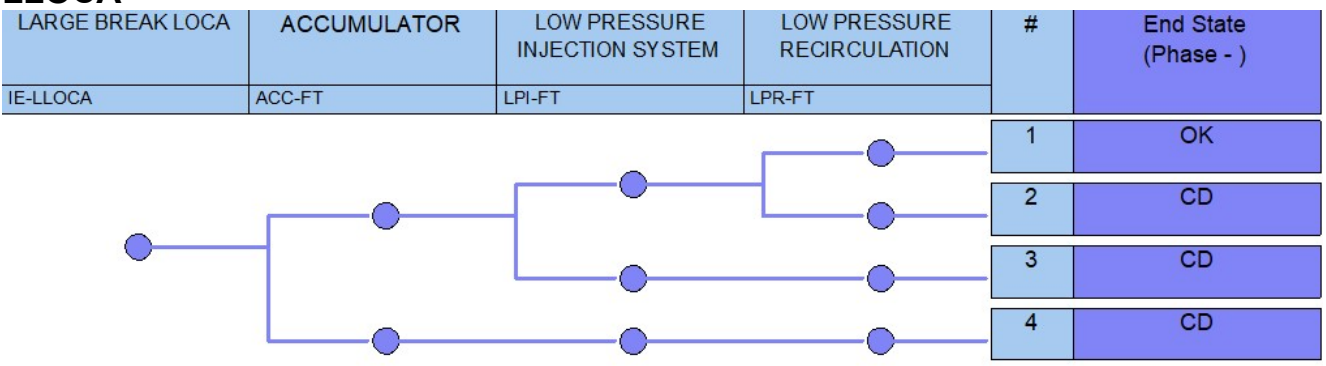

\section{LOCCW}

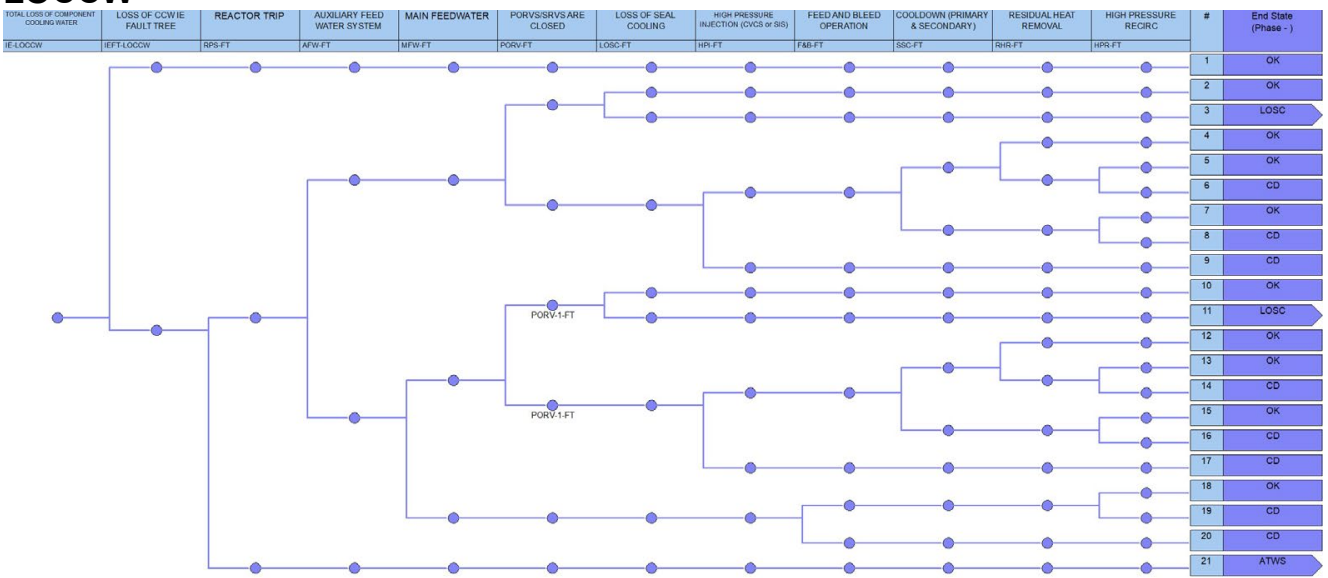

LODCA 


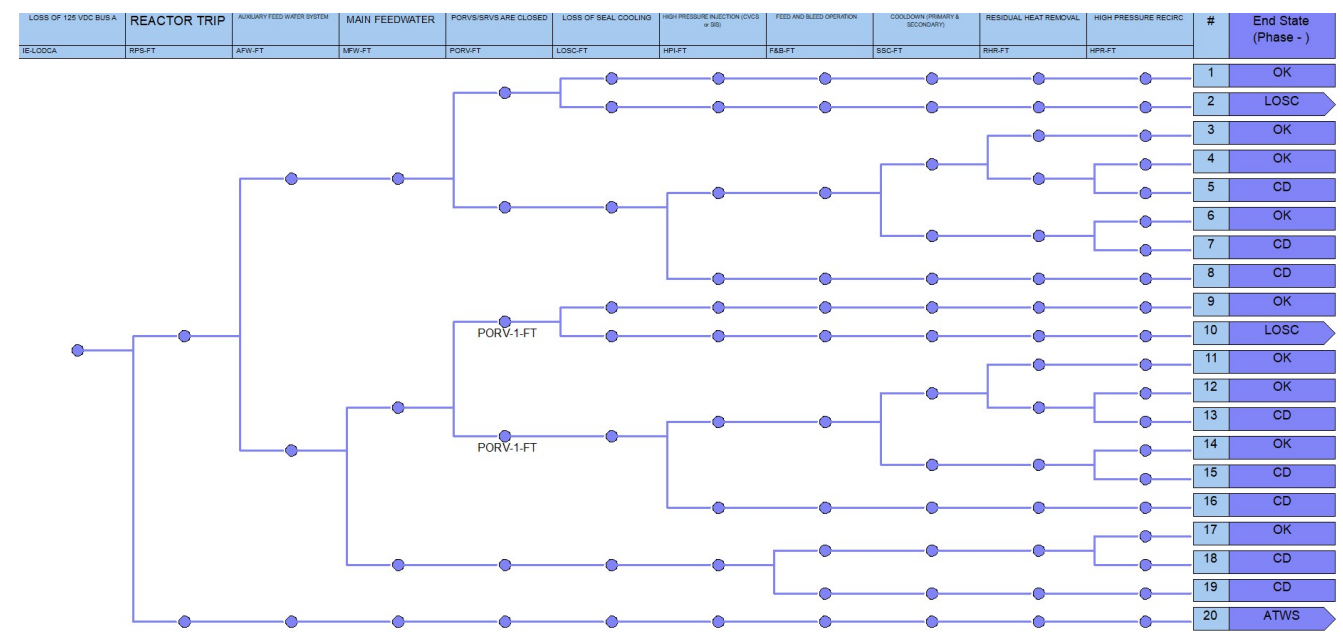

\section{LODCB}

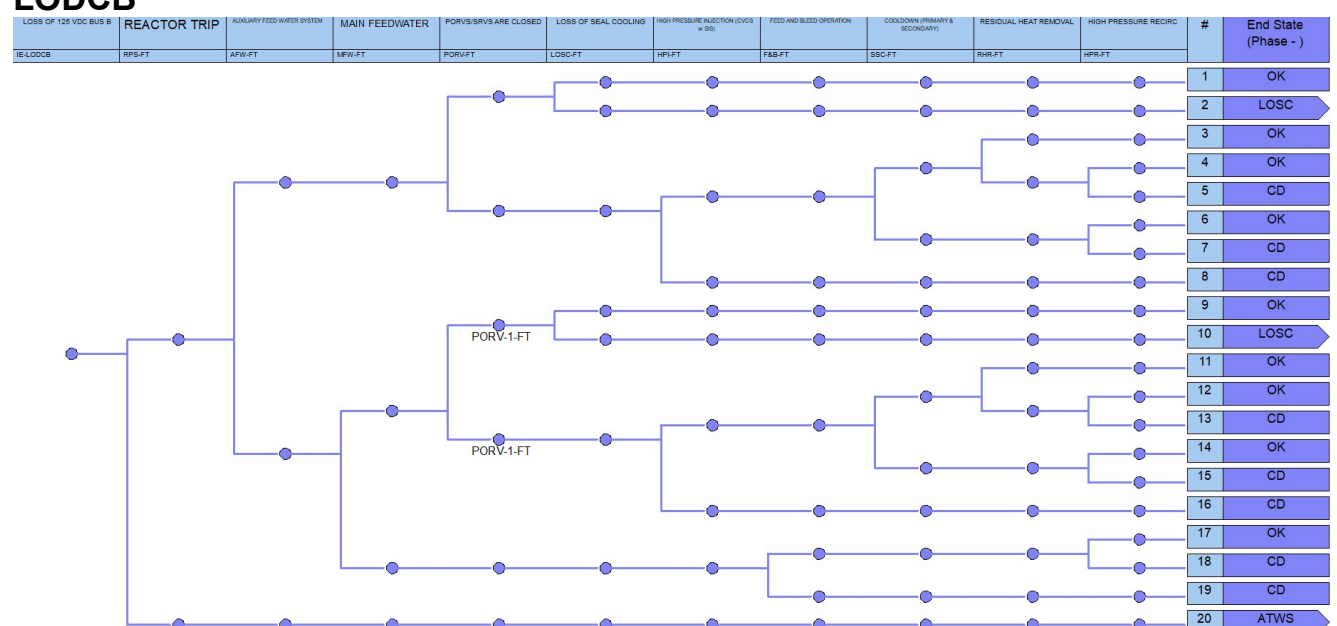

\section{LOMFW}

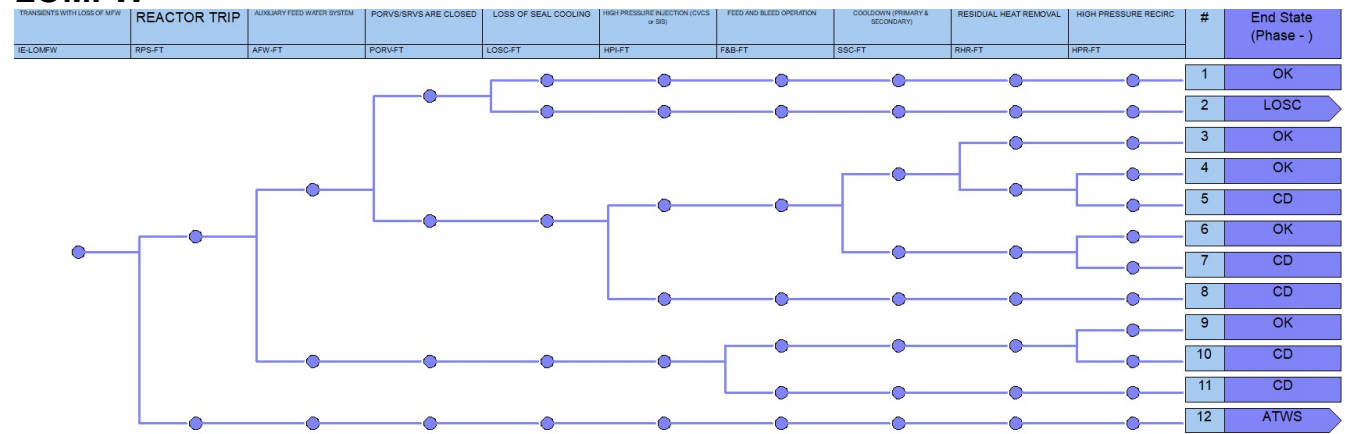

LOOPGR

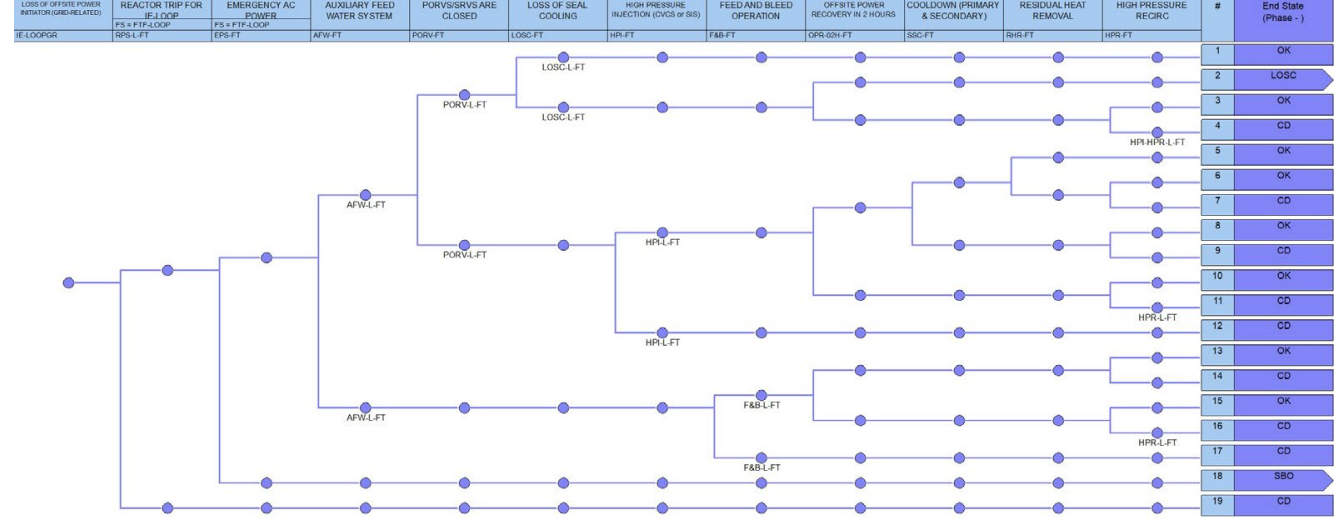




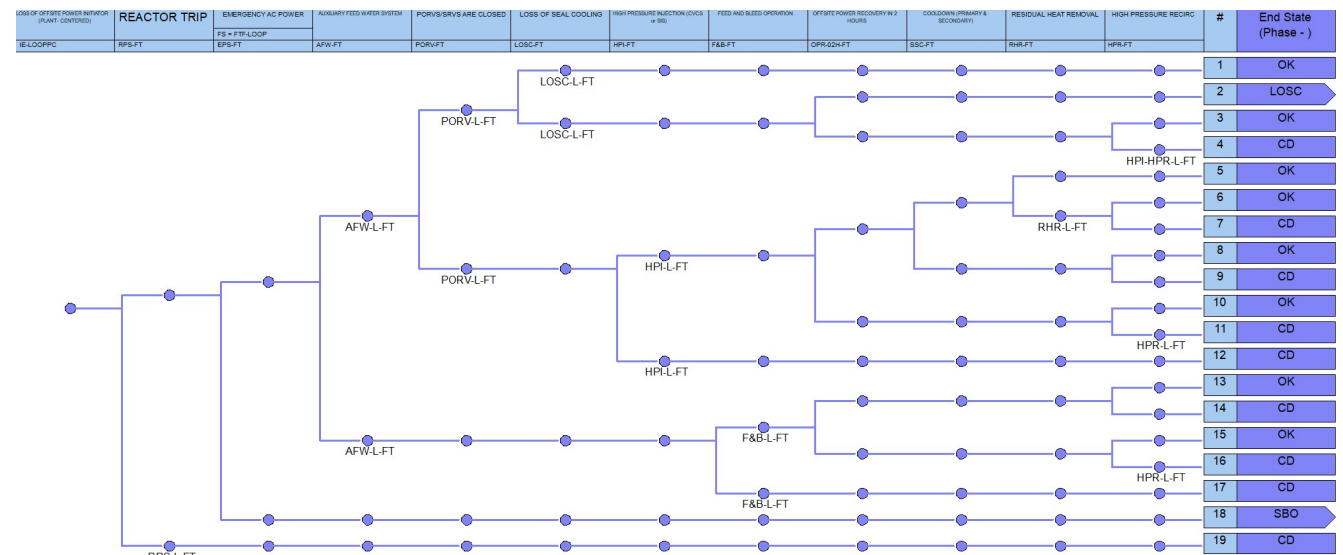

\section{LOOPSC}

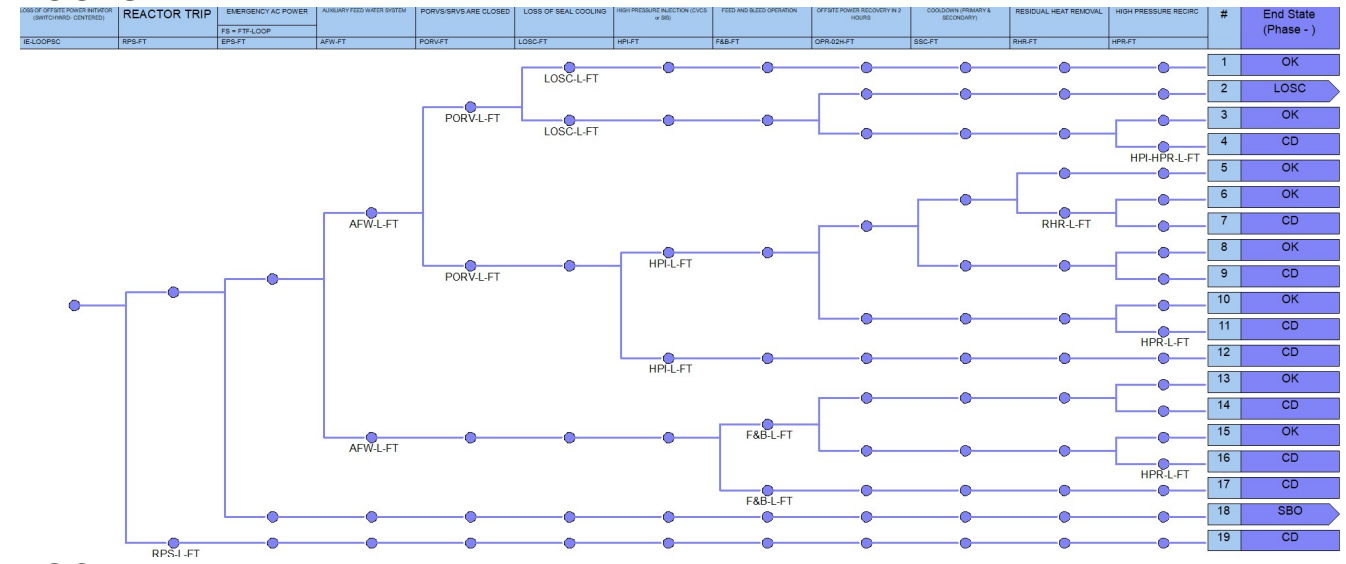

\section{LOOPWR}

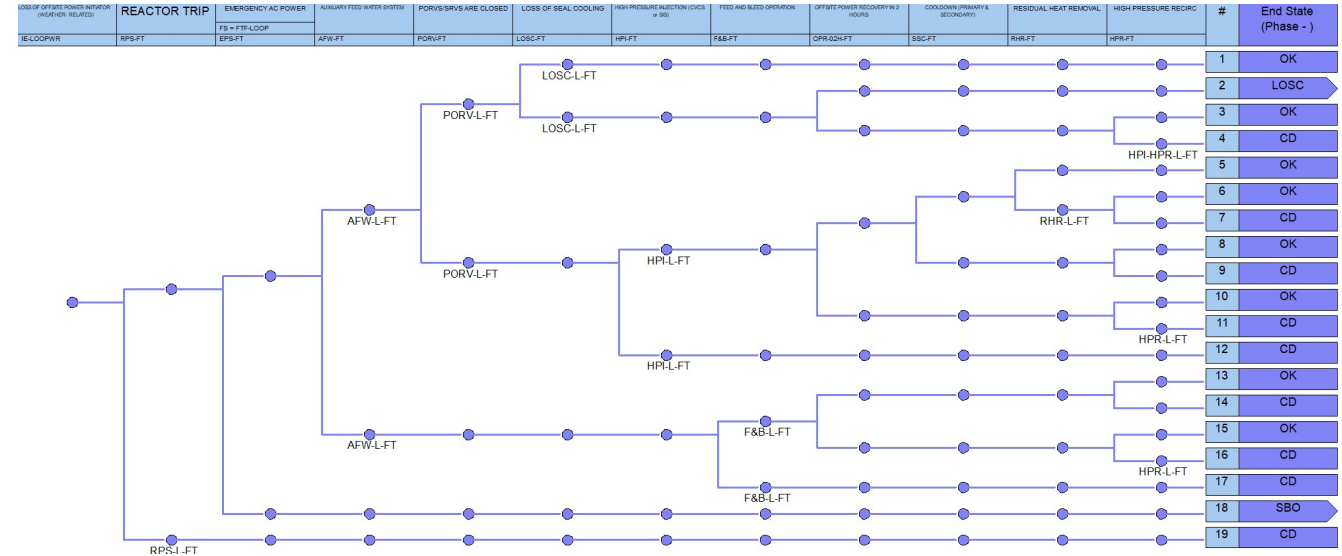

LSSB

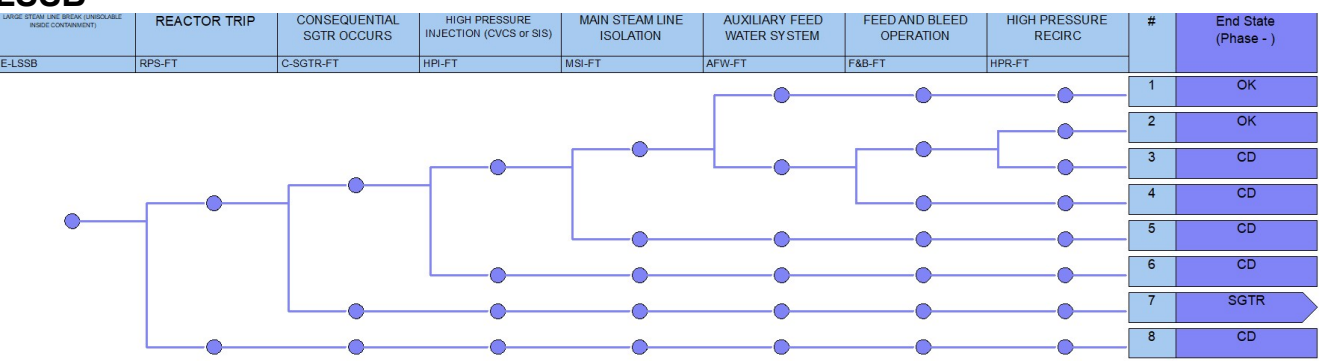

MLOCA 


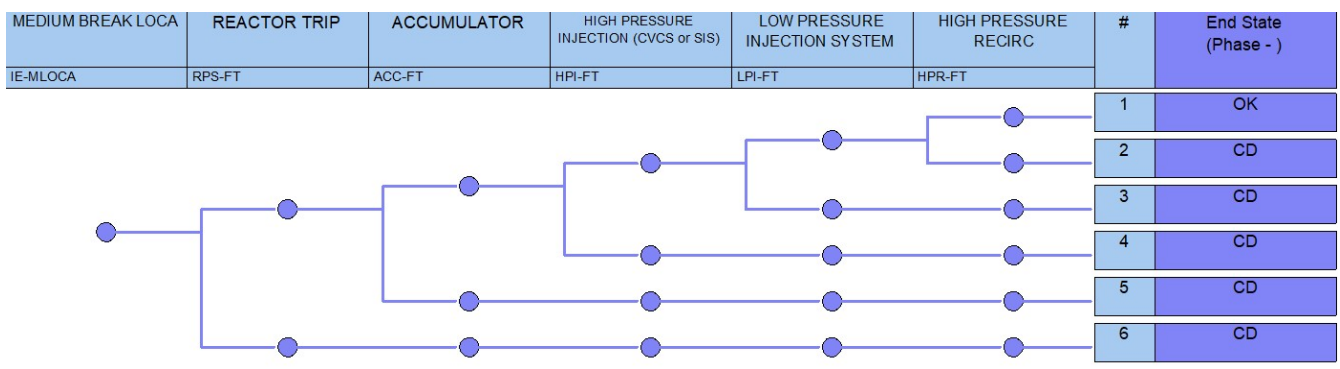

\section{SGTR}

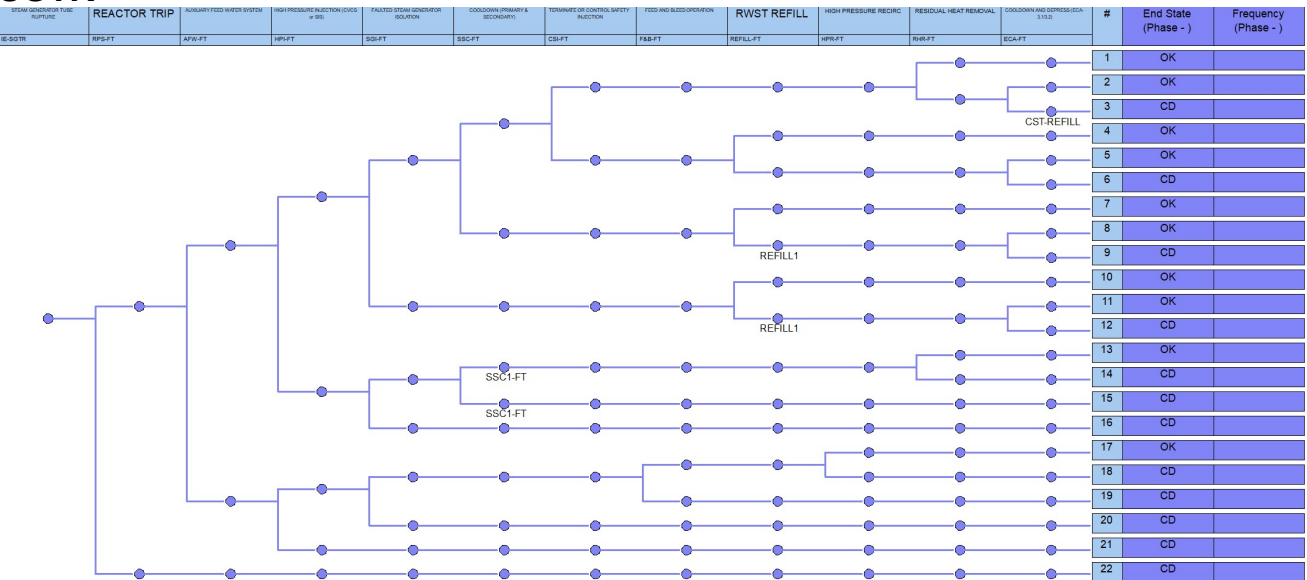

SLOCA

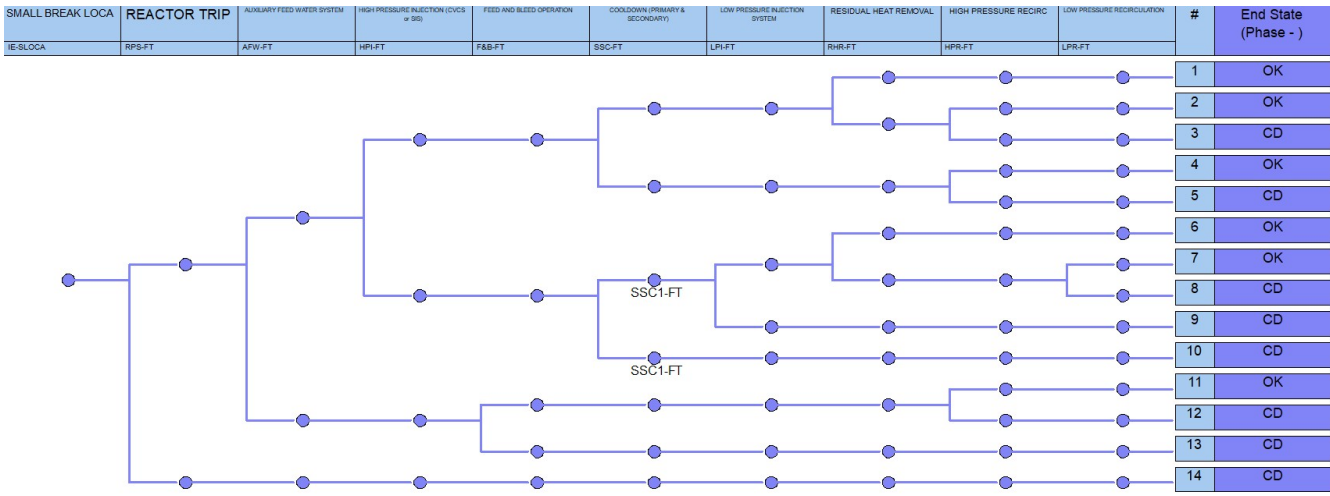

\section{TOR-BIN1}

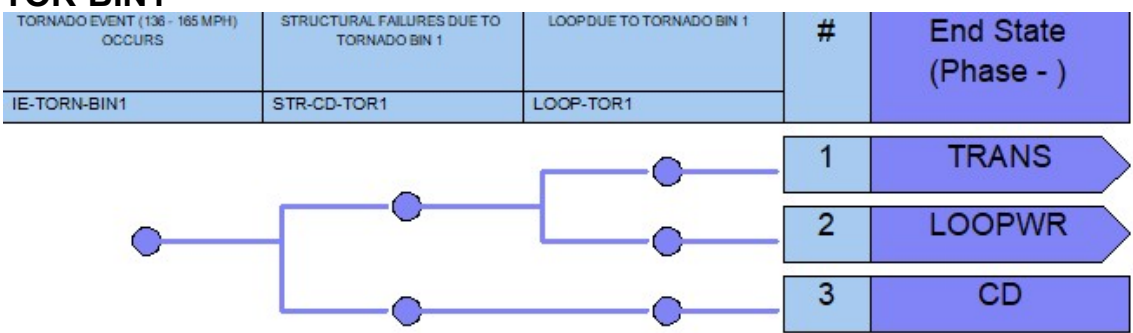

TOR-BIN2

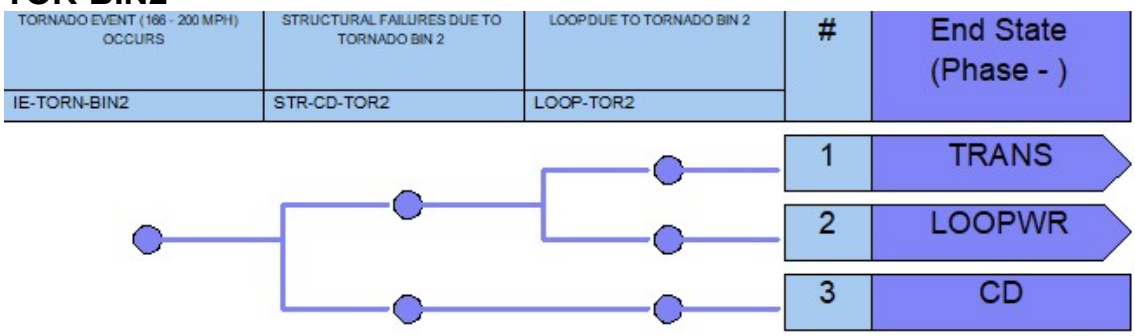

TOR-BIN3 
Generic Pressurized Water Reactor (PWR)

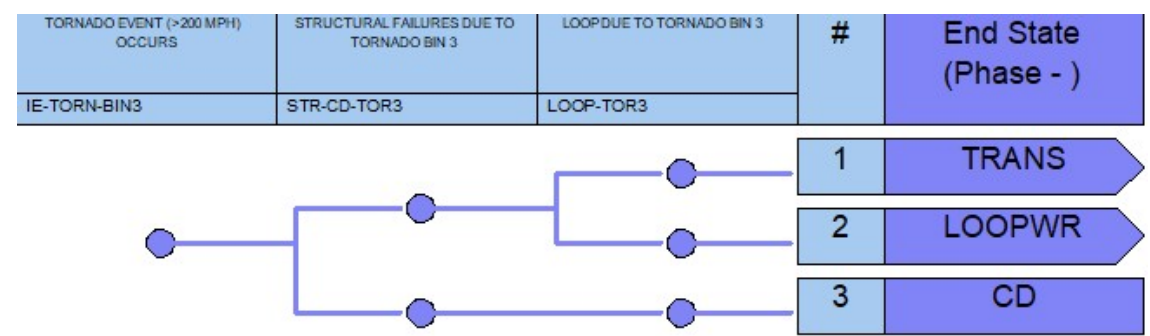

TRANS

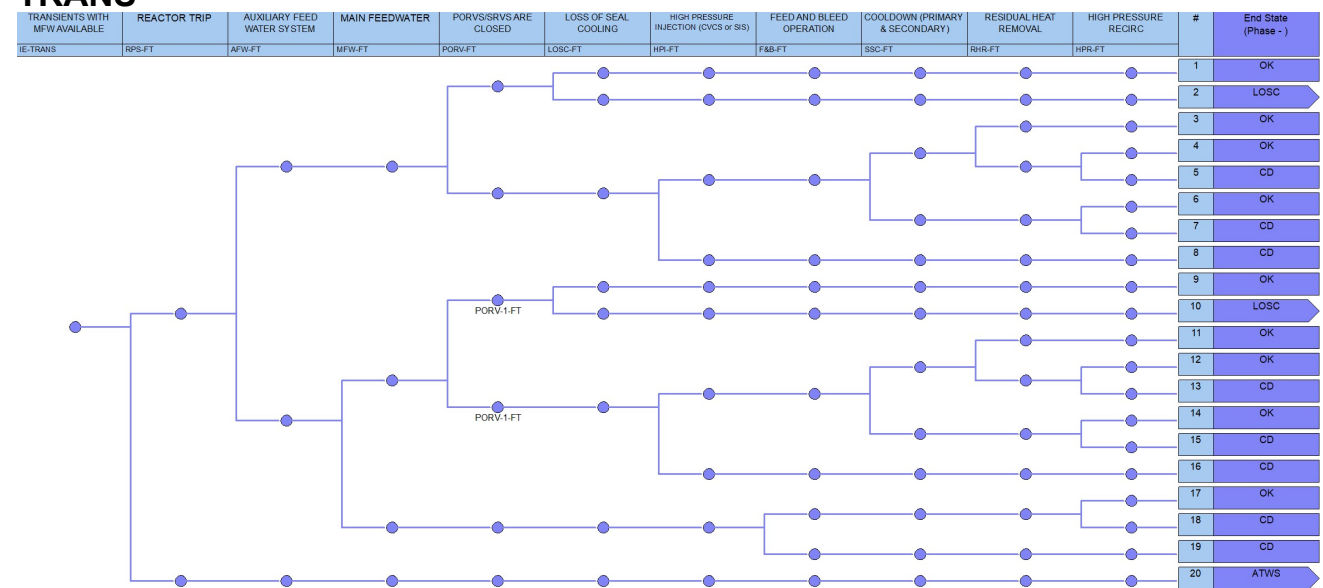

XLOCA

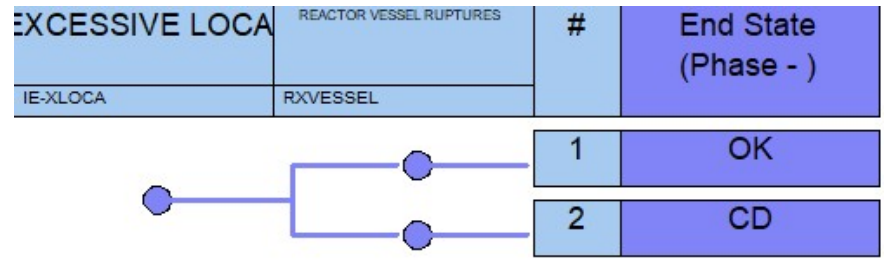




\section{FAULT TREE INFORMATION}




\section{Generic Pressurized Water Reactor (PWR)}

\begin{tabular}{|c|c|}
\hline $\begin{array}{l}\text { Name } \\
\text { A-CDF-ONETOP }\end{array}$ & \\
\hline ACC-FT & \\
\hline AFW-A-FT & \\
\hline AFW-B-FT & \\
\hline AFW-FT & \\
\hline AFW-L-FT & \\
\hline AFW-MAN-TDP & \\
\hline AFW-MDP-EQ-FT & \\
\hline AFW-RECP-FT & \\
\hline ASD-XHE-FT & \\
\hline BORATION-FT & \\
\hline C-SGTR-FT & \\
\hline CD-EQ1-FT & \\
\hline CD-EQ2-FT & \\
\hline CD-EQ3-FT & \\
\hline CD-EQ4-FT & \\
\hline CD-EQ5-FT & \\
\hline CD-EQ6-FT & \\
\hline CD-EQ7-FT & \\
\hline CSI-FT & \\
\hline CST-REFILL & \\
\hline CST-TNK-HWD & \\
\hline CST-TNK-TOR & \\
\hline DGR-01H-FT & \\
\hline DGR-02H-FT & \\
\hline DGR-08H-FT & \\
\hline ECA-FT & \\
\hline ELAP & \\
\hline EPS-FT & \\
\hline EPS-RECP-FT & \\
\hline EVAC & \\
\hline F\&B-FT & \\
\hline F\&B-L-FT & \\
\hline FLEX-480 & \\
\hline FLEX-MUP & \\
\hline FLEX-SGP & \\
\hline FLI-TERM & \\
\hline FRI-SPUR-PORV & \\
\hline HCN-WARN & \\
\hline HPI-FT & \\
\hline HPI-HPR-L-FT & \\
\hline HPI-L-FT & \\
\hline HPI-SIS-FT & \\
\hline HPR-FT & \\
\hline HPR-L-FT & \\
\hline HPR-SIS-FT & \\
\hline IEFT-ISL-RHR-CL-FT & \\
\hline IEFT-ISL-RHR-HLS-FT & \\
\hline IEFT-LOCCW & \\
\hline ISL-DIAG-FT & \\
\hline ISL-RHR-CLI-REC-FT & \\
\hline
\end{tabular}

Model Version: 1.0

\section{Description}

One Top Representation of all ETs ACCUMULATOR AUXILIARY FEEDWATER FOR ATWS AUXILIARY FEEDWATER FOR SBO AUXILIARY FEED WATER SYSTEM AUXILIARY FEED WATER FOR LOOP EVENT Long term manual control of TD AFW pump - no FLEX pump AFW MDP FAILURE FROM SEISMIC EVENT AFW FOR INTERNAL FIRE CONTROL AFW AND PLANT FROM AUX SHUTDOWN PANEL EMERGENCY BORATION CONSEQUENTIAL SGTR OCCURS MAJOR STRUCTURE FAILURE- DIRECT CD FROM SEISMIC EVENT MAJOR STRUCTURE FAILURE- DIRECT CD FROM SEISMIC EVENT BIN 2 MAJOR STRUCTURE FAILURE- DIRECT CD FROM SEISMIC EVENT MAJOR STRUCTURE FAILURE- DIRECT CD FROM SEISMIC EVENT MAJOR STRUCTURE FAILURE- DIRECT CD FROM SEISMIC EVENT MAJOR STRUCTURE FAILURE- DIRECT CD FROM SEISMIC EVENT MAJOR STRUCTURE FAILURE- DIRECT CD FROM SEISMIC EVENT TERMINATE OR CONTROL SAFETY INJECTION OPERATOR FAILS TO REFILL CST CST FAILURE DUE TO HIGH WIND/HURRICANES CST FAILURE DUE TO TORNADO EVENTS OPERATOR FAILS TO RECOVER EMERGENCY DIESEL IN 1 HOUR DIESEL GENERATOR RECOVERY (IN $2 \mathrm{HR}$ ) DIESEL GENERATOR RECOVERY SHORT TERM COOLDOWN AND DEPRESS (ECA- 3.1/3.2)

ELAP is declared when it is needed EMERGENCY AC POWER EMERGENCY AC POWER FOR INTERNAL FIRE MCR IS NOT EVACUATED FEED AND BLEED OPERATION FEED AND BLEED FOR LOOP FLEX diesel is operable and connected to buses Boron injection and RCS makeup with FLEX pump FLEX SG pump is operable FLI TERMINATED SPURIOUSLY OPENING OF PORV PLANT SHUTDOWN DUE TO HURRICANE WARNING HIGH PRESSURE INJECTION (CVCS or SIS) HPI/HPR FUNCTIONS DURING LOOP HPI FOR LOOP SAFETY INJECTION SYSTEM HIGH PRESSURE RECIRC HPR FOR LOOP SAFETY INJECTION SYSTEM DURING RECIRC ISLOCA IN RHR DISCHARGE TO CL ISLOCA FROM RHR SUCTION FROM HL LOSS OF CCW IE FAULT TREE OPERATORS FAIL TO DIAGNOSE ISLOCA OPERATORS RECOVER (ISOLATE) ISLOCA IN RHR CL 


\section{Generic Pressurized Water Reactor (PWR)}

\begin{tabular}{|c|c|}
\hline Name & Description \\
\hline ISL-RHR-HL-REC-FT & OPERATORS RECOVER (ISLOCA RHR HL) \\
\hline ISL-RPT-LPI-FT & LPI PIPE RUPTURE \\
\hline ISL-RPT-RHR-FT & RHR HOT LEG PIPE RUPTURE \\
\hline LLOCA-EQ1-FT & LLOCA DUE TO SEISMIC EVENT - BIN 1 \\
\hline LLOCA-EQ2-FT & LLOCA DUE TO SEISMIC EVENT - BIN 2 \\
\hline LLOCA-EQ3-FT & LLOCA DUE TO SEISMIC EVENT - BIN 3 \\
\hline LLOCA-EQ4-FT & LLOCA DUE TO SEISMIC EVENT - BIN 4 \\
\hline LLOCA-EQ5-FT & LLOCA DUE TO SEISMIC EVENT - BIN 5 \\
\hline LLOCA-EQ6-FT & LLOCA DUE TO SEISMIC EVENT - BIN 6 \\
\hline LOOP-EQ1-FT & LOSS OF OFF-SITE POWER FROM SEISMIC EVENT IN BIN 1 \\
\hline LOOP-EQ2-FT & LOSS OF OFFSITE POWER FROM SEISMIC EVENT IN BIN 2 \\
\hline LOOP-EQ3-FT & LOSS OF OFF-SITE POWER FROM SEISMIC EVENT IN BIN 3 \\
\hline LOOP-EQ4-FT & LOSS OF OFF-SITE POWER FROM SEISMIC EVENT IN BIN 4 \\
\hline LOOP-EQ5-FT & LOSS OF OFF-SITE POWER FROM SEISMIC EVENT IN BIN 5 \\
\hline LOOP-EQ6-FT & LOSS OF OFF-SITE POWER FROM SEISMIC EVENT IN BIN 6 \\
\hline LOOP-HCN1 & LOOP DUE TO HURRICANE BIN 1 \\
\hline LOOP-HCN2 & LOOP DUE TO HURRICANE BIN 2 \\
\hline LOOP-HCN3 & LOOP DUE TO HURRICANE BIN 3 \\
\hline LOOP-HCN4 & LOOP DUE TO HURRICANE BIN 4 \\
\hline LOOP-HWD & LOOP DUE TO 136 MPH HIGH WIND EVENT \\
\hline LOOP-TOR1 & LOOP DUE TO TORNADO BIN 1 \\
\hline LOOP-TOR2 & LOOP DUE TO TORNADO BIN 2 \\
\hline LOOP-TOR3 & LOOP DUE TO TORNADO BIN 3 \\
\hline LOSC-FT & LOSS OF SEAL COOLING \\
\hline LOSC-L-FT & LOSC FOR LOOP \\
\hline LPI-FT & LOW PRESSURE INJECTION SYSTEM \\
\hline LPR-FT & LOW PRESSURE RECIRCULATION \\
\hline MFW-FT & MAIN FEEDWATER \\
\hline MSI-FT & MAIN STEAM LINE ISOLATION \\
\hline MUT-EX & MUTUALLY EXCLUSIVE \\
\hline OPR-01H-FT & OFFSITE POWER RECOVERY IN 1 HOUR \\
\hline OPR-02H-FT & OFFSITE POWER RECOVERY IN 2 HOURS \\
\hline OPR-08H-FT & OFFSITE POWER RECOVERY IN 8 HOURS \\
\hline OPR-24HR & AC power recovery in 24 hours given failure at battery depletion \\
\hline OPR-72HR & AC power Recovery in 72 hours given failure at battery depletion \\
\hline PORV-1-FT & PORV WHEN AFW FAILED \\
\hline PORV-A-FT & PORVS ARE CLOSED (ATWS) \\
\hline PORV-B-FT & PORVS/SRVS OPEN DURING STATION BLACKOUT \\
\hline PORV-FT & PORVS/SRVS ARE CLOSED \\
\hline PORV-L-FT & PORV FOR LOOP \\
\hline PZR-FT & RCS DEPRESSURIZATION FOR LPI/RHR \\
\hline RCPSLOCA-FT & RCP SEAL LOCA \\
\hline RCPT-FT & REACTOR COOLANT PUMPS TRIPPED \\
\hline RCSPRESS-FT & LIMITING RCS PRESSURE \\
\hline REFILL-FT & RWST REFILL \\
\hline REFILL1 & RWST REFILL \\
\hline RHR-FT & RESIDUAL HEAT REMOVAL \\
\hline RHR-L-FT & RESIDUAL HEAT REMOVAL FOR LOOP \\
\hline RPS-FT & REACTOR TRIP \\
\hline RPS-L-FT & REACTOR TRIP FOR IE-LOOP \\
\hline RSD-B-FT & RAPID SECONDARY DEPRESS \\
\hline RSD-FT & RAPID SECONDARY DEPRESSURIZATION (<1710 PSI IN 2 HR) \\
\hline RWST-TNK-HWD & RWST FAILURE DUE TO HIGH WIND/HURRICANES \\
\hline
\end{tabular}




\section{Generic Pressurized Water Reactor (PWR)}

\begin{tabular}{|c|c|}
\hline Name & Description \\
\hline RWST-TNK-TOR & RWST FAILURE DUE TO TORNADO EVENTS \\
\hline RXVESSEL & REACTOR VESSEL RUPTURES \\
\hline SGI-FT & FAULTED STEAM GENERATOR ISOLATION \\
\hline SLOCA-EQ1-FT & SMALL LOCA DUE TO SEISMIC EVENT - BIN 1 \\
\hline SLOCA-EQ2-FT & SMALL LOCA DUE TO SEISMIC EVENT - BIN 2 \\
\hline SLOCA-EQ3-FT & SMALL LOCA DUE TO SEISMIC EVENT - BIN 3 \\
\hline SLOCA-EQ4-FT & SMALL LOCA DUE TO SEISMIC EVENT - BIN 4 \\
\hline SLOCA-EQ5-FT & SMALL LOCA DUE TO SEISMIC EVENT - BIN 5 \\
\hline SLOCA-EQ6-FT & SMALL LOCA DUE TO SEISMIC EVENT - BIN 6 \\
\hline SSC-FT & COOLDOWN (PRIMARY \& SECONDARY) \\
\hline SSC1-FT & COOLDOWN (PRIMARY \& SECONDARY) \\
\hline STR-CD-HCN1 & STRUCTURAL FAILURES DUE TO HURRICANE BIN 1 \\
\hline STR-CD-HCN2 & STRUCTURAL FAILURES DUE TO HURRICANE BIN 2 \\
\hline STR-CD-HCN3 & STRUCTURAL FAILURES DUE TO HURRICANE BIN 3 \\
\hline STR-CD-HCN4 & STRUCTURAL FAILURES DUE TO HURRICANE BIN 4 \\
\hline STR-CD-HWD & STRUCTURAL FAILURES DUE TO HIGH WINDS \\
\hline STR-CD-TOR1 & STRUCTURAL FAILURES DUE TO TORNADO BIN 1 \\
\hline STR-CD-TOR2 & STRUCTURAL FAILURES DUE TO TORNADO BIN 2 \\
\hline STR-CD-TOR3 & STRUCTURAL FAILURES DUE TO TORNADO BIN 3 \\
\hline SWS-FT & SERVICE WATER SYSTEM \\
\hline SWS-STR-HWD & SWS STRAINER FAILURE DUE TO HIGH WIND/HURRICANES \\
\hline SWS-STR-TOR & SWS STRAINER FAILURE DUE TO TORNADOS \\
\hline Z-EQK-SEQ-4-03 & SUCCESS EVENTS FOR SEQUENCE 4-03 \\
\hline Z-EQK-SEQ-4-05 & SUCCESS EVENTS FOR SEQUENCE 4-05 \\
\hline Z-EQK-SEQ-4-08 & SUCCESS EVENTS FOR SEQUENCE 4-08 \\
\hline Z-EQK-SEQ-4-09 & SUCCESS EVENTS FOR SEQUENCE 4-09 \\
\hline Z-EQK-SEQ-4-10 & SUCCESS EVENTS FOR SEQUENCE 4-10 \\
\hline Z-EQK-SEQ-4-12 & SUCCESS EVENTS FOR SEQUENCE 4-12 \\
\hline Z-EQK-SEQ-4-13 & SUCCESS EVENTS FOR SEQUENCE 4-13 \\
\hline Z-EQK-SEQ-4-14 & SUCCESS EVENTS FOR SEQUENCE 4-14 \\
\hline Z-EQK-SEQ-5-2 & SUCCESS EVENTS FOR SEQUENCE 5-2 \\
\hline Z-EQK-SEQ-5-3 & SUCCESS EVENTS FOR SEQUENCE 5-3 \\
\hline Z-EQK-SEQ-5-4 & SUCCESS EVENTS FOR SEQUENCE 5-4 \\
\hline Z-EQK-SEQ-6-2 & SUCCESS EVENTS FOR SEQUENCE 6-2 \\
\hline Z-EQK-SEQ-6-3 & SUCCESS EVENTS FOR SEQUENCE 6-3 \\
\hline Z-EQK-SEQ-6-4 & SUCCESS EVENTS FOR SEQUENCE 6-4 \\
\hline ZV-TOP & DUMMY TOP \\
\hline
\end{tabular}




\section{ACC-FT}

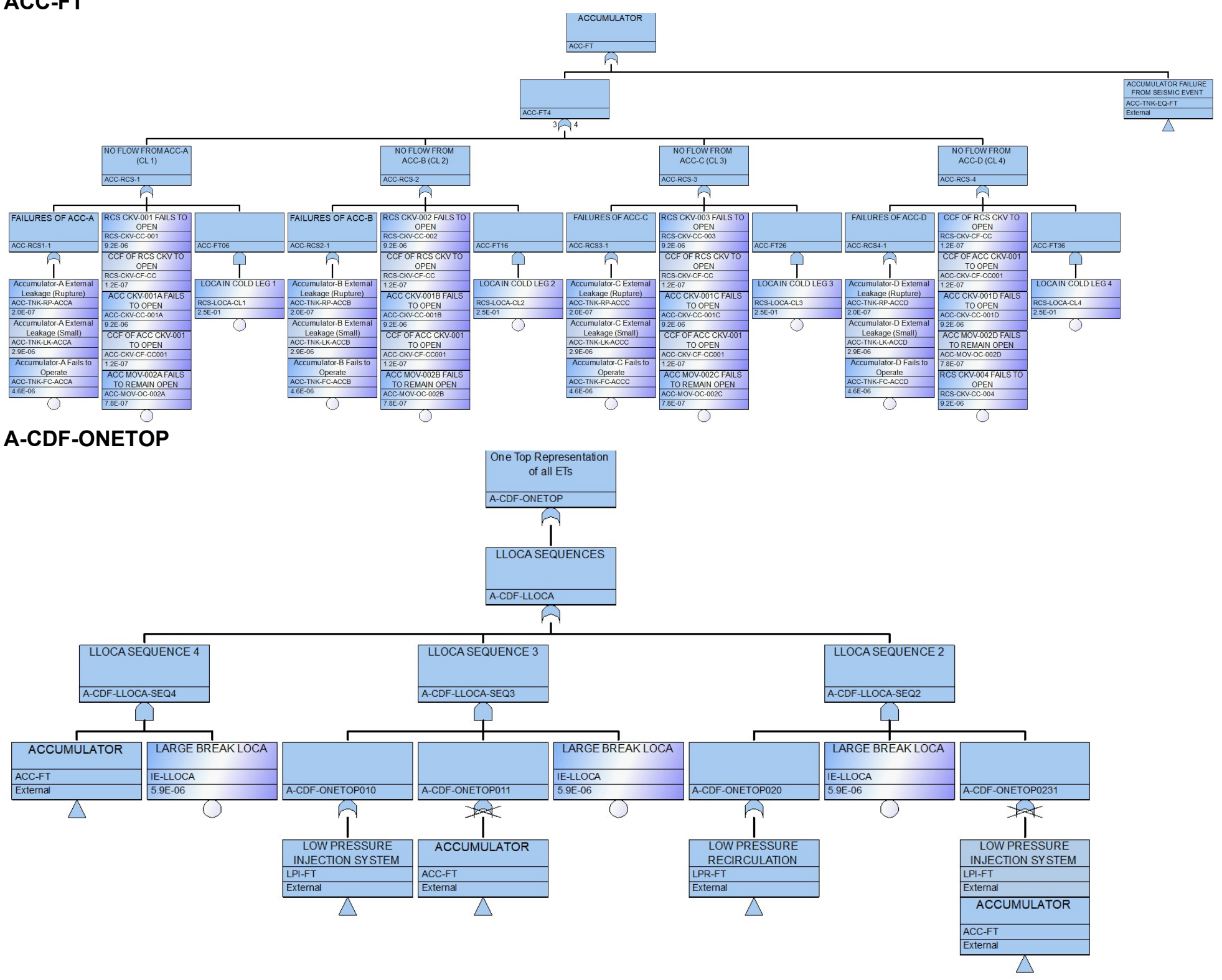

\section{AFW-A-FT}




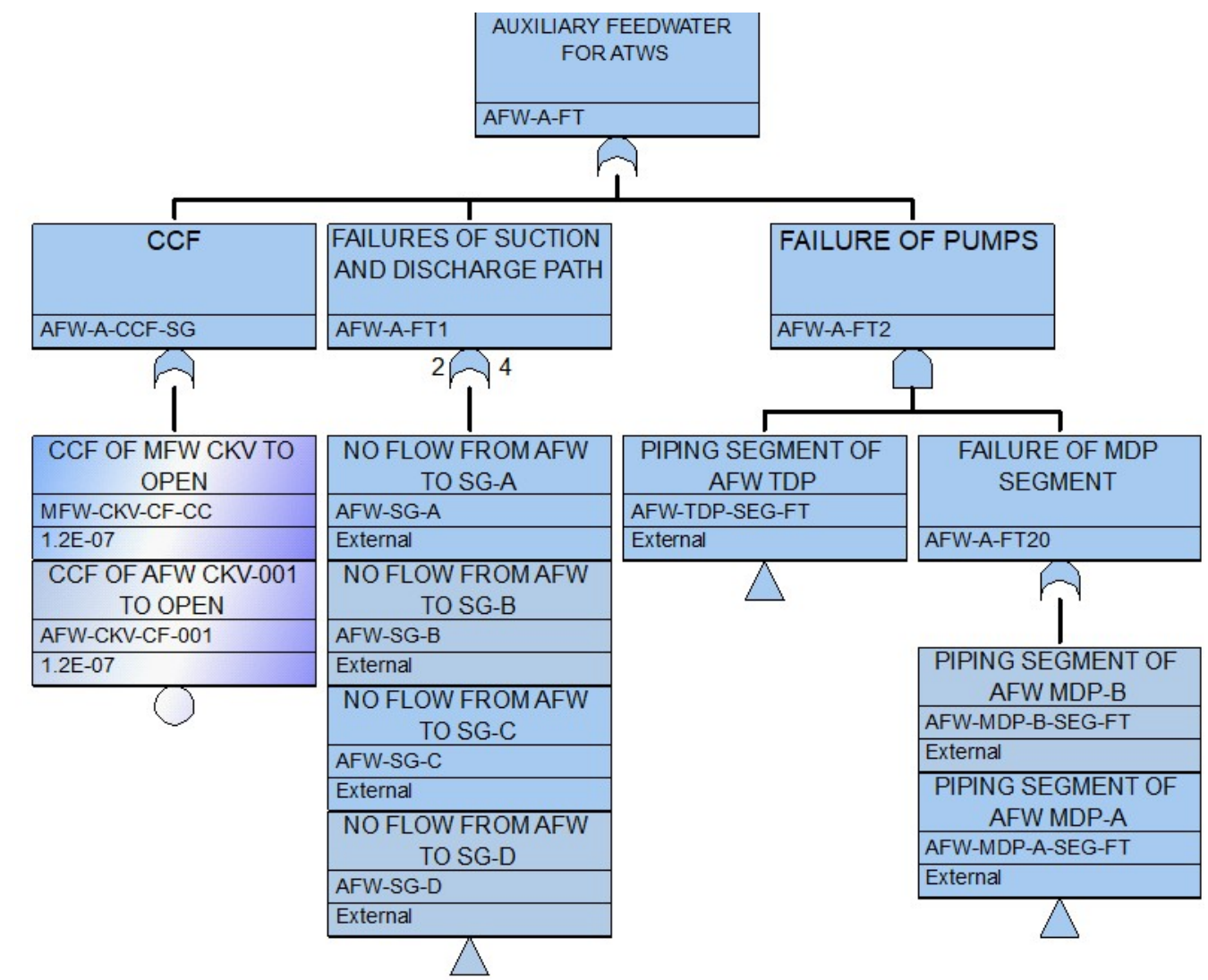

\section{AFW-B-FT} AUXILIARY FEEDWATER FOR SBO AFW-B-FT

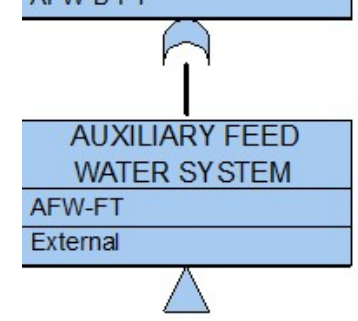

AFW-FT 
Generic Pressurized Water Reactor (PWR)

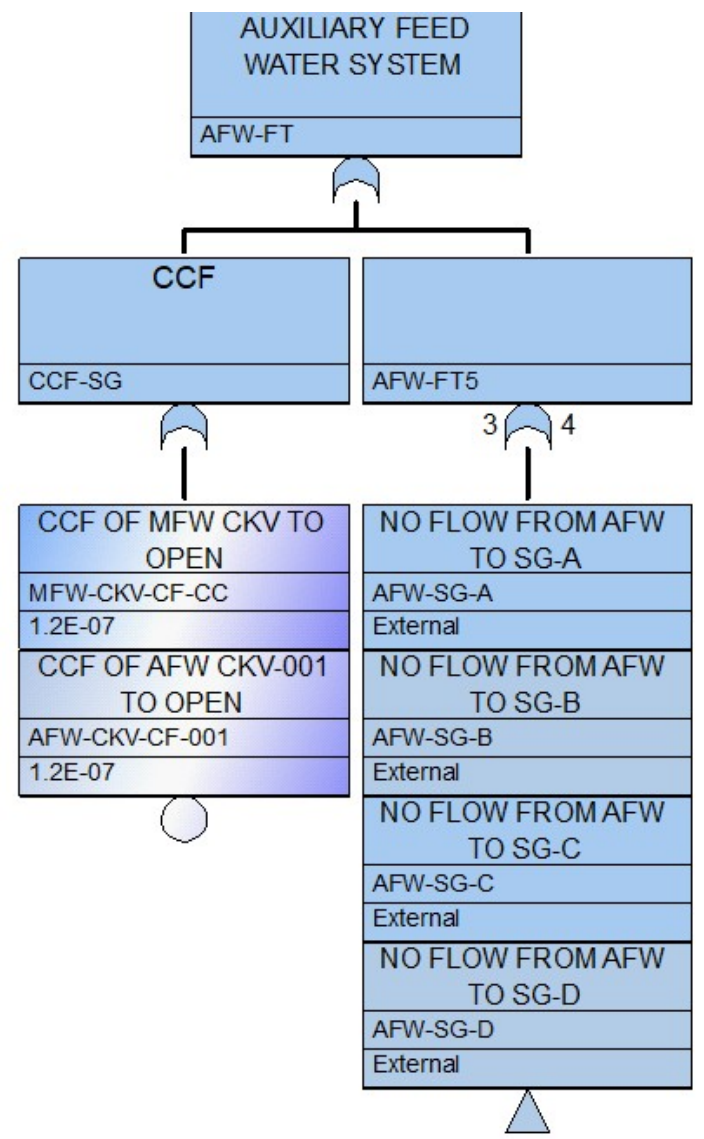

AFW-L-FT

AUXILIARY FEED WATER FOR LOOP EVENT

AFW-L-FT

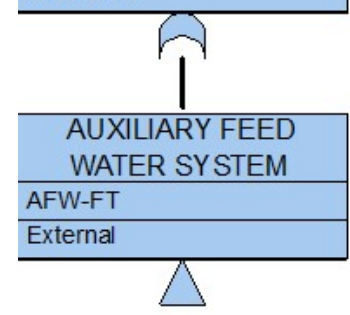

\section{AFW-MAN-TDP}

Long term manual control of TD

AFW pump - no FLEX pump

AFW-MAN-TDP

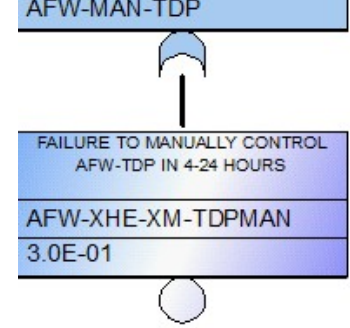

AFW-MDP-EQ-FT 


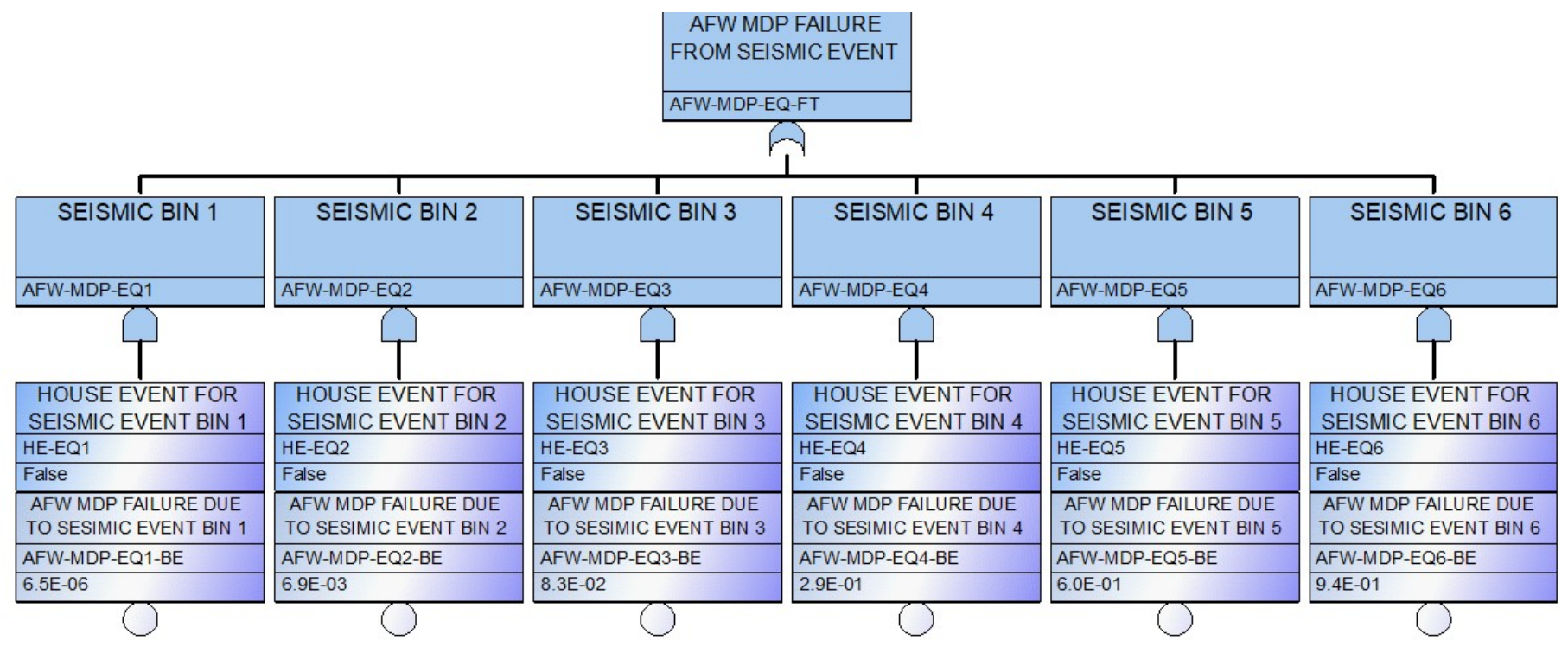

\section{AFW-RECP-FT}

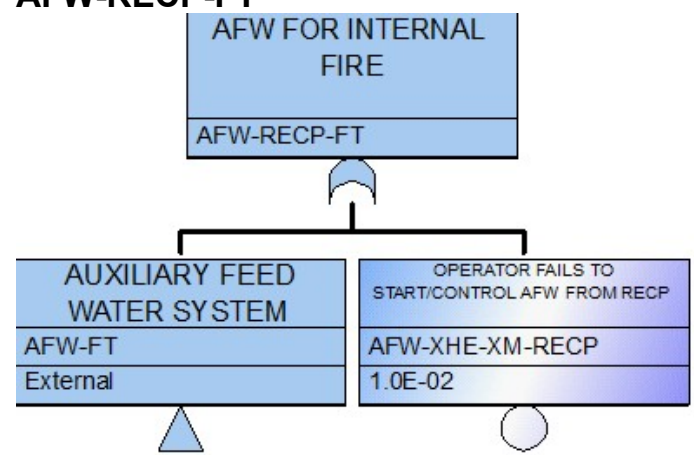

\section{ASD-XHE-FT}

CONTROLAFW AND PLANT FROM AUX SHUTDOWN PANEL

ASD-XHE-FT

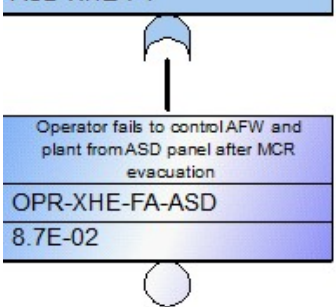

\section{BORATION-FT}

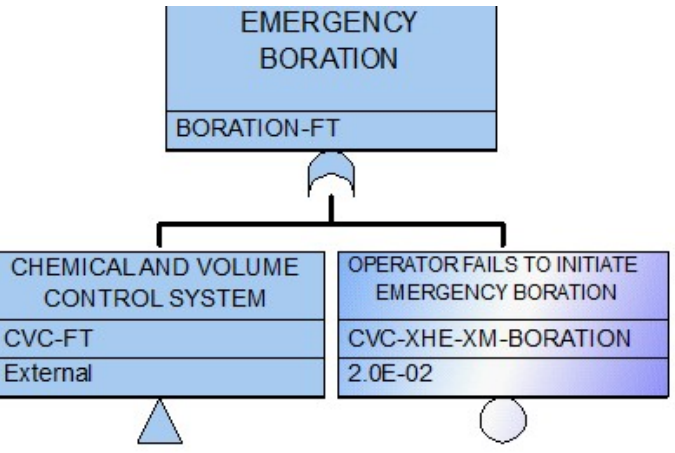

\section{CD-EQ1-FT}


Generic Pressurized Water Reactor (PWR)

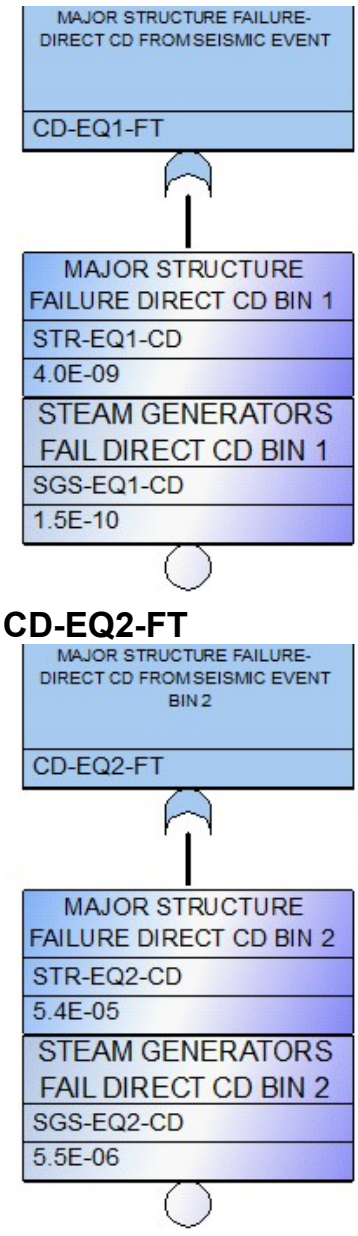

\section{CD-EQ3-FT}

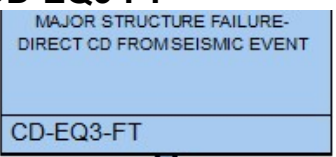

CD-EQ3-FT

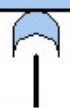

MAJOR STRUCTURE FAILURE DIRECT CD BIN 3 STR-EQ3-CD

2.6E-03

STEAM GENERATORS

FAIL DIRECT CD BIN 3

SGS-EQ3-CD

4.5E-04

\section{CD-EQ4-FT}


Generic Pressurized Water Reactor (PWR)

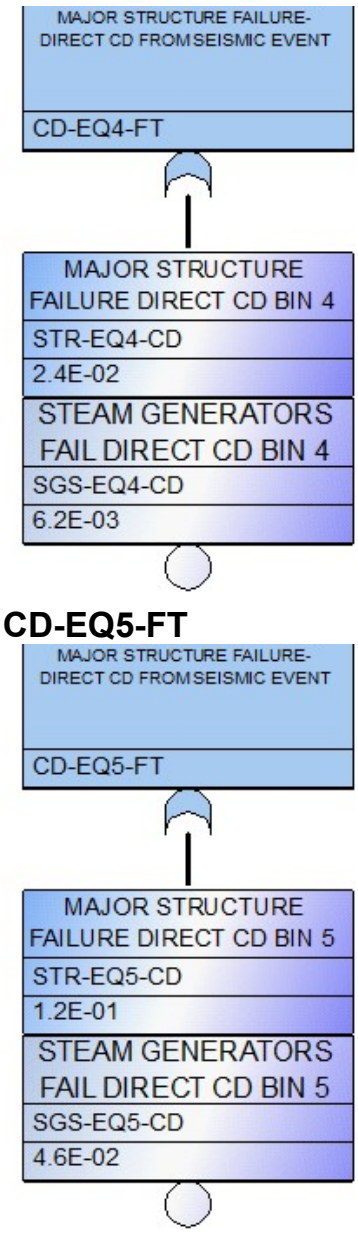

\section{CD-EQ6-FT}

\begin{tabular}{|l|} 
MAANOR STRUCTURE FAILURE- \\
DIRECT CD FROM SEISMIC EVENT \\
\hline CD-EQ6-FT \\
\hline
\end{tabular}

F

MAJOR STRUCTURE FAILURE DIRECT CD BIN 6

STR-EQ6-CD

5.6E-01

STEAM GENERATORS

FAIL DIRECT CD BIN 6

SGS-EQ6-CD

3.5E-01

\section{CD-EQ7-FT}


Generic Pressurized Water Reactor (PWR)

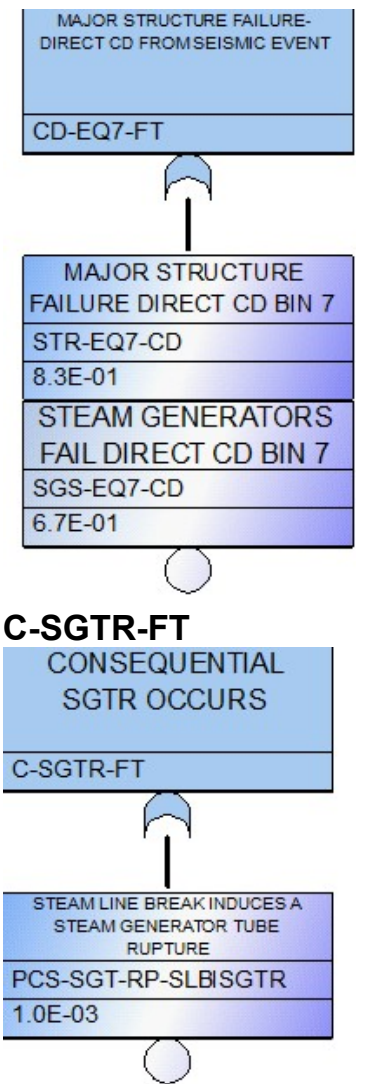

\section{CSI-FT}

TERMINATE OR CONTROL SAFETY INJECTION

CSI-FT

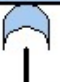

OPERATOR FAILS TO CONTROL/TERMINATE SI HPI-XHE-XM-THRTL $1.0 \mathrm{E}-03$

\section{CST-REFILL}

\begin{tabular}{l} 
OPERATOR FAILS TO \\
REFILL CST \\
\hline CST-REFILL \\
\hline
\end{tabular}

\begin{tabular}{l} 
OPERATOR FAILS TO \\
REFILL CST \\
\hline \begin{tabular}{l} 
AFW-XHE-XM-CST \\
$1.0 \mathrm{E}-04$ \\
\hline
\end{tabular}
\end{tabular}

\section{CST-TNK-HWD}




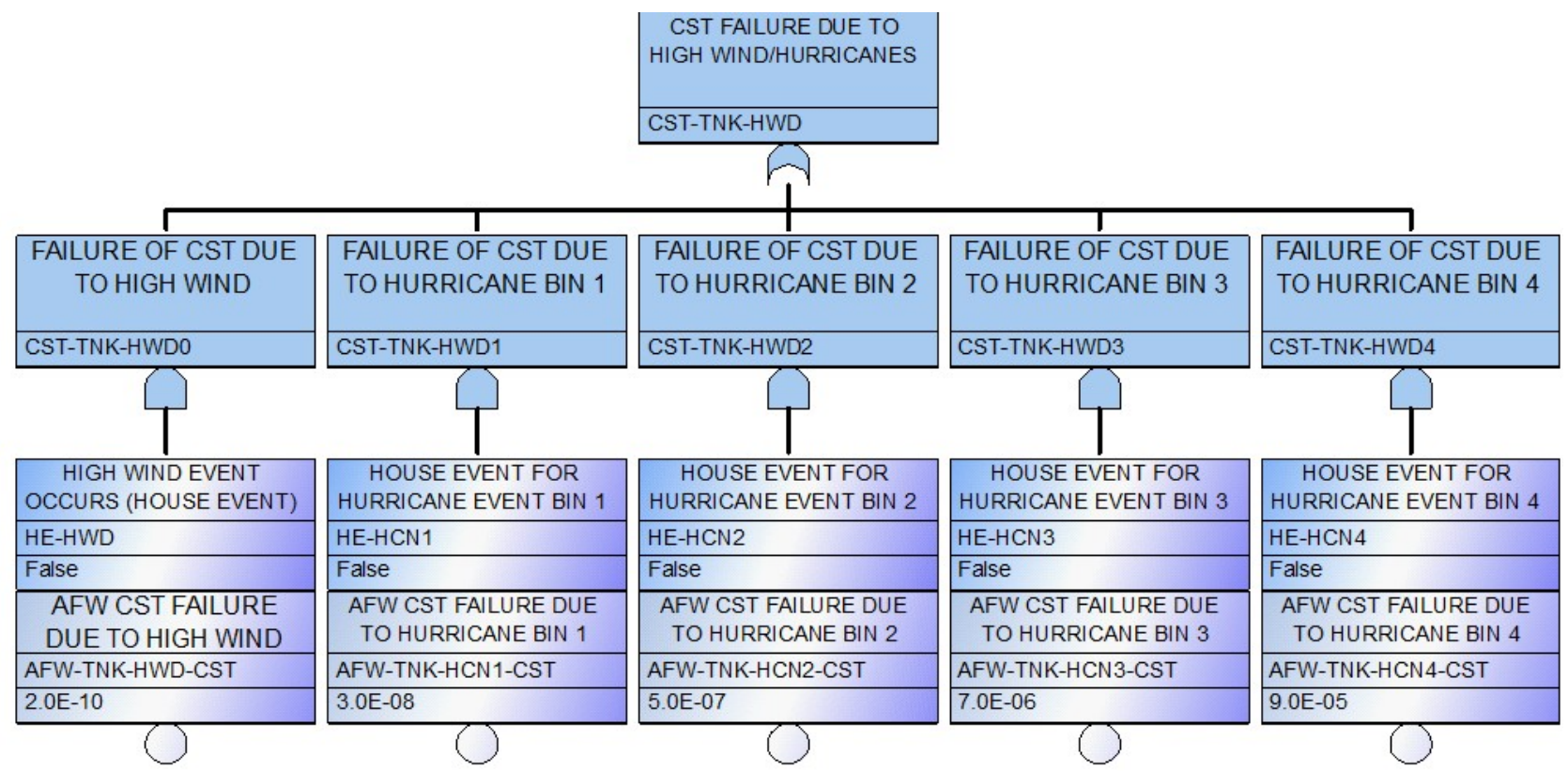

\section{CST-TNK-TOR}

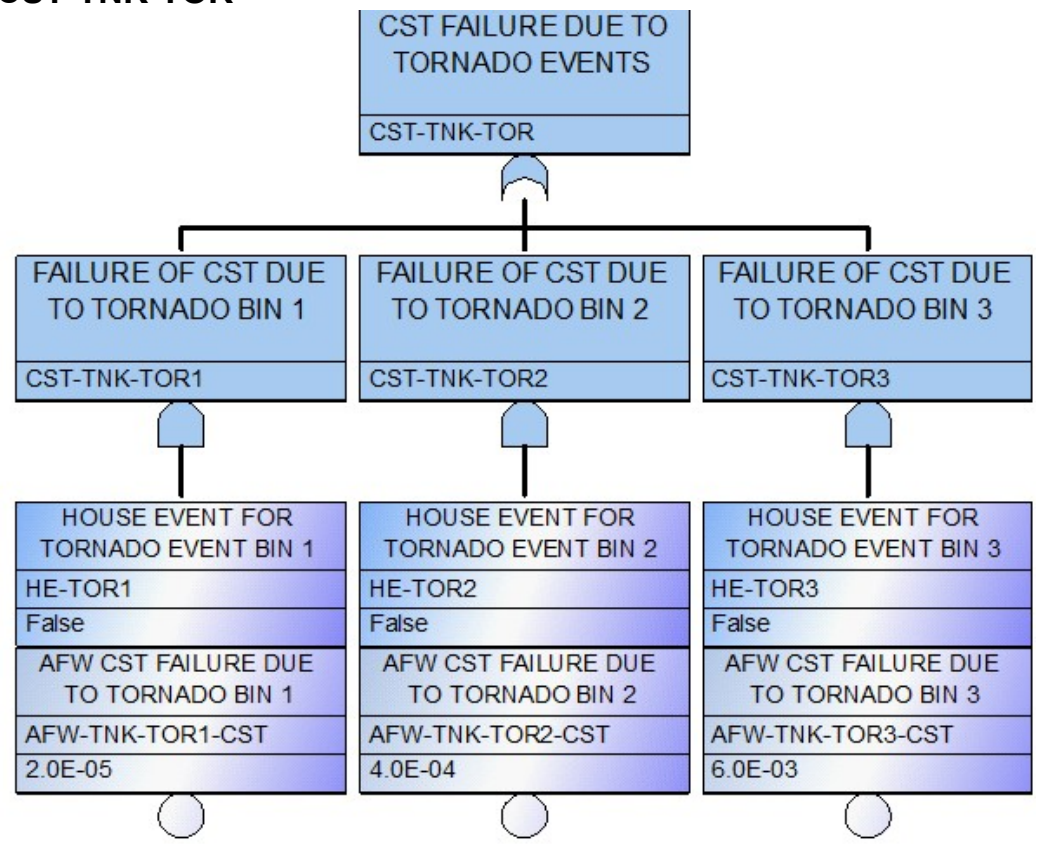

\section{DGR-01H-FT}

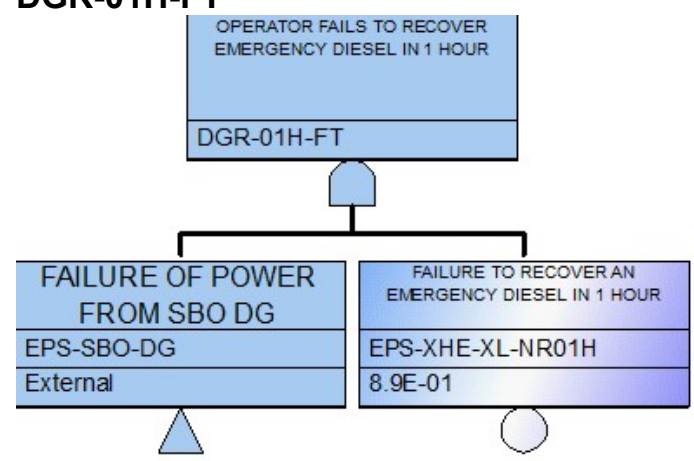

DGR-02H-FT 
Generic Pressurized Water Reactor (PWR)

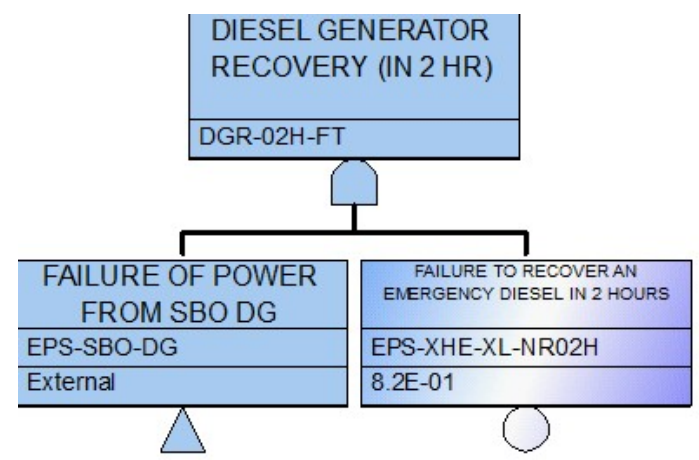

\section{DGR-08H-FT}

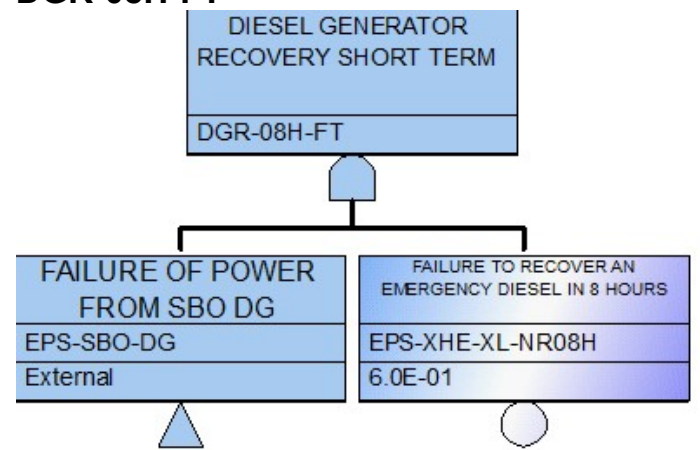

\section{ECA-FT}

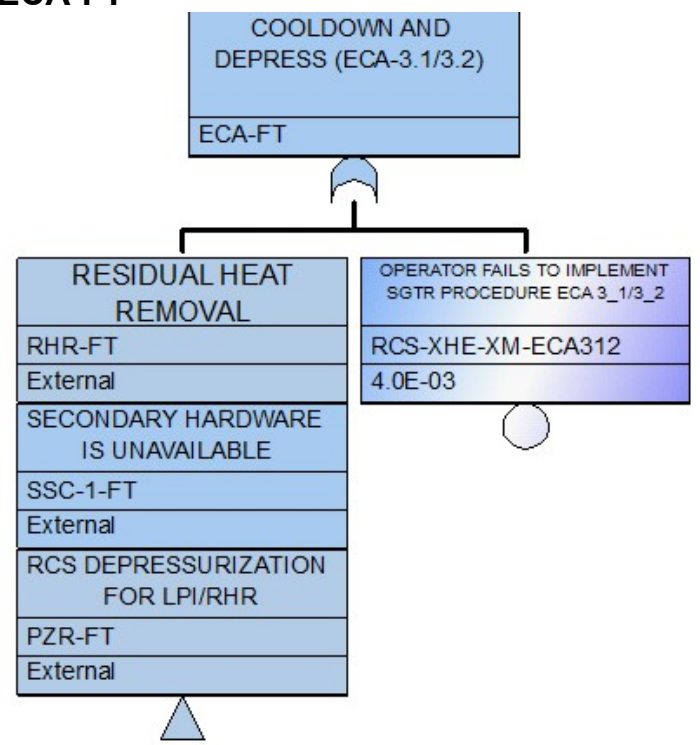

\section{ELAP}

ELAP is declared when it is needed

ELAP

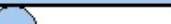

1

Operators fail to declare

ELAP when ben eficial FLX-XHE-XE-ELAP

1.0E-02

EPS-FT 
Generic Pressurized Water Reactor (PWR)

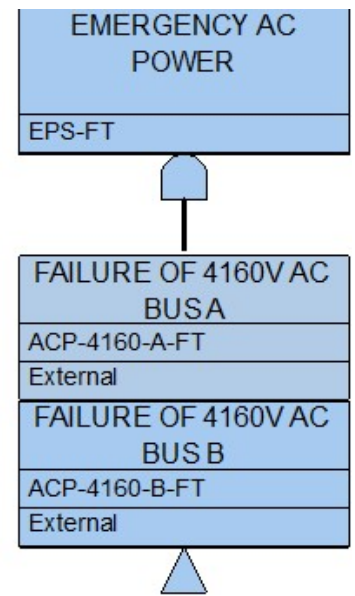

\section{EPS-RECP-FT}

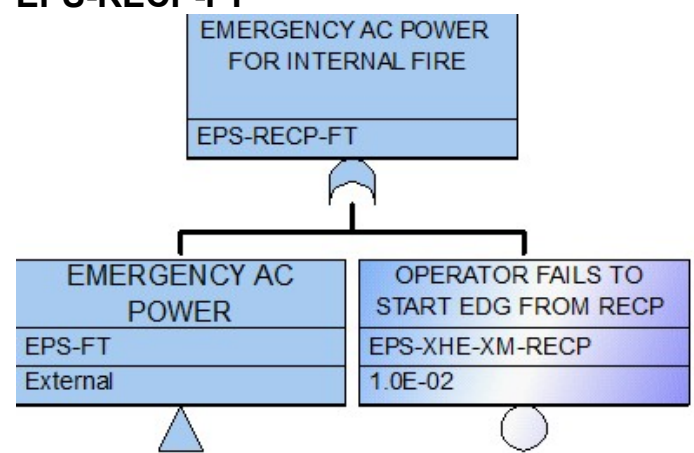

\section{EVAC}

\begin{tabular}{l} 
MCR ISNOT \\
EVACUATED \\
\hline EVAC
\end{tabular}

\section{F\&B-FT}

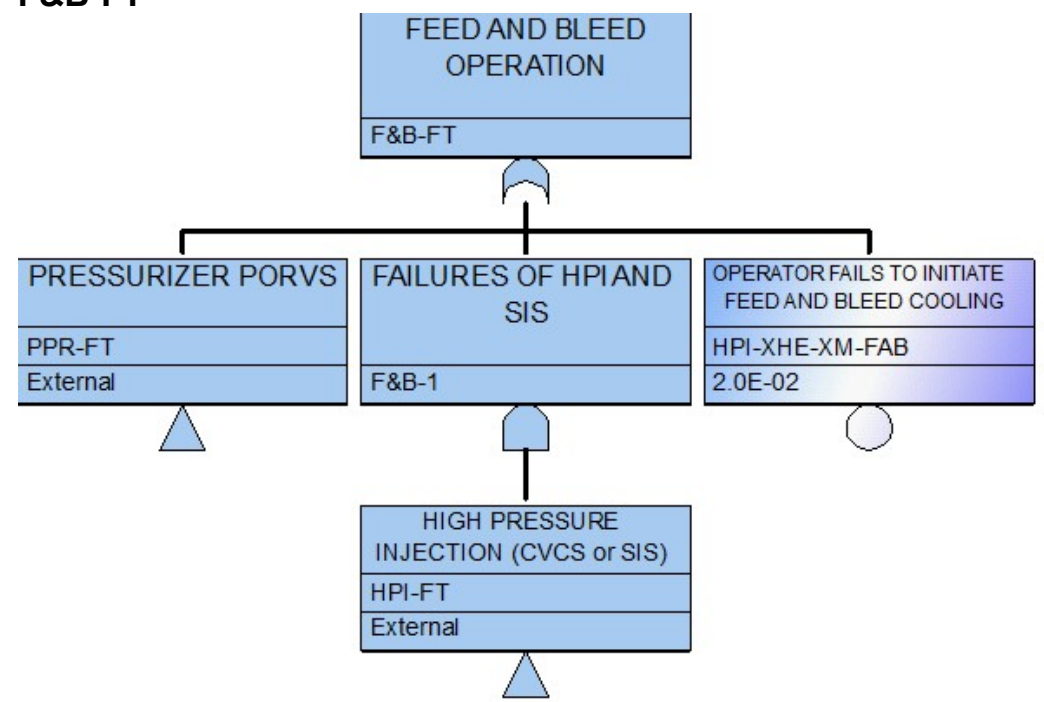

\section{F\&B-L-FT}


Generic Pressurized Water Reactor (PWR)

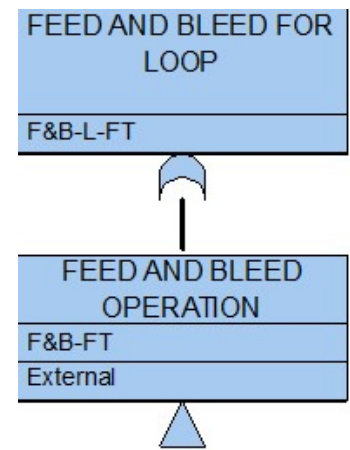

\section{FLEX-480}

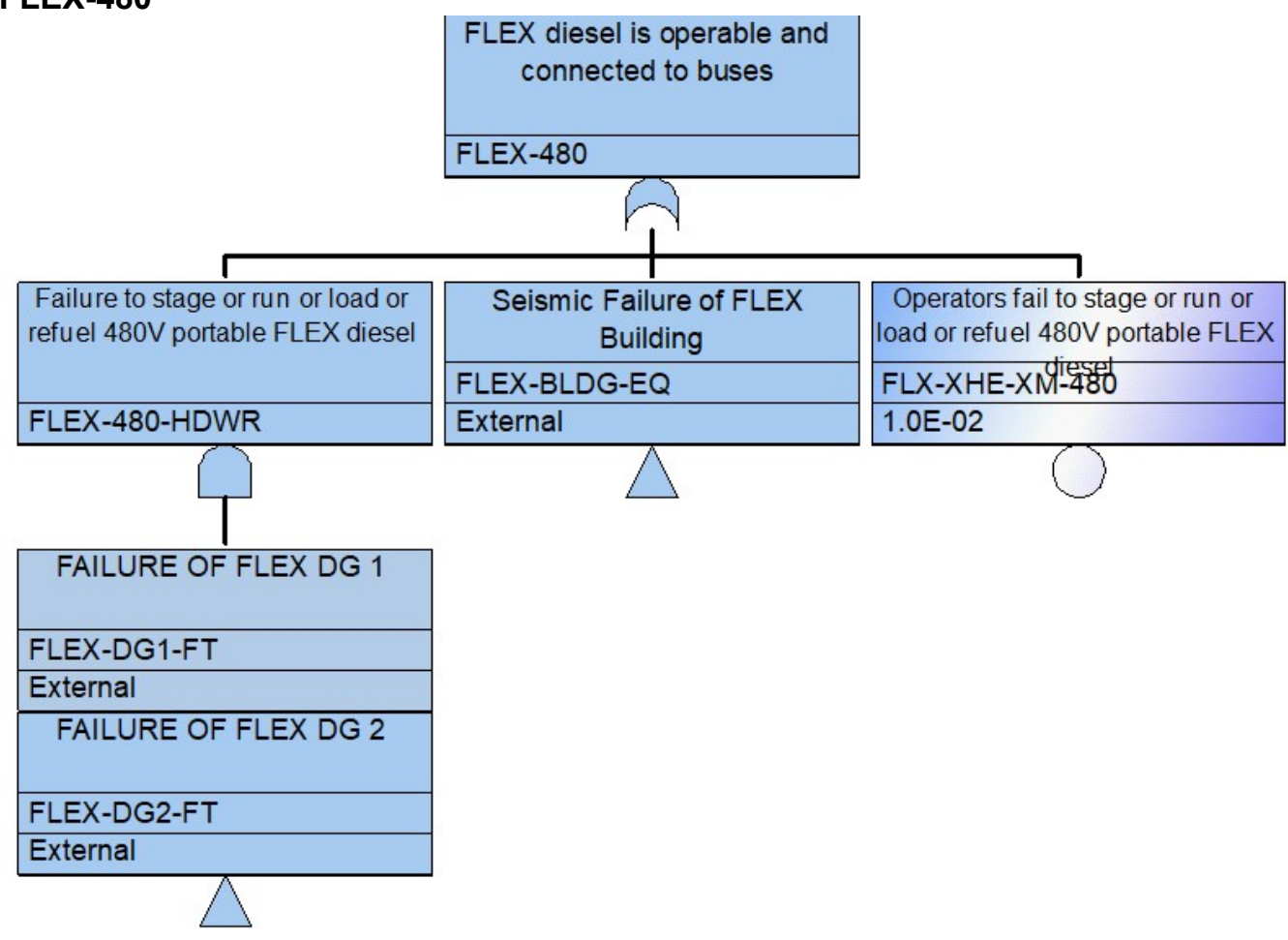

\section{FLEX-MUP}




\section{Generic Pressurized Water Reactor (PWR)}

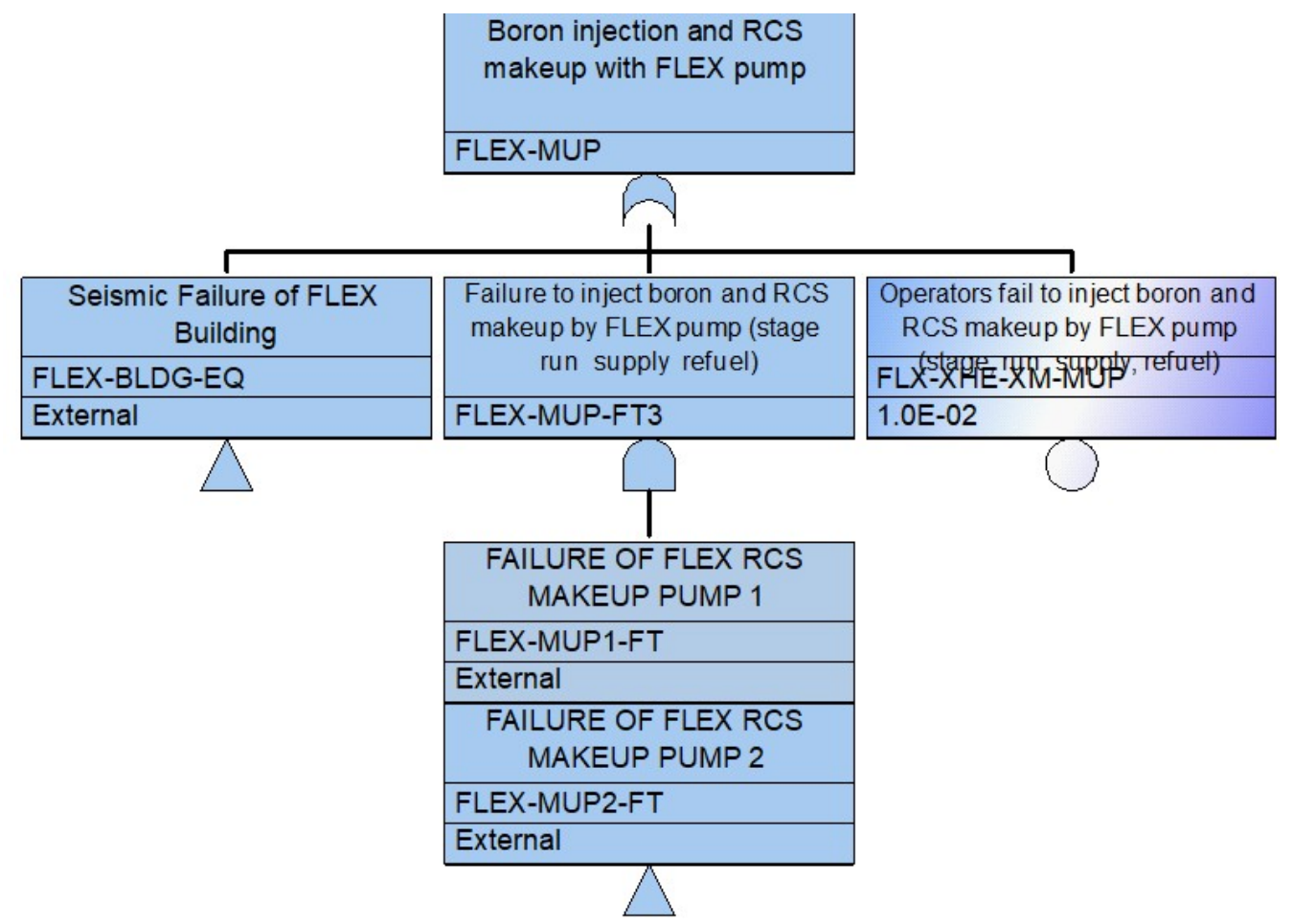

\section{FLEX-SGP}

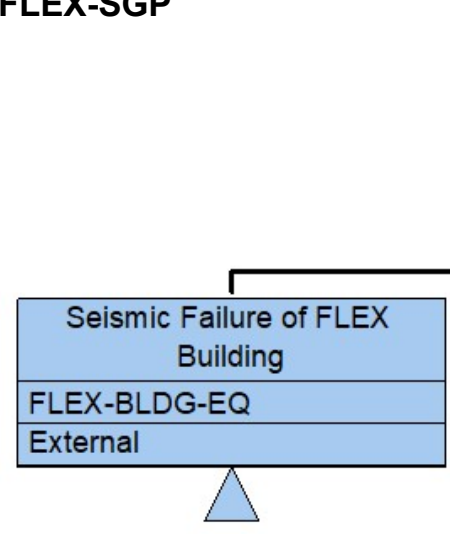

\section{FLI-TERM}

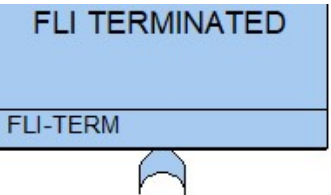

\section{FRI-SPUR-PORV}

FLEX SG pump is operable

FLEX-SGP

G)

\begin{tabular}{|l|}
\hline $\begin{array}{c}\text { Failure to stage or run or supply or } \\
\text { refill FLEX SG pump }\end{array}$ \\
\hline FLEX-SGP-HDWR \\
\hline $\begin{array}{c}\text { FAILURE OF FLEX SG } \\
\text { PUMP } 1\end{array}$ \\
\hline FLEX-SGP1-FT \\
\hline External \\
\hline $\begin{array}{c}\text { FAILURE OF FLEX SG } \\
\text { PUMP } 2\end{array}$ \\
\hline FLEX-SGP2-FT \\
\hline External \\
\hline
\end{tabular}

Failure of alternate water supply to FLEX-SG pump FLX-SGP-REFILL False

Operators fail to stage or run or supply or refill FLEX SG pump

FLX-XHE-XM-SGP

1.0E-02 
Generic Pressurized Water Reactor (PWR)

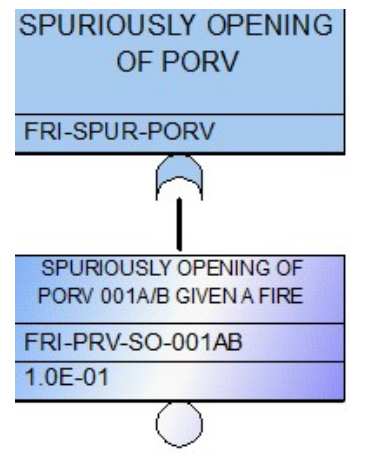

\section{HCN-WARN}

PLANT SHUTDOWN DUE TO HURRICANE WARNING

HCN-WARN

A

\section{HPI-FT}

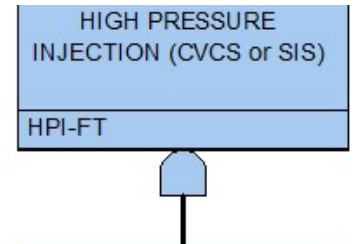

\begin{tabular}{|l|}
\hline \multicolumn{1}{|c|}{$\begin{array}{c}\text { CVC INJECTION } \\
\text { SEGMENT }\end{array}$} \\
\hline HPI-CVC-FT \\
\hline External \\
\hline $\begin{array}{c}\text { SAFETY INJECTION } \\
\text { SY STEM }\end{array}$ \\
\hline HPI-SIS-FT \\
\hline External \\
\hline
\end{tabular}

\section{HPI-HPR-L-FT}

HPI/HPR FUNCTIONS DURINGLOOP

HPI-HPR-L-FT

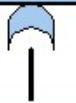

\begin{tabular}{|l|}
\hline $\begin{array}{c}\text { HIGH PRESSURE } \\
\text { INJECTION (CVCS or SIS) }\end{array}$ \\
\hline HPI-FT \\
\hline External \\
\hline HIGH PRESSURE \\
RECIRC \\
\hline HPR-FT \\
\hline External
\end{tabular}

HPI-L-FT 
Generic Pressurized Water Reactor (PWR)

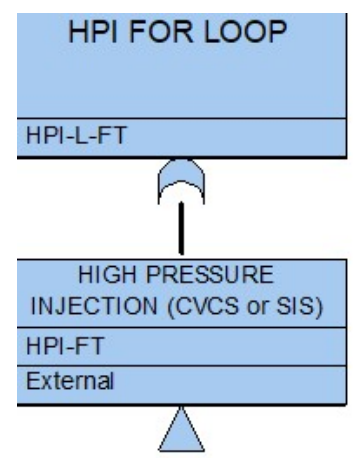

HPI-SIS-FT

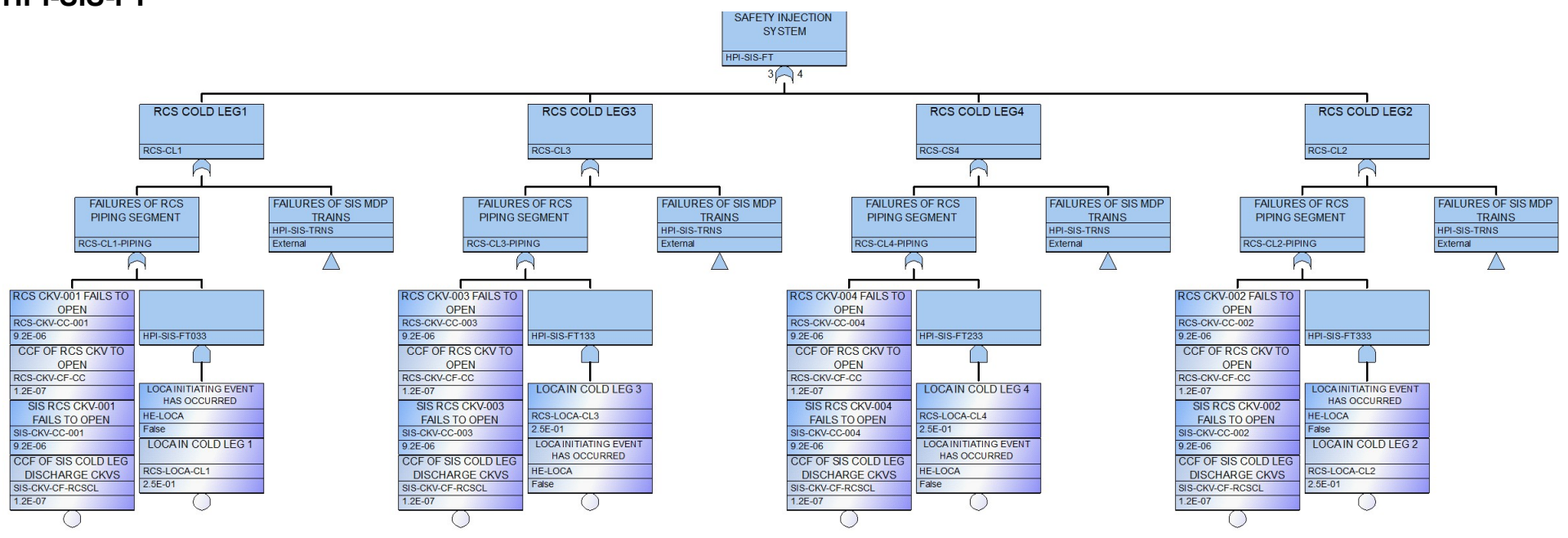

HPR-FT

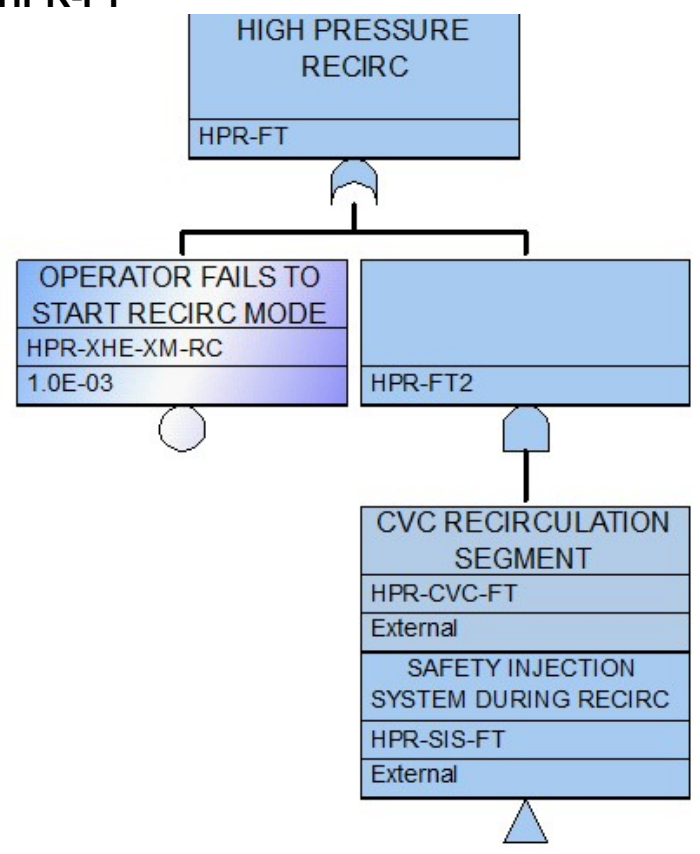

HPR-L-FT 


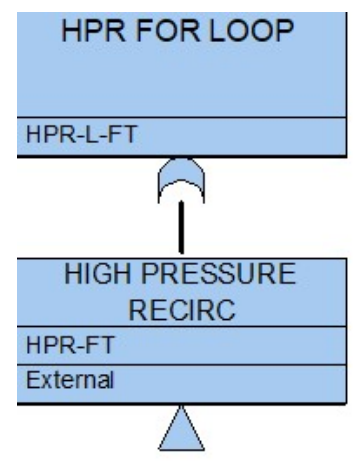

HPR-SIS-FT

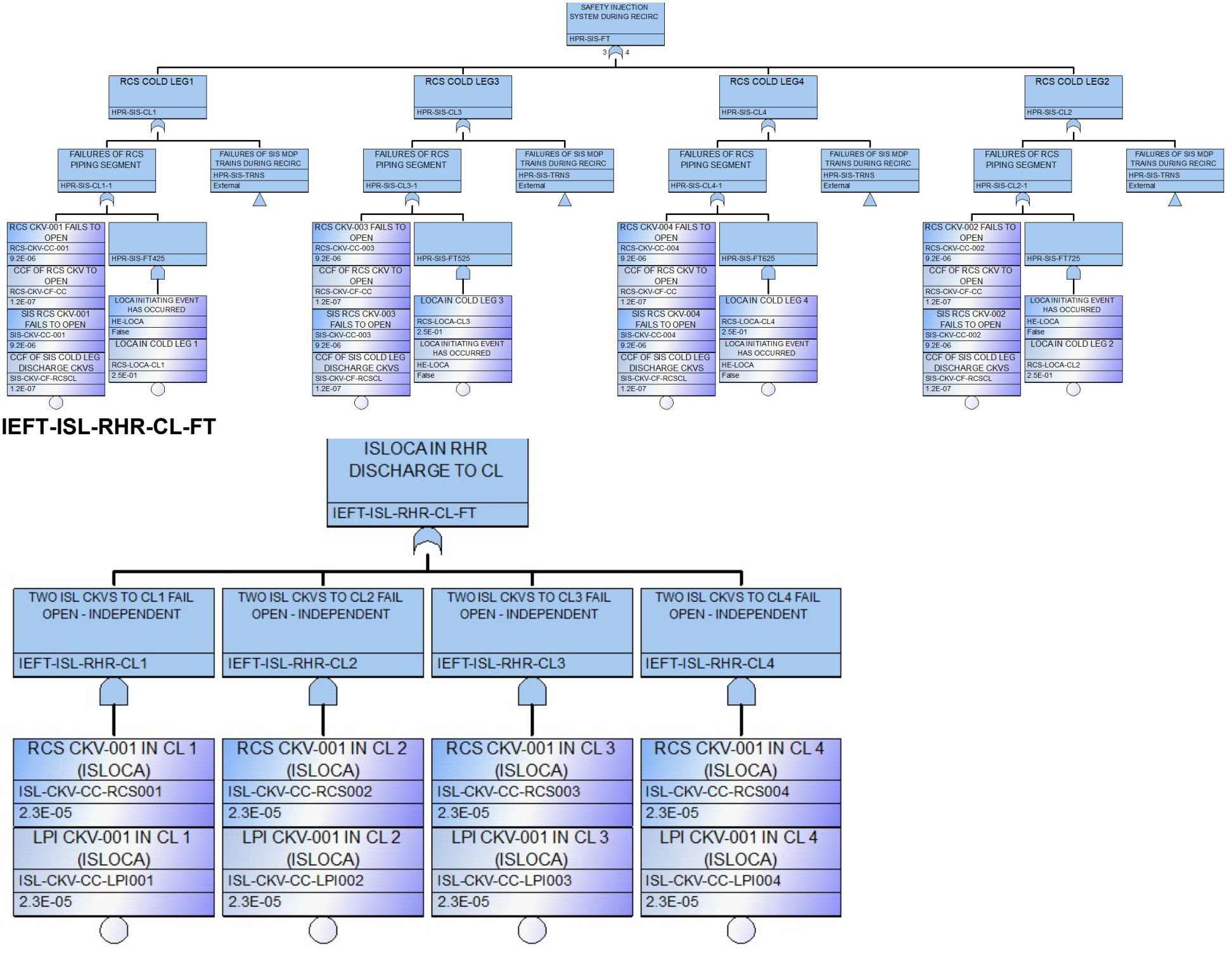

IEFT-ISL-RHR-HLS-FT 


\section{Generic Pressurized Water Reactor (PWR)}

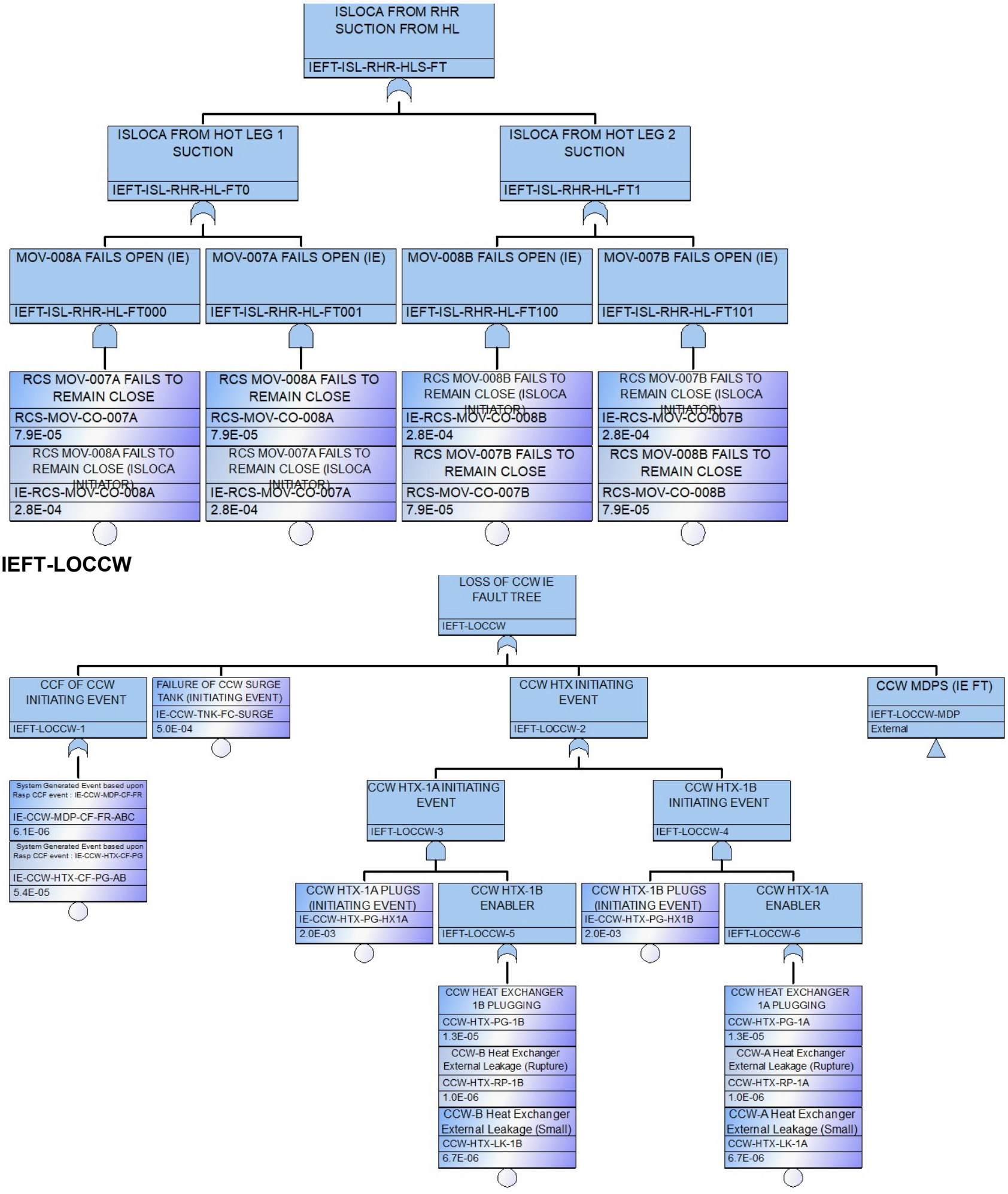

\section{ISL-DIAG-FT}




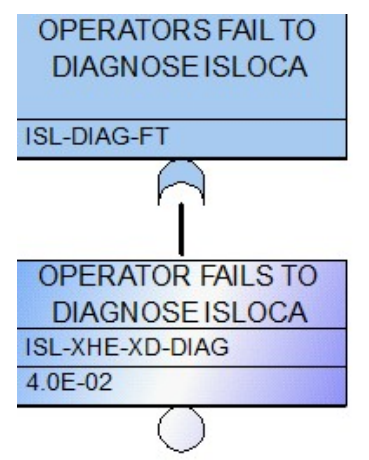

ISL-RHR-CLI-REC-FT

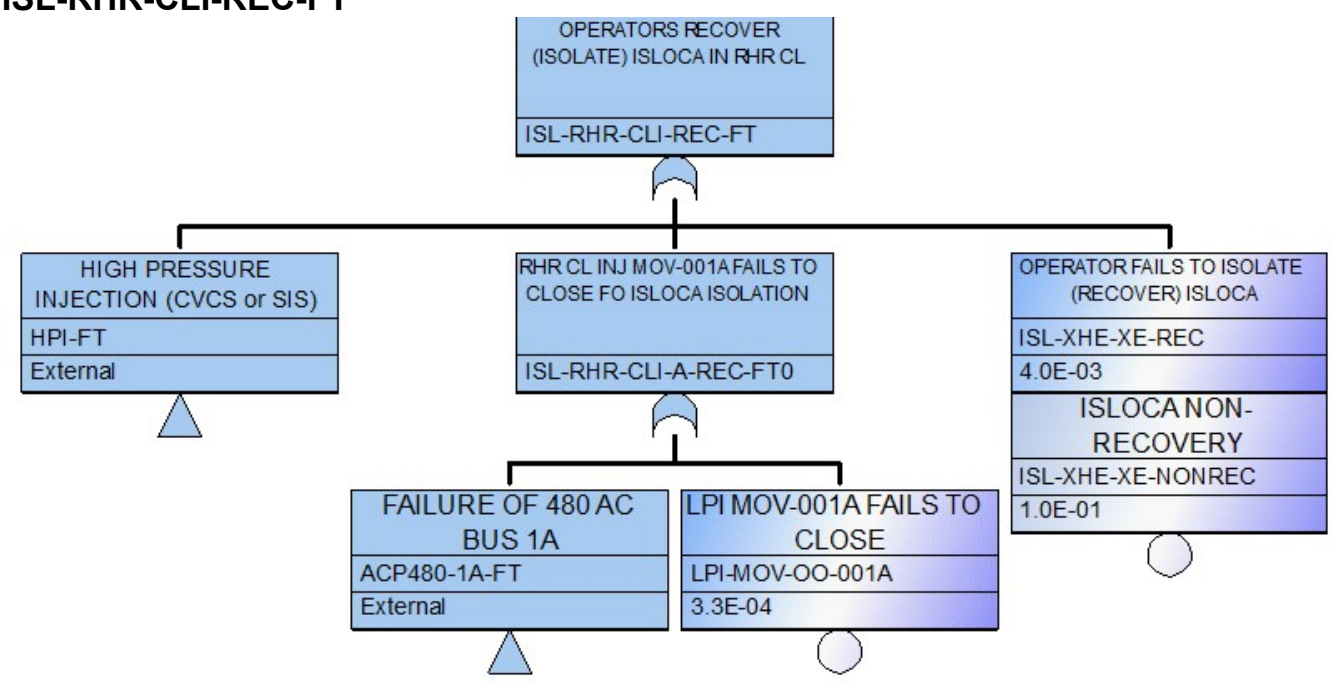

ISL-RHR-HL-REC-FT

\begin{tabular}{|c|}
\hline $\begin{array}{c}\text { OPERATORS RECOVER } \\
\text { (ISLOCARHR HL) }\end{array}$ \\
\hline $\begin{array}{c}\text { OPERATORFAILS TO ISOLATE } \\
\text { (RECOVER) ISLOCA }\end{array}$ \\
\hline ISL-XHE-XE-RECRHR \\
\hline 1.0E-01 \\
\hline ISLOCANON-REC-FT \\
RECOVERY \\
\hline ISL-XHE-XE-NRECRHR \\
\hline 1.0E-01 \\
\hline
\end{tabular}

ISL-RPT-LPI-FT
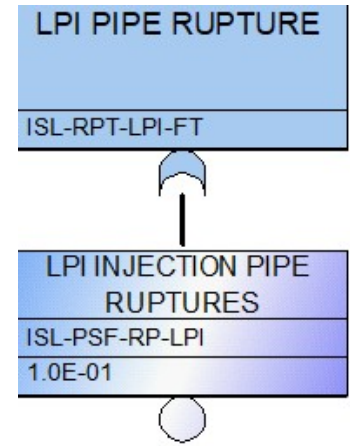

ISL-RPT-RHR-FT 
Generic Pressurized Water Reactor (PWR)

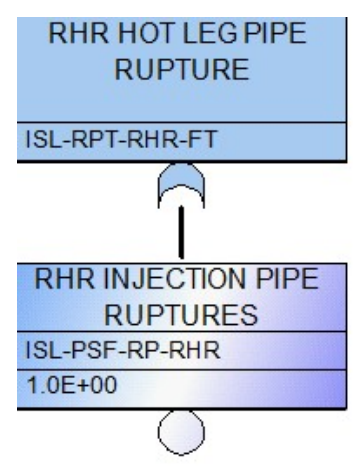

\section{LLOCA-EQ1-FT}

LLOCADUE TO

SEISMIC EVENT - BIN 1

LLOCA-EQ1-FT

(1)

LARGE LOCAOCCURS

SEISMIC BIN 1

RCS-SYS-EQ1-LLOCA

$1.5 \mathrm{E}-10$

LARGE LOCAOCCURS -SEISMIC BIN 1

RCS-RCP-EQ1-LLOCA

$1.0 \mathrm{E}-06$

LLOCA-EQ2-FT

LLOCADUETO

SEISMIC EVENT - BIN 2

LLOCA-EQ2-FT

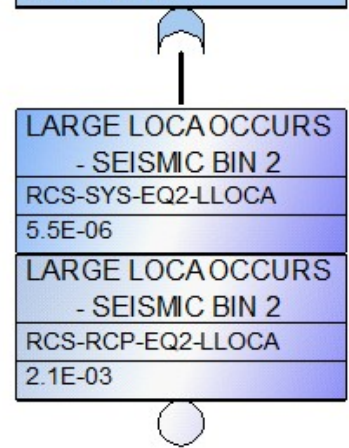

LLOCA-EQ3-FT

\begin{tabular}{|c|} 
LLOCA DUE TO \\
SEISMIC EVENT - BIN 3 \\
\hline LLOCA-EQ3-FT \\
\hline
\end{tabular}

\begin{tabular}{|l|}
\hline $\begin{array}{c}\text { LARGE LOCAOCCURS } \\
\text {-SEISMIC BIN } 3\end{array}$ \\
\hline RCS-SYS-EQ3-LLOCA \\
\hline 4.5E-04 \\
\hline $\begin{array}{c}\text { LARGE LOCAOCCURS } \\
\text { - SEISMIC BIN } 3\end{array}$ \\
\hline RCS-RCP-EQ3-LLOCA \\
\hline $3.8 E-02$ \\
\hline
\end{tabular} 


\section{LLOCA-EQ4-FT \\ LLOCADUE TO \\ SEISMIC EVENT - BIN 4 \\ LLOCA-EQ4-FT \\ LARGE LOCAOCCURS \\ SEISMIC BIN 4 \\ RCS-SYS-EQ4-LLOCA \\ 6.2E-03 \\ LARGE LOCAOCCURS - SEISMIC BIN 4 \\ RCS-RCP-EQ4-LLOCA \\ 1.7E-01}

\section{LLOCA-EQ5-FT}

\begin{tabular}{|c|} 
LLOCA DUE TO \\
SEISMIC EVENT - BIN 5 \\
\hline LLOCA-EQ5-FT \\
\hline
\end{tabular}

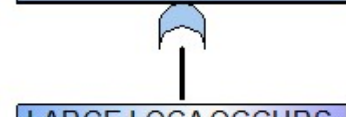

\begin{tabular}{|l|}
\hline $\begin{array}{c}\text { LARGE LOCAOCCURS } \\
\text { - SEISMIC BIN } 5\end{array}$ \\
\hline RCS-SYS-EQ5-LLOCA \\
\hline 6.2E-03 \\
\hline $\begin{array}{c}\text { LARGE LOCAOCCURS } \\
\text { - SEISMIC BIN } 5\end{array}$ \\
\hline RCS-RCP-EQ5-LLOCA \\
\hline $1.7 \mathrm{E}-01$ \\
\hline
\end{tabular}

\section{LLOCA-EQ6-FT}

LLOCADUETO

SEISMIC EVENT - BIN 6

LLOCA-EQ6-FT

(1)

LARGE LOCAOCCURS - SEISMIC BIN 6

RCS-SYS-EQ6-LLOCA

4.6E-02

LARGE LOCAOCCURS - SEISMIC BIN 6

RCS-RCP-EQ6-LLOCA

4.4E-01

LOOP-EQ1-FT 
Generic Pressurized Water Reactor (PWR)

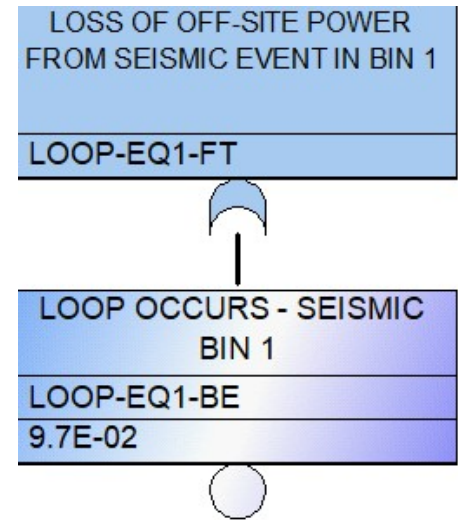

\section{LOOP-EQ2-FT}

LOSS OF OFFSITE POWER

FROM SEISMIC EVENT IN BIN 2
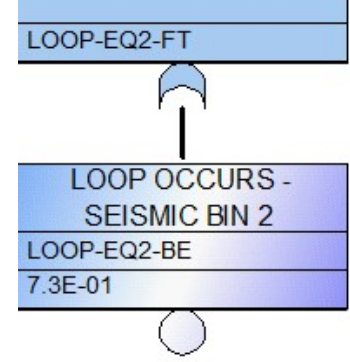

\section{LOOP-EQ3-FT}

LOSS OF OFF-SITE POWER

FROM SEISMIC EVENT IN BIN 3

LOOP-EQ3-FT

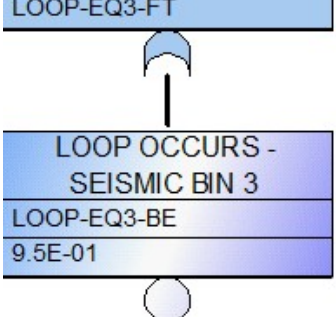

LOOP-EQ4-FT

LOSS OF OFF-SITE POWER

FROM SEISMIC EVENT IN BIN 4

LOOP-EQ4-FT

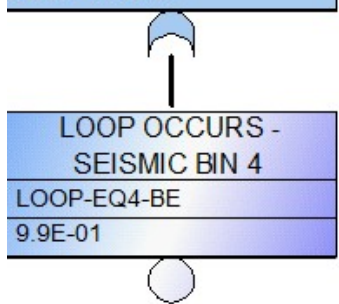

LOOP-EQ5-FT 
Generic Pressurized Water Reactor (PWR)

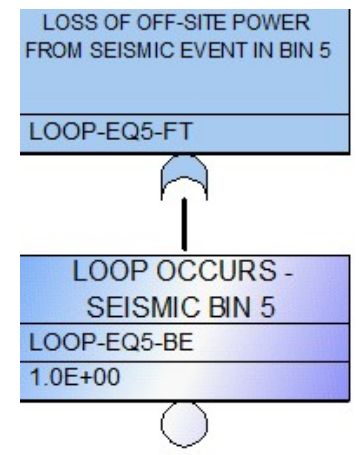

\section{LOOP-EQ6-FT}

LOSS OF OFF-SITE POWER

FROM SEISMIC EVENT IN BIN 6

LOOP-EQ6-FT

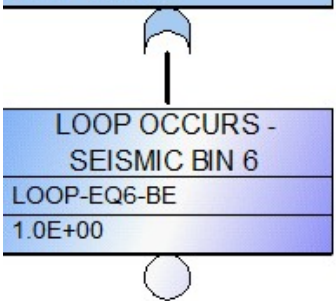

\section{LOOP-HCN1}

LOOP DUE TO

HURRICANE BIN 1

LOOP-HCN1

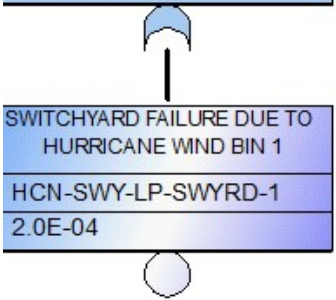

\section{LOOP-HCN2}

LOOP DUE TO

HURRICANE BIN 2

LOOP-HCN2

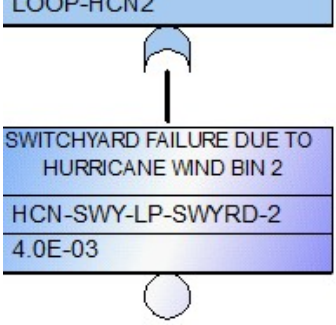

LOOP-HCN3 
Generic Pressurized Water Reactor (PWR)

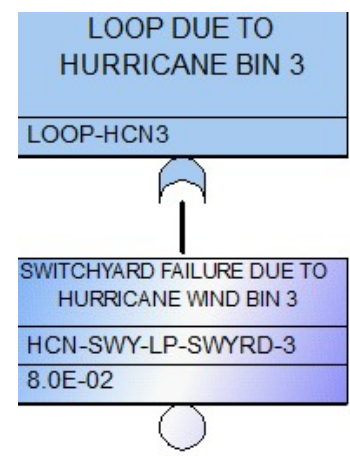

\section{LOOP-HCN4}

LOOP DUE TO

HURRICANE BIN 4

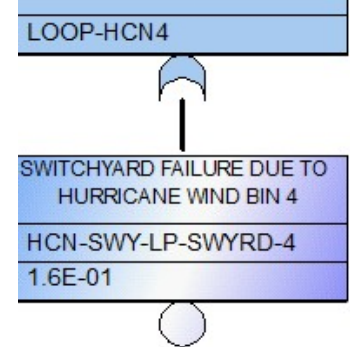

\section{LOOP-HWD}

LOOP DUE TO $136 \mathrm{MPH}$ HIGH WIND EVENT

LOOP-HWD

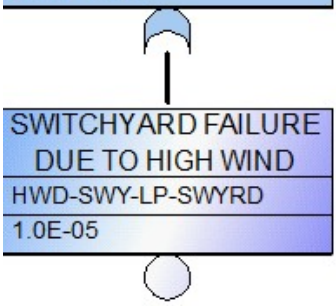

\section{LOOP-TOR1}

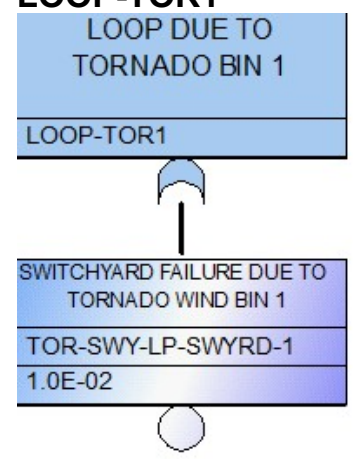

LOOP-TOR2 
Generic Pressurized Water Reactor (PWR)

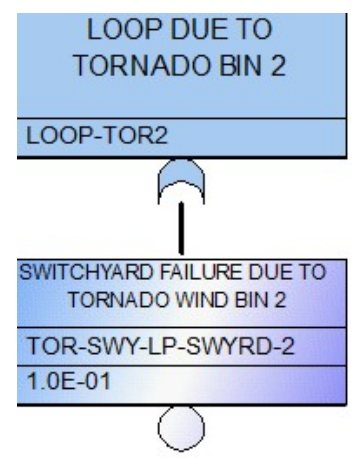

\section{LOOP-TOR3}

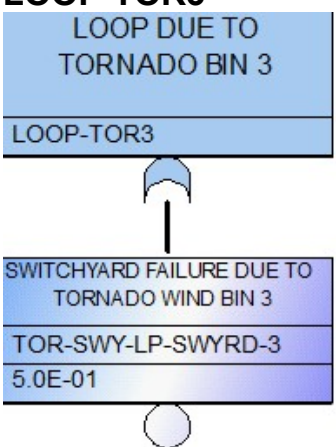

\section{LOSC-FT}

\begin{tabular}{|l|}
\hline \\
COOLING \\
\hline COSC-FT \\
\hline CCW-FT AAND B \\
\hline External \\
\hline $\begin{array}{l}\text { CHEMICALAND VOLUME } \\
\text { CONTROLSYSTEM }\end{array}$ \\
\hline CVC-FT \\
\hline External \\
\hline
\end{tabular}

\section{LOSC-L-FT}
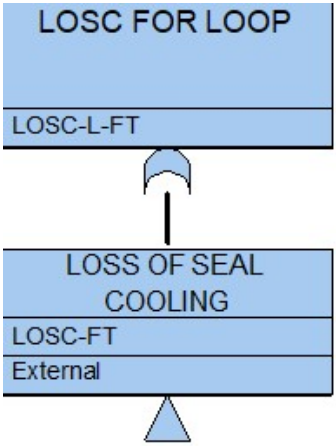

\section{LPI-FT}




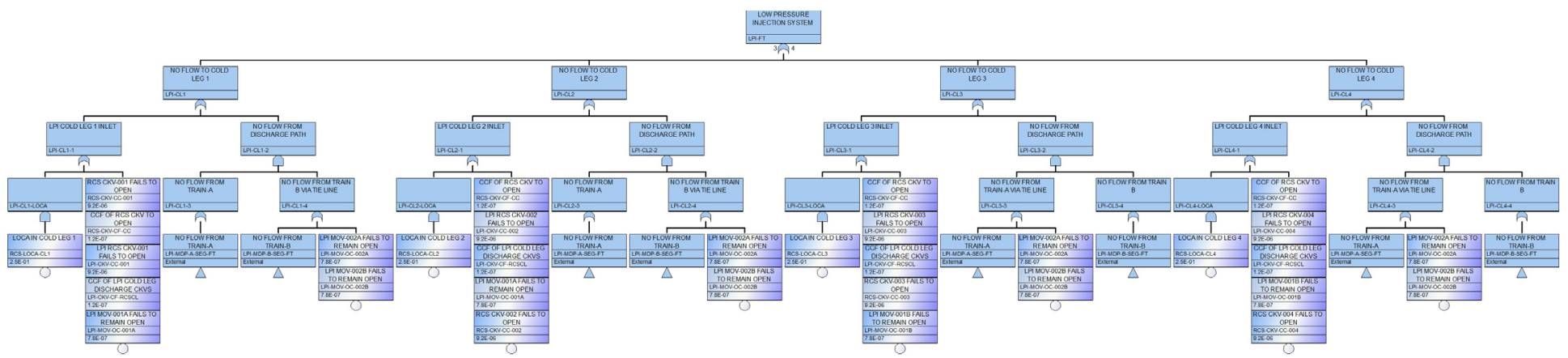

\section{LPR-FT}

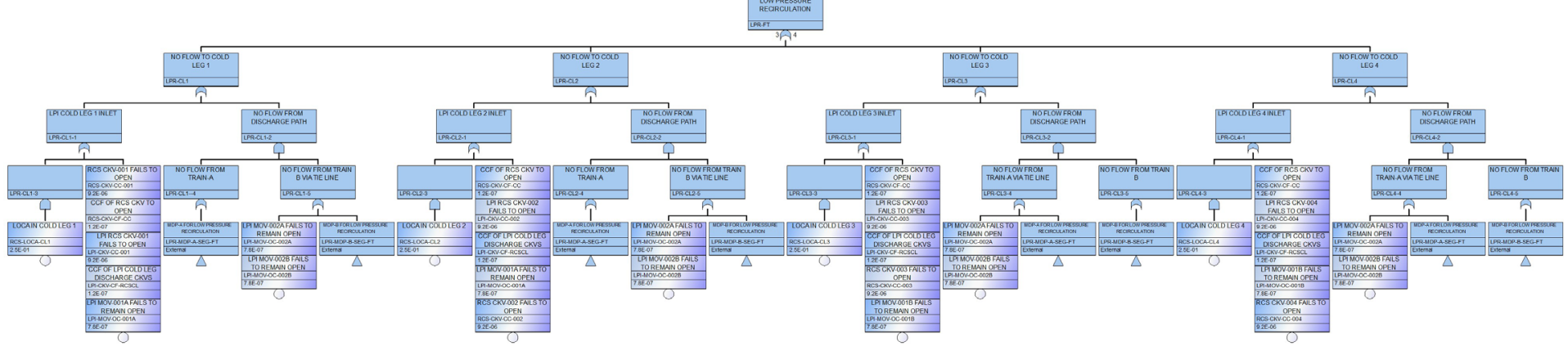

\section{MFW-FT}

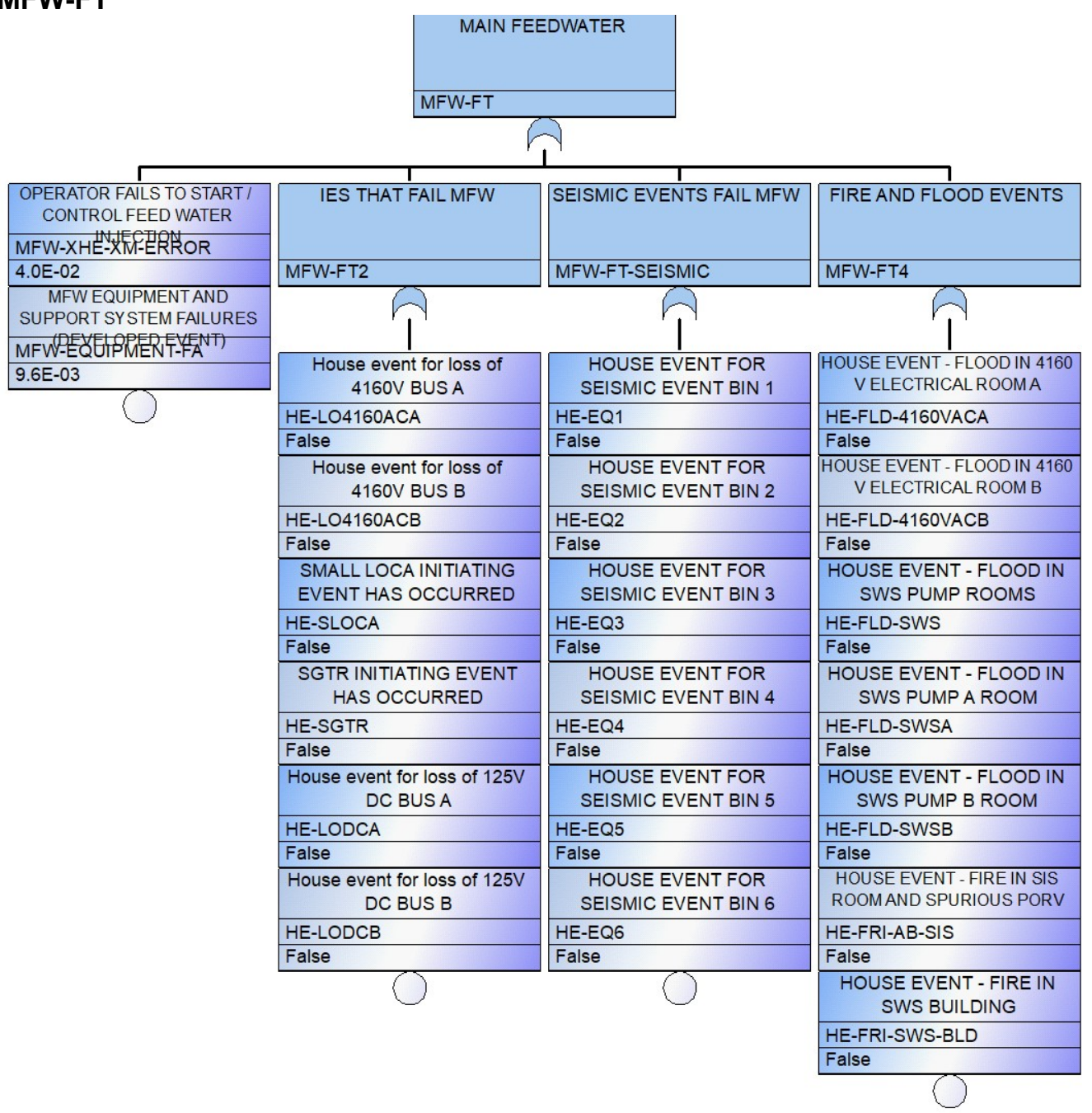

\section{MSI-FT}




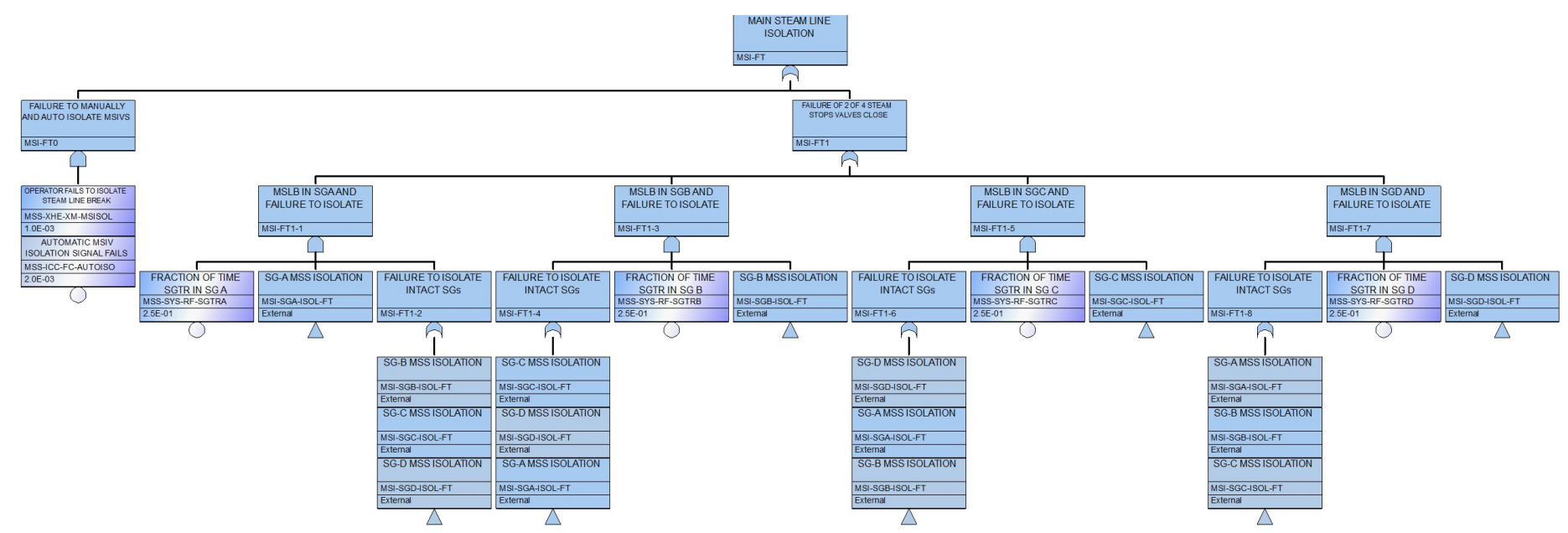

\section{MUT-EX}

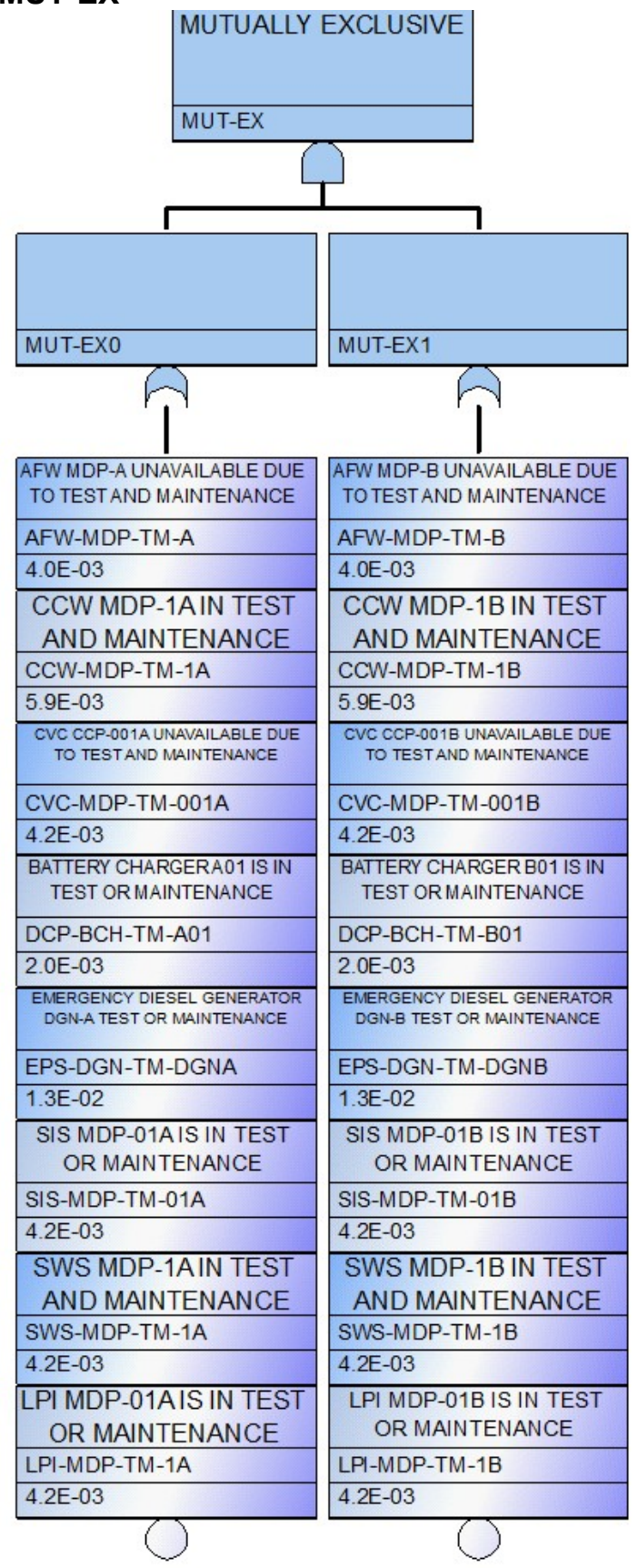


Generic Pressurized Water Reactor (PWR)

\section{OPR-01H-FT}

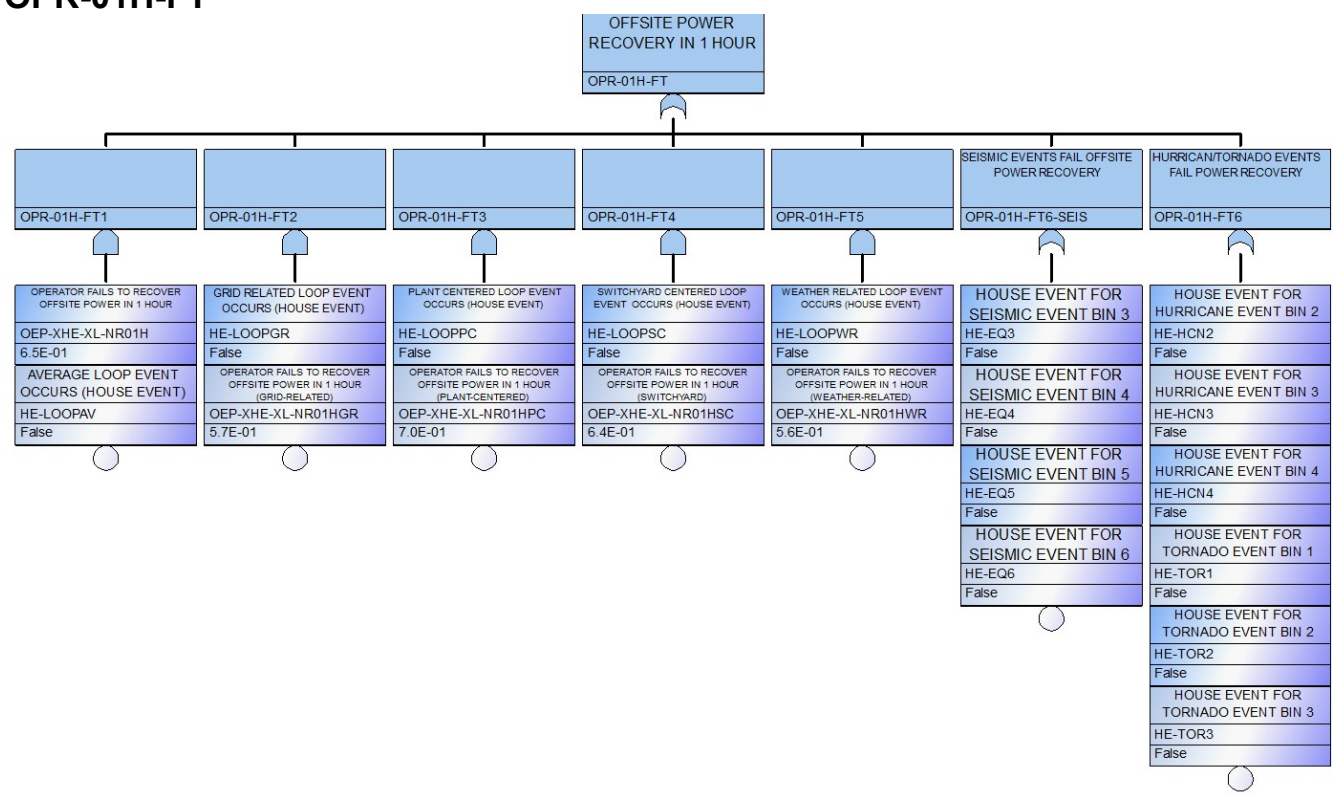

OPR-02H-FT

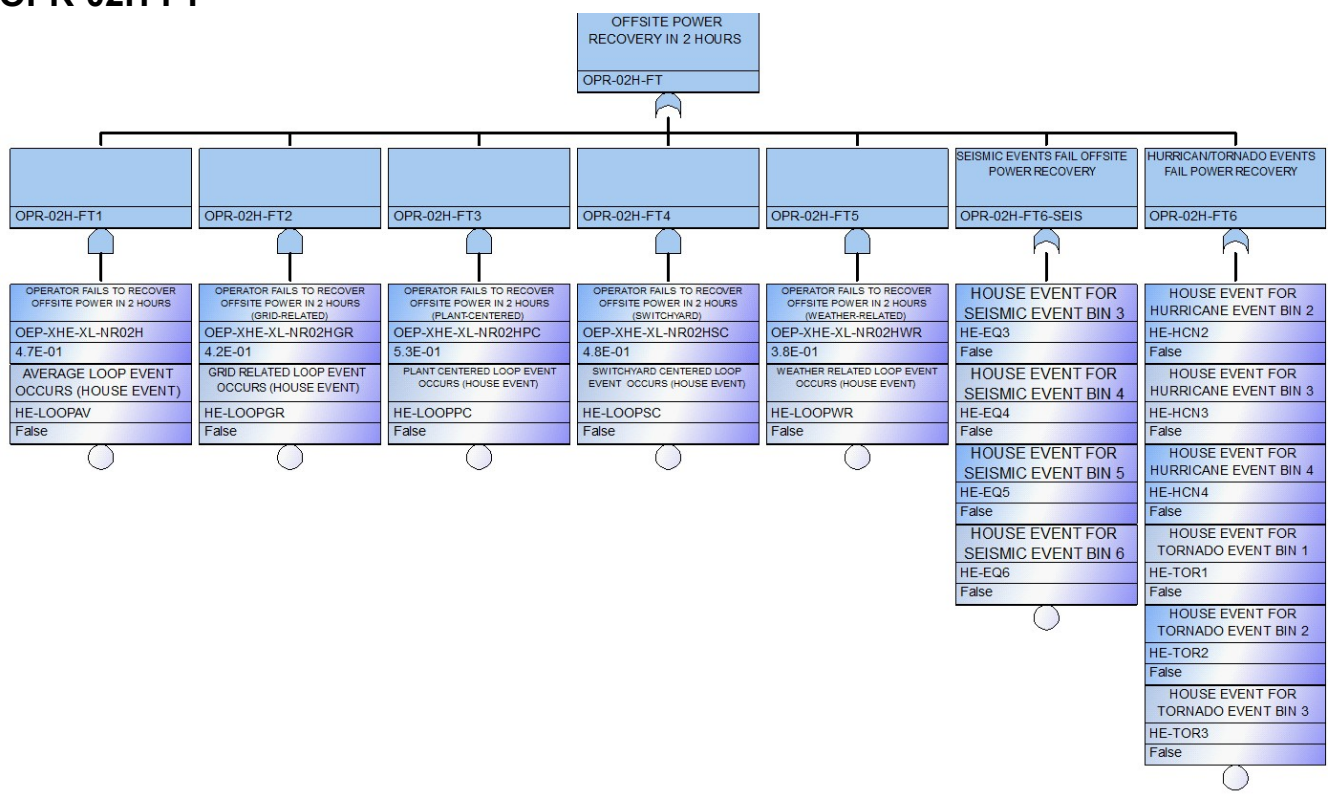

OPR-08H-FT

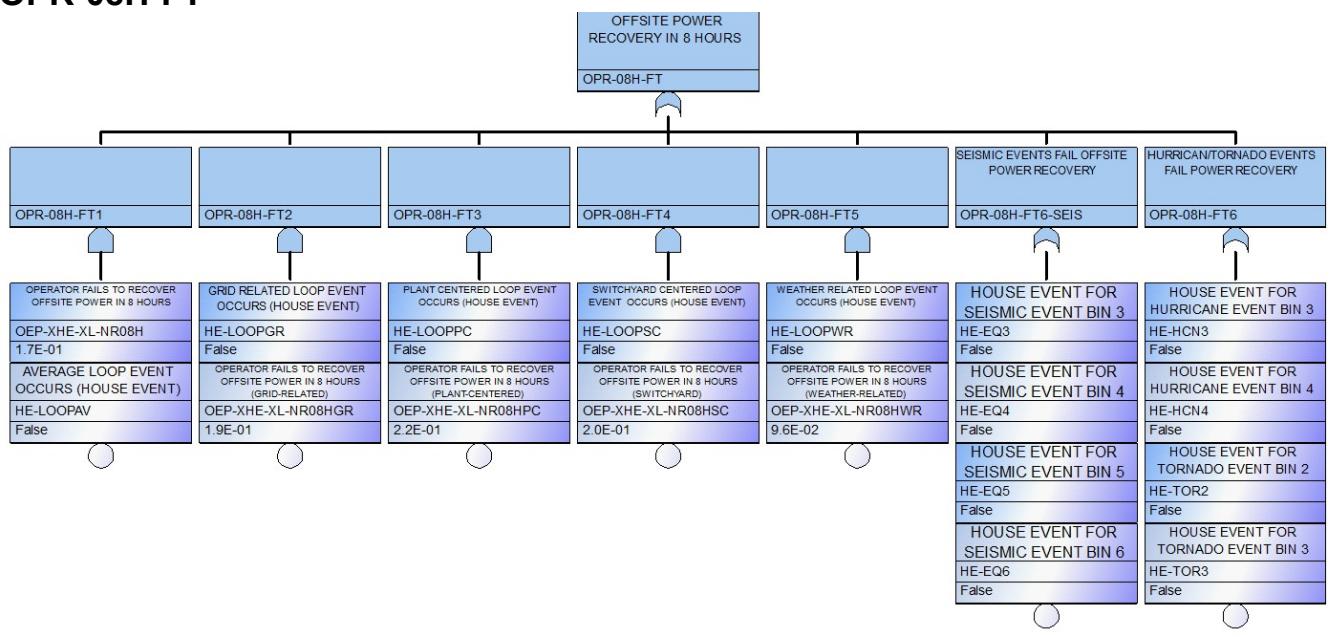




\section{Generic Pressurized Water Reactor (PWR)}

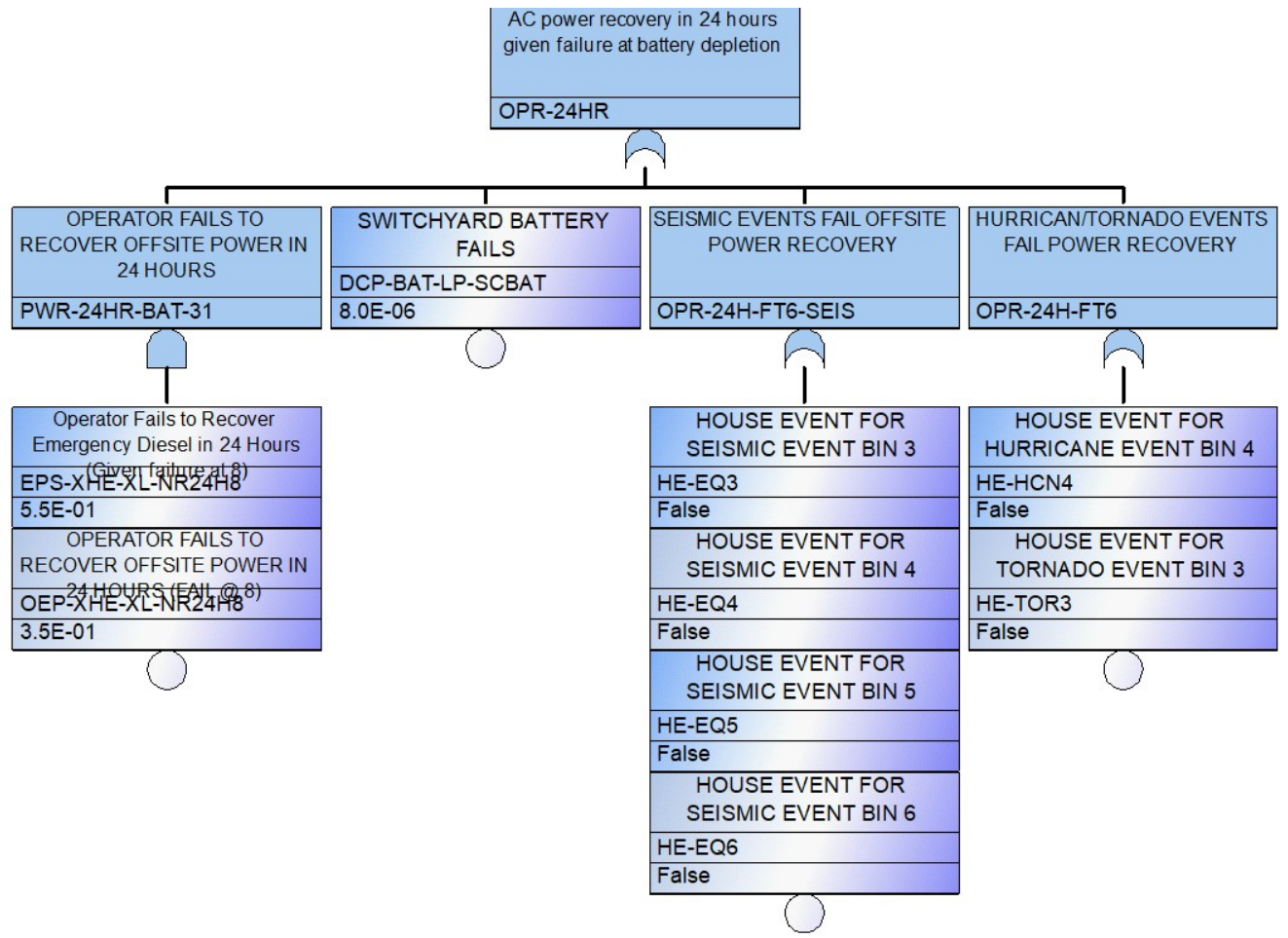

\section{OPR-72HR}

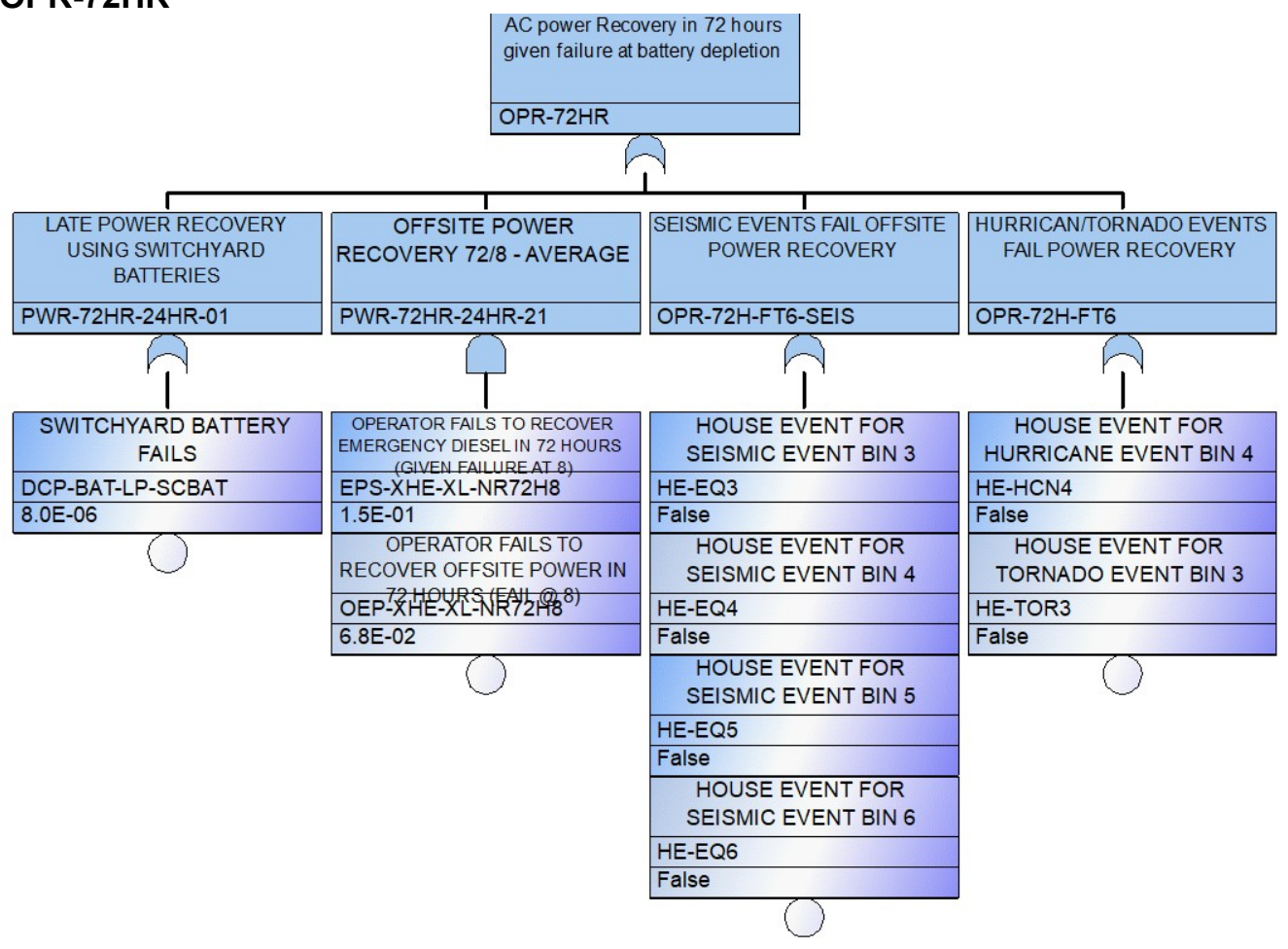

PORV-1-FT 
Generic Pressurized Water Reactor (PWR)

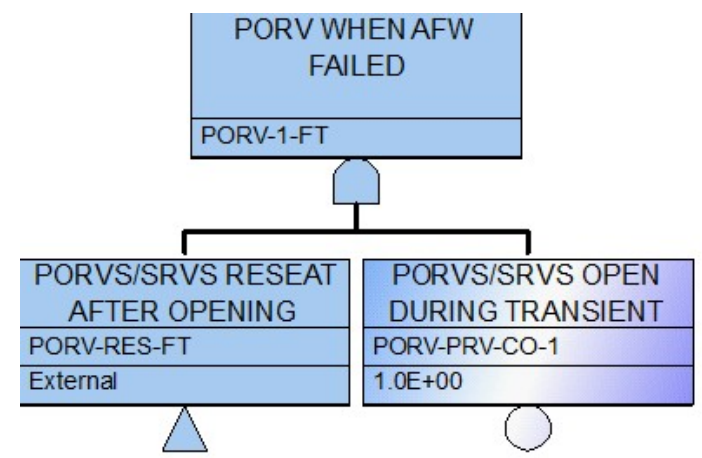

PORV-A-FT

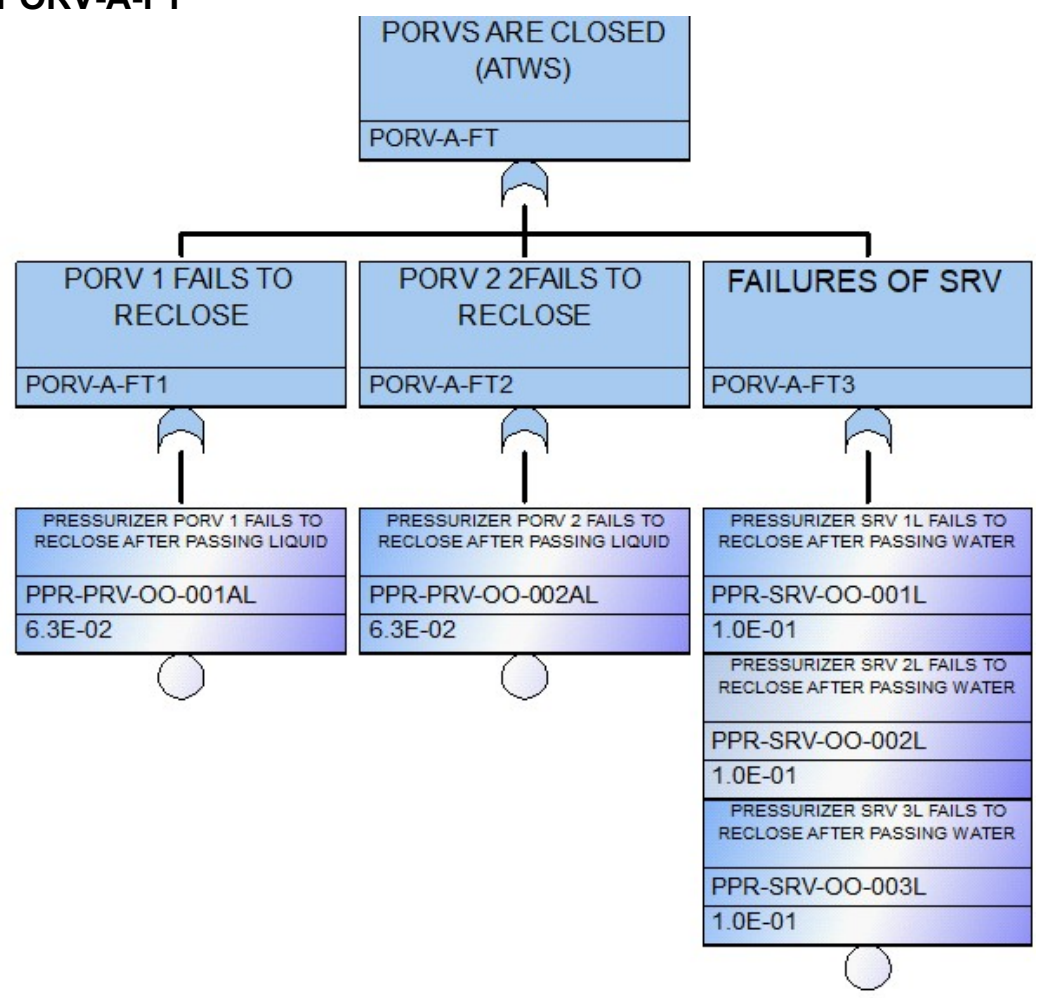

PORV-B-FT
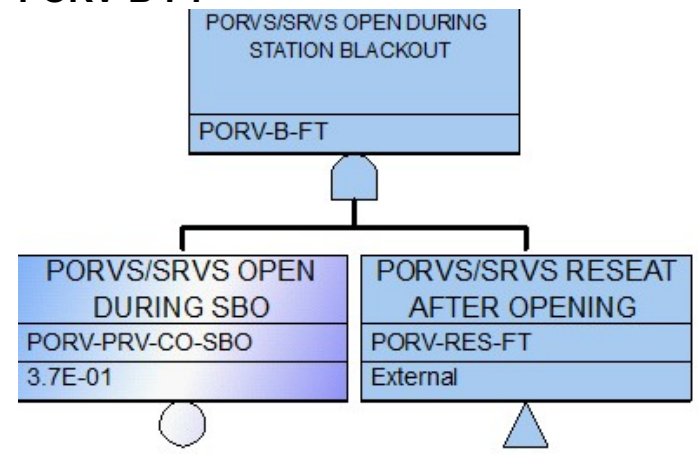

PORV-FT 
Generic Pressurized Water Reactor (PWR)

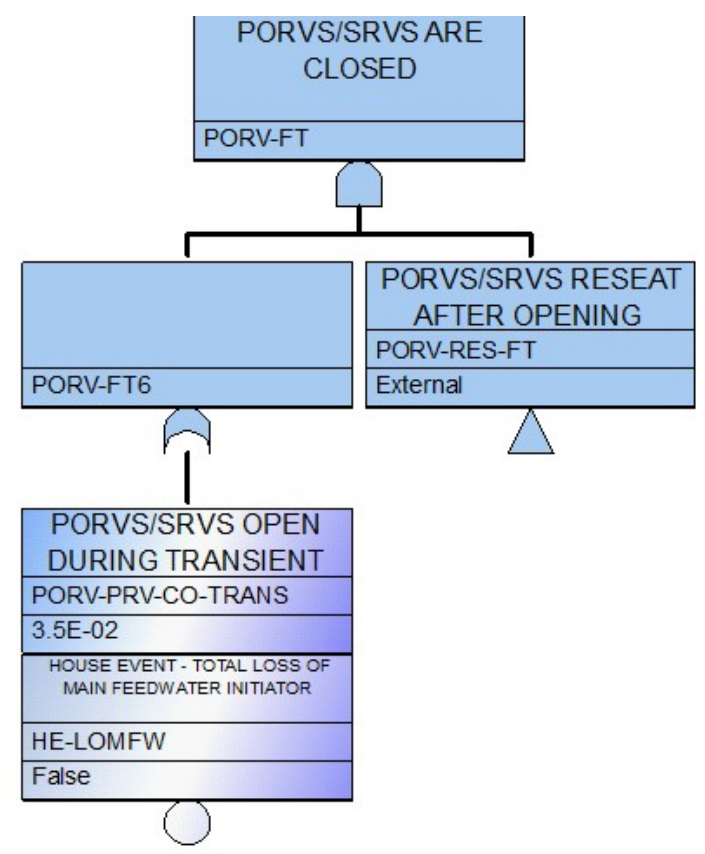

PORV-L-FT

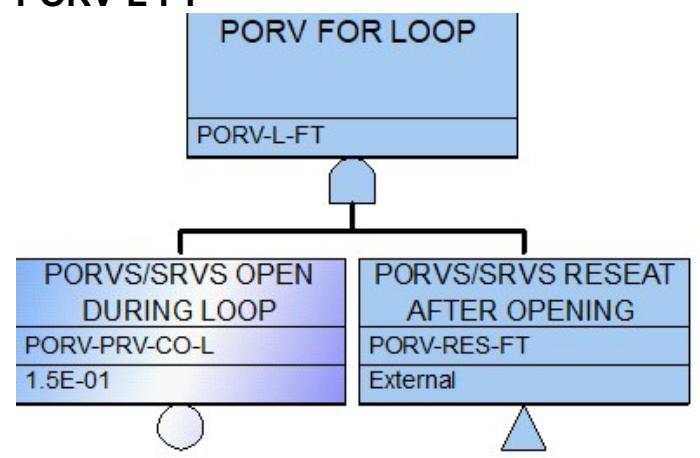

\section{PZR-FT}

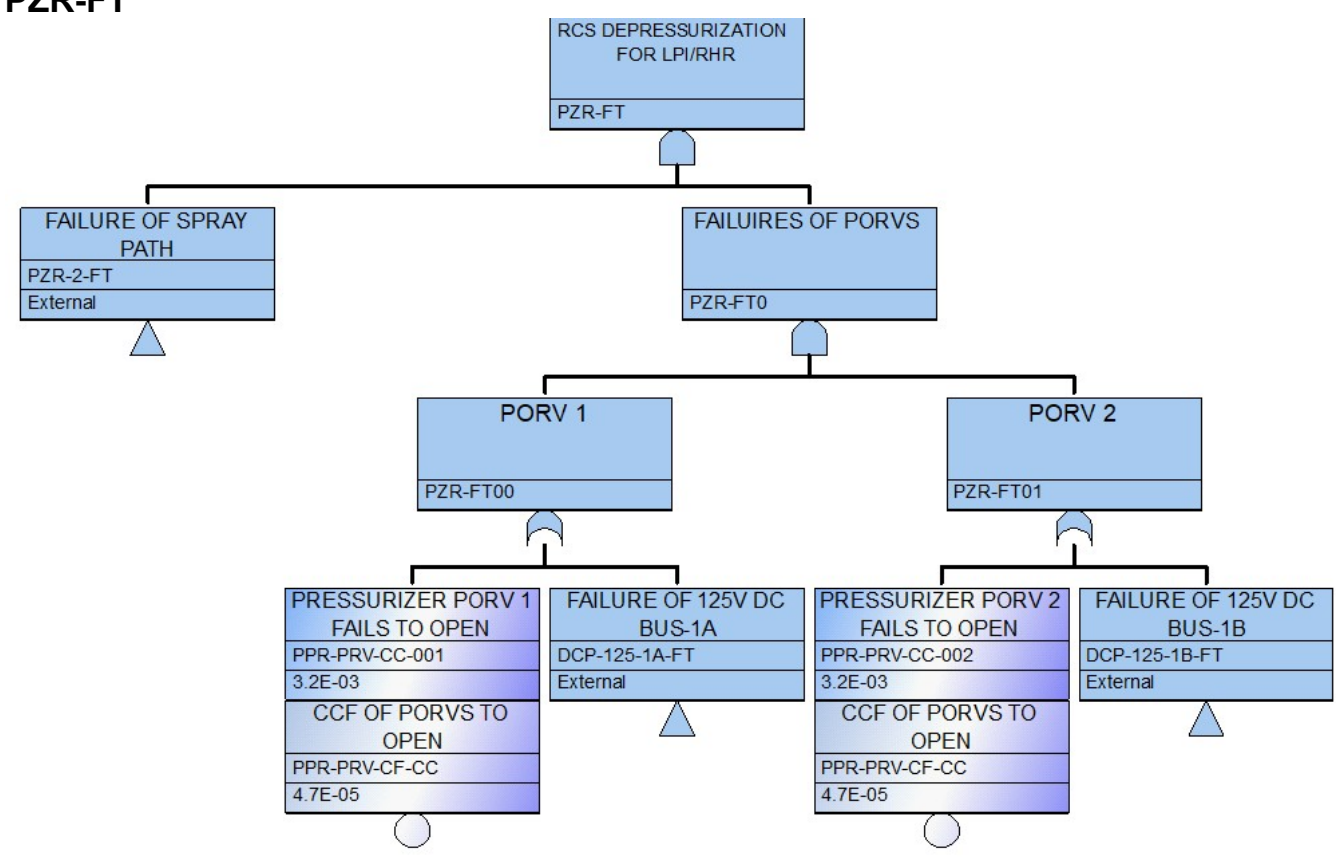

RCPSLOCA-FT 


\section{Generic Pressurized Water Reactor (PWR)}

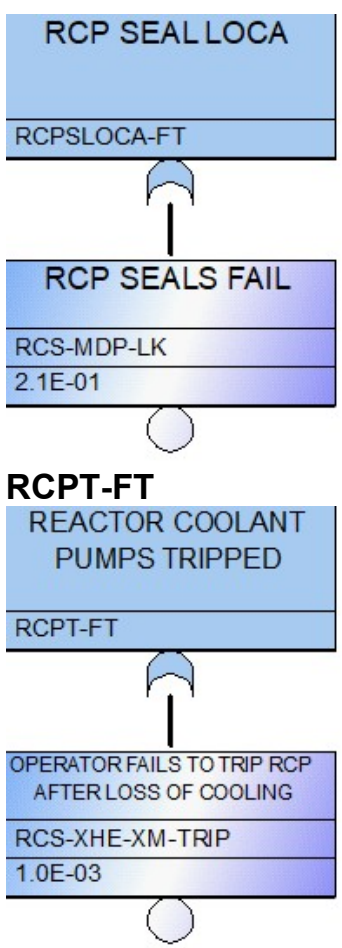

\section{RCSPRESS-FT}

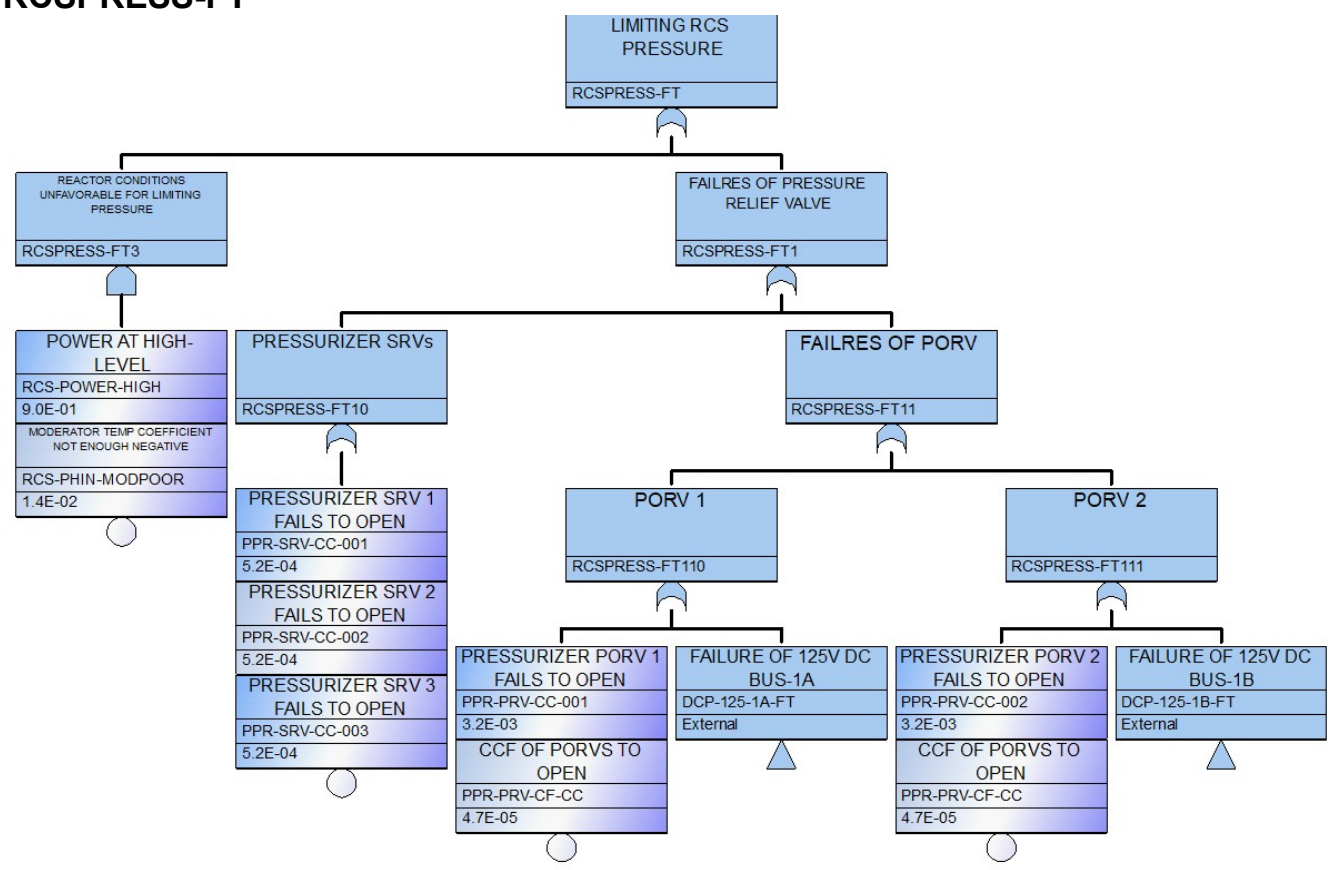

\section{REFILL1}

RWST REFILL

REFILL1

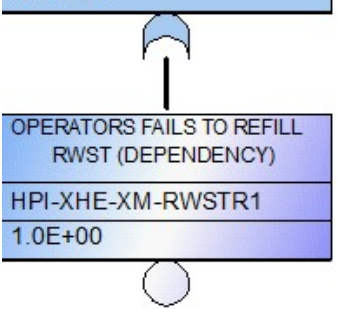

\section{REFILL-FT}

Model Version: 1.0 
Generic Pressurized Water Reactor (PWR)

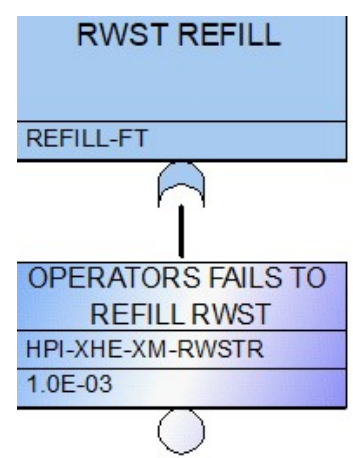

\section{RHR-FT}

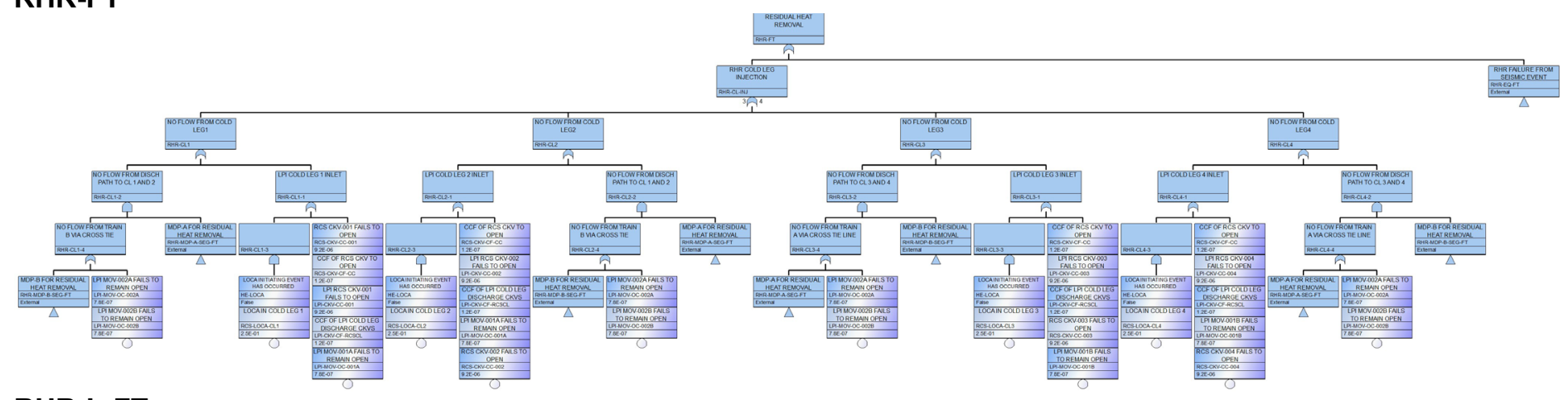

\section{RHR-L-FT}

RESIDUALHEAT

REMOVAL FOR LOOP

RHR-L-FT

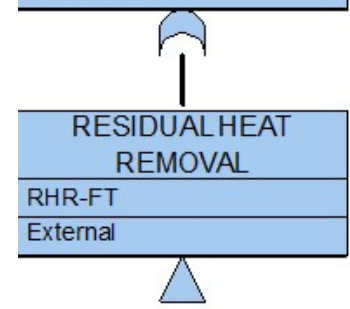

RPS-FT 


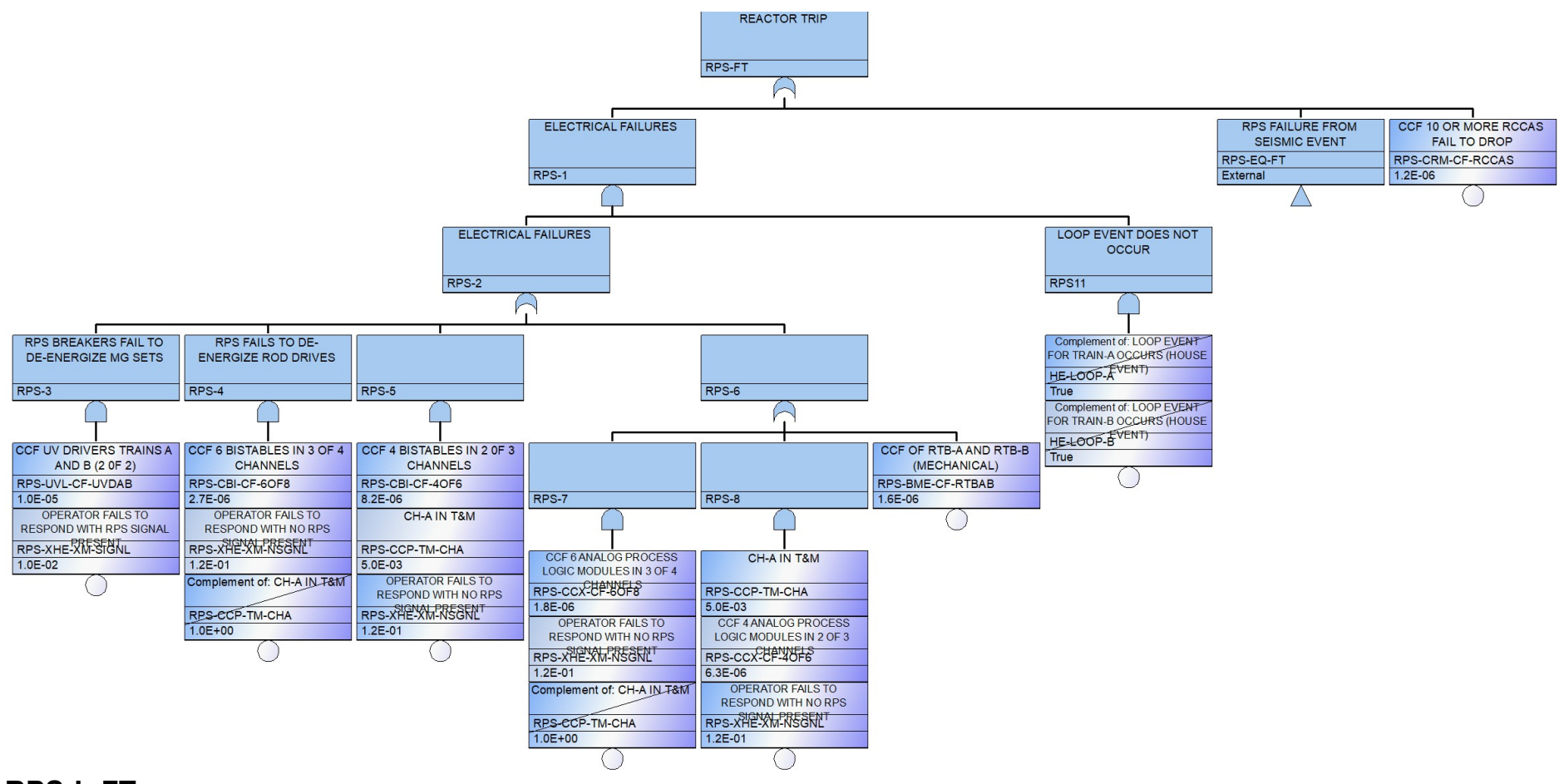

\section{RPS-L-FT}

\begin{tabular}{l}
$\begin{array}{c}\text { REACTOR TRIP FOR } \\
\text { IE-LOOP }\end{array}$ \\
\hline RPS-L-FT \\
\hline RPS-FT \\
\hline External
\end{tabular}

\section{RSD-B-FT}

\begin{tabular}{c} 
RAPID SECONDARY \\
DEPRESS \\
\hline RSD-B-FT \\
\hline
\end{tabular}

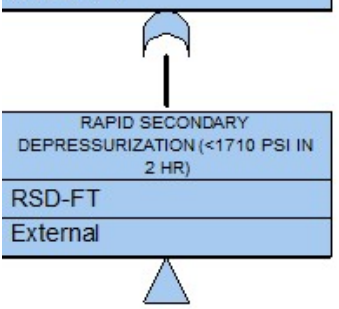

\section{RSD-FT}

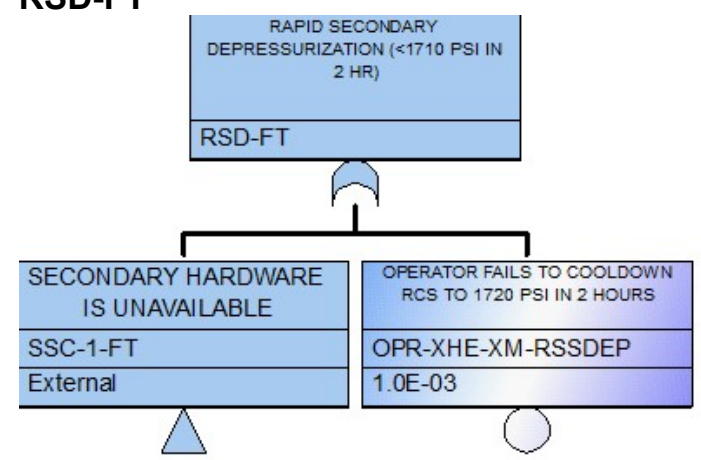


RWST-TNK-HWD

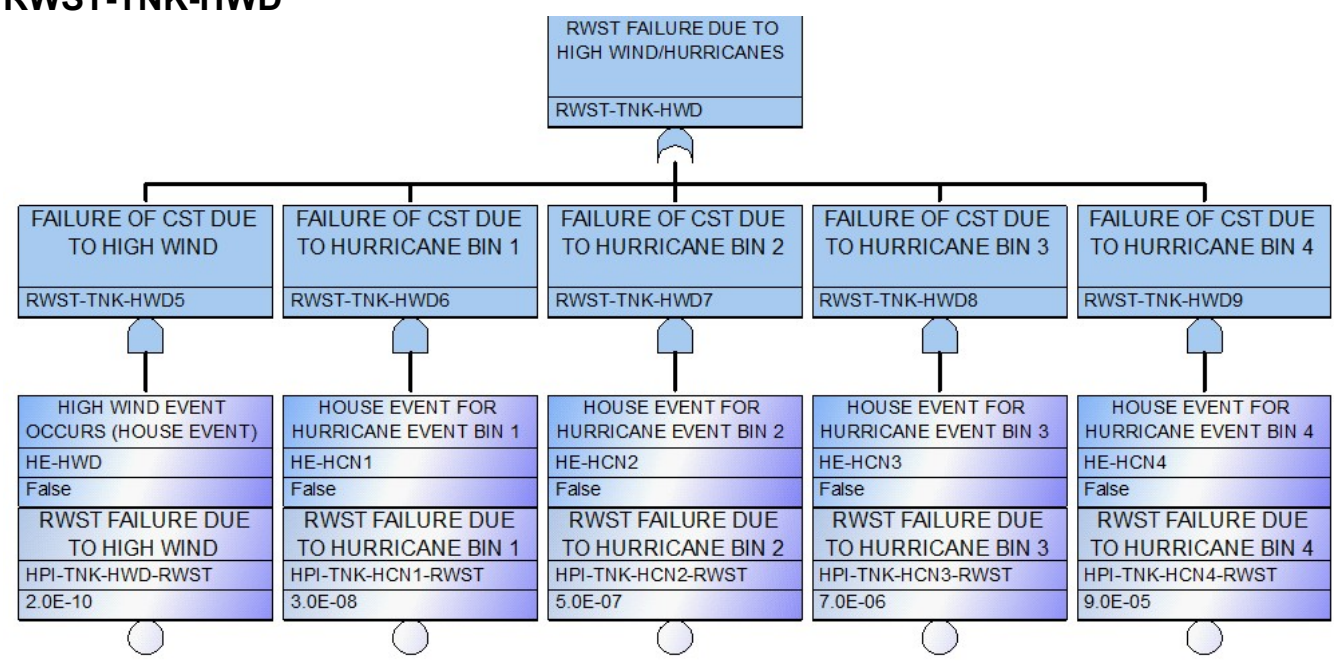

\section{RWST-TNK-TOR}

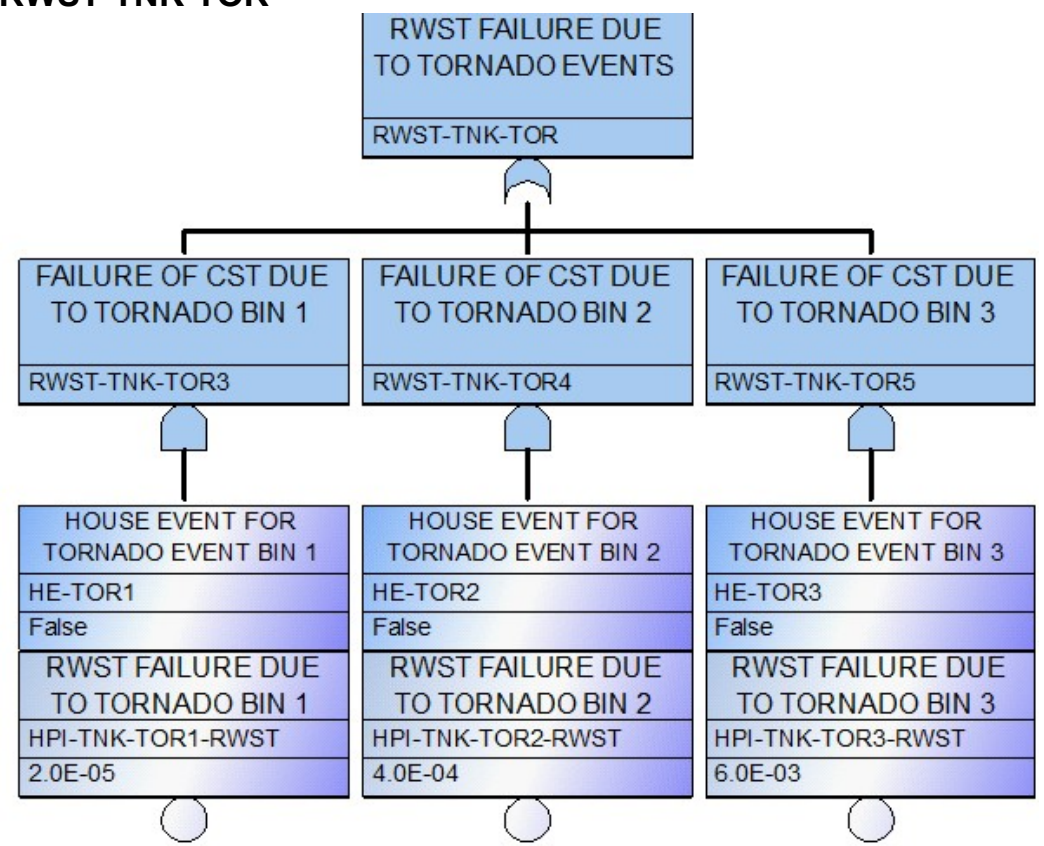

RXVESSEL

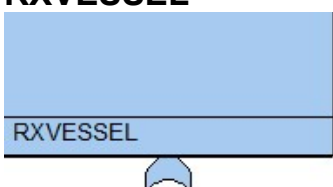

SGI-FT 
Generic Pressurized Water Reactor (PWR)

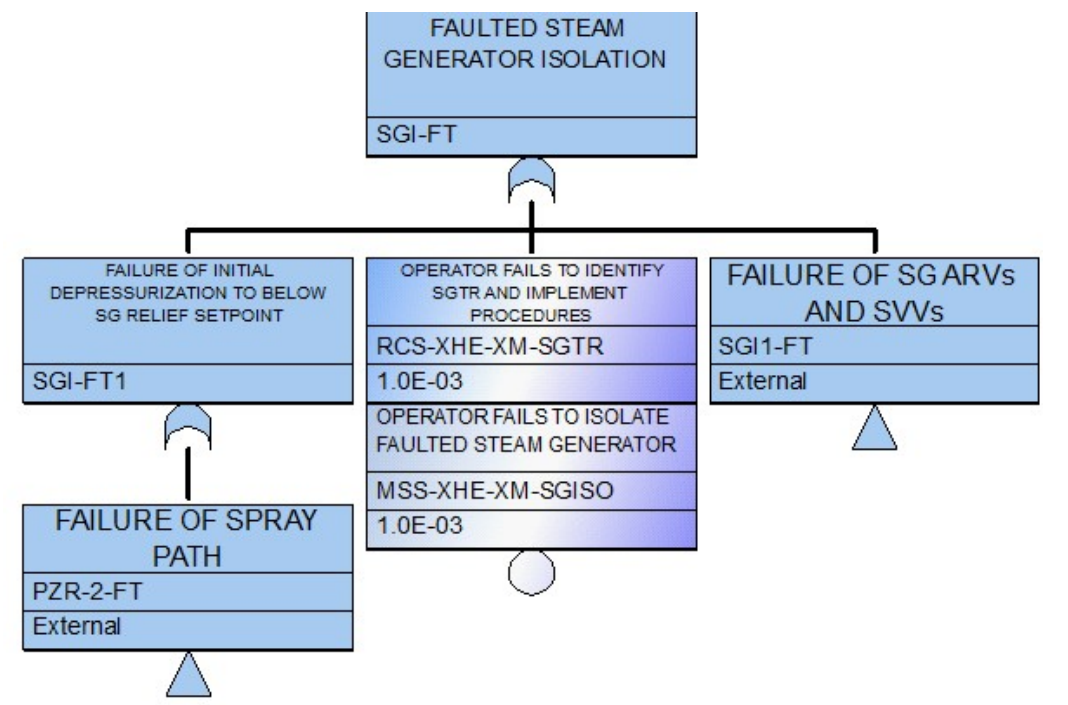

\section{SLOCA-EQ1-FT}

SMALL LOCADUE TO SEISMIC EVENT - BIN 1 SLOCA-EQ1-FT

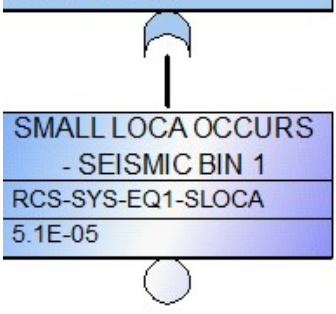

SLOCA-EQ2-FT SMALL LOCADUE TO SEISMIC EVENT - BIN 2

SLOCA-EQ2-FT

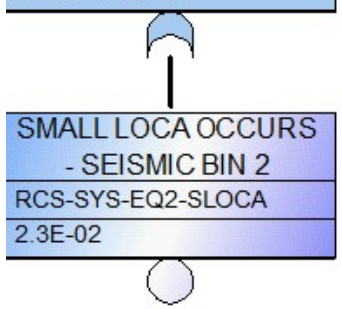

SLOCA-EQ3-FT

SMALL LOCADUE TO SEISMIC EVENT - BIN 3

SLOCA-EQ3-FT

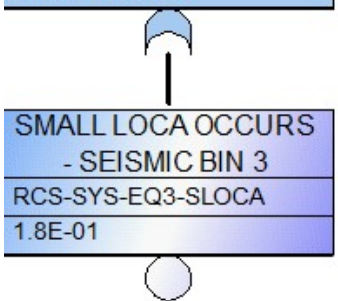

SLOCA-EQ4-FT 
Generic Pressurized Water Reactor (PWR)

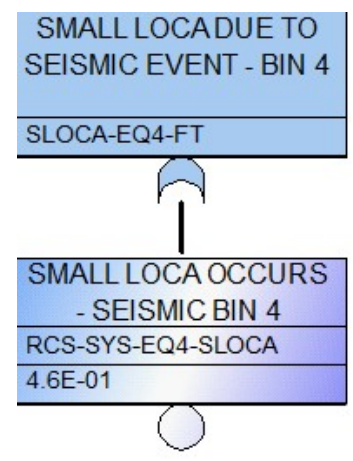

\section{SLOCA-EQ5-FT}

SMALL LOCADUE TO

SEISMIC EVENT - BIN 5

SLOCA-EQ5-FT

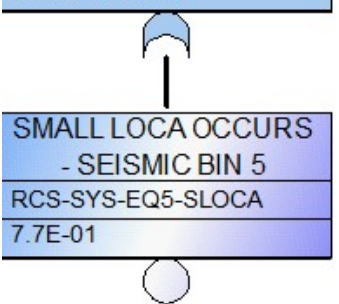

SLOCA-EQ6-FT

SMALL LOCADUE TO SEISMIC EVENT - BIN 6

SLOCA-EQ6-FT

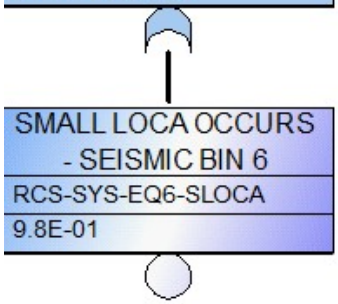

\section{SSC1-FT}

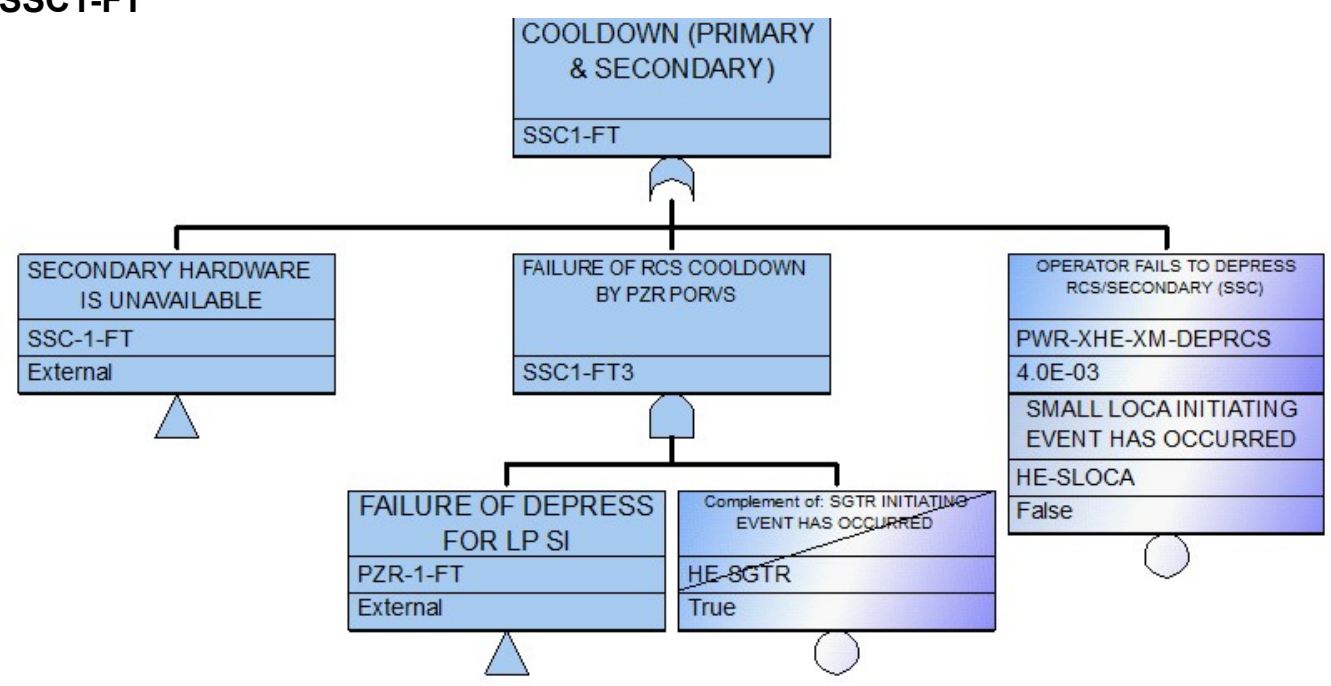

\section{SSC-FT}




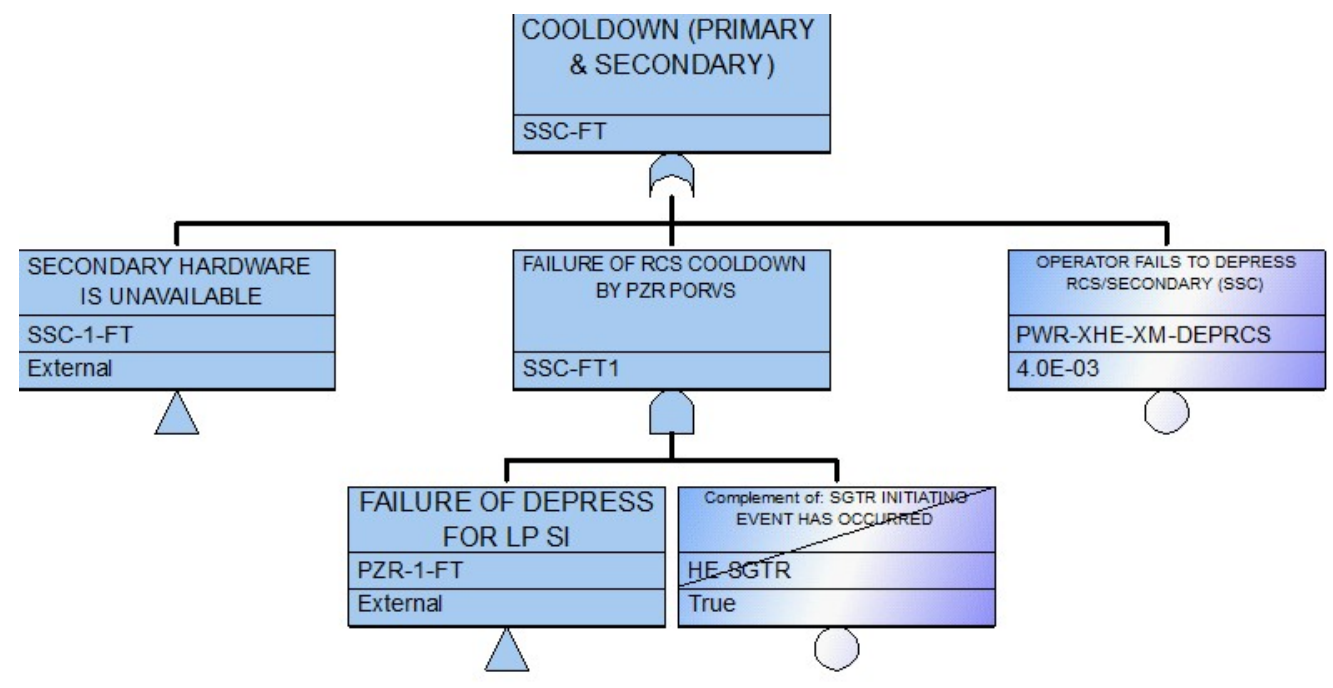

\section{STR-CD-HCN1}

STRUCTURAL FAILURES

DUE TO HURRICANE BIN 1

STR-CD-HCN1

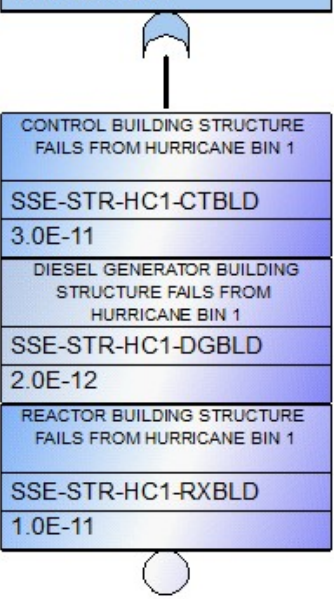

STR-CD-HCN2

STRUCTURAL FAILURES DUE TO HURRICANE BIN 2

STR-CD-HCN2

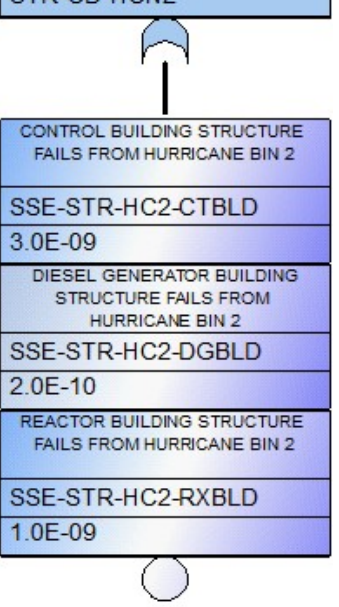

\section{STR-CD-HCN3}


Generic Pressurized Water Reactor (PWR)

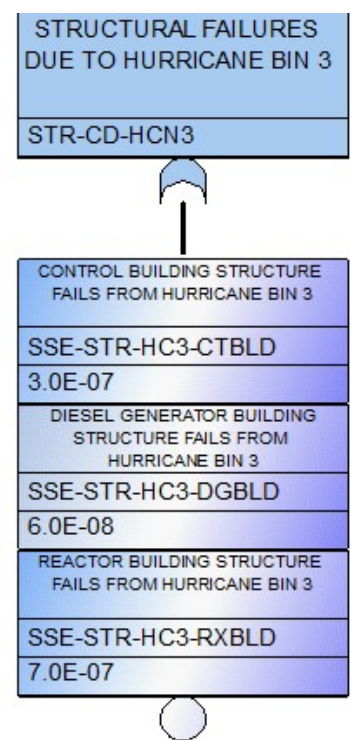

\section{STR-CD-HCN4}

\begin{tabular}{|l|}
\hline STRUCTURAL FAILURES \\
DUE TO HURRICANE BIN 4
\end{tabular}

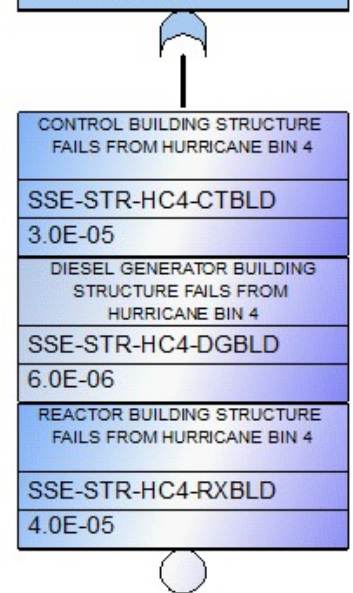

\section{STR-CD-HWD}

STRUCTURAL FAILURES DUE TO HIGH WINDS

STR-CD-HWD

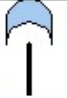

CONTROLBUILDING STRUCTURE FAILS FROM HWD

SSE-STR-HWD-CTBLD

$5.0 \mathrm{E}-14$

DIESEL GENERATOR BUILDING STRUCTURE FAILS FROM HWD

SSE-STR-HWD-DGBLD 2.0E-14

REACTORBUILDING STRUCTURE FAILS FROM HWD

SSE-STR-HWD-RXBLD

$1.0 \mathrm{E}-13$

\section{STR-CD-TOR1}


Generic Pressurized Water Reactor (PWR)

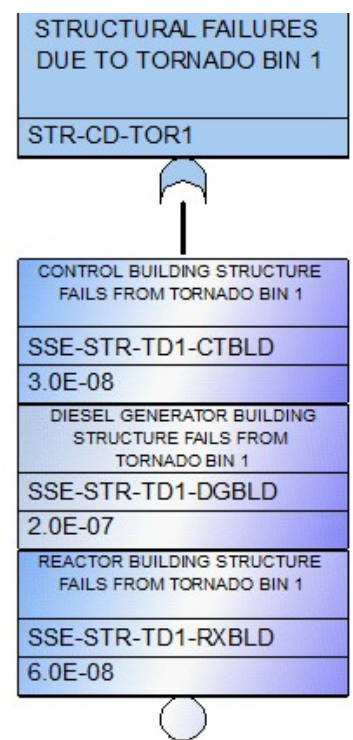

\section{STR-CD-TOR2}

\begin{tabular}{|l|}
\hline STRUCTURAL FAILURES \\
DUE TO TORNADO BIN 2 \\
\hline STR-CD-TOR2 \\
\hline
\end{tabular}

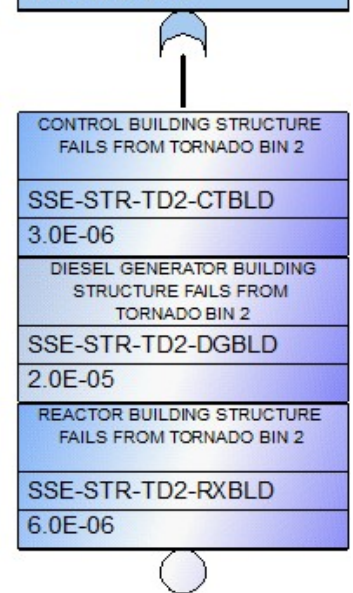

\section{STR-CD-TOR3}

\begin{tabular}{|l|}
\hline STRUCTURAL FAILURES \\
DUE TO TORNADO BIN 3 \\
\hline STR-CD-TOR3 \\
\hline
\end{tabular}

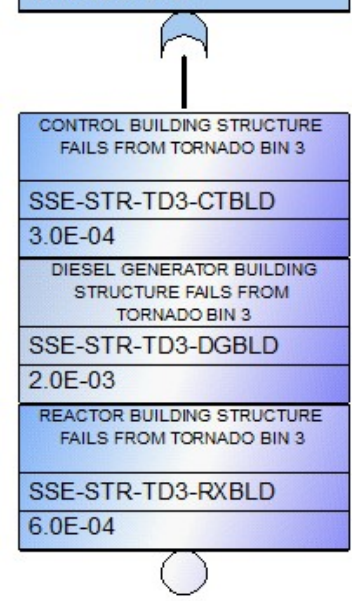

\section{SWS-FT}


Generic Pressurized Water Reactor (PWR)

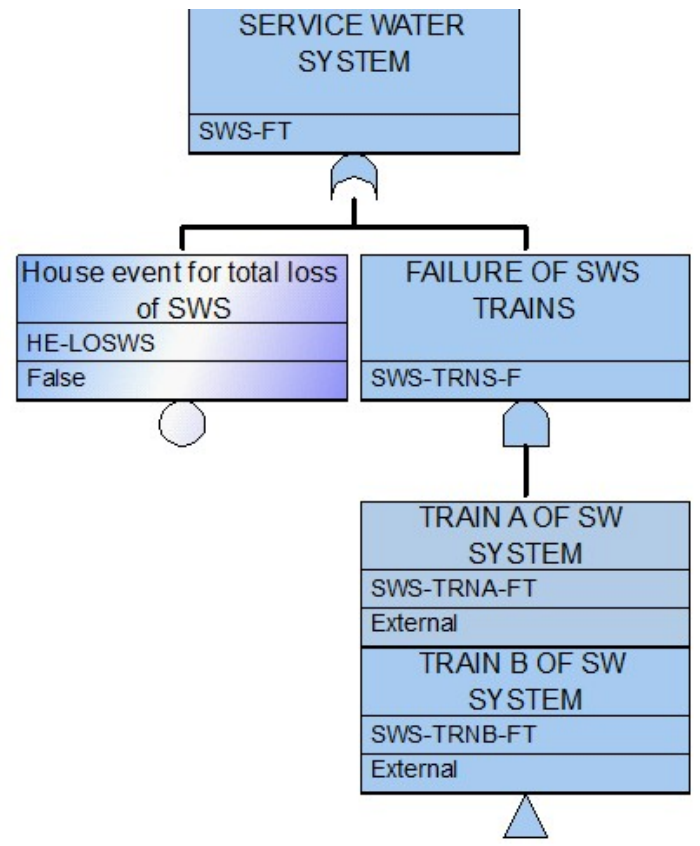

SWS-STR-HWD

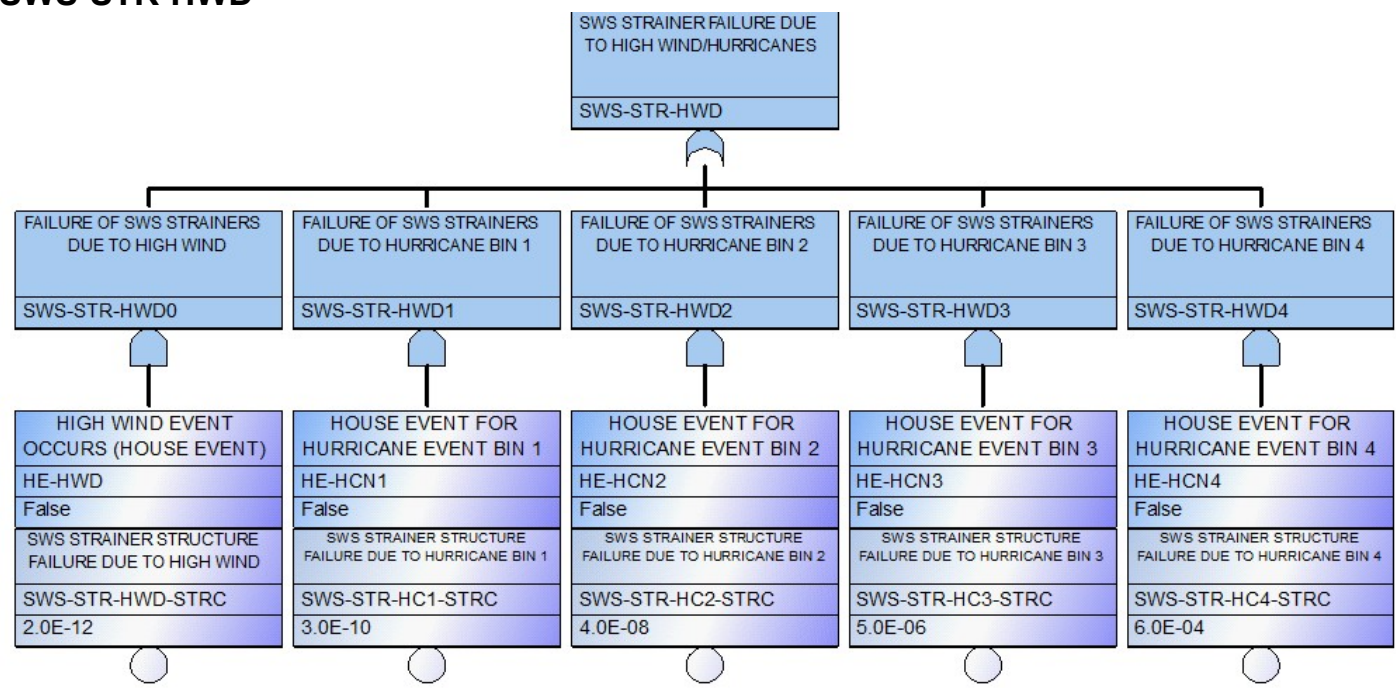

SWS-STR-TOR 


\section{Generic Pressurized Water Reactor (PWR)}

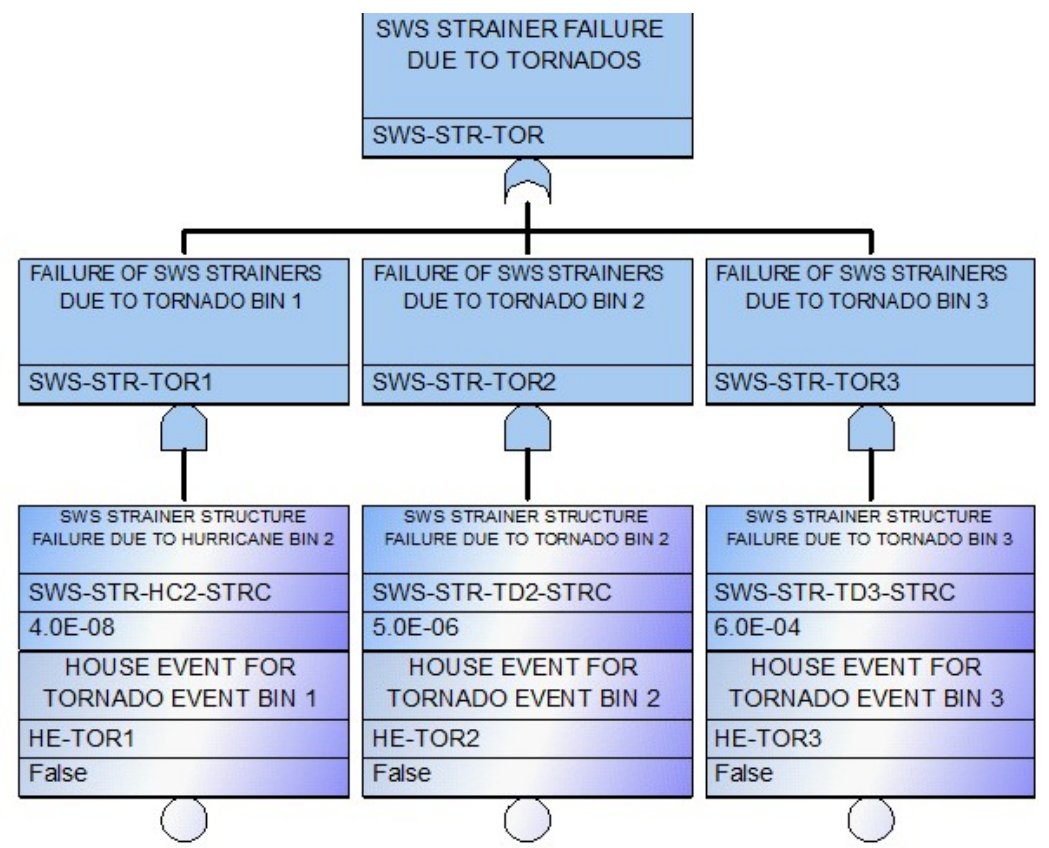

\section{Z-EQK-SEQ-4-03}

\begin{tabular}{|c|} 
SUCCESS EVENTS \\
FOR SEQUENCE 4-03 \\
\hline Z-EQK-SEQ-4-03 \\
\hline
\end{tabular}

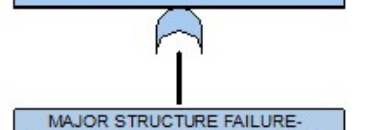

\begin{tabular}{|l|}
\hline $\begin{array}{c}\text { MAJOR STRUCTURE FAILURE- } \\
\text { DIRECT CD FROM SEISMIC EVENT }\end{array}$ \\
\hline CD-EQ4-FT \\
\hline External \\
\hline
\end{tabular}

LLOCADUE TO

SEISMIC EVENT - BIN 4

LLOCA-EQ4-FT

External

REACTOR TRIP

\begin{tabular}{|l|}
\hline RPS-FT \\
\hline External
\end{tabular}

AUXILIARY FEED

WATER SYSTEM

AFW-FT

External

HIGH PRESSURE

INJECTION (CVCS or SIS)

HPI-FT

External

COOLDOWN (PRIMARY

\& SECONDARY)

SSC-FT

External

$\triangle$

\section{Z-EQK-SEQ-4-05}


Generic Pressurized Water Reactor (PWR)

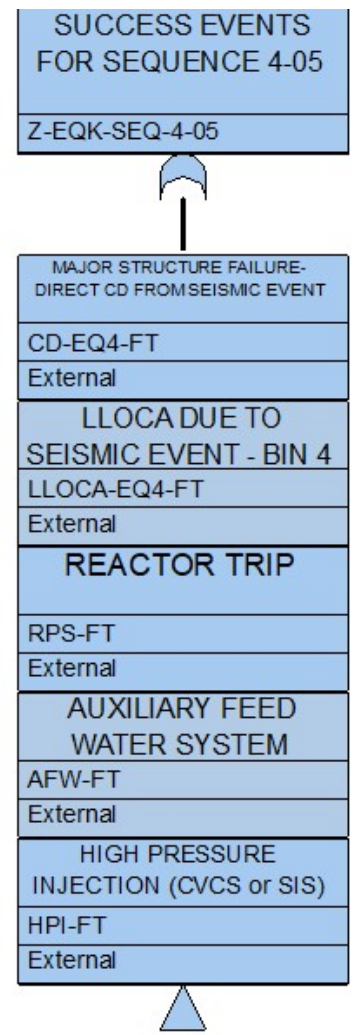

\section{Z-EQK-SEQ-4-08}

SUCCESS EVENTS

FOR SEQUENCE 4-08

Z-EQK-SEQ-4-08

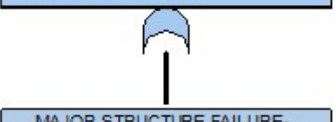

DIRECT CD FROMSEISMIC EVENT

CD-EQ4-FT

External

LLOCADUE TO

SEISMIC EVENT - BIN 4

LLOCA-EQ4-FT

External

REACTOR TRIP

RPS-FT

External

AUXILIARY FEED

WATER SYSTEM

AFW-FT

External

COOLDOWN (PRIMARY

\& SECONDARY)

SSC1-FT

External

LOW PRESSURE

INJECTION SYSTEM

LPI-FT

External

\section{Z-EQK-SEQ-4-09}


Generic Pressurized Water Reactor (PWR)

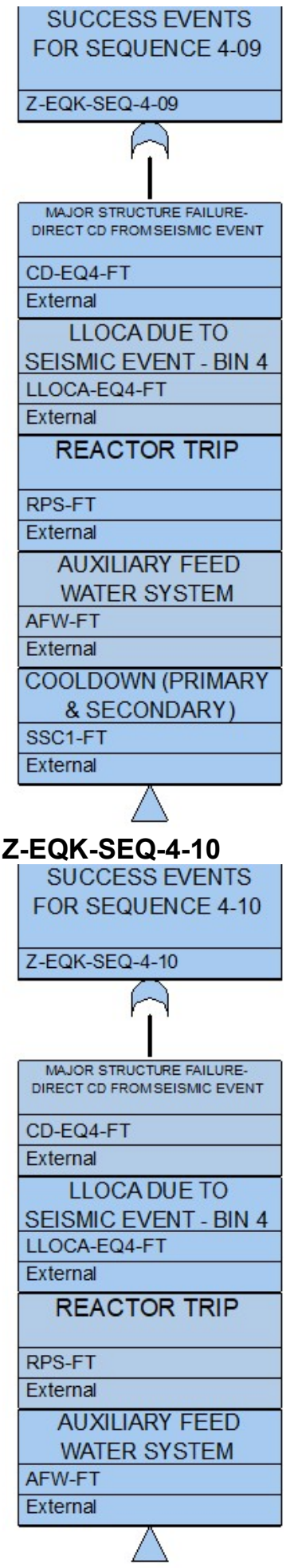

\section{Z-EQK-SEQ-4-12}


Generic Pressurized Water Reactor (PWR)

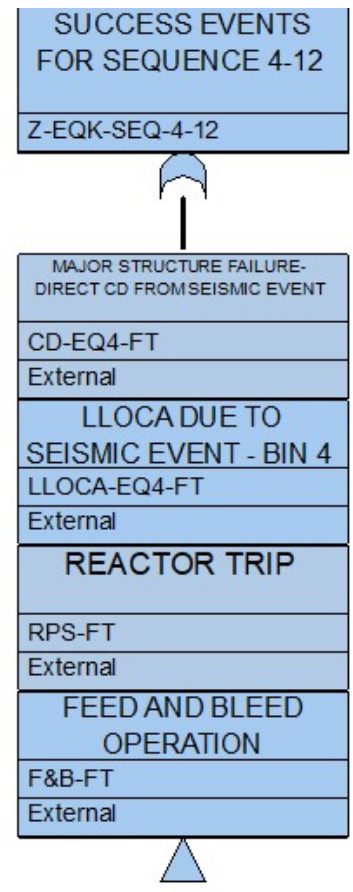

\section{Z-EQK-SEQ-4-13}

\begin{tabular}{|c|}
\hline SUCCESS EVENTS \\
FOR SEQUENCE 4-13 \\
\hline Z-EQK-SEQ-4-13 \\
\hline
\end{tabular}

Z-EQK-SEQ-4-13

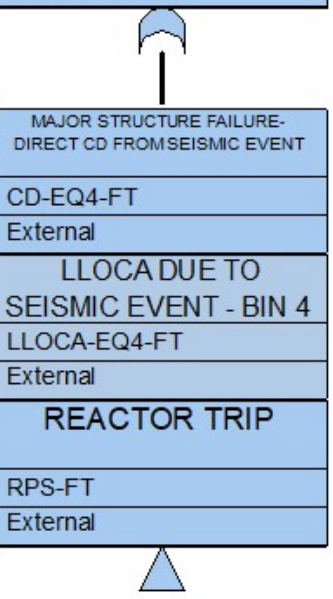

\section{Z-EQK-SEQ-4-14}

\begin{tabular}{|l|} 
SUCCESS EVENTS \\
FOR SEQUENCE 4-14 \\
\hline Z-EQK-SEQ-4-14 \\
\hline MAJOR STRUCTURE FAILURE- \\
DIRECT CD FROM SEISMIC EVENT \\
\hline CD-EQ4-FT \\
\hline EXternal \\
\hline LLOCA DUE TO \\
SEISMIC EVENT - BIN 4 \\
\hline LLOCA-EQ4-FT \\
\hline EXternal \\
\hline
\end{tabular}

\section{Z-EQK-SEQ-5-2}


Generic Pressurized Water Reactor (PWR)

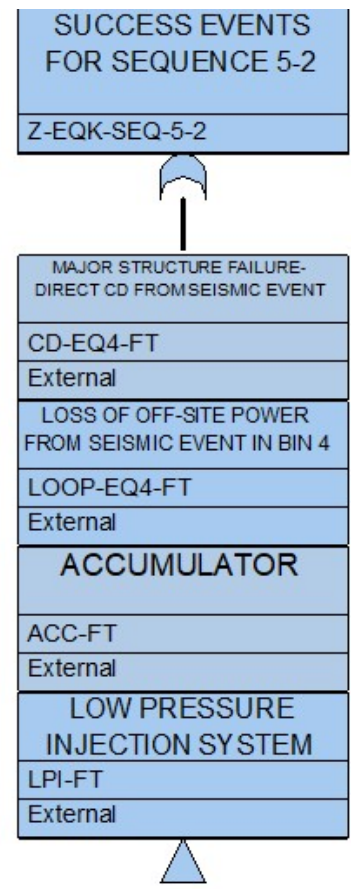

\section{Z-EQK-SEQ-5-3}

\begin{tabular}{|c|}
\hline SUCCESS EVENTS \\
FOR SEQUENCE 5-3 \\
\hline Z-EQK-SEQ-5-3 \\
\hline
\end{tabular}

\begin{tabular}{|l|}
\hline $\begin{array}{c}\text { MASOR STRUCTURE FAILURE- } \\
\text { DIRECT CD FROM SEISMIC EVENT }\end{array}$ \\
\hline CD-EQ4-FT \\
\hline EXternal \\
\hline LOSS OF OFF-SITE POWER \\
FROM SEISMIC EVENT IN BIN 4 \\
\hline LOOP-EQ4-FT \\
\hline EXternal \\
\hline ACCUMULATOR \\
\hline ACC-FT \\
\hline EXternal \\
\hline
\end{tabular}

\section{Z-EQK-SEQ-5-4}

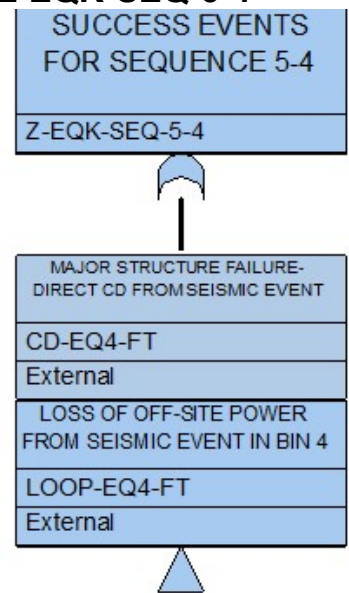

\section{Z-EQK-SEQ-6-2}


Generic Pressurized Water Reactor (PWR)

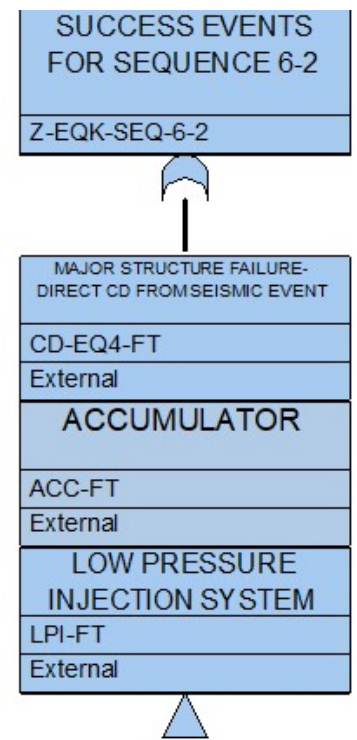

Z-EQK-SEQ-6-3

SUCCESS EVENTS

FOR SEQUENCE 6-3

Z-EQK-SEQ-6-3

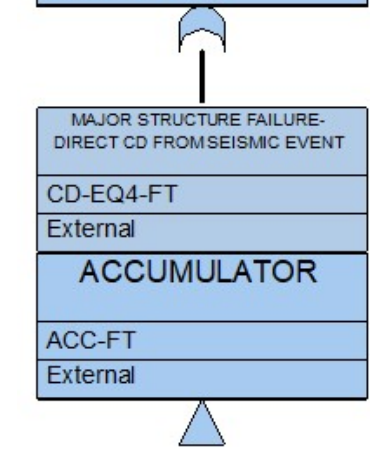

\section{Z-EQK-SEQ-6-4}

SUCCESS EVENTS

FOR SEQUENCE 6-4

Z-EQK-SEQ-6-4

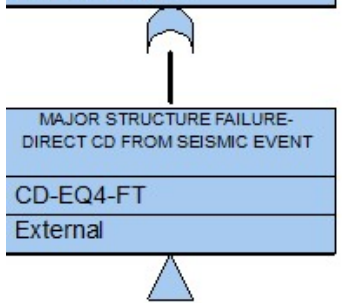

ZV-TOP

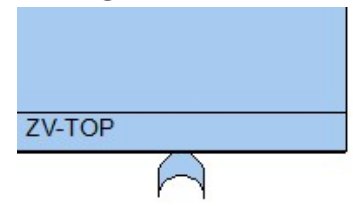




\section{BASIC EVENT INFORMATION}


Basic event notes:

Basic Event Name

\begin{tabular}{ll} 
ACC-CKV-CC-001A \\
ACC-CKV-CC-001B \\
ACC-CKV-CC-001C \\
\hline
\end{tabular}

ACC-MOV-OC-002A http://nrcoe.inel.gov/resultsdb/ParamEstSpar/ February 2017.

U.S. NRC 2015 Parameter Estimation Update

ACC-MOV-OC-002B http://nrcoe.inel.gov/resultsdb/ParamEstSpar/ February2017.

U.S. NRC 2015 Parameter Estimation Update

ACC-MOV-OC-002C http://nrcoe.inel.gov/resultsdb/ParamEstSpar/ February2017.

U.S. NRC 2015 Parameter Estimation Update

ACC-MOV-OC-002D http://nrcoe.inel.gov/resultsdb/ParamEstSpar/ February2017.

U.S. NRC 2015 Parameter Estimation Update

ACC-TNK-FC-ACCA http://nrcoe.inel.gov/resultsdb/ParamEstSpar/ February2017.

U.S. NRC 2015 Parameter Estimation Update

ACC-TNK-FC-ACCB http://nrcoe.inel.gov/resultsdb/ParamEstSpar/ February2017.

U.S. NRC 2015 Parameter Estimation Update

ACC-TNK-FC-ACCC http://nrcoe.inel.gov/resultsdb/ParamEstSpar/ February2017.

U.S. NRC 2015 Parameter Estimation Update

ACC-TNK-FC-ACCD http://nrcoe.inel.gov/resultsdb/ParamEstSpar/ February2017.

U.S. NRC 2015 Parameter Estimation Update

ACC-TNK-LK-ACCA http://nrcoe.inel.gov/resultsdb/ParamEstSpar/ February2017.

U.S. NRC 2015 Parameter Estimation Update

ACC-TNK-LK-ACCB http://nrcoe.inel.gov/resultsdb/ParamEstSpar/ February2017.

U.S. NRC 2015 Parameter Estimation Update

ACC-TNK-LK-ACCC http://nrcoe.inel.gov/resultsdb/ParamEstSpar/ February2017.

U.S. NRC 2015 Parameter Estimation Update

ACC-TNK-LK-ACCD http://nrcoe.inel.gov/resultsdb/ParamEstSpar/ February2017.

U.S. NRC 2015 Parameter Estimation Update

ACC-TNK-RP-ACCA http://nrcoe.inel.gov/resultsdb/ParamEstSpar/ February2017.

U.S. NRC 2015 Parameter Estimation Update

ACC-TNK-RP-ACCB

ACC-TNK-RP-ACCC

http://nrcoe.inel.gov/resultsdb/ParamEstSpar/ February2017.

U.S. NRC 2015 Parameter Estimation Update

http://nrcoe.inel.gov/resultsdb/ParamEstSpar/ February2017.

U.S. NRC 2015 Parameter Estimation Update

ACC-TNK-RP-ACCD http://nrcoe.inel.gov/resultsdb/ParamEstSpar/ February2017.

U.S. NRC 2015 Parameter Estimation Update

ACP-BAC-LP-1A

ACP-BAC-LP-1B

ACP-BAC-LP-480V1A

Model Version: 1.0
http://nrcoe.inel.gov/resultsdb/ParamEstSpar/ February2017.

U.S. NRC 2015 Parameter Estimation Update

http://nrcoe.inel.gov/resultsdb/ParamEstSpar/ February2017.

U.S. NRC 2015 Parameter Estimation Update

http://nrcoe.inel.gov/resultsdb/ParamEstSpar/ February2017. 
Generic Pressurized Water Reactor (PWR)

U.S. NRC 2015 Parameter Estimation Update

\begin{tabular}{|c|c|}
\hline ACP-BAC-LP-480V1B & http://nrcoe.inel.gov/resultsdb/ParamEstSpar/ February2017. \\
\hline ACP-BAC-LP-SWGC & $\begin{array}{l}\text { U.S. NRC } 2015 \text { Parameter Estimation Update } \\
\text { http://nrcoe.inel.gov/resultsdb/ParamEstSpar/ February2017. Section } 5.6\end{array}$ \\
\hline ACP-BAC-LP-SWY101 & $\begin{array}{l}\text { U.S. NRC } 2015 \text { Parameter Estimation Update } \\
\text { http://nrcoe.inel.gov/resultsdb/ParamEstSpar/ February } 2017 .\end{array}$ \\
\hline ACP-BAC-LP-X1A & $\begin{array}{l}\text { U.S. NRC } 2015 \text { Parameter Estimation Update } \\
\text { http://nrcoe.inel.gov/resultsdb/ParamEstSpar/ February } 2017 .\end{array}$ \\
\hline ACP-BAC-LP-XB & $\begin{array}{l}\text { U.S. NRC } 2015 \text { Parameter Estimation Update } \\
\text { http://nrcoe.inel.gov/resultsdb/ParamEstSpar/ February } 2017 .\end{array}$ \\
\hline ACP-CRB-CC-A001 & $\begin{array}{l}\text { U.S. NRC } 2015 \text { Parameter Estimation Update } \\
\text { http://nrcoe.inel.gov/resultsdb/ParamEstSpar/ February } 2017 .\end{array}$ \\
\hline ACP-CRB-CC-A101 & $\begin{array}{l}\text { U.S. NRC } 2015 \text { Parameter Estimation Update } \\
\text { http://nrcoe.inel.gov/resultsdb/ParamEstSpar/ February } 2017 .\end{array}$ \\
\hline ACP-CRB-CC-B001 & $\begin{array}{l}\text { U.S. NRC } 2015 \text { Parameter Estimation Update } \\
\text { http://nrcoe.inel.gov/resultsdb/ParamEstSpar/ February2017. }\end{array}$ \\
\hline ACP-CRB-CC-B101 & $\begin{array}{l}\text { U.S. NRC } 2015 \text { Parameter Estimation Update } \\
\text { http://nrcoe.inel.gov/resultsdb/ParamEstSpar/ February2017. }\end{array}$ \\
\hline ACP-CRB-CO-A103 & $\begin{array}{l}\text { U.S. NRC } 2015 \text { Parameter Estimation Update } \\
\text { http://nrcoe.inel.gov/resultsdb/ParamEstSpar/ February } 2017 .\end{array}$ \\
\hline ACP-CRB-CO-A105 & $\begin{array}{l}\text { U.S. NRC } 2015 \text { Parameter Estimation Update } \\
\text { http://nrcoe.inel.gov/resultsdb/ParamEstSpar/ February } 2017 .\end{array}$ \\
\hline ACP-CRB-CO-A106 & $\begin{array}{l}\text { U.S. NRC } 2015 \text { Parameter Estimation Update } \\
\text { http://nrcoe.inel.gov/resultsdb/ParamEstSpar/ February } 2017 .\end{array}$ \\
\hline ACP-CRB-CO-B105 & $\begin{array}{l}\text { U.S. NRC } 2015 \text { Parameter Estimation Update } \\
\text { http://nrcoe.inel.gov/resultsdb/ParamEstSpar/ February2017. }\end{array}$ \\
\hline ACP-CRB-CO-B106 & $\begin{array}{l}\text { U.S. NRC } 2015 \text { Parameter Estimation Update } \\
\text { http://nrcoe.inel.gov/resultsdb/ParamEstSpar/ February } 2017 .\end{array}$ \\
\hline ACP-CRB-OC-B103 & $\begin{array}{l}\text { U.S. NRC } 2015 \text { Parameter Estimation Update } \\
\text { http://nrcoe.inel.gov/resultsdb/ParamEstSpar/ February } 2017 .\end{array}$ \\
\hline ACP-CRB-OO-A102 & $\begin{array}{l}\text { U.S. NRC } 2015 \text { Parameter Estimation Update } \\
\text { http://nrcoe.inel.gov/resultsdb/ParamEstSpar/ February } 2017 .\end{array}$ \\
\hline ACP-CRB-OO-B102 & $\begin{array}{l}\text { U.S. NRC } 2015 \text { Parameter Estimation Update } \\
\text { http://nrcoe.inel.gov/resultsdb/ParamEstSpar/ February } 2017 .\end{array}$ \\
\hline ACP-CRB-OO-SWGC & $\begin{array}{l}\text { U.S. NRC } 2015 \text { Parameter Estimation Update } \\
\text { http://nrcoe.inel.gov/resultsdb/ParamEstSpar/ February2017. Section } 5.4\end{array}$ \\
\hline ACP-TFM-FC-480V1A & $\begin{array}{l}\text { U.S. NRC } 2015 \text { Parameter Estimation Update } \\
\text { http://nrcoe.inel.gov/resultsdb/ParamEstSpar/ February2017. }\end{array}$ \\
\hline ACP-TFM-FC-480V1B & $\begin{array}{l}\text { U.S. NRC } 2015 \text { Parameter Estimation Update } \\
\text { http://nrcoe.inel.gov/resultsdb/ParamEstSpar/ February2017. }\end{array}$ \\
\hline ACP-TFM-FC-X1A & $\begin{array}{l}\text { U.S. NRC } 2015 \text { Parameter Estimation Update } \\
\text { http://nrcoe.inel.gov/resultsdb/ParamEstSpar/ February } 2017 .\end{array}$ \\
\hline ACP-TFM-FC-XB & $\begin{array}{l}\text { U.S. NRC } 2015 \text { Parameter Estimation Update } \\
\text { http://nrcoe.inel.gov/resultsdb/ParamEstSpar/ February } 2017 .\end{array}$ \\
\hline AFW-AOV-OC-TDPSGA & $\begin{array}{l}\text { U.S. NRC } 2015 \text { Parameter Estimation Update } \\
\text { http://nrcoe.inel.gov/resultsdb/ParamEstSpar/ February2017. }\end{array}$ \\
\hline AFW-AOV-OC-TDPSGB & $\begin{array}{l}\text { U.S. NRC } 2015 \text { Parameter Estimation Update } \\
\text { http://nrcoe.inel.gov/resultsdb/ParamEstSpar/ February } 2017 .\end{array}$ \\
\hline AFW-AOV-OC-TDPSGC & $\begin{array}{l}\text { U.S. NRC } 2015 \text { Parameter Estimation Update } \\
\text { http://nrcoe.inel.gov/resultsdb/ParamEstSpar/ February } 2017 .\end{array}$ \\
\hline
\end{tabular}


Generic Pressurized Water Reactor (PWR)

U.S. NRC 2015 Parameter Estimation Update

AFW-AOV-OC-TDPSGD http://nrcoe.inel.gov/resultsdb/ParamEstSpar/ February2017.

U.S. NRC 2015 Parameter Estimation Update

AFW-CKV-CC-001A http://nrcoe.inel.gov/resultsdb/ParamEstSpar/ February2017.

U.S. NRC 2015 Parameter Estimation Update

AFW-CKV-CC-001B http://nrcoe.inel.gov/resultsdb/ParamEstSpar/ February2017.

U.S. NRC 2015 Parameter Estimation Update

AFW-CKV-CC-001C http://nrcoe.inel.gov/resultsdb/ParamEstSpar/ February2017.

U.S. NRC 2015 Parameter Estimation Update

AFW-CKV-CC-001D http://nrcoe.inel.gov/resultsdb/ParamEstSpar/ February2017.

U.S. NRC 2015 Parameter Estimation Update

AFW-CKV-CC-002A http://nrcoe.inel.gov/resultsdb/ParamEstSpar/ February2017.

U.S. NRC 2015 Parameter Estimation Update

AFW-CKV-CC-002B http://nrcoe.inel.gov/resultsdb/ParamEstSpar/ February2017.

U.S. NRC 2015 Parameter Estimation Update

AFW-CKV-CC-002C http://nrcoe.inel.gov/resultsdb/ParamEstSpar/ February2017.

U.S. NRC 2015 Parameter Estimation Update

AFW-CKV-CC-002D http://nrcoe.inel.gov/resultsdb/ParamEstSpar/ February2017.

U.S. NRC 2015 Parameter Estimation Update

AFW-CKV-CC-003A http://nrcoe.inel.gov/resultsdb/ParamEstSpar/ February2017.

U.S. NRC 2015 Parameter Estimation Update

AFW-CKV-CC-003B http://nrcoe.inel.gov/resultsdb/ParamEstSpar/ February2017.

U.S. NRC 2015 Parameter Estimation Update

AFW-CKV-CC-003C http://nrcoe.inel.gov/resultsdb/ParamEstSpar/ February2017.

U.S. NRC 2015 Parameter Estimation Update

AFW-CKV-CC-003D http://nrcoe.inel.gov/resultsdb/ParamEstSpar/ February2017.

U.S. NRC 2015 Parameter Estimation Update

AFW-CKV-CC-MPA01 http://nrcoe.inel.gov/resultsdb/ParamEstSpar/ February2017.

U.S. NRC 2015 Parameter Estimation Update

AFW-CKV-CC-MPA02 http://nrcoe.inel.gov/resultsdb/ParamEstSpar/ February2017.

U.S. NRC 2015 Parameter Estimation Update

AFW-CKV-CC-MPB01 http://nrcoe.inel.gov/resultsdb/ParamEstSpar/ February2017.

U.S. NRC 2015 Parameter Estimation Update

AFW-CKV-CC-MPB02 http://nrcoe.inel.gov/resultsdb/ParamEstSpar/ February2017.

U.S. NRC 2015 Parameter Estimation Update

AFW-CKV-CC-TP01 http://nrcoe.inel.gov/resultsdb/ParamEstSpar/ February2017.

U.S. NRC 2015 Parameter Estimation Update

AFW-CKV-CC-TP02 http://nrcoe.inel.gov/resultsdb/ParamEstSpar/ February2017.

AFW-CKV-CF-001 Common cause failure event using the generic alpha factors.

AFW-CKV-CF-MPTP1 Common cause failure event using the generic alpha factors.

AFW-CKV-CF-MPTP2 Common cause failure event using the generic alpha factors.

U.S. NRC 2015 Parameter Estimation Update

AFW-FCV-OC-MDPA http://nrcoe.inel.gov/resultsdb/ParamEstSpar/ February2017.

U.S. NRC 2015 Parameter Estimation Update

AFW-FCV-OC-MDPB http://nrcoe.inel.gov/resultsdb/ParamEstSpar/ February2017.

U.S. NRC 2015 Parameter Estimation Update

AFW-FCV-OC-TDP http://nrcoe.inel.gov/resultsdb/ParamEstSpar/ February2017.

AFW-MDP-CF-FR Common cause failure event using the generic alpha factors.

AFW-MDP-CF-FS Common cause failure event using the generic alpha factors. 
U.S. NRC 2015 Parameter Estimation Update

AFW-MDP-FR-A

http://nrcoe.inel.gov/resultsdb/ParamEstSpar/ February2017.

U.S. NRC 2015 Parameter Estimation Update

AFW-MDP-FR-B http://nrcoe.inel.gov/resultsdb/ParamEstSpar/ February2017.

U.S. NRC 2015 Parameter Estimation Update

AFW-MDP-FS-A http://nrcoe.inel.gov/resultsdb/ParamEstSpar/ February2017.

U.S. NRC 2015 Parameter Estimation Update

AFW-MDP-FS-B http://nrcoe.inel.gov/resultsdb/ParamEstSpar/ February2017.

U.S. NRC 2015 Parameter Estimation Update

AFW-MOV-OC-004A http://nrcoe.inel.gov/resultsdb/ParamEstSpar/ February2017.

U.S. NRC 2015 Parameter Estimation Update

AFW-MOV-OC-004B http://nrcoe.inel.gov/resultsdb/ParamEstSpar/ February2017.

U.S. NRC 2015 Parameter Estimation Update

AFW-MOV-OC-004C http://nrcoe.inel.gov/resultsdb/ParamEstSpar/ February2017.

U.S. NRC 2015 Parameter Estimation Update

AFW-MOV-OC-004D http://nrcoe.inel.gov/resultsdb/ParamEstSpar/ February2017.

AFW-PMP-CF-FR Common cause failure event using the generic alpha factors.

U.S. NRC 2015 Parameter Estimation Update

AFW-TDP-FR-TDP http://nrcoe.inel.gov/resultsdb/ParamEstSpar/ February2017.

U.S. NRC 2015 Parameter Estimation Update

AFW-TDP-FS-TDP http://nrcoe.inel.gov/resultsdb/ParamEstSpar/ February2017.

U.S. NRC 2015 Parameter Estimation Update

AFW-TNK-FC-CST http://nrcoe.inel.gov/resultsdb/ParamEstSpar/ February2017.

U.S. NRC 2015 Parameter Estimation Update

CCW-AOV-OC-HTXA http://nrcoe.inel.gov/resultsdb/ParamEstSpar/ February2017.

U.S. NRC 2015 Parameter Estimation Update

CCW-AOV-OC-HTXB http://nrcoe.inel.gov/resultsdb/ParamEstSpar/ February2017.

U.S. NRC 2015 Parameter Estimation Update

CCW-AOV-OC-HTXDISA http://nrcoe.inel.gov/resultsdb/ParamEstSpar/ February2017.

U.S. NRC 2015 Parameter Estimation Update

CCW-AOV-OC-HTXDISB http://nrcoe.inel.gov/resultsdb/ParamEstSpar/ February2017.

U.S. NRC 2015 Parameter Estimation Update

CCW-CKV-CC-002B http://nrcoe.inel.gov/resultsdb/ParamEstSpar/ February2017.

CCW-HTX-CF-PG Common cause failure event using the generic alpha factors.

U.S. NRC 2015 Parameter Estimation Update

CCW-HTX-LK-1A http://nrcoe.inel.gov/resultsdb/ParamEstSpar/ February2017.

U.S. NRC 2015 Parameter Estimation Update

CCW-HTX-LK-1B http://nrcoe.inel.gov/resultsdb/ParamEstSpar/ February2017.

U.S. NRC 2015 Parameter Estimation Update

CCW-HTX-PG-1A http://nrcoe.inel.gov/resultsdb/ParamEstSpar/ February2017.

U.S. NRC 2015 Parameter Estimation Update

CCW-HTX-PG-1B http://nrcoe.inel.gov/resultsdb/ParamEstSpar/ February2017.

U.S. NRC 2015 Parameter Estimation Update

CCW-HTX-RP-1A http://nrcoe.inel.gov/resultsdb/ParamEstSpar/ February2017.

U.S. NRC 2015 Parameter Estimation Update

CCW-HTX-RP-1B

CCW-MDP-CF-FTR

http://nrcoe.inel.gov/resultsdb/ParamEstSpar/ February2017.

Common cause failure event using the generic alpha factors.

U.S. NRC 2015 Parameter Estimation Update

CCW-MDP-FR-1A

http://nrcoe.inel.gov/resultsdb/ParamEstSpar/ February2017. 
U.S. NRC 2015 Parameter Estimation Update

CCW-MDP-FR-1B

CCW-MDP-FR-1C

CCW-MDP-FS-1A

CCW-MDP-FS-1B

CCW-MDP-FS-1C

CCW-MOV-OC-001A

CCW-MOV-OC-001B

CCW-MOV-OC-001C_A
CCW-MOV-OC-001C_B

CCW-MOV-OC-002A

CCW-MOV-OC-002B

CCW-MOV-OC-002C_A

CCW-MOV-OC-002C_B

CCW-TNK-FC-SURGE

CSS-SMP-PG-A

CSS-SMP-PG-B

CVC-CKV-CC-004A

CVC-CKV-CC-004B

CVC-MDP-FR-001A

CVC-MDP-FR-001B

CVC-MDP-FS-001A

CVC-MDP-FS-001B

CVC-MDP-TM-001A

CVC-MDP-TM-001B

CVC-MOV-CC-TRNA
http://nrcoe.inel.gov/resultsdb/ParamEstSpar/ February2017.

U.S. NRC 2015 Parameter Estimation Update

http://nrcoe.inel.gov/resultsdb/ParamEstSpar/ February2017.

U.S. NRC 2015 Parameter Estimation Update

http://nrcoe.inel.gov/resultsdb/ParamEstSpar/ February2017.

U.S. NRC 2015 Parameter Estimation Update

http://nrcoe.inel.gov/resultsdb/ParamEstSpar/ February2017.

U.S. NRC 2015 Parameter Estimation Update

http://nrcoe.inel.gov/resultsdb/ParamEstSpar/ February2017.

U.S. NRC 2015 Parameter Estimation Update

http://nrcoe.inel.gov/resultsdb/ParamEstSpar/ February2017.

U.S. NRC 2015 Parameter Estimation Update

http://nrcoe.inel.gov/resultsdb/ParamEstSpar/ February2017.

U.S. NRC 2015 Parameter Estimation Update

http://nrcoe.inel.gov/resultsdb/ParamEstSpar/ February2017.

U.S. NRC 2015 Parameter Estimation Update

http://nrcoe.inel.gov/resultsdb/ParamEstSpar/ February2017.

U.S. NRC 2015 Parameter Estimation Update

http://nrcoe.inel.gov/resultsdb/ParamEstSpar/ February2017.

U.S. NRC 2015 Parameter Estimation Update

http://nrcoe.inel.gov/resultsdb/ParamEstSpar/ February2017.

U.S. NRC 2015 Parameter Estimation Update

http://nrcoe.inel.gov/resultsdb/ParamEstSpar/ February2017.

U.S. NRC 2015 Parameter Estimation Update

http://nrcoe.inel.gov/resultsdb/ParamEstSpar/ February2017.

U.S. NRC 2015 Parameter Estimation Update

http://nrcoe.inel.gov/resultsdb/ParamEstSpar/ February2017.

U.S. NRC 2015 Parameter Estimation Update

http://nrcoe.inel.gov/resultsdb/ParamEstSpar/ February2017.

U.S. NRC 2015 Parameter Estimation Update

http://nrcoe.inel.gov/resultsdb/ParamEstSpar/ February2017.

U.S. NRC 2015 Parameter Estimation Update

http://nrcoe.inel.gov/resultsdb/ParamEstSpar/ February2017.

U.S. NRC 2015 Parameter Estimation Update

http://nrcoe.inel.gov/resultsdb/ParamEstSpar/ February2017.

U.S. NRC 2015 Parameter Estimation Update

http://nrcoe.inel.gov/resultsdb/ParamEstSpar/ February2017.

U.S. NRC 2015 Parameter Estimation Update

http://nrcoe.inel.gov/resultsdb/ParamEstSpar/ February2017.

U.S. NRC 2015 Parameter Estimation Update

http://nrcoe.inel.gov/resultsdb/ParamEstSpar/ February2017.

U.S. NRC 2015 Parameter Estimation Update

http://nrcoe.inel.gov/resultsdb/ParamEstSpar/ February2017.

2015 Update to the Parameter Estimation Component Unavailability Data Sheets Section 1

Table 1-1

2015 Update to the Parameter Estimation Component Unavailability Data Sheets Section 1

Table 1-1

U.S. NRC 2015 Parameter Estimation Update

http://nrcoe.inel.gov/resultsdb/ParamEstSpar/ February2017. 
Generic Pressurized Water Reactor (PWR)

U.S. NRC 2015 Parameter Estimation Update

\begin{tabular}{|c|c|}
\hline CVC-MOV-CC-TRNB & http://nrcoe.inel.gov/resultsdb/ParamEstSpar/ February2017. \\
\hline CVC-MOV-OC-001 & $\begin{array}{l}\text { U.S. NRC } 2015 \text { Parameter Estimation Update } \\
\text { http://nrcoe.inel.gov/resultsdb/ParamEstSpar/ February } 2017 .\end{array}$ \\
\hline CVC-MOV-OC-002 & $\begin{array}{l}\text { U.S. NRC } 2015 \text { Parameter Estimation Update } \\
\text { http://nrcoe.inel.gov/resultsdb/ParamEstSpar/ February } 2017 .\end{array}$ \\
\hline DCP-BAT-LP-A01 & $\begin{array}{l}\text { U.S. NRC } 2015 \text { Parameter Estimation Update } \\
\text { http://nrcoe.inel.gov/resultsdb/ParamEstSpar/ February2017. }\end{array}$ \\
\hline DCP-BAT-LP-B01 & $\begin{array}{l}\text { U.S. NRC } 2015 \text { Parameter Estimation Update } \\
\text { http://nrcoe.inel.gov/resultsdb/ParamEstSpar/ February2017. }\end{array}$ \\
\hline DCP-BAT-LP-SCBAT & $\begin{array}{l}\text { U.S. NRC } 2015 \text { Parameter Estimation Update } \\
\text { http://nrcoe.inel.gov/resultsdb/ParamEstSpar/ February2017. Section } 5.2\end{array}$ \\
\hline DCP-BCH-FC-A01 & $\begin{array}{l}\text { U.S. NRC } 2015 \text { Parameter Estimation Update } \\
\text { http://nrcoe.inel.gov/resultsdb/ParamEstSpar/ February2017. }\end{array}$ \\
\hline DCP-BCH-FC-B01 & $\begin{array}{l}\text { U.S. NRC } 2015 \text { Parameter Estimation Update } \\
\text { http://nrcoe.inel.gov/resultsdb/ParamEstSpar/ February2017. }\end{array}$ \\
\hline DCP-BDC-LP-1A & $\begin{array}{l}\text { U.S. NRC } 2015 \text { Parameter Estimation Update } \\
\text { http://nrcoe.inel.gov/resultsdb/ParamEstSpar/ February2017. Section } 5.6\end{array}$ \\
\hline DCP-BDC-LP-1B & $\begin{array}{l}\text { U.S. NRC } 2015 \text { Parameter Estimation Update } \\
\text { http://nrcoe.inel.gov/resultsdb/ParamEstSpar/ February2017. Section } 5.6\end{array}$ \\
\hline DCP-BDC-LP-SWGC & $\begin{array}{l}\text { U.S. NRC } 2015 \text { Parameter Estimation Update } \\
\text { http://nrcoe.inel.gov/resultsdb/ParamEstSpar/ February2017. Section } 5.6\end{array}$ \\
\hline EPS-CRB-OO-A104 & $\begin{array}{l}\text { U.S. NRC } 2015 \text { Parameter Estimation Update } \\
\text { http://nrcoe.inel.gov/resultsdb/ParamEstSpar/ February2017. }\end{array}$ \\
\hline EPS-CRB-OO-B104 & $\begin{array}{l}\text { U.S. NRC } 2015 \text { Parameter Estimation Update } \\
\text { http://nrcoe.inel.gov/resultsdb/ParamEstSpar/ February2017. }\end{array}$ \\
\hline EPS-DGN-FR-DGNA & $\begin{array}{l}\text { U.S. NRC } 2015 \text { Parameter Estimation Update } \\
\text { http://nrcoe.inel.gov/resultsdb/ParamEstSpar/ February2017. }\end{array}$ \\
\hline EPS-DGN-FR-DGNB & $\begin{array}{l}\text { U.S. NRC } 2015 \text { Parameter Estimation Update } \\
\text { http://nrcoe.inel.gov/resultsdb/ParamEstSpar/ February2017. }\end{array}$ \\
\hline EPS-DGN-FR-SBO & $\begin{array}{l}\text { U.S. NRC } 2015 \text { Parameter Estimation Update } \\
\text { http://nrcoe.inel.gov/resultsdb/ParamEstSpar/ February2017. Section } 3.1\end{array}$ \\
\hline EPS-DGN-FS-DGNA & $\begin{array}{l}\text { U.S. NRC } 2015 \text { Parameter Estimation Update } \\
\text { http://nrcoe.inel.gov/resultsdb/ParamEstSpar/ February2017. }\end{array}$ \\
\hline EPS-DGN-FS-DGNB & $\begin{array}{l}\text { U.S. NRC } 2015 \text { Parameter Estimation Update } \\
\text { http://nrcoe.inel.gov/resultsdb/ParamEstSpar/ February2017. }\end{array}$ \\
\hline EPS-DGN-FS-SBO & $\begin{array}{l}\text { U.S. NRC } 2015 \text { Parameter Estimation Update } \\
\text { http://nrcoe.inel.gov/resultsdb/ParamEstSpar/ February } 2017 .\end{array}$ \\
\hline EPS-DGN-LR-DGNA & $\begin{array}{l}\text { U.S. NRC } 2015 \text { Parameter Estimation Update } \\
\text { http://nrcoe.inel.gov/resultsdb/ParamEstSpar/ February2017. }\end{array}$ \\
\hline EPS-DGN-LR-DGNB & $\begin{array}{l}\text { U.S. NRC } 2015 \text { Parameter Estimation Update } \\
\text { http://nrcoe.inel.gov/resultsdb/ParamEstSpar/ February } 2017 .\end{array}$ \\
\hline EPS-DGN-LR-SBO & $\begin{array}{l}\text { U.S. NRC } 2015 \text { Parameter Estimation Update } \\
\text { http://nrcoe.inel.gov/resultsdb/ParamEstSpar/ February2017. Section } 3.1\end{array}$ \\
\hline EPS-FAN-FR-SBOHVAC & $\begin{array}{l}\text { U.S. NRC } 2015 \text { Parameter Estimation Update } \\
\text { http://nrcoe.inel.gov/resultsdb/ParamEstSpar/ February2017. }\end{array}$ \\
\hline EPS-FAN-FS-SBOHVAC & $\begin{array}{l}\text { U.S. NRC } 2015 \text { Parameter Estimation Update } \\
\text { http://nrcoe.inel.gov/resultsdb/ParamEstSpar/ February2017. }\end{array}$ \\
\hline EPS-FAN-LR-SBOHVAC & $\begin{array}{l}\text { U.S. NRC } 2015 \text { Parameter Estimation Update } \\
\text { http://nrcoe.inel.gov/resultsdb/ParamEstSpar/ February2017. }\end{array}$ \\
\hline
\end{tabular}


U.S. NRC 2015 Parameter Estimation Update

\begin{tabular}{|c|c|}
\hline EPS-SEQ-FC-DGNA & http://nrcoe.inel.gov/resultsdb/ParamEstSpar/ February2017. Section 5.9 \\
\hline EPS-SEQ-FC-DGNB & $\begin{array}{l}\text { U.S. NRC } 2015 \text { Parameter Estimation Update } \\
\text { http://nrcoe.inel.gov/resultsdb/ParamEstSpar/ February2017. Section } 5.9\end{array}$ \\
\hline FLX-DGN-FR-DG1 & $\begin{array}{l}\text { U.S. NRC } 2015 \text { Parameter Estimation Update } \\
\text { http://nrcoe.inel.gov/resultsdb/ParamEstSpar/ February2017. Section } 3.1\end{array}$ \\
\hline FLX-DGN-FR-DG2 & $\begin{array}{l}\text { U.S. NRC } 2015 \text { Parameter Estimation Update } \\
\text { http://nrcoe.inel.gov/resultsdb/ParamEstSpar/ February2017. Section } 3.1\end{array}$ \\
\hline FLX-DGN-FS-DG1 & $\begin{array}{l}\text { U.S. NRC } 2015 \text { Parameter Estimation Update } \\
\text { http://nrcoe.inel.gov/resultsdb/ParamEstSpar/ February2017. Section } 3.1\end{array}$ \\
\hline FLX-DGN-FS-DG2 & $\begin{array}{l}\text { U.S. NRC } 2015 \text { Parameter Estimation Update } \\
\text { http://nrcoe.inel.gov/resultsdb/ParamEstSpar/ February2017. Section } 3.1\end{array}$ \\
\hline FLX-DGN-LR-DG1 & $\begin{array}{l}\text { U.S. NRC } 2015 \text { Parameter Estimation Update } \\
\text { http://nrcoe.inel.gov/resultsdb/ParamEstSpar/ February2017. Section } 3.1\end{array}$ \\
\hline FLX-DGN-LR-DG2 & $\begin{array}{l}\text { U.S. NRC } 2015 \text { Parameter Estimation Update } \\
\text { http://nrcoe.inel.gov/resultsdb/ParamEstSpar/ February2017. Section } 3.1\end{array}$ \\
\hline FLX-EDP-FR-MUP1 & $\begin{array}{l}\text { U.S. NRC } 2015 \text { Parameter Estimation Update } \\
\text { http://nrcoe.inel.gov/resultsdb/ParamEstSpar/ February2017. Section } 2.3\end{array}$ \\
\hline FLX-EDP-FR-MUP2 & $\begin{array}{l}\text { U.S. NRC } 2015 \text { Parameter Estimation Update } \\
\text { http://nrcoe.inel.gov/resultsdb/ParamEstSpar/ February2017. Section } 2.3\end{array}$ \\
\hline FLX-EDP-FR-SGP1 & $\begin{array}{l}\text { U.S. NRC } 2015 \text { Parameter Estimation Update } \\
\text { http://nrcoe.inel.gov/resultsdb/ParamEstSpar/ February2017. Section } 2.3\end{array}$ \\
\hline FLX-EDP-FR-SGP2 & $\begin{array}{l}\text { U.S. NRC } 2015 \text { Parameter Estimation Update } \\
\text { http://nrcoe.inel.gov/resultsdb/ParamEstSpar/ February2017. Section } 2.3\end{array}$ \\
\hline FLX-EDP-FS-MUP1 & $\begin{array}{l}\text { U.S. NRC } 2015 \text { Parameter Estimation Update } \\
\text { http://nrcoe.inel.gov/resultsdb/ParamEstSpar/ February2017. Section } 2.3\end{array}$ \\
\hline FLX-EDP-FS-MUP2 & $\begin{array}{l}\text { U.S. NRC } 2015 \text { Parameter Estimation Update } \\
\text { http://nrcoe.inel.gov/resultsdb/ParamEstSpar/ February2017. Section } 2.3\end{array}$ \\
\hline FLX-EDP-FS-SGP1 & $\begin{array}{l}\text { U.S. NRC } 2015 \text { Parameter Estimation Update } \\
\text { http://nrcoe.inel.gov/resultsdb/ParamEstSpar/ February2017. Section } 2.3\end{array}$ \\
\hline FLX-EDP-FS-SGP2 & $\begin{array}{l}\text { U.S. NRC } 2015 \text { Parameter Estimation Update } \\
\text { http://nrcoe.inel.gov/resultsdb/ParamEstSpar/ February2017. Section } 2.3\end{array}$ \\
\hline FLX-EDP-LR-MUP1 & $\begin{array}{l}\text { U.S. NRC } 2015 \text { Parameter Estimation Update } \\
\text { http://nrcoe.inel.gov/resultsdb/ParamEstSpar/ February2017. Section } 2.3\end{array}$ \\
\hline FLX-EDP-LR-MUP2 & $\begin{array}{l}\text { U.S. NRC } 2015 \text { Parameter Estimation Update } \\
\text { http://nrcoe.inel.gov/resultsdb/ParamEstSpar/ February2017. Section } 2.3\end{array}$ \\
\hline FLX-EDP-LR-SGP1 & $\begin{array}{l}\text { U.S. NRC } 2015 \text { Parameter Estimation Update } \\
\text { http://nrcoe.inel.gov/resultsdb/ParamEstSpar/ February2017. Section } 2.3\end{array}$ \\
\hline FLX-EDP-LR-SGP2 & $\begin{array}{l}\text { U.S. NRC } 2015 \text { Parameter Estimation Update } \\
\text { http://nrcoe.inel.gov/resultsdb/ParamEstSpar/ February2017. Section } 2.3\end{array}$ \\
\hline HPI-CKV-CC-001 & $\begin{array}{l}\text { U.S. NRC } 2015 \text { Parameter Estimation Update } \\
\text { http://nrcoe.inel.gov/resultsdb/ParamEstSpar/ February2017. }\end{array}$ \\
\hline HPI-CKV-CC-002 & $\begin{array}{l}\text { U.S. NRC } 2015 \text { Parameter Estimation Update } \\
\text { http://nrcoe.inel.gov/resultsdb/ParamEstSpar/ February2017. }\end{array}$ \\
\hline HPI-CKV-CC-003 & $\begin{array}{l}\text { U.S. NRC } 2015 \text { Parameter Estimation Update } \\
\text { http://nrcoe.inel.gov/resultsdb/ParamEstSpar/ February2017. }\end{array}$ \\
\hline HPI-CKV-CC-004 & $\begin{array}{l}\text { U.S. NRC } 2015 \text { Parameter Estimation Update } \\
\text { http://nrcoe.inel.gov/resultsdb/ParamEstSpar/ February2017. }\end{array}$ \\
\hline HPI-CKV-CC-005A & $\begin{array}{l}\text { U.S. NRC } 2015 \text { Parameter Estimation Update } \\
\text { http://nrcoe.inel.gov/resultsdb/ParamEstSpar/ February2017. }\end{array}$ \\
\hline
\end{tabular}


Generic Pressurized Water Reactor (PWR)

U.S. NRC 2015 Parameter Estimation Update

\begin{tabular}{|c|c|}
\hline HPI-CKV-CC-005B & http://nrcoe.inel.gov/resultsdb/ParamEstSpar/ February2017. \\
\hline HPI-CKV-OO-005A & $\begin{array}{l}\text { U.S. NRC } 2015 \text { Parameter Estimation Update } \\
\text { http://nrcoe.inel.gov/resultsdb/ParamEstSpar/ February } 2017 .\end{array}$ \\
\hline HPI-CKV-OO-005B & $\begin{array}{l}\text { U.S. NRC } 2015 \text { Parameter Estimation Update } \\
\text { http://nrcoe.inel.gov/resultsdb/ParamEstSpar/ February } 2017 .\end{array}$ \\
\hline HPI-MOV-CC-006A & $\begin{array}{l}\text { U.S. NRC } 2015 \text { Parameter Estimation Update } \\
\text { http://nrcoe.inel.gov/resultsdb/ParamEstSpar/ February } 2017 .\end{array}$ \\
\hline HPI-MOV-CC-006B & $\begin{array}{l}\text { U.S. NRC } 2015 \text { Parameter Estimation Update } \\
\text { http://nrcoe.inel.gov/resultsdb/ParamEstSpar/ February } 2017 .\end{array}$ \\
\hline HPI-MOV-OO-006A & $\begin{array}{l}\text { U.S. NRC } 2015 \text { Parameter Estimation Update } \\
\text { http://nrcoe.inel.gov/resultsdb/ParamEstSpar/ February2017. }\end{array}$ \\
\hline HPI-MOV-OO-006B & $\begin{array}{l}\text { U.S. NRC } 2015 \text { Parameter Estimation Update } \\
\text { http://nrcoe.inel.gov/resultsdb/ParamEstSpar/ February } 2017 .\end{array}$ \\
\hline HPI-TNK-FC-RWST & $\begin{array}{l}\text { U.S. NRC } 2015 \text { Parameter Estimation Update } \\
\text { http://nrcoe.inel.gov/resultsdb/ParamEstSpar/ February } 2017 .\end{array}$ \\
\hline HPI-XVM-OC-001 & $\begin{array}{l}\text { U.S. NRC } 2015 \text { Parameter Estimation Update } \\
\text { http://nrcoe.inel.gov/resultsdb/ParamEstSpar/ February2017. }\end{array}$ \\
\hline IAS-SYS-FC-SYSTEM & Estimated IAS system comprised of two compressors; one in operation. \\
\hline IE-RCS-MOV-CO-007A & $\begin{array}{l}\text { U.S. NRC } 2015 \text { Parameter Estimation Update } \\
\text { http://nrcoe.inel.gov/resultsdb/ParamEstSpar/ February2017. }\end{array}$ \\
\hline IE-RCS-MOV-CO-007B & $\begin{array}{l}\text { U.S. NRC } 2015 \text { Parameter Estimation Update } \\
\text { http://nrcoe.inel.gov/resultsdb/ParamEstSpar/February2017. }\end{array}$ \\
\hline
\end{tabular}

http://nrcoe.inel.gov/resultsdb/ParamEstSpar/ February2017.

U.S. NRC 2015 Parameter Estimation Update

IE-RCS-MOV-CO-008A http://nrcoe.inel.gov/resultsdb/ParamEstSpar/ February2017.

U.S. NRC 2015 Parameter Estimation Update

IE-RCS-MOV-CO-008B http://nrcoe.inel.gov/resultsdb/ParamEstSpar/ February2017.

ISL-CKV-CC-LPI001 Inter-System Loss Of Coolant Accident (ISLOCA) Bill Galyean November 2005.

ISL-CKV-CC-LPI002 Inter-System Loss Of Coolant Accident (ISLOCA) Bill Galyean November 2005.

ISL-CKV-CC-LPI003 Inter-System Loss Of Coolant Accident (ISLOCA) Bill Galyean November 2005.

ISL-CKV-CC-LPI004 Inter-System Loss Of Coolant Accident (ISLOCA) Bill Galyean November 2005.

ISL-CKV-CC-RCS001 Inter-System Loss Of Coolant Accident (ISLOCA) Bill Galyean November 2005.

ISL-CKV-CC-RCS002 Inter-System Loss Of Coolant Accident (ISLOCA) Bill Galyean November 2005.

ISL-CKV-CC-RCS003 Inter-System Loss Of Coolant Accident (ISLOCA) Bill Galyean November 2005.

ISL-CKV-CC-RCS004 Inter-System Loss Of Coolant Accident (ISLOCA) Bill Galyean November 2005.

ISL-PSF-RP-RHR W. J. Galyean et al. ISLOCA Research Program Final Report NUREG/CR-5928 July 1993. HEP values for diagnosing an Interfacing Systems LOCA (ISLOCA) are from a white paper

ISL-XHE-XD-DIAG written by Bill Galyean of the INL dated November 2005.

ISL-XHE-XE-NONREC 0.1 placeholder

ISL-XHE-XE-REC Generic Operator Action

U.S. NRC 2015 Parameter Estimation Update

LPI-CKV-CC-001 http://nrcoe.inel.gov/resultsdb/ParamEstSpar/ February2017.

U.S. NRC 2015 Parameter Estimation Update

LPI-CKV-CC-001A http://nrcoe.inel.gov/resultsdb/ParamEstSpar/ February2017.

U.S. NRC 2015 Parameter Estimation Update

LPI-CKV-CC-001B ～http://nrcoe.inel.gov/resultsdb/ParamEstSpar/ February2017.

U.S. NRC 2015 Parameter Estimation Update

LPI-CKV-CC-002 http://nrcoe.inel.gov/resultsdb/ParamEstSpar/ February2017.

LPI-CKV-CC-003 U.S. NRC 2015 Parameter Estimation Update

Model Version: 1.0

Page 75 
http://nrcoe.inel.gov/resultsdb/ParamEstSpar/ February2017.

\begin{tabular}{|c|c|}
\hline LPI-CKV-CC-003A & $\begin{array}{l}\text { U.S. NRC } 2015 \text { Parameter Estimation Update } \\
\text { http://nrcoe.inel.gov/resultsdb/ParamEstSpar/ February2017. }\end{array}$ \\
\hline LPI-CKV-CC-003B & $\begin{array}{l}\text { U.S. NRC } 2015 \text { Parameter Estimation Update } \\
\text { http://nrcoe.inel.gov/resultsdb/ParamEstSpar/ February2017. }\end{array}$ \\
\hline LPI-CKV-CC-004 & $\begin{array}{l}\text { U.S. NRC } 2015 \text { Parameter Estimation Update } \\
\text { http://nrcoe.inel.gov/resultsdb/ParamEstSpar/ February2017. }\end{array}$ \\
\hline LPI-MDP-FR-1A & $\begin{array}{l}\text { U.S. NRC } 2015 \text { Parameter Estimation Update } \\
\text { http://nrcoe.inel.gov/resultsdb/ParamEstSpar/ February2017. }\end{array}$ \\
\hline LPI-MDP-FR-1B & $\begin{array}{l}\text { U.S. NRC } 2015 \text { Parameter Estimation Update } \\
\text { http://nrcoe.inel.gov/resultsdb/ParamEstSpar/ February2017. }\end{array}$ \\
\hline LPI-MDP-FS-1A & $\begin{array}{l}\text { U.S. NRC } 2015 \text { Parameter Estimation Update } \\
\text { http://nrcoe.inel.gov/resultsdb/ParamEstSpar/ February } 2017 .\end{array}$ \\
\hline LPI-MDP-FS-1B & $\begin{array}{l}\text { U.S. NRC } 2015 \text { Parameter Estimation Update } \\
\text { http://nrcoe.inel.gov/resultsdb/ParamEstSpar/ February2017. }\end{array}$ \\
\hline LPI-MDP-TM-1A & $\begin{array}{l}2015 \text { Update to the Parameter Estimation Component Unavailability Data Sheets Section } 1 \\
\text { Table 1-1 }\end{array}$ \\
\hline LPI-MDP-TM-1B & $\begin{array}{l}2015 \text { Update to the Parameter Estimation Component Unavailability Data Sheets Section } 1 \\
\text { Table 1-1 }\end{array}$ \\
\hline LPI-MOV-CC-004B & $\begin{array}{l}\text { U.S. NRC } 2015 \text { Parameter Estimation Update } \\
\text { http://nrcoe.inel.gov/resultsdb/ParamEstSpar/ February } 2017 .\end{array}$ \\
\hline LPI-MOV-CC-MINFL005A & $\begin{array}{l}\text { U.S. NRC } 2015 \text { Parameter Estimation Update } \\
\text { http://nrcoe.inel.gov/resultsdb/ParamEstSpar/ February } 2017 .\end{array}$ \\
\hline LPI-MOV-CC-MINFLO05B & $\begin{array}{l}\text { U.S. NRC } 2015 \text { Parameter Estimation Update } \\
\text { http://nrcoe.inel.gov/resultsdb/ParamEstSpar/ February2017. }\end{array}$ \\
\hline LPI-MOV-OC-001A & $\begin{array}{l}\text { U.S. NRC } 2015 \text { Parameter Estimation Update } \\
\text { http://nrcoe.inel.gov/resultsdb/ParamEstSpar/ February2017. }\end{array}$ \\
\hline LPI-MOV-OC-001B & $\begin{array}{l}\text { U.S. NRC } 2015 \text { Parameter Estimation Update } \\
\text { http://nrcoe.inel.gov/resultsdb/ParamEstSpar/ February } 2017 .\end{array}$ \\
\hline LPI-MOV-OC-002A & $\begin{array}{l}\text { U.S. NRC } 2015 \text { Parameter Estimation Update } \\
\text { http://nrcoe.inel.gov/resultsdb/ParamEstSpar/ February2017. }\end{array}$ \\
\hline LPI-MOV-OC-002B & $\begin{array}{l}\text { U.S. NRC } 2015 \text { Parameter Estimation Update } \\
\text { http://nrcoe.inel.gov/resultsdb/ParamEstSpar/ February2017. }\end{array}$ \\
\hline LPI-MOV-OC-004A & $\begin{array}{l}\text { U.S. NRC } 2015 \text { Parameter Estimation Update } \\
\text { http://nrcoe.inel.gov/resultsdb/ParamEstSpar/ February2017. }\end{array}$ \\
\hline LPI-MOV-OC-012A & $\begin{array}{l}\text { U.S. NRC } 2015 \text { Parameter Estimation Update } \\
\text { http://nrcoe.inel.gov/resultsdb/ParamEstSpar/ February2017. }\end{array}$ \\
\hline LPI-MOV-OC-012B & $\begin{array}{l}\text { U.S. NRC } 2015 \text { Parameter Estimation Update } \\
\text { http://nrcoe.inel.gov/resultsdb/ParamEstSpar/ February2017. }\end{array}$ \\
\hline LPI-MOV-OO-001A & $\begin{array}{l}\text { U.S. NRC } 2015 \text { Parameter Estimation Update } \\
\text { http://nrcoe.inel.gov/resultsdb/ParamEstSpar/ February } 2017 .\end{array}$ \\
\hline LPI-MOV-OO-001B & $\begin{array}{l}\text { U.S. NRC } 2015 \text { Parameter Estimation Update } \\
\text { http://nrcoe.inel.gov/resultsdb/ParamEstSpar/ February2017. }\end{array}$ \\
\hline LPI-XVM-OC-007B & $\begin{array}{l}\text { U.S. NRC } 2015 \text { Parameter Estimation Update } \\
\text { http://nrcoe.inel.gov/resultsdb/ParamEstSpar/ February2017. }\end{array}$ \\
\hline LPR-CKV-CC-SICVCA & $\begin{array}{l}\text { U.S. NRC } 2015 \text { Parameter Estimation Update } \\
\text { http://nrcoe.inel.gov/resultsdb/ParamEstSpar/ February2017. }\end{array}$ \\
\hline LPR-CKV-CC-SICVCB & $\begin{array}{l}\text { U.S. NRC } 2015 \text { Parameter Estimation Update } \\
\text { http://nrcoe.inel.gov/resultsdb/ParamEstSpar/ February2017. }\end{array}$ \\
\hline LPR-HTX-LK-A & U.S. NRC 2015 Parameter Estimation Update \\
\hline Model Version: 1.0 & Page 76 \\
\hline
\end{tabular}


http://nrcoe.inel.gov/resultsdb/ParamEstSpar/ February2017.

U.S. NRC 2015 Parameter Estimation Update

LPR-HTX-LK-B http://nrcoe.inel.gov/resultsdb/ParamEstSpar/ February2017.

U.S. NRC 2015 Parameter Estimation Update

LPR-HTX-PG-A http://nrcoe.inel.gov/resultsdb/ParamEstSpar/ February2017.

U.S. NRC 2015 Parameter Estimation Update

LPR-HTX-PG-B http://nrcoe.inel.gov/resultsdb/ParamEstSpar/ February2017.

U.S. NRC 2015 Parameter Estimation Update

LPR-HTX-RP-A http://nrcoe.inel.gov/resultsdb/ParamEstSpar/ February2017.

U.S. NRC 2015 Parameter Estimation Update

LPR-HTX-RP-B http://nrcoe.inel.gov/resultsdb/ParamEstSpar/ February2017.

U.S. NRC 2015 Parameter Estimation Update

LPR-MOV-CC-009A http://nrcoe.inel.gov/resultsdb/ParamEstSpar/ February2017.

U.S. NRC 2015 Parameter Estimation Update

LPR-MOV-CC-009B http://nrcoe.inel.gov/resultsdb/ParamEstSpar/ February2017.

U.S. NRC 2015 Parameter Estimation Update

LPR-MOV-CC-SICVCA http://nrcoe.inel.gov/resultsdb/ParamEstSpar/ February2017.

U.S. NRC 2015 Parameter Estimation Update

LPR-MOV-CC-SICVCB http://nrcoe.inel.gov/resultsdb/ParamEstSpar/ February2017.

U.S. NRC 2015 Parameter Estimation Update

LPR-MOV-OO-004A http://nrcoe.inel.gov/resultsdb/ParamEstSpar/ February2017.

U.S. NRC 2015 Parameter Estimation Update

LPR-MOV-OO-004B http://nrcoe.inel.gov/resultsdb/ParamEstSpar/ February2017.

U.S. NRC 2015 Parameter Estimation Update

MFW-CKV-CC-001 http://nrcoe.inel.gov/resultsdb/ParamEstSpar/ February2017.

U.S. NRC 2015 Parameter Estimation Update

MFW-CKV-CC-002 http://nrcoe.inel.gov/resultsdb/ParamEstSpar/ February2017.

U.S. NRC 2015 Parameter Estimation Update

MFW-CKV-CC-003 http://nrcoe.inel.gov/resultsdb/ParamEstSpar/ February2017.

U.S. NRC 2015 Parameter Estimation Update

MFW-CKV-CC-004 http://nrcoe.inel.gov/resultsdb/ParamEstSpar/ February2017.

U.S. NRC 2015 Parameter Estimation Update

MSS-ARV-CC-001A http://nrcoe.inel.gov/resultsdb/ParamEstSpar/ February2017.

U.S. NRC 2015 Parameter Estimation Update

MSS-ARV-CC-001B http://nrcoe.inel.gov/resultsdb/ParamEstSpar/ February2017.

U.S. NRC 2015 Parameter Estimation Update

MSS-ARV-CC-001C http://nrcoe.inel.gov/resultsdb/ParamEstSpar/ February2017.

U.S. NRC 2015 Parameter Estimation Update

MSS-ARV-CC-001D http://nrcoe.inel.gov/resultsdb/ParamEstSpar/ February2017.

U.S. NRC 2015 Parameter Estimation Update

MSS-MSV-OO-MSIVA http://nrcoe.inel.gov/resultsdb/ParamEstSpar/ February2017.

U.S. NRC 2015 Parameter Estimation Update

MSS-MSV-OO-MSIVB http://nrcoe.inel.gov/resultsdb/ParamEstSpar/ February2017.

U.S. NRC 2015 Parameter Estimation Update

MSS-MSV-OO-MSIVC http://nrcoe.inel.gov/resultsdb/ParamEstSpar/ February2017.

U.S. NRC 2015 Parameter Estimation Update

MSS-MSV-OO-MSIVD http://nrcoe.inel.gov/resultsdb/ParamEstSpar/ February2017.

U.S. NRC 2015 Parameter Estimation Update

MSS-SVV-OO-003A http://nrcoe.inel.gov/resultsdb/ParamEstSpar/ February2017.

MSS-SVV-OO-003B U.S. NRC 2015 Parameter Estimation Update

Model Version: 1.0

Page 77 
http://nrcoe.inel.gov/resultsdb/ParamEstSpar/ February2017.

U.S. NRC 2015 Parameter Estimation Update

MSS-SVV-0O-003C http://nrcoe.inel.gov/resultsdb/ParamEstSpar/ February2017.

U.S. NRC 2015 Parameter Estimation Update

MSS-SVV-OO-003D http://nrcoe.inel.gov/resultsdb/ParamEstSpar/ February2017.

Recommended PORV challenge rates following a LOOP Joe Minarick July 121996 TO:

PORV-PRV-CO-SBO Distribution.

U.S. NRC 2015 Parameter Estimation Update

PORV-PRV-CO-TRANS http://nrcoe.inel.gov/resultsdb/ParamEstSpar/ February2017.

U.S. NRC 2015 Parameter Estimation Update

PPR-AOV-CC-001A http://nrcoe.inel.gov/resultsdb/ParamEstSpar/ February2017.

U.S. NRC 2015 Parameter Estimation Update

PPR-AOV-CC-001B http://nrcoe.inel.gov/resultsdb/ParamEstSpar/ February2017.

U.S. NRC 2015 Parameter Estimation Update

PPR-PRV-CC-001 http://nrcoe.inel.gov/resultsdb/ParamEstSpar/ February2017.

U.S. NRC 2015 Parameter Estimation Update

PPR-PRV-CC-002 http://nrcoe.inel.gov/resultsdb/ParamEstSpar/ February2017.

U.S. NRC 2015 Parameter Estimation Update

PPR-PRV-OO-001 http://nrcoe.inel.gov/resultsdb/ParamEstSpar/ February2017.

U.S. NRC 2015 Parameter Estimation Update

PPR-PRV-OO-001AL http://nrcoe.inel.gov/resultsdb/ParamEstSpar/ February2017.

U.S. NRC 2015 Parameter Estimation Update

PPR-PRV-OO-002 http://nrcoe.inel.gov/resultsdb/ParamEstSpar/ February2017.

U.S. NRC 2015 Parameter Estimation Update

PPR-PRV-OO-002AL http://nrcoe.inel.gov/resultsdb/ParamEstSpar/ February2017.

U.S. NRC 2015 Parameter Estimation Update

PPR-SRV-CC-001 http://nrcoe.inel.gov/resultsdb/ParamEstSpar/ February2017.

U.S. NRC 2015 Parameter Estimation Update

PPR-SRV-CC-002 http://nrcoe.inel.gov/resultsdb/ParamEstSpar/ February2017.

U.S. NRC 2015 Parameter Estimation Update

PPR-SRV-CC-003 http://nrcoe.inel.gov/resultsdb/ParamEstSpar/ February2017.

U.S. NRC 2015 Parameter Estimation Update

PPR-SRV-OO-001 http://nrcoe.inel.gov/resultsdb/ParamEstSpar/ February2017.

U.S. NRC 2015 Parameter Estimation Update

PPR-SRV-OO-002 http://nrcoe.inel.gov/resultsdb/ParamEstSpar/ February2017.

U.S. NRC 2015 Parameter Estimation Update

PPR-SRV-OO-003 http://nrcoe.inel.gov/resultsdb/ParamEstSpar/ February2017.

U.S. NRC 2015 Parameter Estimation Update

RCS-CKV-CC-001 http://nrcoe.inel.gov/resultsdb/ParamEstSpar/ February2017.

U.S. NRC 2015 Parameter Estimation Update

RCS-CKV-CC-002 http://nrcoe.inel.gov/resultsdb/ParamEstSpar/ February2017.

U.S. NRC 2015 Parameter Estimation Update

RCS-CKV-CC-003 http://nrcoe.inel.gov/resultsdb/ParamEstSpar/ February2017.

U.S. NRC 2015 Parameter Estimation Update

RCS-CKV-CC-004 http://nrcoe.inel.gov/resultsdb/ParamEstSpar/ February2017.

U.S. NRC 2015 Parameter Estimation Update

RCS-MOV-CC-007A http://nrcoe.inel.gov/resultsdb/ParamEstSpar/ February2017.

U.S. NRC 2015 Parameter Estimation Update

RCS-MOV-CC-008A http://nrcoe.inel.gov/resultsdb/ParamEstSpar/ February2017.

RCS-MOV-CC-008B U.S. NRC 2015 Parameter Estimation Update 
http://nrcoe.inel.gov/resultsdb/ParamEstSpar/ February2017.

RCS-MOV-CO-007A Inter-System Loss Of Coolant Accident (ISLOCA) Bill Galyean November 2005.

RCS-MOV-CO-007B Inter-System Loss Of Coolant Accident (ISLOCA) Bill Galyean November 2005.

RCS-MOV-CO-008A Inter-System Loss Of Coolant Accident (ISLOCA) Bill Galyean November 2005.

RCS-MOV-CO-008B Inter-System Loss Of Coolant Accident (ISLOCA) Bill Galyean November 2005.

RCS-PHIN-MODPOOR WCP-15831

RCS-POWER-HIGH WCP-15831

RHR-EQ3-EQ

RHR-EQ4-EQ

Estimated from the seismic fragility curve.

RHR-EQ5-EQ

Estimated from the seismic fragility curve.

RPS-CCP-TM-CHA

Estimated from the seismic fragility curve.

U.S. NRC 2015 Parameter Estimation Update
SIS-CKV-CC-001
http://nrcoe.inel.gov/resultsdb/ParamEstSpar/ February2017.

SIS-CKV-CC-002 http://nrcoe.inel.gov/resultsdb/ParamEstSpar/ February2017.

U.S. NRC 2015 Parameter Estimation Update

SIS-CKV-CC-003 http://nrcoe.inel.gov/resultsdb/ParamEstSpar/ February2017.

U.S. NRC 2015 Parameter Estimation Update

SIS-CKV-CC-003A http://nrcoe.inel.gov/resultsdb/ParamEstSpar/ February2017.

U.S. NRC 2015 Parameter Estimation Update

SIS-CKV-CC-003B http://nrcoe.inel.gov/resultsdb/ParamEstSpar/ February2017.

U.S. NRC 2015 Parameter Estimation Update

SIS-CKV-CC-004 http://nrcoe.inel.gov/resultsdb/ParamEstSpar/ February2017.

U.S. NRC 2015 Parameter Estimation Update

SIS-CKV-CC-004A http://nrcoe.inel.gov/resultsdb/ParamEstSpar/ February2017.

U.S. NRC 2015 Parameter Estimation Update

SIS-CKV-CC-004B http://nrcoe.inel.gov/resultsdb/ParamEstSpar/ February2017.

U.S. NRC 2015 Parameter Estimation Update

SIS-MDP-FR-01A http://nrcoe.inel.gov/resultsdb/ParamEstSpar/ February2017.

U.S. NRC 2015 Parameter Estimation Update

SIS-MDP-FR-01B http://nrcoe.inel.gov/resultsdb/ParamEstSpar/ February2017.

U.S. NRC 2015 Parameter Estimation Update

SIS-MDP-FS-01A http://nrcoe.inel.gov/resultsdb/ParamEstSpar/ February2017.

U.S. NRC 2015 Parameter Estimation Update

SIS-MDP-FS-01B http://nrcoe.inel.gov/resultsdb/ParamEstSpar/ February2017.

2015 Update to the Parameter Estimation Component Unavailability Data Sheets Section 1

SIS-MDP-TM-01A Table 1-1

2015 Update to the Parameter Estimation Component Unavailability Data Sheets Section 1

SIS-MDP-TM-01B Table 1-1

U.S. NRC 2015 Parameter Estimation Update

SIS-MOV-OC-001A http://nrcoe.inel.gov/resultsdb/ParamEstSpar/ February2017.

U.S. NRC 2015 Parameter Estimation Update

SIS-MOV-OC-001B http://nrcoe.inel.gov/resultsdb/ParamEstSpar/ February2017.

U.S. NRC 2015 Parameter Estimation Update

SIS-MOV-OC-002A http://nrcoe.inel.gov/resultsdb/ParamEstSpar/ February2017.

U.S. NRC 2015 Parameter Estimation Update

SIS-MOV-OC-002B http://nrcoe.inel.gov/resultsdb/ParamEstSpar/ February2017.

U.S. NRC 2015 Parameter Estimation Update

SIS-MOV-OO-MFA01 http://nrcoe.inel.gov/resultsdb/ParamEstSpar/ February2017.

Model Version: 1.0

Page 79 


\begin{tabular}{|c|c|}
\hline SIS-MOV-OO-MFB01 & $\begin{array}{l}\text { U.S. NRC } 2015 \text { Parameter Estimation Update } \\
\text { http://nrcoe.inel.gov/resultsdb/ParamEstSpar/ February } 2017 .\end{array}$ \\
\hline SWS-CKV-CC-001A & $\begin{array}{l}\text { U.S. NRC } 2015 \text { Parameter Estimation Update } \\
\text { http://nrcoe.inel.gov/resultsdb/ParamEstSpar/ February } 2017 .\end{array}$ \\
\hline SWS-CKV-CC-001B & $\begin{array}{l}\text { U.S. NRC } 2015 \text { Parameter Estimation Update } \\
\text { http://nrcoe.inel.gov/resultsdb/ParamEstSpar/ February } 2017 .\end{array}$ \\
\hline SWS-CKV-CC-001C & $\begin{array}{l}\text { U.S. NRC } 2015 \text { Parameter Estimation Update } \\
\text { http://nrcoe.inel.gov/resultsdb/ParamEstSpar/ February } 2017 .\end{array}$ \\
\hline SWS-MDP-FR-1A & $\begin{array}{l}\text { U.S. NRC } 2015 \text { Parameter Estimation Update } \\
\text { http://nrcoe.inel.gov/resultsdb/ParamEstSpar/ February2017. }\end{array}$ \\
\hline SWS-MDP-FR-1B & $\begin{array}{l}\text { U.S. NRC } 2015 \text { Parameter Estimation Update } \\
\text { http://nrcoe.inel.gov/resultsdb/ParamEstSpar/ February2017. }\end{array}$ \\
\hline SWS-MDP-FR-1C & $\begin{array}{l}\text { U.S. NRC } 2015 \text { Parameter Estimation Update } \\
\text { http://nrcoe.inel.gov/resultsdb/ParamEstSpar/ February2017. }\end{array}$ \\
\hline SWS-MDP-FS-1A & $\begin{array}{l}\text { U.S. NRC } 2015 \text { Parameter Estimation Update } \\
\text { http://nrcoe.inel.gov/resultsdb/ParamEstSpar/ February } 2017 .\end{array}$ \\
\hline SWS-MDP-FS-1B & $\begin{array}{l}\text { U.S. NRC } 2015 \text { Parameter Estimation Update } \\
\text { http://nrcoe.inel.gov/resultsdb/ParamEstSpar/ February2017. }\end{array}$ \\
\hline SWS-MDP-FS-1C & $\begin{array}{l}\text { U.S. NRC } 2015 \text { Parameter Estimation Update } \\
\text { http://nrcoe.inel.gov/resultsdb/ParamEstSpar/ February } 2017 .\end{array}$ \\
\hline SWS-MDP-TM-1A & $\begin{array}{l}2015 \text { Update to the Parameter Estimation Component Unavailability Data Sheets Section } 1 \\
\text { Table 1-1 }\end{array}$ \\
\hline SWS-MDP-TM-1B & $\begin{array}{l}2015 \text { Update to the Parameter Estimation Component Unavailability Data Sheets Section } 1 \\
\text { Table 1-1 }\end{array}$ \\
\hline SWS-MDP-TM-1C & $\begin{array}{l}2015 \text { Update to the Parameter Estimation Component Unavailability Data Sheets Section } 1 \\
\text { Table 1-1 }\end{array}$ \\
\hline SWS-MOV-OC-001A & $\begin{array}{l}\text { U.S. NRC } 2015 \text { Parameter Estimation Update } \\
\text { http://nrcoe.inel.gov/resultsdb/ParamEstSpar/ February2017. }\end{array}$ \\
\hline SWS-MOV-OC-001B & $\begin{array}{l}\text { U.S. NRC } 2015 \text { Parameter Estimation Update } \\
\text { http://nrcoe.inel.gov/resultsdb/ParamEstSpar/ February } 2017 .\end{array}$ \\
\hline SWS-STR-PG-1A & $\begin{array}{l}\text { U.S. NRC } 2015 \text { Parameter Estimation Update } \\
\text { http://nrcoe.inel.gov/resultsdb/ParamEstSpar/ February2017. }\end{array}$ \\
\hline SWS-STR-PG-1B & $\begin{array}{l}\text { U.S. NRC } 2015 \text { Parameter Estimation Update } \\
\text { http://nrcoe.inel.gov/resultsdb/ParamEstSpar/ February2017. }\end{array}$ \\
\hline SWS-STR-PG-1C & $\begin{array}{l}\text { U.S. NRC } 2015 \text { Parameter Estimation Update } \\
\text { http://nrcoe.inel.gov/resultsdb/ParamEstSpar/ February2017. }\end{array}$ \\
\hline SWS-XVM-CO-002B & $\begin{array}{l}\text { U.S. NRC } 2015 \text { Parameter Estimation Update } \\
\text { http://nrcoe.inel.gov/resultsdb/ParamEstSpar/ February } 2017 .\end{array}$ \\
\hline SWS-XVM-OO-002A & $\begin{array}{l}\text { U.S. NRC } 2015 \text { Parameter Estimation Update } \\
\text { http://nrcoe.inel.gov/resultsdb/ParamEstSpar/ February } 2017 .\end{array}$ \\
\hline
\end{tabular}


Generic Pressurized Water Reactor (PWR)

\section{Basic Event Probabilities}

\begin{tabular}{|c|c|c|}
\hline Basic Event Name & Description & $\begin{array}{l}\text { Calculated } \\
\text { Probability }\end{array}$ \\
\hline ACC-CKV-CC-001A & ACC CKV-001A FAILS TO OPEN & $9.2 \mathrm{E}-6$ \\
\hline ACC-CKV-CC-001B & ACC CKV-001B FAILS TO OPEN & $9.2 \mathrm{E}-6$ \\
\hline ACC-CKV-CC-001C & ACC CKV-001C FAILS TO OPEN & $9.2 \mathrm{E}-6$ \\
\hline ACC-CKV-CC-001D & ACC CKV-001D FAILS TO OPEN & $9.2 \mathrm{E}-6$ \\
\hline ACC-CKV-CF-CC001 & CCF OF ACC CKV-001 TO OPEN & $1.2 \mathrm{E}-7$ \\
\hline ACC-MOV-OC-002A & ACC MOV-002A FAILS TO REMAIN OPEN & $7.8 \mathrm{E}-7$ \\
\hline ACC-MOV-OC-002B & ACC MOV-002B FAILS TO REMAIN OPEN & $7.8 \mathrm{E}-7$ \\
\hline ACC-MOV-OC-002C & ACC MOV-002C FAILS TO REMAIN OPEN & $7.8 \mathrm{E}-7$ \\
\hline ACC-MOV-OC-002D & ACC MOV-002D FAILS TO REMAIN OPEN & $7.8 \mathrm{E}-7$ \\
\hline ACC-TNK-EQ1-BE & ACC TANK FAILURE DUE TO SESIMIC EVENT BIN 1 & $6.5 \mathrm{E}-6$ \\
\hline ACC-TNK-EQ2-BE & ACC TANK FAILURE DUE TO SESIMIC EVENT BIN 2 & $6.9 \mathrm{E}-3$ \\
\hline ACC-TNK-EQ3-BE & ACC TANK FAILURE DUE TO SESIMIC EVENT BIN 3 & $8.3 \mathrm{E}-2$ \\
\hline ACC-TNK-EQ4-BE & ACC TANK FAILURE DUE TO SESIMIC EVENT BIN 4 & $2.9 \mathrm{E}-1$ \\
\hline ACC-TNK-EQ5-BE & ACC TANK FAILURE DUE TO SESIMIC EVENT BIN 5 & $6.0 \mathrm{E}-1$ \\
\hline ACC-TNK-EQ6-BE & ACC TANK FAILURE DUE TO SESIMIC EVENT BIN 6 & $9.4 \mathrm{E}-1$ \\
\hline ACC-TNK-FC-ACCA & Accumulator-A Fails to Operate & $4.6 \mathrm{E}-6$ \\
\hline ACC-TNK-FC-ACCB & Accumulator-B Fails to Operate & $4.6 \mathrm{E}-6$ \\
\hline ACC-TNK-FC-ACCC & Accumulator-C Fails to Operate & $4.6 \mathrm{E}-6$ \\
\hline ACC-TNK-FC-ACCD & Accumulator-D Fails to Operate & $4.6 \mathrm{E}-6$ \\
\hline ACC-TNK-LK-ACCA & Accumulator-A External Leakage (Small) & $2.9 \mathrm{E}-6$ \\
\hline ACC-TNK-LK-ACCB & Accumulator-B External Leakage (Small) & $2.9 \mathrm{E}-6$ \\
\hline ACC-TNK-LK-ACCC & Accumulator-C External Leakage (Small) & $2.9 \mathrm{E}-6$ \\
\hline ACC-TNK-LK-ACCD & Accumulator-D External Leakage (Small) & $2.9 \mathrm{E}-6$ \\
\hline ACC-TNK-RP-ACCA & Accumulator-A External Leakage (Rupture) & $2.0 \mathrm{E}-7$ \\
\hline ACC-TNK-RP-ACCB & Accumulator-B External Leakage (Rupture) & $2.0 \mathrm{E}-7$ \\
\hline ACC-TNK-RP-ACCC & Accumulator-C External Leakage (Rupture) & $2.0 \mathrm{E}-7$ \\
\hline ACC-TNK-RP-ACCD & Accumulator-D External Leakage (Rupture) & $2.0 \mathrm{E}-7$ \\
\hline ACP-BAC-EQ1-480V & 480V FAILURE DUE TO SESIMIC EVENT BIN 1 & $5.1 \mathrm{E}-5$ \\
\hline ACP-BAC-EQ1-4KV & 4160V FAILURE DUE TO SESIMIC EVENT BIN 1 & $2.5 \mathrm{E}-6$ \\
\hline ACP-BAC-EQ2-480V & 480V FAILURE DUE TO SESIMIC EVENT BIN 2 & $2.3 \mathrm{E}-2$ \\
\hline ACP-BAC-EQ2-4KV & 4160V FAILURE DUE TO SESIMIC EVENT BIN 2 & $3.8 \mathrm{E}-3$ \\
\hline ACP-BAC-EQ3-480V & 480V FAILURE DUE TO SESIMIC EVENT BIN 3 & $1.8 \mathrm{E}-1$ \\
\hline ACP-BAC-EQ3-4KV & 4160V FAILURE DUE TO SESIMIC EVENT BIN 3 & $5.6 \mathrm{E}-2$ \\
\hline ACP-BAC-EQ4-480V & 480V FAILURE DUE TO SESIMIC EVENT BIN 4 & $4.6 \mathrm{E}-1$ \\
\hline ACP-BAC-EQ4-4KV & 4160V FAILURE DUE TO SESIMIC EVENT BIN 4 & $2.2 \mathrm{E}-1$ \\
\hline ACP-BAC-EQ5-480V & 480V FAILURE DUE TO SESIMIC EVENT BIN 5 & $7.7 \mathrm{E}-1$ \\
\hline ACP-BAC-EQ5-4KV & 4160V FAILURE DUE TO SESIMIC EVENT BIN 5 & $5.2 \mathrm{E}-1$ \\
\hline ACP-BAC-EQ6-480V & 480V FAILURE DUE TO SESIMIC EVENT BIN 6 & $9.8 \mathrm{E}-1$ \\
\hline ACP-BAC-EQ6-4KV & 4160V FAILURE DUE TO SESIMIC EVENT BIN 6 & $9.1 \mathrm{E}-1$ \\
\hline ACP-BAC-LP-1A & 4160 V VITAL AC BUS 1A FAILS & $2.3 \mathrm{E}-5$ \\
\hline ACP-BAC-LP-1B & 4160 V VITAL AC BUS 1B FAILS & $2.3 \mathrm{E}-5$ \\
\hline ACP-BAC-LP-480V1A & 480 V VITAL AC BUS FAILS & $2.3 \mathrm{E}-5$ \\
\hline ACP-BAC-LP-480V1B & $480 \mathrm{~V}$ VITAL AC BUS FAILS & $2.3 \mathrm{E}-5$ \\
\hline ACP-BAC-LP-SWGC & 4160 VAC BUS SWG-C FAILS & $2.3 \mathrm{E}-5$ \\
\hline ACP-BAC-LP-SWY101 & SWITCHYARD-101 AC BUS FAILS & $2.3 \mathrm{E}-5$ \\
\hline ACP-BAC-LP-X1A & 4160 V NON-VITAL AC BUS FAILS & $2.3 \mathrm{E}-5$ \\
\hline ACP-BAC-LP-XB & 4160 V NON-VITAL AC BUS FAILS & $2.3 \mathrm{E}-5$ \\
\hline ACP-CRB-CC-A001 & CRB-A001 FAILS TO STRIP OFF FROM BUS TO PREVENT OVERLOADING BY DG & $2.5 \mathrm{E}-3$ \\
\hline ACP-CRB-CC-A101 & CIRCUIT BREAKER-A101 FAILS TO OPEN FOR TRAIN-A & $2.5 \mathrm{E}-3$ \\
\hline
\end{tabular}




\section{Generic Pressurized Water Reactor (PWR)}

\begin{tabular}{|c|c|c|}
\hline Basic Event Name & Description & $\begin{array}{l}\text { Calculated } \\
\text { Probability }\end{array}$ \\
\hline ACP-CRB-CC-B001 & CRB-B001 FAILS TO STRIP OF FROM BUS TO PREVENT OVERLOADING BY DG & $2.5 \mathrm{E}-3$ \\
\hline ACP-CRB-CC-B101 & CIRCUIT BREAKER-B101 FAILS TO OPEN FOR TRAIN-B & $2.5 \mathrm{E}-3$ \\
\hline ACP-CRB-CO-A103 & CIRCUIT BREAKER-A103 FAILS TO REMAIN CLOSE FOR TRAIN-A & $2.8 \mathrm{E}-6$ \\
\hline ACP-CRB-CO-A105 & CIRCUIT BREAKER-A105 FAILS TO REMAIN CLOSE FOR TRAIN-A & $2.4 \mathrm{E}-6$ \\
\hline ACP-CRB-CO-A106 & CIRCUIT BREAKER 480VAC A106 FAILS TO REMAIN CLOSE FOR TRAIN-A & $2.4 \mathrm{E}-6$ \\
\hline ACP-CRB-CO-B105 & CIRCUIT BREAKER-B105 FAILS TO REMAIN CLOSE FOR TRAIN-B & $2.4 \mathrm{E}-6$ \\
\hline ACP-CRB-CO-B106 & CIRCUIT BREAKER-B106 FAILS TO REMAIN CLOSE FOR TRAIN-B & $2.4 \mathrm{E}-6$ \\
\hline ACP-CRB-OC-B103 & CIRCUIT BREAKER-B103 FAILS TO REMAIN OPEN FOR TRAIN-B & $2.8 \mathrm{E}-6$ \\
\hline ACP-CRB-OO-A102 & CIRCUIT BREAKER-A102 FAILS TO CLOSE FOR TRAIN-A & $2.5 \mathrm{E}-3$ \\
\hline ACP-CRB-OO-B102 & CIRCUIT BREAKER-B102 FAILS TO CLOSE FOR TRAIN-B & $2.5 \mathrm{E}-3$ \\
\hline ACP-CRB-OO-SWGC & CIRCUIT BREAKER SWG-C FAILS TO CLOSE ON DEMAND & $2.0 \mathrm{E}-3$ \\
\hline ACP-TFM-FC-480V1A & 4160/480 VAC TRANSFORMER 1A FAILS TO OPERATE & $6.9 \mathrm{E}-5$ \\
\hline ACP-TFM-FC-480V1B & 4160/480 VAC TRANSFORMER 1B FAILS TO OPERATE & $6.9 \mathrm{E}-5$ \\
\hline ACP-TFM-FC-X1A & TRANSFORMER-101A FAILS TO OPERATE & $6.9 \mathrm{E}-5$ \\
\hline ACP-TFM-FC-XB & TRANSFORMER-101B FAILS TO OPERATE & $6.9 \mathrm{E}-5$ \\
\hline AFW-AOV-OC-TDPSGA & AFW TDP AOV-A TO SG-A FAILS TO REMAIN OPEN & $2.5 \mathrm{E}-6$ \\
\hline AFW-AOV-OC-TDPSGB & AFW TDP AOV TO SG-B FAILS TO REMAIN OPEN & $2.5 \mathrm{E}-6$ \\
\hline AFW-AOV-OC-TDPSGC & AFW TDP AOV TO SG-C FAILS TO REMAIN OPEN & $2.5 \mathrm{E}-6$ \\
\hline AFW-AOV-OC-TDPSGD & AFW TDP AOV SG-D FAILS TO REMAIN OPEN & $2.5 \mathrm{E}-6$ \\
\hline AFW-CKV-CC-001A & AFW INLET CKV-001A TO SG-A FAILS TO OPEN & $9.2 \mathrm{E}-6$ \\
\hline AFW-CKV-CC-001B & AFW INLET CKV-001B TO SG-B FAILS TO OPEN & $9.2 \mathrm{E}-6$ \\
\hline AFW-CKV-CC-001C & AFW INLET CKV-001C TO SG-C FAILS TO OPEN & $9.2 \mathrm{E}-6$ \\
\hline AFW-CKV-CC-001D & AFW INLET CKV-001D TO SG-D FAILS TO OPEN & $9.2 \mathrm{E}-6$ \\
\hline AFW-CKV-CC-002A & AFW MDP-B DISCHARGE CKV-002A TO SG-A FAILS TO OPEN & $9.2 \mathrm{E}-6$ \\
\hline AFW-CKV-CC-002B & AFW MDP-A CKV-002B TO SG-B FAILS TO OPEN & $9.2 \mathrm{E}-6$ \\
\hline AFW-CKV-CC-002C & AFW MDP-A CKV-002C TO SG-C FAILS TO OPEN & $9.2 \mathrm{E}-6$ \\
\hline AFW-CKV-CC-002D & AFW MDP-B CKV-002D TO SG-D FAILS TO OPEN & $9.2 \mathrm{E}-6$ \\
\hline AFW-CKV-CC-003A & AFW TDP CKV-003A TO SG-A FAILS TO OPEN & $9.2 \mathrm{E}-6$ \\
\hline AFW-CKV-CC-003B & AFW TDP CKV-003B SG-B FAILS TO OPEN & $9.2 \mathrm{E}-6$ \\
\hline AFW-CKV-CC-003C & AFW TDP CKV-003C TO SG-C FAILS TO OPEN & $9.2 \mathrm{E}-6$ \\
\hline AFW-CKV-CC-003D & AFW TDP CKV-003D TO SG-D FAILS TO OPEN & $9.2 \mathrm{E}-6$ \\
\hline AFW-CKV-CC-MPA01 & AFW SUCTION CKV-MPA01 FAILS TO OPEN & $9.2 \mathrm{E}-6$ \\
\hline AFW-CKV-CC-MPA02 & AFW DISCHARGE CKV-MPA02 FAILS TO OPEN & $9.2 \mathrm{E}-6$ \\
\hline AFW-CKV-CC-MPB01 & AFW SUCTION CKV-MPB01 FAILS TO OPEN & $9.2 \mathrm{E}-6$ \\
\hline AFW-CKV-CC-MPB02 & AFW DISCHARGE CKV-MPB02 FAILS TO OPEN & $9.2 \mathrm{E}-6$ \\
\hline AFW-CKV-CC-TP01 & AFW SUCTION CKV-TP01 FAILS TO OPEN & $9.2 \mathrm{E}-6$ \\
\hline AFW-CKV-CC-TP02 & AFW DISCHARGE CKV-TP02 FAILS TO OPEN & $9.2 \mathrm{E}-6$ \\
\hline AFW-CKV-CF-001 & CCF OF AFW CKV-001 TO OPEN & $1.2 \mathrm{E}-7$ \\
\hline AFW-CKV-CF-MPTP1 & CCF OF AFW SUCTION CKVS MPA-01 MPB-01 AND TP01 TO OPEN & $6.1 \mathrm{E}-8$ \\
\hline AFW-CKV-CF-MPTP2 & CCF OF AFW DISCHARGE CKV MPA-02 MPB-02 AND TP02 TO OPEN & $6.1 \mathrm{E}-8$ \\
\hline AFW-FCV-OC-MDPA & AFW MDP-A FLOW CONTROL MOV FAILS TO REMAIN OPEN & $7.8 \mathrm{E}-7$ \\
\hline AFW-FCV-OC-MDPB & AFW MDP-B FLOW CONTROL MOV FAILS TO REMAIN OPEN & $7.8 \mathrm{E}-7$ \\
\hline AFW-FCV-OC-TDP & AFW TDP FLOW CONTROL MOV FAILS TO REMAIN OPEN & $7.8 \mathrm{E}-7$ \\
\hline AFW-MDP-CF-FR & CCF OF AFW MDP TO RUN & $1.1 \mathrm{E}-5$ \\
\hline AFW-MDP-CF-FS & CCF OF AFW MDP TO START & $3.1 \mathrm{E}-5$ \\
\hline AFW-MDP-EQ1-BE & AFW MDP FAILURE DUE TO SESIMIC EVENT BIN 1 & $6.5 \mathrm{E}-6$ \\
\hline AFW-MDP-EQ2-BE & AFW MDP FAILURE DUE TO SESIMIC EVENT BIN 2 & $6.9 \mathrm{E}-3$ \\
\hline AFW-MDP-EQ3-BE & AFW MDP FAILURE DUE TO SESIMIC EVENT BIN 3 & $8.3 \mathrm{E}-2$ \\
\hline AFW-MDP-EQ4-BE & AFW MDP FAILURE DUE TO SESIMIC EVENT BIN 4 & $2.9 \mathrm{E}-1$ \\
\hline AFW-MDP-EQ5-BE & AFW MDP FAILURE DUE TO SESIMIC EVENT BIN 5 & $6.0 \mathrm{E}-1$ \\
\hline AFW-MDP-EQ6-BE & AFW MDP FAILURE DUE TO SESIMIC EVENT BIN 6 & $9.4 \mathrm{E}-1$ \\
\hline AFW-MDP-FR-A & AFW MOTOR-DRIVEN PUMP-A FAILS TO RUN & $3.9 \mathrm{E}-4$ \\
\hline
\end{tabular}




\section{Generic Pressurized Water Reactor (PWR)}

\begin{tabular}{|c|c|c|}
\hline Basic Event Name & Description & $\begin{array}{l}\text { Calculated } \\
\text { Probability }\end{array}$ \\
\hline AFW-MDP-FR-B & AFW MOTOR-DRIVEN PUMP-B FAILS TO RUN & $3.9 \mathrm{E}-4$ \\
\hline AFW-MDP-FS-A & AFW MOTOR-DRIVEN PUMP-A FAILS TO START & $7.9 \mathrm{E}-4$ \\
\hline AFW-MDP-FS-B & AFW MOTOR-DRIVEN PUMP-B FAILS TO START & $7.9 \mathrm{E}-4$ \\
\hline AFW-MDP-TM-A & AFW MDP-A UNAVAILABLE DUE TO TEST AND MAINTENANCE & $4.0 \mathrm{E}-3$ \\
\hline AFW-MDP-TM-B & AFW MDP-B UNAVAILABLE DUE TO TEST AND MAINTENANCE & $4.0 \mathrm{E}-3$ \\
\hline AFW-MOV-OC-004A & AFW MDP-B DISCHARGE MOV-004A TO SG-A FAILS TO REMAIN OPEN & $7.8 \mathrm{E}-7$ \\
\hline AFW-MOV-OC-004B & AFW MDP-A MOV-004B TO SG-B FAILS TO REMAIN OPEN & $7.8 \mathrm{E}-7$ \\
\hline AFW-MOV-OC-004C & AFW MDP-A MOV-004C TO SG-C FAILS TO REMAIN OPEN & $7.8 \mathrm{E}-7$ \\
\hline AFW-MOV-OC-004D & AFW MDP-B MOV-004D TO SG-D FAILS TO REMAIN OPEN & $7.8 \mathrm{E}-7$ \\
\hline AFW-PMP-CF-FR & CCF OF AFW PUMPS TO RUN (EXCLUDING DRIVER) & $1.6 \mathrm{E}-5$ \\
\hline AFW-TDP-EQ1-BE & AFW TDP FAILURE DUE TO SESIMIC EVENT BIN 1 & $4.9 \mathrm{E}-4$ \\
\hline AFW-TDP-EQ2-BE & AFW TDP FAILURE DUE TO SESIMIC EVENT BIN 2 & $8.1 \mathrm{E}-2$ \\
\hline AFW-TDP-EQ3-BE & AFW TDP FAILURE DUE TO SESIMIC EVENT BIN 3 & $3.8 \mathrm{E}-1$ \\
\hline AFW-TDP-EQ4-BE & AFW TDP FAILURE DUE TO SESIMIC EVENT BIN 4 & $6.9 \mathrm{E}-1$ \\
\hline AFW-TDP-EQ5-BE & AFW TDP FAILURE DUE TO SESIMIC EVENT BIN 5 & $9.1 \mathrm{E}-1$ \\
\hline AFW-TDP-EQ6-BE & AFW TDP FAILURE DUE TO SESIMIC EVENT BIN 6 & $1.0 \mathrm{E}+0$ \\
\hline AFW-TDP-FR-TDP & AFW TDP FAILS TO RUN & 4.7E-2 \\
\hline AFW-TDP-FS-TDP & AFW TDP FAILS TO START & $6.0 \mathrm{E}-3$ \\
\hline AFW-TDP-TM-TDP & AFW TDP IS IN TEST OR MAINTENANCE & $5.4 \mathrm{E}-3$ \\
\hline AFW-TNK-EQ1-CST & AFW TANK FAILURE DUE TO SESIMIC EVENT BIN 1 & $1.5 \mathrm{E}-4$ \\
\hline AFW-TNK-EQ2-CST & AFW TANK FAILURE DUE TO SESIMIC EVENT BIN 2 & $4.3 \mathrm{E}-2$ \\
\hline AFW-TNK-EQ3-CST & AFW TANK FAILURE DUE TO SESIMIC EVENT BIN 3 & $2.6 \mathrm{E}-1$ \\
\hline AFW-TNK-EQ4-CST & AFW TANK FAILURE DUE TO SESIMIC EVENT BIN 4 & $5.7 \mathrm{E}-1$ \\
\hline AFW-TNK-EQ5-CST & AFW TANK FAILURE DUE TO SESIMIC EVENT BIN 5 & $8.4 \mathrm{E}-1$ \\
\hline AFW-TNK-EQ6-CST & AFW TANK FAILURE DUE TO SESIMIC EVENT BIN 6 & $9.9 \mathrm{E}-1$ \\
\hline AFW-TNK-FC-CST & AFW CST FAILS & $6.3 \mathrm{E}-6$ \\
\hline AFW-TNK-HCN1-CST & AFW CST FAILURE DUE TO HURRICANE BIN 1 & $3.0 \mathrm{E}-8$ \\
\hline AFW-TNK-HCN2-CST & AFW CST FAILURE DUE TO HURRICANE BIN 2 & $5.0 \mathrm{E}-7$ \\
\hline AFW-TNK-HCN3-CST & AFW CST FAILURE DUE TO HURRICANE BIN 3 & $7.0 \mathrm{E}-6$ \\
\hline AFW-TNK-HCN4-CST & AFW CST FAILURE DUE TO HURRICANE BIN 4 & $9.0 \mathrm{E}-5$ \\
\hline AFW-TNK-HWD-CST & AFW CST FAILURE DUE TO HIGH WIND & $2.0 \mathrm{E}-10$ \\
\hline AFW-TNK-TOR1-CST & AFW CST FAILURE DUE TO TORNADO BIN 1 & $2.0 \mathrm{E}-5$ \\
\hline AFW-TNK-TOR2-CST & AFW CST FAILURE DUE TO TORNADO BIN 2 & $4.0 \mathrm{E}-4$ \\
\hline AFW-TNK-TOR3-CST & AFW CST FAILURE DUE TO TORNADO BIN 3 & $6.0 \mathrm{E}-3$ \\
\hline AFW-XHE-XM-CST & OPERATOR FAILS TO REFILL CST & $1.0 \mathrm{E}-4$ \\
\hline AFW-XHE-XM-RECP & OPERATOR FAILS TO START/CONTROL AFW FROM RECP & $1.0 \mathrm{E}-2$ \\
\hline AFW-XHE-XM-TDPMAN & FAILURE TO MANUALLY CONTROL AFW-TDP IN 4-24 HOURS & $3.0 \mathrm{E}-1$ \\
\hline CCW-AOV-OC-HTXA & CCW INLET AOV HTX-A FAILS TO REMAIN OPEN & $2.5 \mathrm{E}-6$ \\
\hline CCW-AOV-OC-HTXB & CCW INLET AOV HTX-B FAILS TO REMAIN OPEN & $2.5 \mathrm{E}-6$ \\
\hline CCW-AOV-OC-HTXDISA & CCW DISCHARGE AOV HTX-A FAILS TO REMAIN OPEN & $2.5 \mathrm{E}-6$ \\
\hline CCW-AOV-OC-HTXDISB & CCW DISCHARGE AOV HTX-B FAILS TO REMAIN OPEN & $2.5 \mathrm{E}-6$ \\
\hline CCW-CKV-CC-002B & CCW-CKV-CC-002B FAILS TO OPEN & $9.2 \mathrm{E}-6$ \\
\hline CCW-HTX-CF-PG & CCF OF HEAT EXCHANGER PLUGGING & $3.5 \mathrm{E}-7$ \\
\hline CCW-HTX-EQ1-BE & CCW HTX FAILURE DUE TO SESIMIC EVENT BIN 1 & $4.9 \mathrm{E}-4$ \\
\hline CCW-HTX-EQ2-BE & CCW HTX FAILURE DUE TO SESIMIC EVENT BIN 2 & $8.1 \mathrm{E}-2$ \\
\hline CCW-HTX-EQ3-BE & CCW HTX FAILURE DUE TO SESIMIC EVENT BIN 3 & $3.8 \mathrm{E}-1$ \\
\hline CCW-HTX-EQ4-BE & CCW HTX FAILURE DUE TO SESIMIC EVENT BIN 4 & $6.9 \mathrm{E}-1$ \\
\hline CCW-HTX-EQ5-BE & CCW HTX FAILURE DUE TO SESIMIC EVENT BIN 5 & $9.1 \mathrm{E}-1$ \\
\hline CCW-HTX-EQ6-BE & CCW HTX FAILURE DUE TO SESIMIC EVENT BIN 6 & $1.0 \mathrm{E}+0$ \\
\hline CCW-HTX-LK-1A & CCW-A Heat Exchanger External Leakage (Small) & $6.7 \mathrm{E}-6$ \\
\hline CCW-HTX-LK-1B & CCW-B Heat Exchanger External Leakage (Small) & $6.7 \mathrm{E}-6$ \\
\hline CCW-HTX-PG-1A & CCW HEAT EXCHANGER 1A PLUGGING & $1.3 \mathrm{E}-5$ \\
\hline
\end{tabular}




\section{Generic Pressurized Water Reactor (PWR)}

\begin{tabular}{|c|c|c|}
\hline Basic Event Name & Description & $\begin{array}{l}\text { Calculated } \\
\text { Probability }\end{array}$ \\
\hline CCW-HTX-PG-1B & CCW HEAT EXCHANGER 1B PLUGGING & $1.3 \mathrm{E}-5$ \\
\hline CCW-HTX-RP-1A & CCW-A Heat Exchanger External Leakage (Rupture) & $1.0 \mathrm{E}-6$ \\
\hline CCW-HTX-RP-1B & CCW-B Heat Exchanger External Leakage (Rupture) & $1.0 \mathrm{E}-6$ \\
\hline CCW-MDP-CF-FTR & CCF OF MDP OF CCW TO RUN & $2.1 \mathrm{E}-7$ \\
\hline CCW-MDP-CF-FTS & CCF OF MDP OF CCW TO START & $1.9 \mathrm{E}-6$ \\
\hline CCW-MDP-EQ1-BE & CCW MDP FAILURE DUE TO SESIMIC EVENT BIN 1 & $6.5 \mathrm{E}-6$ \\
\hline CCW-MDP-EQ2-BE & CCW MDP FAILURE DUE TO SESIMIC EVENT BIN 2 & $6.9 \mathrm{E}-3$ \\
\hline CCW-MDP-EQ3-BE & CCW MDP FAILURE DUE TO SESIMIC EVENT BIN 3 & $8.3 \mathrm{E}-2$ \\
\hline CCW-MDP-EQ4-BE & CCW MDP FAILURE DUE TO SESIMIC EVENT BIN 4 & $2.9 \mathrm{E}-1$ \\
\hline CCW-MDP-EQ5-BE & CCW MDP FAILURE DUE TO SESIMIC EVENT BIN 5 & $6.0 \mathrm{E}-1$ \\
\hline CCW-MDP-EQ6-BE & CCW MDP FAILURE DUE TO SESIMIC EVENT BIN 6 & $9.4 \mathrm{E}-1$ \\
\hline CCW-MDP-FR-1A & CCW MOTOR DRIVEN PUMP 1A FAILS TO RUN & $6.6 \mathrm{E}-5$ \\
\hline CCW-MDP-FR-1B & CCW MOTOR DRIVEN PUMP 1B FAILS TO RUN & $6.6 \mathrm{E}-5$ \\
\hline CCW-MDP-FR-1C & CCW MOTOR DRIVEN PUMP 1C FAILS TO RUN & $6.6 \mathrm{E}-5$ \\
\hline CCW-MDP-FS-1A & CCW MOTOR DRIVEN PUMP 1A FAILS TO START & $8.8 \mathrm{E}-4$ \\
\hline CCW-MDP-FS-1B & CCW MOTOR DRIVEN PUMP 1B FAILS TO START & $8.8 \mathrm{E}-4$ \\
\hline CCW-MDP-FS-1C & CCW MOTOR DRIVEN PUMP 1C FAILS TO START & $8.8 \mathrm{E}-4$ \\
\hline CCW-MDP-TM-1A & CCW MDP-1A IN TEST AND MAINTENANCE & $5.9 \mathrm{E}-3$ \\
\hline CCW-MDP-TM-1B & CCW MDP-1B IN TEST AND MAINTENANCE & $5.9 \mathrm{E}-3$ \\
\hline CCW-MDP-TM-1C & CCW MDP-1C IN TEST AND MAINTENANCE & $5.9 \mathrm{E}-3$ \\
\hline CCW-MOV-OC-001A & CCW MDP-A SUCTION MOV 001A FAILS TO REMAIN OPEN & $7.8 \mathrm{E}-7$ \\
\hline CCW-MOV-OC-001B & CCW MDP-B SUCTION MOV 001B FAILS TO REMAIN OPEN & $7.8 \mathrm{E}-7$ \\
\hline CCW-MOV-OC-001C_A & CCW MDP-C SUCTION MOV 001C (TRAIN A) FAILS TO OPEN & $4.2 \mathrm{E}-4$ \\
\hline CCW-MOV-OC-001C_B & CCW MDP-C SUCTION MOV 001C (TRAIN B) FAILS TO OPEN & $4.2 \mathrm{E}-4$ \\
\hline CCW-MOV-OC-002A & CCW MDP-A DISCHARGE MOV-002A FAILS TO REMAIN OPEN & $7.8 \mathrm{E}-7$ \\
\hline CCW-MOV-OC-002B & CCW MDP-B DISCHARGE MOV-002B FAILS TO REMAIN OPEN & $7.8 \mathrm{E}-7$ \\
\hline CCW-MOV-OC-002C_A & CCW MDP-A DISCHARGE MOV-002C (TRAIN A) FAILS TO OPEN & $4.2 \mathrm{E}-4$ \\
\hline CCW-MOV-OC-002C_B & CCW MDP-A DISCHARGE MOV-002C (TRAIN B) FAILS TO OPEN & $4.2 \mathrm{E}-4$ \\
\hline CCW-TNK-FC-SURGE & CCW SURGE FAILS & $6.3 \mathrm{E}-6$ \\
\hline CCW-TRN-OP-STDYA & CCW PUMP-A TRAIN IS STANDBY TO ESF A FOR POST PRCSING RULE & $5.0 \mathrm{E}-1$ \\
\hline CCW-TRN-OP-STDYB & CCW PUMP-C TRAIN IS STANDBY TO ESF B FOR POST PRCSING RULE & $5.0 \mathrm{E}-1$ \\
\hline CCW-XHE-XM-TRNC & OPERATOR FAILS TO START AND ALIGN CCW TRAIN C & $1.0 \mathrm{E}-3$ \\
\hline CSS-SMP-CF-PG & CCF OF CONTAINMENT SUMP PLUGGING & $2.9 \mathrm{E}-7$ \\
\hline CSS-SMP-PG-A & CONTAINMENT RECIRC SUMP PLUGGING IN TRAIN A & $8.2 \mathrm{E}-6$ \\
\hline CSS-SMP-PG-B & CONTAINMENT RECIRC SUMP PLUGGING IN TRAIN B & $8.2 \mathrm{E}-6$ \\
\hline CVC-CKV-CC-004A & CVC MDP DISCHARGE CKV-004A FAILS TO OPEN & $9.2 \mathrm{E}-6$ \\
\hline CVC-CKV-CC-004B & CVC MDP CKV-004B FAILS TO OPEN & $9.2 \mathrm{E}-6$ \\
\hline CVC-MDP-CF-FR & CCF OF CVC MDP TO RUN & $9.5 \mathrm{E}-6$ \\
\hline CVC-MDP-CF-FS & CCF OF CVC MDP TO START & $6.5 \mathrm{E}-6$ \\
\hline CVC-MDP-EQ1-BE & CVC MDP FAILURE DUE TO SESIMIC EVENT BIN 1 & $6.5 \mathrm{E}-6$ \\
\hline CVC-MDP-EQ2-BE & CVC MDP FAILURE DUE TO SESIMIC EVENT BIN 2 & $6.9 \mathrm{E}-3$ \\
\hline CVC-MDP-EQ3-BE & CVC MDP FAILURE DUE TO SESIMIC EVENT BIN 3 & $8.3 \mathrm{E}-2$ \\
\hline CVC-MDP-EQ4-BE & CVC MDP FAILURE DUE TO SESIMIC EVENT BIN 4 & $2.9 \mathrm{E}-1$ \\
\hline CVC-MDP-EQ5-BE & CVC MDP FAILURE DUE TO SESIMIC EVENT BIN 5 & $6.0 \mathrm{E}-1$ \\
\hline CVC-MDP-EQ6-BE & CVC MDP FAILURE DUE TO SESIMIC EVENT BIN 6 & $9.4 \mathrm{E}-1$ \\
\hline CVC-MDP-FR-001A & CVC CCP-1A IN HPI FAILS TO RUN & $3.9 \mathrm{E}-4$ \\
\hline CVC-MDP-FR-001B & CVC CCP-1B FAILS TO RUN & $3.9 \mathrm{E}-4$ \\
\hline CVC-MDP-FS-001A & CVC CCP-1A IN HPI FAILS TO START & $7.9 \mathrm{E}-4$ \\
\hline CVC-MDP-FS-001B & CVC CCP-1B FAILS TO START & $7.9 \mathrm{E}-4$ \\
\hline CVC-MDP-TM-001A & CVC CCP-001A UNAVAILABLE DUE TO TEST AND MAINTENANCE & $4.2 \mathrm{E}-3$ \\
\hline CVC-MDP-TM-001B & CVC CCP-001B UNAVAILABLE DUE TO TEST AND MAINTENANCE & $4.2 \mathrm{E}-3$ \\
\hline CVC-MOV-CC-TRNA & CVC MOV-008 DISCHARGE FAILS TO OPEN & $4.2 \mathrm{E}-4$ \\
\hline
\end{tabular}




\section{Generic Pressurized Water Reactor (PWR)}

\begin{tabular}{|c|c|c|}
\hline Basic Event Name & Description & $\begin{array}{l}\text { Calculated } \\
\text { Probability }\end{array}$ \\
\hline CVC-MOV-CC-TRNB & CVC MOV-009 DISCHARGE FAILS TO OPEN & $4.2 \mathrm{E}-4$ \\
\hline CVC-MOV-CF-DIS & CCF OF CVC DISCHARGE MOVS & $2.2 \mathrm{E}-6$ \\
\hline CVC-MOV-OC-001 & CVC MOV-001 FAILS TO REMAIN OPEN & $7.8 \mathrm{E}-7$ \\
\hline CVC-MOV-OC-002 & CVC MOV-002 FAILS TO REMAIN OPEN & $7.8 \mathrm{E}-7$ \\
\hline CVC-XHE-XM-BORATION & OPERATOR FAILS TO INITIATE EMERGENCY BORATION & $2.0 \mathrm{E}-2$ \\
\hline $\begin{array}{l}\text { CVC-XHE-XM-BORATION- } \\
\text { EQ3 }\end{array}$ & OPERATOR FAILS TO INITIATE EMERGENCY BORATION (EQ-BIN-3) & $5.0 \mathrm{E}-3$ \\
\hline $\begin{array}{l}\text { CVC-XHE-XM-BORATION-H- } \\
\text { LD }\end{array}$ & OPERATOR FAILS TO INITIATE EMERGENCY BORATION (DEPENDENCY) & $1.4 \mathrm{E}-1$ \\
\hline DCP-BAT-CF-ALL & CCF OF ALL BATTERY TOP OPERATE & $1.6 \mathrm{E}-7$ \\
\hline DCP-BAT-EQ1-BE & DCP BATTERY FAILURE DUE TO SESIMIC EVENT BIN 1 & $4.9 \mathrm{E}-4$ \\
\hline DCP-BAT-EQ2-BE & DCP BATTERY FAILURE DUE TO SESIMIC EVENT BIN 2 & $8.1 \mathrm{E}-2$ \\
\hline DCP-BAT-EQ3-BE & DCP BATTERY FAILURE DUE TO SESIMIC EVENT BIN 3 & $3.8 \mathrm{E}-1$ \\
\hline DCP-BAT-EQ4-BE & DCP BATTERY FAILURE DUE TO SESIMIC EVENT BIN 4 & $6.9 \mathrm{E}-1$ \\
\hline DCP-BAT-EQ5-BE & DCP BATTERY FAILURE DUE TO SESIMIC EVENT BIN 5 & $9.1 \mathrm{E}-1$ \\
\hline DCP-BAT-EQ6-BE & DCP BATTERY FAILURE DUE TO SESIMIC EVENT BIN 6 & $1.0 \mathrm{E}+0$ \\
\hline DCP-BAT-LP-A01 & BATTERY A01 FAILS TO OPERATE & $8.9 \mathrm{E}-6$ \\
\hline DCP-BAT-LP-B01 & BATTERY B01 FAILS TO OPERATE & $8.9 \mathrm{E}-6$ \\
\hline DCP-BAT-LP-SCBAT & SWITCHYARD BATTERY FAILS & $8.0 \mathrm{E}-6$ \\
\hline DCP-BCH-CF-ALL & CCF OF ALL BATTERY CHARGER TO OPERATE & 7.9E-7 \\
\hline DCP-BCH-EQ1-BE & DCP BATTERY CHARGER FAILURE DUE TO SESIMIC EVENT BIN 1 & $7.9 \mathrm{E}-8$ \\
\hline DCP-BCH-EQ2-BE & DCP BATTERY CHARGER FAILURE DUE TO SESIMIC EVENT BIN 2 & $4.1 \mathrm{E}-4$ \\
\hline DCP-BCH-EQ3-BE & DCP BATTERY CHARGER FAILURE DUE TO SESIMIC EVENT BIN 3 & $1.2 \mathrm{E}-2$ \\
\hline DCP-BCH-EQ4-BE & DCP BATTERY CHARGER FAILURE DUE TO SESIMIC EVENT BIN 4 & $7.4 \mathrm{E}-2$ \\
\hline DCP-BCH-EQ5-BE & DCP BATTERY CHARGER FAILURE DUE TO SESIMIC EVENT BIN 5 & $2.6 \mathrm{E}-1$ \\
\hline DCP-BCH-EQ6-BE & DCP BATTERY CHARGER FAILURE DUE TO SESIMIC EVENT BIN 6 & $7.5 \mathrm{E}-1$ \\
\hline DCP-BCH-FC-A01 & BATTERY CHARGER A01 FAILS TO OPERATE & $6.2 \mathrm{E}-5$ \\
\hline DCP-BCH-FC-B01 & BATTERY CHARGER B01 FAILS TO OPERATE & $6.2 \mathrm{E}-5$ \\
\hline DCP-BCH-TM-A01 & BATTERY CHARGER A01 IS IN TEST OR MAINTENANCE & $2.0 \mathrm{E}-3$ \\
\hline DCP-BCH-TM-B01 & BATTERY CHARGER B01 IS IN TEST OR MAINTENANCE & $2.0 \mathrm{E}-3$ \\
\hline DCP-BDC-LP-1A & 125V VITAL DC BUS 1A FAILS & $5.2 \mathrm{E}-6$ \\
\hline DCP-BDC-LP-1B & 125V VITAL DC BUS 1B FAILS & $5.2 \mathrm{E}-6$ \\
\hline DCP-BDC-LP-SWGC & 125 VDC PANEL SWG-C FAILS & $5.2 \mathrm{E}-6$ \\
\hline DGR-08H-FT & DIESEL GENERATOR RECOVERY SHORT TERM & $1.0 \mathrm{E}+0$ \\
\hline EPS-CRB-OO-A104 & CIRCUIT BREAKER-A104 FAILS TO CLOSE FOR TRAIN-A & $2.5 \mathrm{E}-3$ \\
\hline EPS-CRB-OO-B104 & CIRCUIT BREAKER-B104 FAILS TO CLOSE FOR TRAIN-B & $2.5 \mathrm{E}-3$ \\
\hline EPS-DGN-CF-FR & CCF OF DIESEL GENERATORS TO RUN & $5.4 \mathrm{E}-4$ \\
\hline EPS-DGN-CF-FS & CCF OF DIESEL GENERATORS TO START & $2.8 \mathrm{E}-5$ \\
\hline EPS-DGN-CF-FTLR & CCF OF DIESEL GENERATORS TO LOAD AND RUN EARLY TERM & $2.1 \mathrm{E}-5$ \\
\hline EPS-DGN-EQ1-BE & EPS DGN FAILURE DUE TO SESIMIC EVENT BIN 1 & $6.5 \mathrm{E}-6$ \\
\hline EPS-DGN-EQ2-BE & EPS DGN FAILURE DUE TO SESIMIC EVENT BIN 2 & $6.9 \mathrm{E}-3$ \\
\hline EPS-DGN-EQ3-BE & EPS DGN FAILURE DUE TO SESIMIC EVENT BIN 3 & 8.3E-2 \\
\hline EPS-DGN-EQ4-BE & EPS DGN FAILURE DUE TO SESIMIC EVENT BIN 4 & $2.9 \mathrm{E}-1$ \\
\hline EPS-DGN-EQ5-BE & EPS DGN FAILURE DUE TO SESIMIC EVENT BIN 5 & $6.0 \mathrm{E}-1$ \\
\hline EPS-DGN-EQ6-BE & EPS DGN FAILURE DUE TO SESIMIC EVENT BIN 6 & $9.4 \mathrm{E}-1$ \\
\hline EPS-DGN-FR-DGNA & DGN-A FAILS TO RUN & $3.4 \mathrm{E}-2$ \\
\hline EPS-DGN-FR-DGNB & DGN-B FAILS TO RUN & $3.4 \mathrm{E}-2$ \\
\hline EPS-DGN-FR-SBO & SBO DG FAILS TO RUN & $3.4 \mathrm{E}-2$ \\
\hline EPS-DGN-FS-DGNA & DGN-A FAILS TO START & $2.9 \mathrm{E}-3$ \\
\hline EPS-DGN-FS-DGNB & DGN-B FAILS TO START & $2.9 \mathrm{E}-3$ \\
\hline EPS-DGN-FS-SBO & SBO DG FAILS TO START & $2.9 \mathrm{E}-3$ \\
\hline EPS-DGN-LR-DGNA & DGN-A FAILS TO LOAD AND RUN, EARLY TERM & 3.7E-3 \\
\hline EPS-DGN-LR-DGNB & DGN-B FAILS TO LOAD AND RUN, EARLY TERM & $3.7 \mathrm{E}-3$ \\
\hline
\end{tabular}




\section{Generic Pressurized Water Reactor (PWR)}

\begin{tabular}{|c|c|c|}
\hline Basic Event Name & Description & $\begin{array}{l}\text { Calculated } \\
\text { Probability }\end{array}$ \\
\hline EPS-DGN-LR-SBO & SBO DG FAILS TO LOAD/RUN & $3.7 \mathrm{E}-3$ \\
\hline EPS-DGN-TM-DGNA & EMERGENCY DIESEL GENERATOR DGN-A TEST OR MAINTENANCE & $1.3 \mathrm{E}-2$ \\
\hline EPS-DGN-TM-DGNB & EMERGENCY DIESEL GENERATOR DGN-B TEST OR MAINTENANCE & $1.3 \mathrm{E}-2$ \\
\hline EPS-DGN-TM-SBO & SBO DIESEL GENERATOR TEST OR MAINTENANCE & $1.3 \mathrm{E}-2$ \\
\hline EPS-FAN-FR-SBOHVAC & SBO DIESEL GENERATOR HVAC UNIT FAILS TO RUN & 4.6E-3 \\
\hline EPS-FAN-FS-SBOHVAC & SBO DIESEL GENERATOR HVAC UNIT FAILS TO START & $6.5 \mathrm{E}-4$ \\
\hline EPS-FAN-LR-SBOHVAC & SBO DIESEL GENERATOR HVAC UNIT FAILS TO LOAD/RUN & $3.8 \mathrm{E}-4$ \\
\hline EPS-SEQ-CF-DGAB & CCF of DG1A \& DG1B Sequencers To Operate & $3.5 \mathrm{E}-6$ \\
\hline EPS-SEQ-FC-DGNA & FAILURE OF SEQUENCER OF DGN-A & $1.1 \mathrm{E}-4$ \\
\hline EPS-SEQ-FC-DGNB & FAILURE OF SEQUENCER OF DGN-B & $1.1 \mathrm{E}-4$ \\
\hline EPS-TNK-EQ1-DGN & EPS DAY TANK FAILURE DUE TO SESIMIC EVENT BIN 1 & $4.1 \mathrm{E}-7$ \\
\hline EPS-TNK-EQ2-DGN & EPS DAY TANK FAILURE DUE TO SESIMIC EVENT BIN 2 & $1.2 \mathrm{E}-3$ \\
\hline EPS-TNK-EQ3-DGN & EPS DAY TANK FAILURE DUE TO SESIMIC EVENT BIN 3 & $2.6 \mathrm{E}-2$ \\
\hline EPS-TNK-EQ4-DGN & EPS DAY TANK FAILURE DUE TO SESIMIC EVENT BIN 4 & $1.3 \mathrm{E}-1$ \\
\hline EPS-TNK-EQ5-DGN & EPS DAY TANK FAILURE DUE TO SESIMIC EVENT BIN 5 & $3.8 \mathrm{E}-1$ \\
\hline EPS-TNK-EQ6-DGN & EPS DAY TANK FAILURE DUE TO SESIMIC EVENT BIN 6 & $8.4 \mathrm{E}-1$ \\
\hline EPS-XHE-XL-NR01H & FAILURE TO RECOVER AN EMERGENCY DIESEL IN 1 HOUR & $8.9 \mathrm{E}-1$ \\
\hline EPS-XHE-XL-NR02H & FAILURE TO RECOVER AN EMERGENCY DIESEL IN 2 HOURS & $8.2 \mathrm{E}-1$ \\
\hline EPS-XHE-XL-NR08H & FAILURE TO RECOVER AN EMERGENCY DIESEL IN 8 HOURS & $6.0 \mathrm{E}-1$ \\
\hline EPS-XHE-XL-NR24H & OPERATOR FAILS TO RECOVER EMERGENCY DIESEL IN 24 HOURS & $3.3 \mathrm{E}-1$ \\
\hline EPS-XHE-XL-NR24H8 & Operator Fails to Recover Emergency Diesel in 24 Hours (Given failure at 8) & $5.5 \mathrm{E}-1$ \\
\hline EPS-XHE-XL-NR72H & OPERATOR FAILS TO RECOVER EMERGENCY DIESEL IN 72 HOURS & $9.2 \mathrm{E}-2$ \\
\hline EPS-XHE-XL-NR72H8 & $\begin{array}{l}\text { OPERATOR FAILS TO RECOVER EMERGENCY DIESEL IN } 72 \text { HOURS (GIVEN } \\
\text { FAILURE AT 8) }\end{array}$ & $1.5 \mathrm{E}-1$ \\
\hline EPS-XHE-XM-DGSEQ & Operator Fails To Recover Sequencer & $1.0 \mathrm{E}+0$ \\
\hline EPS-XHE-XM-RECP & OPERATOR FAILS TO START EDG FROM RECP & $1.0 \mathrm{E}-2$ \\
\hline EPS-XHE-XM-SBODG & OPERATOR FAILS TO START AND ALIGN SBO DIESEL GENERATOR & $4.0 \mathrm{E}-2$ \\
\hline FLI-XHE-XM-25MIN & OPERATOR FAILS TO ISOLATE FLOOD WITHIN 25 MINUTES & $1.0 \mathrm{E}-1$ \\
\hline FLX-BLDG-EQ1-BE & FLEX Building Failure in Seismic BIN-1 & $5.1 \mathrm{E}-5$ \\
\hline FLX-BLDG-EQ2-BE & FLEX Building Failure in Seismic BIN-2 & $2.3 \mathrm{E}-2$ \\
\hline FLX-BLDG-EQ3-BE & FLEX Building Failure in Seismic BIN-3 & $1.8 \mathrm{E}-1$ \\
\hline FLX-BLDG-EQ4-BE & FLEX Building Failure in Seismic BIN-4 & $4.6 \mathrm{E}-1$ \\
\hline FLX-BLDG-EQ5-BE & FLEX Building Failure in Seismic BIN-5 & $7.7 \mathrm{E}-1$ \\
\hline FLX-BLDG-EQ6-BE & FLEX Building Failure in Seismic BIN-6 & $9.8 \mathrm{E}-1$ \\
\hline FLX-DGN-CF-FR & CCF OF FLEX DIESEL GENERATORS TO RUN & $5.4 \mathrm{E}-4$ \\
\hline FLX-DGN-CF-FS & CCF OF FLEX DIESEL GENERATORS TO START & $2.7 \mathrm{E}-5$ \\
\hline FLX-DGN-CF-LR & CCF OF FLEX DGS TO LOAD AND RUN EARLY TERM & $2.1 \mathrm{E}-5$ \\
\hline FLX-DGN-FR-DG1 & FLEX DG 1 FAILS TO RUN & $3.4 \mathrm{E}-2$ \\
\hline FLX-DGN-FR-DG2 & FLEX DG 2 FAILS TO RUN & $3.4 \mathrm{E}-2$ \\
\hline FLX-DGN-FS-DG1 & FLEX DG 1 FAILS TO START & $2.9 \mathrm{E}-3$ \\
\hline FLX-DGN-FS-DG2 & FLEX DG 2 FAILS TO START & $2.9 \mathrm{E}-3$ \\
\hline FLX-DGN-LR-DG1 & FLEX DG 1 FAILS TO LOAD AND RUN, EARLY TERM & $3.7 \mathrm{E}-3$ \\
\hline FLX-DGN-LR-DG2 & FLEX DG 2 FAILS TO LOAD AND RUN, EARLY TERM & 3.7E-3 \\
\hline FLX-EDP-CF-FRMUP & CCF OF FLEX RCS MAKEUP PUMPS TO RUN & $1.2 \mathrm{E}-3$ \\
\hline FLX-EDP-CF-FRSGP & CCF OF FLEX SG PUMPS TO RUN & $1.2 \mathrm{E}-3$ \\
\hline FLX-EDP-CF-FSMUP & CCF OF FLEX RCS MAKEUP SG PUMPS TO START & $6.3 \mathrm{E}-5$ \\
\hline FLX-EDP-CF-FSSGP & CCF OF FLEX SG PUMPS TO START & $6.3 \mathrm{E}-5$ \\
\hline FLX-EDP-CF-LRMUP & CCF OF FLEX RCS MAKEUP PUMPS TO LOAD AND RUN, EARLY & $2.8 \mathrm{E}-5$ \\
\hline FLX-EDP-CF-LRSGP & CCF OF FLEX SG PUMPS TO LOAD AND RUN, EARLY & $2.8 \mathrm{E}-5$ \\
\hline FLX-EDP-FR-MUP1 & FLEX RCS MAKEUP PUMP 1 FAILS TO RUN & $4.1 \mathrm{E}-2$ \\
\hline FLX-EDP-FR-MUP2 & FLEX RCS MAKEUP PUMP 2 FAILS TO RUN & $4.1 \mathrm{E}-2$ \\
\hline FLX-EDP-FR-SGP1 & FLEX SG PUMP 1 FAILS TO RUN & $4.1 \mathrm{E}-2$ \\
\hline
\end{tabular}




\section{Generic Pressurized Water Reactor (PWR)}

\begin{tabular}{|c|c|c|}
\hline Basic Event Name & Description & $\begin{array}{l}\text { Calculated } \\
\text { Probability }\end{array}$ \\
\hline FLX-EDP-FR-SGP2 & FLEX SG PUMP 2 FAILS TO RUN & $4.1 \mathrm{E}-2$ \\
\hline FLX-EDP-FS-MUP1 & FLEX RCS MAKEUP PUMP 1 FAILS TO START & $2.2 \mathrm{E}-3$ \\
\hline FLX-EDP-FS-MUP2 & FLEX RCS MAKEUP PUMP 2 FAILS TO START & $2.2 \mathrm{E}-3$ \\
\hline FLX-EDP-FS-SGP1 & FLEX SG PUMP 1 FAILS TO START & $2.2 \mathrm{E}-3$ \\
\hline FLX-EDP-FS-SGP2 & FLEX SG PUMP 2 FAILS TO START & $2.2 \mathrm{E}-3$ \\
\hline FLX-EDP-LR-MUP1 & FLEX RCS MAKEUP PUMP 1 FAILS TO LOAD AND RUN, EARLY TERM & $9.8 \mathrm{E}-4$ \\
\hline FLX-EDP-LR-MUP2 & FLEX RCS MAKEUP PUMP 2 FAILS TO LOAD AND RUN, EARLY TERM & $9.8 \mathrm{E}-4$ \\
\hline FLX-EDP-LR-SGP1 & FLEX SG PUMP 1 FAILS TO LOAD AND RUN, EARLY TERM & $9.8 \mathrm{E}-4$ \\
\hline FLX-EDP-LR-SGP2 & FLEX SG PUMP 2 FAILS TO LOAD AND RUN, EARLY TERM & $9.8 \mathrm{E}-4$ \\
\hline FLX-SGP-REFILL & Failure of alternate water supply to FLEX-SG pump & $0.0 \mathrm{E}+0$ \\
\hline FLX-XHE-XE-ELAP & Operators fail to declare ELAP when beneficial & $1.0 \mathrm{E}-2$ \\
\hline FLX-XHE-XM-480 & Operators fail to stage or run or load or refuel $480 \mathrm{~V}$ portable FLEX diesel & 1.0E-2 \\
\hline FLX-XHE-XM-MUP & Operators fail to inject boron and RCS makeup by FLEX pump (stage, run, supply, refuel) & $1.0 \mathrm{E}-2$ \\
\hline FLX-XHE-XM-SGP & Operators fail to stage or run or supply or refill FLEX SG pump & $1.0 \mathrm{E}-2$ \\
\hline FRI-PRV-SO-001AB & SPURIOUSLY OPENING OF PORV 001A/B GIVEN A FIRE & $1.0 \mathrm{E}-1$ \\
\hline HCN-SWY-LP-SWYRD-1 & SWITCHYARD FAILURE DUE TO HURRICANE WIND BIN 1 & $2.0 \mathrm{E}-4$ \\
\hline HCN-SWY-LP-SWYRD-2 & SWITCHYARD FAILURE DUE TO HURRICANE WIND BIN 2 & $4.0 \mathrm{E}-3$ \\
\hline HCN-SWY-LP-SWYRD-3 & SWITCHYARD FAILURE DUE TO HURRICANE WIND BIN 3 & $8.0 \mathrm{E}-2$ \\
\hline HCN-SWY-LP-SWYRD-4 & SWITCHYARD FAILURE DUE TO HURRICANE WIND BIN 4 & $1.6 \mathrm{E}-1$ \\
\hline HE-ATWS & HOUSE EVENT - ATWS EVENT HAS OCCURED & $0.0 \mathrm{E}+0$ \\
\hline HE-EQ1 & HOUSE EVENT FOR SEISMIC EVENT BIN 1 & $0.0 \mathrm{E}+0$ \\
\hline HE-EQ2 & HOUSE EVENT FOR SEISMIC EVENT BIN 2 & $0.0 \mathrm{E}+0$ \\
\hline HE-EQ3 & HOUSE EVENT FOR SEISMIC EVENT BIN 3 & $0.0 \mathrm{E}+0$ \\
\hline HE-EQ4 & HOUSE EVENT FOR SEISMIC EVENT BIN 4 & $0.0 \mathrm{E}+0$ \\
\hline HE-EQ5 & HOUSE EVENT FOR SEISMIC EVENT BIN 5 & $0.0 \mathrm{E}+0$ \\
\hline HE-EQ6 & HOUSE EVENT FOR SEISMIC EVENT BIN 6 & $0.0 \mathrm{E}+0$ \\
\hline HE-FLD-4160VACA & HOUSE EVENT - FLOOD IN 4160 V ELECTRICAL ROOM A & $0.0 \mathrm{E}+0$ \\
\hline HE-FLD-4160VACB & HOUSE EVENT - FLOOD IN 4160 V ELECTRICAL ROOM B & $0.0 \mathrm{E}+0$ \\
\hline HE-FLD-AFW & HOUSE EVENT - FLOOD IN AUXILIARY PUMP ROOMS & $0.0 \mathrm{E}+0$ \\
\hline HE-FLD-CCW & HOUSE EVENT - FLOOD IN CCW PUMP ROOMS & $0.0 \mathrm{E}+0$ \\
\hline HE-FLD-CCWA & HOUSE EVENT - FLOOD IN CCW PUMP A ROOM & $0.0 \mathrm{E}+0$ \\
\hline HE-FLD-CCWB & HOUSE EVENT - FLOOD IN CCW PUMP B ROOM & $0.0 \mathrm{E}+0$ \\
\hline HE-FLD-CVC & HOUSE EVENT - FLOOD IN CHARGING ROOM & $0.0 \mathrm{E}+0$ \\
\hline HE-FLD-RHR & HOUSE EVENT - FLOOD IN RHR PUMP ROOMS & $0.0 \mathrm{E}+0$ \\
\hline HE-FLD-SWS & HOUSE EVENT - FLOOD IN SWS PUMP ROOMS & $0.0 \mathrm{E}+0$ \\
\hline HE-FLD-SWSA & HOUSE EVENT - FLOOD IN SWS PUMP A ROOM & $0.0 \mathrm{E}+0$ \\
\hline HE-FLD-SWSB & HOUSE EVENT - FLOOD IN SWS PUMP B ROOM & $0.0 \mathrm{E}+0$ \\
\hline HE-FRI-AB-SIS & HOUSE EVENT - FIRE IN SIS ROOM AND SPURIOUS PORV & $0.0 \mathrm{E}+0$ \\
\hline HE-FRI-SWS-BLD & HOUSE EVENT - FIRE IN SWS BUILDING & $0.0 \mathrm{E}+0$ \\
\hline HE-HCN1 & HOUSE EVENT FOR HURRICANE EVENT BIN 1 & $0.0 \mathrm{E}+0$ \\
\hline HE-HCN2 & HOUSE EVENT FOR HURRICANE EVENT BIN 2 & $0.0 \mathrm{E}+0$ \\
\hline HE-HCN3 & HOUSE EVENT FOR HURRICANE EVENT BIN 3 & $0.0 \mathrm{E}+0$ \\
\hline HE-HCN4 & HOUSE EVENT FOR HURRICANE EVENT BIN 4 & $0.0 \mathrm{E}+0$ \\
\hline HE-HWD & HIGH WIND EVENT OCCURS (HOUSE EVENT) & $0.0 \mathrm{E}+0$ \\
\hline HE-LO4160ACA & House event for loss of $4160 \mathrm{~V}$ BUS A & $0.0 \mathrm{E}+0$ \\
\hline HE-LO4160ACB & House event for loss of $4160 \mathrm{~V}$ BUS B & $0.0 \mathrm{E}+0$ \\
\hline HE-LOCA & LOCA INITIATING EVENT HAS OCCURRED & $0.0 \mathrm{E}+0$ \\
\hline HE-LOCCW & House event for total loss of CCW & $0.0 \mathrm{E}+0$ \\
\hline HE-LODCA & House event for loss of $125 \mathrm{~V}$ DC BUS A & $0.0 \mathrm{E}+0$ \\
\hline HE-LODCB & House event for loss of $125 \mathrm{~V}$ DC BUS B & $0.0 \mathrm{E}+0$ \\
\hline HE-LOMFW & HOUSE EVENT - TOTAL LOSS OF MAIN FEEDWATER INITIATOR & $0.0 \mathrm{E}+0$ \\
\hline HE-LOOP & LOOP EVENT OCCURS (HOUSE EVENT) & $0.0 \mathrm{E}+0$ \\
\hline
\end{tabular}




\section{Generic Pressurized Water Reactor (PWR)}

\begin{tabular}{|c|c|c|}
\hline Basic Event Name & Description & $\begin{array}{l}\text { Calculated } \\
\text { Probability }\end{array}$ \\
\hline HE-LOOP-A & LOOP EVENT FOR TRAIN-A OCCURS (HOUSE EVENT) & $0.0 \mathrm{E}+0$ \\
\hline HE-LOOP-B & LOOP EVENT FOR TRAIN-B OCCURS (HOUSE EVENT) & $0.0 \mathrm{E}+0$ \\
\hline HE-LOOPAV & AVERAGE LOOP EVENT OCCURS (HOUSE EVENT) & $0.0 \mathrm{E}+0$ \\
\hline HE-LOOPGR & GRID RELATED LOOP EVENT OCCURS (HOUSE EVENT) & $0.0 \mathrm{E}+0$ \\
\hline HE-LOOPPC & PLANT CENTERED LOOP EVENT OCCURS (HOUSE EVENT) & $0.0 \mathrm{E}+0$ \\
\hline HE-LOOPSC & SWITCHYARD CENTERED LOOP EVENT OCCURS (HOUSE EVENT) & $0.0 \mathrm{E}+0$ \\
\hline HE-LOOPWR & WEATHER RELATED LOOP EVENT OCCURS (HOUSE EVENT) & $0.0 \mathrm{E}+0$ \\
\hline HE-LOSWS & House event for total loss of SWS & $0.0 \mathrm{E}+0$ \\
\hline HE-LSSB & LARGE STEAM LINE BREAK INITIATING EVENT HAS OCCURRED & $0.0 \mathrm{E}+0$ \\
\hline HE-SBO & LOOP EVENT FOLLOWED BY SBO OCCURS (HOUSE EVENT) & $0.0 \mathrm{E}+0$ \\
\hline HE-SGTR & SGTR INITIATING EVENT HAS OCCURRED & $0.0 \mathrm{E}+0$ \\
\hline HE-SLOCA & SMALL LOCA INITIATING EVENT HAS OCCURRED & $0.0 \mathrm{E}+0$ \\
\hline HE-TOR1 & HOUSE EVENT FOR TORNADO EVENT BIN 1 & $0.0 \mathrm{E}+0$ \\
\hline HE-TOR2 & HOUSE EVENT FOR TORNADO EVENT BIN 2 & $0.0 \mathrm{E}+0$ \\
\hline HE-TOR3 & HOUSE EVENT FOR TORNADO EVENT BIN 3 & $0.0 \mathrm{E}+0$ \\
\hline HPI-CKV-CC-001 & CVC CKV-001 INTO RCS FAILS TO OPEN & $9.2 \mathrm{E}-6$ \\
\hline HPI-CKV-CC-002 & CVC CKV-002 INTO RCS FAILS TO OPEN & $9.2 \mathrm{E}-6$ \\
\hline HPI-CKV-CC-003 & CVC CKV-003 INTO RCS FAILS TO OPEN & $9.2 \mathrm{E}-6$ \\
\hline HPI-CKV-CC-004 & CVC CKV-004 INTO RCS FAILS TO OPEN & $9.2 \mathrm{E}-6$ \\
\hline HPI-CKV-CC-005A & CKV-005A IN HPI FAILS TO OPEN & $9.2 \mathrm{E}-6$ \\
\hline HPI-CKV-CC-005B & CKV-005B IN HPI FAILS TO OPEN & $9.2 \mathrm{E}-6$ \\
\hline HPI-CKV-CF-004 & CCF OF HPI CKV-004 TO FAIL OPEN & $1.9 \mathrm{E}-7$ \\
\hline HPI-CKV-CF-005 & CCF OF HPI CKV-005 TO FAIL OPEN & $1.9 \mathrm{E}-7$ \\
\hline HPI-CKV-CF-CVCINLT & CCF OF CVC INLET CKVS INTO RCS & $1.2 \mathrm{E}-7$ \\
\hline HPI-CKV-OO-005A & CKV-005A IN HPI FAILS TO CLOSE & $1.6 \mathrm{E}-4$ \\
\hline HPI-CKV-OO-005B & CKV-005B IN HPI FAILS TO CLOSE & $1.6 \mathrm{E}-4$ \\
\hline HPI-MOV-CC-006A & MOV-006A IN HPI FAILS TO OPEN & $4.2 \mathrm{E}-4$ \\
\hline HPI-MOV-CC-006B & MOV-006B IN HPI FAILS TO OPEN & $4.2 \mathrm{E}-4$ \\
\hline HPI-MOV-CF-CC-006 & CCF OF HPI MOV-006 TO OPEN & $2.2 \mathrm{E}-6$ \\
\hline HPI-MOV-OO-006A & MOV-006A IN HPI FAILS TO CLOSE & $3.3 \mathrm{E}-4$ \\
\hline HPI-MOV-OO-006B & MOV-006B IN HPI FAILS TO CLOSE & $3.3 \mathrm{E}-4$ \\
\hline HPI-TNK-FC-RWST & RWST TANK FAILS & $6.3 \mathrm{E}-6$ \\
\hline HPI-TNK-HCN1-RWST & RWST FAILURE DUE TO HURRICANE BIN 1 & $3.0 \mathrm{E}-8$ \\
\hline HPI-TNK-HCN2-RWST & RWST FAILURE DUE TO HURRICANE BIN 2 & $5.0 \mathrm{E}-7$ \\
\hline HPI-TNK-HCN3-RWST & RWST FAILURE DUE TO HURRICANE BIN 3 & $7.0 \mathrm{E}-6$ \\
\hline HPI-TNK-HCN4-RWST & RWST FAILURE DUE TO HURRICANE BIN 4 & $9.0 \mathrm{E}-5$ \\
\hline HPI-TNK-HWD-RWST & RWST FAILURE DUE TO HIGH WIND & $2.0 \mathrm{E}-10$ \\
\hline HPI-TNK-TOR1-RWST & RWST FAILURE DUE TO TORNADO BIN 1 & $2.0 \mathrm{E}-5$ \\
\hline HPI-TNK-TOR2-RWST & RWST FAILURE DUE TO TORNADO BIN 2 & $4.0 \mathrm{E}-4$ \\
\hline HPI-TNK-TOR3-RWST & RWST FAILURE DUE TO TORNADO BIN 3 & $6.0 \mathrm{E}-3$ \\
\hline HPI-XHE-XM-FAB & OPERATOR FAILS TO INITIATE FEED AND BLEED COOLING & $2.0 \mathrm{E}-2$ \\
\hline HPI-XHE-XM-FAB-EQ3 & OPERATOR FAILS TO INITIATE FEED AND BLEED COOLING (EQ-BIN-3) & $1.0 \mathrm{E}-1$ \\
\hline HPI-XHE-XM-FAB-H-HD & OPERATOR FAILS TO INITIATE FEED AND BLEED COOLING (DEPENDENCY) & $5.1 \mathrm{E}-1$ \\
\hline HPI-XHE-XM-RWSTR & OPERATORS FAILS TO REFILL RWST & $1.0 \mathrm{E}-3$ \\
\hline HPI-XHE-XM-RWSTR1 & OPERATORS FAILS TO REFILL RWST (DEPENDENCY) & $1.0 \mathrm{E}+0$ \\
\hline HPI-XHE-XM-THRTL & OPERATOR FAILS TO CONTROL/TERMINATE SI & $1.0 \mathrm{E}-3$ \\
\hline HPI-XVM-OC-001 & RWST XVM-001 FAILS TO REMAIN OPEN & $1.2 \mathrm{E}-6$ \\
\hline HPR-XHE-XM-RC & OPERATOR FAILS TO START RECIRC MODE & $1.0 \mathrm{E}-3$ \\
\hline HPR-XHE-XM-RC-H-CD & OPERATOR FAILS TO START RECIRC MODE (DEPENDENT) & $1.0 \mathrm{E}+0$ \\
\hline HWD-SWY-LP-SWYRD & SWITCHYARD FAILURE DUE TO HIGH WIND & $1.0 \mathrm{E}-5$ \\
\hline IAS-SYS-FC-SYSTEM & INSTRUMENT AIR ROLLED UP SYSTEM & $2.5 \mathrm{E}-4$ \\
\hline IE-CCW-HTX-CF-PG & CCF OF CCW HTXs 1A AND 1B TO PLUG (INITIATING EVENT) & $5.4 \mathrm{E}-5$ \\
\hline
\end{tabular}




\section{Generic Pressurized Water Reactor (PWR)}

\begin{tabular}{|c|c|c|}
\hline Basic Event Name & Description & $\begin{array}{l}\text { Calculated } \\
\text { Probability }\end{array}$ \\
\hline IE-CCW-HTX-PG-HX1A & CCW HTX-1A PLUGS (INITIATING EVENT) & $2.0 \mathrm{E}-3$ \\
\hline IE-CCW-HTX-PG-HX1B & CCW HTX-1B PLUGS (INITIATING EVENT) & $2.0 \mathrm{E}-3$ \\
\hline IE-CCW-MDP-CF-FR & CCF OF CCW MDPs 1A, 1B, AND 1C TO RUN (INITIATING EVENT) & $5.2 \mathrm{E}-5$ \\
\hline IE-CCW-MDP-FR-1A & CcW MDP-1A FAILS TO RUN - INITIATING EVENT & $1.0 \mathrm{E}-3$ \\
\hline IE-CCW-MDP-FR-1B & CcW MDP-1B FAILS TO RUN - INITIATING EVENT & $1.0 \mathrm{E}-3$ \\
\hline IE-CCW-MDP-FR-1C & CcW MDP-1C FAILS TO RUN - INITIATING EVENT & $1.0 \mathrm{E}-3$ \\
\hline IE-CCW-TNK-FC-SURGE & FAILURE OF CCW SURGE TANK (INITIATING EVENT) & $5.0 \mathrm{E}-4$ \\
\hline IE-EQ-BIN1 & SEISMIC EVENT IN BIN 1 (0.1 - 0.3g) OCCURS (BIN PGA 0.17) & $1.0 \mathrm{E}-4$ \\
\hline IE-EQ-BIN2 & SEISMIC EVENT IN BIN 2 (0.3 - 0.5g) OCCURS (BIN PGA 0.39) & $8.3 \mathrm{E}-6$ \\
\hline IE-EQ-BIN3 & SEISMIC EVENT IN BIN $3(0.5$ - 0.75g) OCCURS (BIN PGA 0.61) & $2.2 \mathrm{E}-6$ \\
\hline IE-EQ-BIN4 & SEISMIC EVENT IN BIN $4(0.75-1.0 \mathrm{~g})$ OCCURS (BIN PGA 0.87) & $6.1 \mathrm{E}-7$ \\
\hline IE-EQ-BIN5 & SEISMIC EVENT IN BIN 5 (1.0 - 1.5g) OCCURS (BIN PGA 1.22) & $3.3 \mathrm{E}-7$ \\
\hline IE-EQ-BIN6 & SEISMIC EVENT IN BIN 6 (1.5 - 3.0g) OCCURS (BIN PGA 2.12) & $1.2 \mathrm{E}-7$ \\
\hline IE-EQ-BIN7 & SEISMIC EVENT IN BIN 7 (> 3.0g) OCCURS (BIN PGA 3) & $1.0 \mathrm{E}-8$ \\
\hline IE-FLI-4160VACA & PIPING RUPTURE IN 4160V AC ROOM A & $3.0 \mathrm{E}-4$ \\
\hline IE-FLI-4160VACB & PIPING RUPTURE IN 4160V AC ROOM B & $3.0 \mathrm{E}-4$ \\
\hline IE-FLI-AFW-RM & IF - AFW PUMP ROOMS & $2.0 \mathrm{E}-5$ \\
\hline IE-FLI-CCW-RM & IF - CCW PUMP ROOMS (IE) & $2.0 \mathrm{E}-4$ \\
\hline IE-FLI-CCW-RMA & IF - CCW PUMP ROOM A (IE) & $1.0 \mathrm{E}-3$ \\
\hline IE-FLI-CCW-RMB & IF - CCW PUMP ROOM B (IE) & $1.0 \mathrm{E}-3$ \\
\hline IE-FLI-CVC-RM & IF - CVC PUMP ROOM (IE) & $7.0 \mathrm{E}-5$ \\
\hline IE-FLI-RHR-RM & IF - RHR PUMP ROOM (IE) & $8.0 \mathrm{E}-7$ \\
\hline IE-FLI-SWS-RM & IF - SWS PUMP ROOMS (IE) & $5.0 \mathrm{E}-5$ \\
\hline IE-FLI-SWS-RMA & IF - SWS PUMP ROOM A (IE) & $4.0 \mathrm{E}-4$ \\
\hline IE-FLI-SWS-RMB & IF - SWS PUMP ROOM B (IE) & $4.0 \mathrm{E}-4$ \\
\hline IE-FRI-AB-AFWAB & FIRE IN AUX BUILDING CAUSING FAILURE OF AFW MDPS & $2.0 \mathrm{E}-4$ \\
\hline IE-FRI-AB-CCWBC & FIRE IN AUX BUILDING CAUSING FAILURE OF CCW MDP B/C & $8.0 \mathrm{E}-4$ \\
\hline IE-FRI-AB-LOOP & FIRE IN AUX BUILDING CAUSES LOOP & $7.0 \mathrm{E}-6$ \\
\hline IE-FRI-AB-LOOP-DIVA & FIRE IN AUX BUILDING CAUSES LOOP AND LOSS OF DIV A AC & $2.0 \mathrm{E}-3$ \\
\hline IE-FRI-AB-LOOP-DIVB & FIRE IN AUX BUILDING CAUSES LOOP AND LOSS OF DIV B AC & $2.0 \mathrm{E}-3$ \\
\hline IE-FRI-AB-RHRA & FIRE IN AUX BUILDING CAUSING FAILURE OF RHR TRAIN A & $2.0 \mathrm{E}-3$ \\
\hline IE-FRI-AB-SIS & FIRE IN AUX BUILDING CAUSING FAILURE OF SIS TRAINS & $3.0 \mathrm{E}-3$ \\
\hline IE-FRI-AB-SL & FIRE IN AUX BUILDING CAUSING SPURIOUS PORV OPENING & $8.0 \mathrm{E}-5$ \\
\hline IE-FRI-MCR-EVAC & FIRE IN MAIN CONTROL ROOM CAUSES EVACUATION & $3.0 \mathrm{E}-5$ \\
\hline IE-FRI-SWS-BLD & FIRE IN SW BUILDING FAIL SWS PMPS & $6.0 \mathrm{E}-6$ \\
\hline IE-HCN-BIN1 & HURRICANE WIND EVENT (111 - 135 MPH) OCCURS & $2.0 \mathrm{E}-2$ \\
\hline IE-HCN-BIN2 & HURRICANE WIND EVENT (136 - 165 MPH) OCCURS & $3.0 \mathrm{E}-3$ \\
\hline IE-HCN-BIN3 & HURRICANE WIND EVENT (165 - 200 MPH) OCCURS & $8.0 \mathrm{E}-5$ \\
\hline IE-HCN-BIN4 & HURRICANE WIND EVENT (> 200 MPH) OCCURS & $3.0 \mathrm{E}-6$ \\
\hline IE-HWD-96MPH & HIGH WIND EVENT (<96MPH) OCCURS & $1.0 \mathrm{E}-1$ \\
\hline IE-L4160ACA & LOSS OF SAFETY- RELATED BUS 4160 vAC A & $2.9 \mathrm{E}-3$ \\
\hline IE-L4160ACB & LOSS OF SAFETY-RELATED BUS 4160 vAC B & $2.9 \mathrm{E}-3$ \\
\hline IE-LLOCA & LARGE BREAK LOCA & $5.9 \mathrm{E}-6$ \\
\hline IE-LOCCW & TOTAL LOSS OF COMPONENT COOLING WATER & $1.0 \mathrm{E}+0$ \\
\hline IE-LODCA & LOSS OF 125 VDC BUS A & $5.0 \mathrm{E}-4$ \\
\hline IE-LODCB & LOSS OF 125 VDC BUS B & $5.0 \mathrm{E}-4$ \\
\hline IE-LOMFW & TRANSIENTS WITH LOSS OF MFW & $5.9 \mathrm{E}-2$ \\
\hline IE-LOOPAV & LOSS OF OFFSITE POWER (FREQUENCY WEIGHTED AVERAGE) & $3.1 \mathrm{E}-2$ \\
\hline IE-LOOPGR & LOSS OF OFFSITE POWER INITIATOR (GRID-RELATED) & $1.1 \mathrm{E}-2$ \\
\hline IE-LOOPPC & LOSS OF OFFSITE POWER INITIATOR (PLANT- CENTERED) & $2.3 \mathrm{E}-3$ \\
\hline IE-LOOPSC & LOSS OF OFFSITE POWER INITIATOR (SWITCHYARD- CENTERED) & $1.4 \mathrm{E}-2$ \\
\hline IE-LOOPWR & LOSS OF OFFSITE POWER INITIATOR (WEATHER- RELATED) & $6.4 \mathrm{E}-3$ \\
\hline
\end{tabular}




\section{Generic Pressurized Water Reactor (PWR)}

\begin{tabular}{|c|c|c|}
\hline Basic Event Name & Description & $\begin{array}{l}\text { Calculated } \\
\text { Probability }\end{array}$ \\
\hline IE-LSSB & LARGE STEAM LINE BREAK (UNISOLABLE INSIDE CONTAINMENT) & $3.0 \mathrm{E}-4$ \\
\hline IE-MLOCA & MEDIUM BREAK LOCA & $1.5 \mathrm{E}-4$ \\
\hline IE-RCS-MOV-CO-007A & RCS MOV-007A FAILS TO REMAIN CLOSE (ISLOCA INITIATOR) & $2.8 \mathrm{E}-4$ \\
\hline IE-RCS-MOV-CO-007B & RCS MOV-007B FAILS TO REMAIN CLOSE (ISLOCA INITIATOR) & $2.8 \mathrm{E}-4$ \\
\hline IE-RCS-MOV-CO-008A & RCS MOV-008A FAILS TO REMAIN CLOSE (ISLOCA INITIATOR) & $2.8 \mathrm{E}-4$ \\
\hline IE-RCS-MOV-CO-008B & RCS MOV-008B FAILS TO REMAIN CLOSE (ISLOCA INITIATOR) & $2.8 \mathrm{E}-4$ \\
\hline IE-SGTR & STEAM GENERATOR TUBE RUPTURE & $1.7 \mathrm{E}-3$ \\
\hline IE-SLOCA & SMALL BREAK LOCA & $4.0 \mathrm{E}-4$ \\
\hline IE-TORN-BIN1 & TORNADO EVENT (136 - $165 \mathrm{MPH}$ ) OCCURS & $3.0 \mathrm{E}-6$ \\
\hline IE-TORN-BIN2 & TORNADO EVENT (166 - $200 \mathrm{MPH}$ ) OCCURS & $6.0 \mathrm{E}-7$ \\
\hline IE-TORN-BIN3 & TORNADO EVENT (>200 MPH) OCCURS & $4.0 \mathrm{E}-8$ \\
\hline IE-TRANS & TRANSIENTS WITH MFW AVAILABLE & $6.8 \mathrm{E}-1$ \\
\hline IE-XLOCA & EXCESSIVE LOCA & $1.0 \mathrm{E}-7$ \\
\hline IEFT-ISL-RHR-CL-FT & ISLOCA IN RHR DISCHARGE TO CL & $0.0 \mathrm{E}+0$ \\
\hline IEFT-ISL-RHR-HLS-FT & ISLOCA FROM RHR SUCTION FROM HL & $9.0 \mathrm{E}-8$ \\
\hline ISL-CKV-CC-LPI001 & LPI CKV-001 IN CL 1 (ISLOCA) & $2.3 \mathrm{E}-5$ \\
\hline ISL-CKV-CC-LPI002 & LPI CKV-001 IN CL 2 (ISLOCA) & $2.3 \mathrm{E}-5$ \\
\hline ISL-CKV-CC-LPI003 & LPI CKV-001 IN CL 3 (ISLOCA) & $2.3 \mathrm{E}-5$ \\
\hline ISL-CKV-CC-LPI004 & LPI CKV-001 IN CL 4 (ISLOCA) & $2.3 \mathrm{E}-5$ \\
\hline ISL-CKV-CC-RCS001 & RCS CKV-001 IN CL 1 (ISLOCA) & $2.3 \mathrm{E}-5$ \\
\hline ISL-CKV-CC-RCS002 & RCS CKV-001 IN CL 2 (ISLOCA) & $2.3 \mathrm{E}-5$ \\
\hline ISL-CKV-CC-RCS003 & RCS CKV-001 IN CL 3 (ISLOCA) & $2.3 \mathrm{E}-5$ \\
\hline ISL-CKV-CC-RCS004 & RCS CKV-001 IN CL 4 (ISLOCA) & $2.3 \mathrm{E}-5$ \\
\hline ISL-PSF-RP-LPI & LPI INJECTION PIPE RUPTURES & $1.0 \mathrm{E}-1$ \\
\hline ISL-PSF-RP-RHR & RHR INJECTION PIPE RUPTURES & $1.0 \mathrm{E}+0$ \\
\hline ISL-XHE-XD-DIAG & OPERATOR FAILS TO DIAGNOSE ISLOCA & 4.0E-2 \\
\hline ISL-XHE-XE-NONREC & ISLOCA NON-RECOVERY & $1.0 \mathrm{E}-1$ \\
\hline ISL-XHE-XE-NRECRHR & ISLOCA NON-RECOVERY & $1.0 \mathrm{E}-1$ \\
\hline ISL-XHE-XE-REC & OPERATOR FAILS TO ISOLATE(RECOVER) ISLOCA & $4.0 \mathrm{E}-3$ \\
\hline ISL-XHE-XE-RECRHR & OPERATOR FAILS TO ISOLATE(RECOVER) ISLOCA & $1.0 \mathrm{E}-1$ \\
\hline LOOP-EQ1-BE & LOOP OCCURS - SEISMIC BIN 1 & $9.7 \mathrm{E}-2$ \\
\hline LOOP-EQ2-BE & LOOP OCCURS - SEISMIC BIN 2 & $7.3 \mathrm{E}-1$ \\
\hline LOOP-EQ3-BE & LOOP OCCURS - SEISMIC BIN 3 & $9.5 \mathrm{E}-1$ \\
\hline LOOP-EQ4-BE & LOOP OCCURS - SEISMIC BIN 4 & $9.9 \mathrm{E}-1$ \\
\hline LOOP-EQ5-BE & LOOP OCCURS - SEISMIC BIN 5 & $1.0 \mathrm{E}+0$ \\
\hline LOOP-EQ6-BE & LOOP OCCURS - SEISMIC BIN 6 & $1.0 \mathrm{E}+0$ \\
\hline LPI-CKV-CC-001 & LPI RCS CKV-001 FAILS TO OPEN & $9.2 \mathrm{E}-6$ \\
\hline LPI-CKV-CC-001A & LPI MDP-A DISCHARGE CKV-001A FAILS TO OPEN & $9.2 \mathrm{E}-6$ \\
\hline LPI-CKV-CC-001B & LPI MDP-B DISCHARGE CKV-001B FAILS TO OPEN & $9.2 \mathrm{E}-6$ \\
\hline LPI-CKV-CC-002 & LPI RCS CKV-002 FAILS TO OPEN & $9.2 \mathrm{E}-6$ \\
\hline LPI-CKV-CC-003 & LPI RCS CKV-003 FAILS TO OPEN & $9.2 \mathrm{E}-6$ \\
\hline LPI-CKV-CC-003A & LPI MDP-A SUCTION CKV-003A FAILS TO OPEN & $9.2 \mathrm{E}-6$ \\
\hline LPI-CKV-CC-003B & LPI MDP-B SUCTION CKV-003B FAILS TO OPEN & $9.2 \mathrm{E}-6$ \\
\hline LPI-CKV-CC-004 & LPI RCS CKV-004 FAILS TO OPEN & $9.2 \mathrm{E}-6$ \\
\hline LPI-CKV-CF-CC003 & CCF OF LPI CKV-003 TO FAIL OPEN & $1.9 \mathrm{E}-7$ \\
\hline LPI-CKV-CF-RCSCL & CCF OF LPI COLD LEG DISCHARGE CKVS & $1.2 \mathrm{E}-7$ \\
\hline LPI-MDP-CF-FR & CCF OF LPI MDP TO RUN & $2.1 \mathrm{E}-5$ \\
\hline LPI-MDP-CF-FS & CCF OF LPI MDP TO START & $3.0 \mathrm{E}-5$ \\
\hline LPI-MDP-FR-1A & LPI MOTOR-DRIVEN PUMP-1A FAILS TO RUN & $3.9 \mathrm{E}-4$ \\
\hline LPI-MDP-FR-1B & LPI MOTOR-DRIVEN PUMP-1B FAILS TO RUN & $3.9 \mathrm{E}-4$ \\
\hline LPI-MDP-FS-1A & LPI MOTOR-DRIVEN PUMP-1A FAILS TO START & $7.9 \mathrm{E}-4$ \\
\hline LPI-MDP-FS-1B & LPI MOTOR-DRIVEN PUMP-1B FAILS TO START & $7.9 \mathrm{E}-4$ \\
\hline
\end{tabular}




\section{Generic Pressurized Water Reactor (PWR)}

\begin{tabular}{|c|c|c|}
\hline Basic Event Name & Description & $\begin{array}{l}\text { Calculated } \\
\text { Probability }\end{array}$ \\
\hline LPI-MDP-TM-1A & LPI MDP-01A IS IN TEST OR MAINTENANCE & $4.2 \mathrm{E}-3$ \\
\hline LPI-MDP-TM-1B & LPI MDP-01B IS IN TEST OR MAINTENANCE & $4.2 \mathrm{E}-3$ \\
\hline LPI-MOV-CC-004B & LPI MDP-B SUCTION MOV-004B FAILS TO OPEN & $2.5 \mathrm{E}-4$ \\
\hline LPI-MOV-CC-MINFL005A & LPI MDP-A MINFLOW MOV-005A FAILS TO OPEN & $2.5 \mathrm{E}-4$ \\
\hline LPI-MOV-CC-MINFL005B & LPI MDP-B MINFLOW MOV-005B FAILS TO OPEN & $4.2 \mathrm{E}-4$ \\
\hline LPI-MOV-CF-MINFLW & CCF OF LPI MDP MINFLOW MOVS-5A/5B TO OPEN & $4.6 \mathrm{E}-6$ \\
\hline LPI-MOV-OC-001A & LPI MOV-001A FAILS TO REMAIN OPEN & $7.8 \mathrm{E}-7$ \\
\hline LPI-MOV-OC-001B & LPI MOV-001B FAILS TO REMAIN OPEN & $7.8 \mathrm{E}-7$ \\
\hline LPI-MOV-OC-002A & LPI MOV-002A FAILS TO REMAIN OPEN & $7.8 \mathrm{E}-7$ \\
\hline LPI-MOV-OC-002B & LPI MOV-002B FAILS TO REMAIN OPEN & $7.8 \mathrm{E}-7$ \\
\hline LPI-MOV-OC-004A & LPI MDP-A RWST SUCTION MOV-004A FAILS TO REMAIN OPEN & $7.8 \mathrm{E}-7$ \\
\hline LPI-MOV-OC-012A & LPI MDP-A DISCHARGE FLOW CONTROL MOV 012A FAILS TO REMAIN OPEN & $7.8 \mathrm{E}-7$ \\
\hline LPI-MOV-OC-012B & LPI MDP-B DISCHARGE FLOW CONTROL MOV-012B FAILS TO REMAIN OPEN & $7.8 \mathrm{E}-7$ \\
\hline LPI-MOV-OO-001A & LPI MOV-001A FAILS TO CLOSE & $3.3 \mathrm{E}-4$ \\
\hline LPI-MOV-OO-001B & LPI MOV-001B FAILS TO CLOSE & $3.3 \mathrm{E}-4$ \\
\hline LPI-XVM-OC-007B & LPI XVM-007B FAILS TO REMAIN OPEN & $1.2 \mathrm{E}-6$ \\
\hline LPR-CKV-CC-SICVCA & LPR DISCHARGE SI/CVC SUCTION CKV A & $9.2 \mathrm{E}-6$ \\
\hline LPR-CKV-CC-SICVCB & LPR DISCHARGE SI/CVC SUCTION CKV B & $9.2 \mathrm{E}-6$ \\
\hline LPR-HTX-LK-A & LPR-A Heat Exchanger External Leakage (Small) & $6.7 \mathrm{E}-6$ \\
\hline LPR-HTX-LK-B & LPR-B Heat Exchanger External Leakage (Small) & $6.7 \mathrm{E}-6$ \\
\hline LPR-HTX-PG-A & LPR-A Heat Exchanger Plugging/Heat Transfer (Pooled) & $9.2 \mathrm{E}-6$ \\
\hline LPR-HTX-PG-B & LPR-B Heat Exchanger Plugging/Heat Transfer (Pooled) & $9.2 \mathrm{E}-6$ \\
\hline LPR-HTX-RP-A & LPR-A Heat Exchanger External Leakage (Rupture) & $1.0 \mathrm{E}-6$ \\
\hline LPR-HTX-RP-B & LPR-B Heat Exchanger External Leakage (Rupture) & $1.0 \mathrm{E}-6$ \\
\hline LPR-MOV-CC-009A & LPI MDP-A SUMP SUCTION MOV-009A FAILS TO OPEN & $4.2 \mathrm{E}-4$ \\
\hline LPR-MOV-CC-009B & LPI MDP-B SUMP SUCTION MOV-009B FAILS TO OPEN & $4.2 \mathrm{E}-4$ \\
\hline LPR-MOV-CC-SICVCA & LPR DISCHARGE TO SI/CVC SUCTION MOV A & $4.2 \mathrm{E}-4$ \\
\hline LPR-MOV-CC-SICVCB & LPR DISCHARGE TO SI/CVC SUCTION MOV B & $4.2 \mathrm{E}-4$ \\
\hline LPR-MOV-CF-CC-009 & CCF OF LPR MOV-009 TO OPEN & $7.8 \mathrm{E}-6$ \\
\hline LPR-MOV-CF-CVCSUC & CCF OF LPR DISCHARGE SI/CVC SUCTION MOVS & $2.2 \mathrm{E}-6$ \\
\hline LPR-MOV-OO-004A & LPI MDP-A RWST SUCTION MOV-004A FAILS TO CLOSE & $3.3 \mathrm{E}-4$ \\
\hline LPR-MOV-OO-004B & LPI MDP-B RWST SUCTION MOV-004B FAILS TO CLOSE & $3.3 \mathrm{E}-4$ \\
\hline LPR-XHE-XM-RC & OPERATOR FAILS TO START RECIRC MODE & $2.0 \mathrm{E}-2$ \\
\hline LPR-XHE-XM-RC-H-LD & OPERATOR FAILS TO START RECIRC MODE (DEPENDENCY) & $6.9 \mathrm{E}-2$ \\
\hline MFW-CKV-CC-001 & MFW DISCHARGE CKV-001 TO SG-A FAILS TO OPEN & $9.2 \mathrm{E}-6$ \\
\hline MFW-CKV-CC-002 & MFW DISCHARGE CKV-002 TO SG-B FAILS TO OPEN & $9.2 \mathrm{E}-6$ \\
\hline MFW-CKV-CC-003 & MFW DISCHARGE CKV-003 TO SG-C FAILS TO OPEN & $9.2 \mathrm{E}-6$ \\
\hline MFW-CKV-CC-004 & MFW DISCHARGE CKV-004 SG-D FAILS TO OPEN & $9.2 \mathrm{E}-6$ \\
\hline MFW-CKV-CF-CC & CCF OF MFW CKV TO OPEN & $1.2 \mathrm{E}-7$ \\
\hline MFW-EQUIPMENT-FA & MFW EQUIPMENT AND SUPPORT SYSTEM FAILURES (DEVELOPED EVENT) & $9.6 \mathrm{E}-3$ \\
\hline MFW-XHE-XM-ERROR & OPERATOR FAILS TO START / CONTROL FEED WATER INJECTION & $4.0 \mathrm{E}-2$ \\
\hline MSS-ACT-CF-ACTUATION & COMMON CAUSE FAILURE OF MSS-A \& MSS-B ACUATATION SIGNAL & $5.0 \mathrm{E}-4$ \\
\hline MSS-ARV-CC-001A & MSS PORV 001A FAILS TO OPEN & $4.9 \mathrm{E}-3$ \\
\hline MSS-ARV-CC-001B & MSS PORV 001B FAILS TO OPEN & $4.9 \mathrm{E}-3$ \\
\hline MSS-ARV-CC-001C & MSS PORV 001C FAILS TO OPEN & $4.9 \mathrm{E}-3$ \\
\hline MSS-ARV-CC-001D & MSS PORV 001D FAILS TO OPEN & $4.9 \mathrm{E}-3$ \\
\hline MSS-ARV-CF-PORV & CCF OF SG PORVS TO OPEN & $2.9 \mathrm{E}-5$ \\
\hline MSS-CND-FC-MAIN & MAIN CONDENSER AND CIRC WATER SYSTEMS ARE UNAVAILABLE & $0.0 \mathrm{E}+0$ \\
\hline MSS-ICC-FC-AUTOISO & AUTOMATIC MSIV ISOLATION SIGNAL FAILS & $2.0 \mathrm{E}-3$ \\
\hline MSS-MSV-CF-MSIVS & CCF OF SG STEAM STOP VALVES A/B/C/D TO CLOSE & $5.3 \mathrm{E}-6$ \\
\hline MSS-MSV-OO-MSIVA & FAILURE OF SG A STEAM STOP (ISOLATION) VALVE TO CLOSE & $8.9 \mathrm{E}-4$ \\
\hline MSS-MSV-OO-MSIVB & FAILURE OF SG B STEAM STOP (ISOLATION) VALVE TO CLOSE & $8.9 \mathrm{E}-4$ \\
\hline
\end{tabular}




\section{Generic Pressurized Water Reactor (PWR)}

\begin{tabular}{|c|c|c|}
\hline Basic Event Name & Description & $\begin{array}{l}\text { Calculated } \\
\text { Probability }\end{array}$ \\
\hline MSS-MSV-OO-MSIVC & FAILURE OF SG C STEAM STOP (ISOLATION) VALVE TO CLOSE & $8.9 \mathrm{E}-4$ \\
\hline MSS-MSV-OO-MSIVD & FAILURE OF SG D STEAM STOP (ISOLATION) VALVE TO CLOSE & $8.9 \mathrm{E}-4$ \\
\hline MSS-SVV-OO-003A & MSS SVV003A $\quad$ FAILS TO RECLOSE AFTER OPENING & $1.2 \mathrm{E}-4$ \\
\hline MSS-SVV-OO-003B & MSS-SVV 003B $\quad$ FAILS TO RECLOSE AFTER OPENING & $1.2 \mathrm{E}-4$ \\
\hline MSS-SVV-OO-003C & FAILS TO RECLOSE AFTER OPENING & $1.2 \mathrm{E}-4$ \\
\hline MSS-SVV-OO-003D & FAILS TO RECLOSE AFTER OPENING & $1.2 \mathrm{E}-4$ \\
\hline MSS-SYS-RF-SGTRA & FRACTION OF TIME SGTR IN SG A & $2.5 \mathrm{E}-1$ \\
\hline MSS-SYS-RF-SGTRB & FRACTION OF TIME SGTR IN SG B & $2.5 \mathrm{E}-1$ \\
\hline MSS-SYS-RF-SGTRC & FRACTION OF TIME SGTR IN SG C & $2.5 \mathrm{E}-1$ \\
\hline MSS-SYS-RF-SGTRD & FRACTION OF TIME SGTR IN SG D & $2.5 \mathrm{E}-1$ \\
\hline MSS-TBV-CF-PVS & CONDENSER STEAM DUMP VALVES FAIL TO OPEN ON DEMAND & $5.0 \mathrm{E}-6$ \\
\hline MSS-XHE-XM-MSISOL & OPERATOR FAILS TO ISOLATE STEAM LINE BREAK & $1.0 \mathrm{E}-3$ \\
\hline MSS-XHE-XM-SGISO & OPERATOR FAILS TO ISOLATE FAULTED STEAM GENERATOR & $1.0 \mathrm{E}-3$ \\
\hline OEP-XHE-XL-NR01H & OPERATOR FAILS TO RECOVER OFFSITE POWER IN 1 HOUR & $6.5 \mathrm{E}-1$ \\
\hline OEP-XHE-XL-NR01HGR & OPERATOR FAILS TO RECOVER OFFSITE POWER IN 1 HOUR (GRID-RELATED) & $5.7 \mathrm{E}-1$ \\
\hline OEP-XHE-XL-NR01HPC & OPERATOR FAILS TO RECOVER OFFSITE POWER IN 1 HOUR (PLANT-CENTERED) & $7.0 \mathrm{E}-1$ \\
\hline OEP-XHE-XL-NR01HSC & OPERATOR FAILS TO RECOVER OFFSITE POWER IN 1 HOUR (SWITCHYARD) & $6.4 \mathrm{E}-1$ \\
\hline OEP-XHE-XL-NR01HWR & $\begin{array}{l}\text { OPERATOR FAILS TO RECOVER OFFSITE POWER IN } 1 \text { HOUR (WEATHER- } \\
\text { RELATED) }\end{array}$ & $5.6 \mathrm{E}-1$ \\
\hline OEP-XHE-XL-NR02H & OPERATOR FAILS TO RECOVER OFFSITE POWER IN 2 HOURS & 4.7E-1 \\
\hline OEP-XHE-XL-NR02HGR & OPERATOR FAILS TO RECOVER OFFSITE POWER IN 2 HOURS (GRID-RELATED) & $4.2 \mathrm{E}-1$ \\
\hline OEP-XHE-XL-NR02HPC & $\begin{array}{l}\text { OPERATOR FAILS TO RECOVER OFFSITE POWER IN } 2 \text { HOURS (PLANT- } \\
\text { CENTERED) }\end{array}$ & $5.3 \mathrm{E}-1$ \\
\hline OEP-XHE-XL-NR02HSC & OPERATOR FAILS TO RECOVER OFFSITE POWER IN 2 HOURS (SWITCHYARD) & $4.8 \mathrm{E}-1$ \\
\hline OEP-XHE-XL-NR02HWR & $\begin{array}{l}\text { OPERATOR FAILS TO RECOVER OFFSITE POWER IN } 2 \text { HOURS (WEATHER- } \\
\text { RELATED) }\end{array}$ & $3.8 \mathrm{E}-1$ \\
\hline OEP-XHE-XL-NRO3H & OPERATOR FAILS TO RECOVER OFFSITE POWER IN 3 HOURS & $3.6 \mathrm{E}-1$ \\
\hline OEP-XHE-XL-NR03HGR & OPERATOR FAILS TO RECOVER OFFSITE POWER IN 3 HOURS (GRID-RELATED) & $3.4 \mathrm{E}-1$ \\
\hline OEP-XHE-XL-NR03HPC & $\begin{array}{l}\text { OPERATOR FAILS TO RECOVER OFFSITE POWER IN } 3 \text { HOURS (PLANT- } \\
\text { CENTERED) }\end{array}$ & $4.2 \mathrm{E}-1$ \\
\hline OEP-XHE-XL-NR03HSC & OPERATOR FAILS TO RECOVER OFFSITE POWER IN 3 HOURS (SWITCHYARD) & $3.8 \mathrm{E}-1$ \\
\hline OEP-XHE-XL-NR03HWR & $\begin{array}{l}\text { OPERATOR FAILS TO RECOVER OFFSITE POWER IN } 3 \text { HOURS (WEATHER- } \\
\text { RELATED) }\end{array}$ & $2.7 \mathrm{E}-1$ \\
\hline OEP-XHE-XL-NR04H & OPERATOR FAILS TO RECOVER OFFSITE POWER IN 4 HOURS & $3.0 \mathrm{E}-1$ \\
\hline OEP-XHE-XL-NR04HGR & OPERATOR FAILS TO RECOVER OFFSITE POWER IN 4 HOURS (GRID-RELATED) & $2.9 \mathrm{E}-1$ \\
\hline OEP-XHE-XL-NR04HPC & $\begin{array}{l}\text { OPERATOR FAILS TO RECOVER OFFSITE POWER IN } 4 \text { HOURS (PLANT- } \\
\text { CENTERED) }\end{array}$ & $3.5 \mathrm{E}-1$ \\
\hline OEP-XHE-XL-NR04HSC & OPERATOR FAILS TO RECOVER OFFSITE POWER IN 4 HOURS (SWITCHYARD) & $3.2 \mathrm{E}-1$ \\
\hline OEP-XHE-XL-NR04HWR & $\begin{array}{l}\text { OPERATOR FAILS TO RECOVER OFFSITE POWER IN } 4 \text { HOURS (WEATHER- } \\
\text { RELATED) }\end{array}$ & $2.1 \mathrm{E}-1$ \\
\hline OEP-XHE-XL-NR05H & OPERATOR FAILS TO RECOVER OFFSITE POWER IN 5 HOURS & $2.5 \mathrm{E}-1$ \\
\hline OEP-XHE-XL-NR05HGR & OPERATOR FAILS TO RECOVER OFFSITE POWER IN 5 HOURS (GRID-RELATED) & $2.5 \mathrm{E}-1$ \\
\hline OEP-XHE-XL-NR05HPC & $\begin{array}{l}\text { OPERATOR FAILS TO RECOVER OFFSITE POWER IN } 5 \text { HOURS (PLANT- } \\
\text { CENTERED) }\end{array}$ & $3.1 \mathrm{E}-1$ \\
\hline OEP-XHE-XL-NR05HSC & OPERATOR FAILS TO RECOVER OFFSITE POWER IN 5 HOURS (SWITCHYARD) & $2.8 \mathrm{E}-1$ \\
\hline OEP-XHE-XL-NR05HWR & $\begin{array}{l}\text { OPERATOR FAILS TO RECOVER OFFSITE POWER IN } 5 \text { HOURS (WEATHER- } \\
\text { RELATED) }\end{array}$ & $1.7 \mathrm{E}-1$ \\
\hline OEP-XHE-XL-NR06H & OPERATOR FAILS TO RECOVER OFFSITE POWER IN 6 HOURS & $2.2 \mathrm{E}-1$ \\
\hline OEP-XHE-XL-NR06HGR & OPERATOR FAILS TO RECOVER OFFSITE POWER IN 6 HOURS (GRID-RELATED) & $2.3 \mathrm{E}-1$ \\
\hline OEP-XHE-XL-NR06HPC & $\begin{array}{l}\text { OPERATOR FAILS TO RECOVER OFFSITE POWER IN } 6 \text { HOURS (PLANT- } \\
\text { CENTERED) }\end{array}$ & $2.7 \mathrm{E}-1$ \\
\hline OEP-XHE-XL-NR06HSC & OPERATOR FAILS TO RECOVER OFFSITE POWER IN 6 HOURS (SWITCHYARD) & $2.4 \mathrm{E}-1$ \\
\hline OEP-XHE-XL-NR06HWR & $\begin{array}{l}\text { OPERATOR FAILS TO RECOVER OFFSITE POWER IN } 6 \text { HOURS (WEATHER- } \\
\text { RELATED) }\end{array}$ & $1.4 \mathrm{E}-1$ \\
\hline OEP-XHE-XL-NR07H & OPERATOR FAILS TO RECOVER OFFSITE POWER IN 7 HOURS & $1.9 \mathrm{E}-1$ \\
\hline OEP-XHE-XL-NR07HGR & OPERATOR FAILS TO RECOVER OFFSITE POWER IN 7 HOURS (GRID-RELATED) & $2.0 \mathrm{E}-1$ \\
\hline OEP-XHE-XL-NR07HPC & $\begin{array}{l}\text { OPERATOR FAILS TO RECOVER OFFSITE POWER IN } 7 \text { HOURS (PLANT- } \\
\text { CENTERED) }\end{array}$ & $2.4 \mathrm{E}-1$ \\
\hline
\end{tabular}




\section{Generic Pressurized Water Reactor (PWR)}

\begin{tabular}{|c|c|c|}
\hline Basic Event Name & Description & $\begin{array}{l}\text { Calculated } \\
\text { Probability }\end{array}$ \\
\hline OEP-XHE-XL-NR07HSC & OPERATOR FAILS TO RECOVER OFFSITE POWER IN 7 HOURS (SWITCHYARD) & $2.2 \mathrm{E}-1$ \\
\hline OEP-XHE-XL-NR07HWR & $\begin{array}{l}\text { OPERATOR FAILS TO RECOVER OFFSITE POWER IN } 7 \text { HOURS (WEATHER- } \\
\text { RELATED) }\end{array}$ & $1.1 \mathrm{E}-1$ \\
\hline OEP-XHE-XL-NR08H & OPERATOR FAILS TO RECOVER OFFSITE POWER IN 8 HOURS & $1.7 \mathrm{E}-1$ \\
\hline OEP-XHE-XL-NR08HGR & OPERATOR FAILS TO RECOVER OFFSITE POWER IN 8 HOURS (GRID-RELATED) & $1.9 \mathrm{E}-1$ \\
\hline OEP-XHE-XL-NR08HPC & $\begin{array}{l}\text { OPERATOR FAILS TO RECOVER OFFSITE POWER IN } 8 \text { HOURS (PLANT- } \\
\text { CENTERED) }\end{array}$ & $2.2 \mathrm{E}-1$ \\
\hline OEP-XHE-XL-NR08HSC & OPERATOR FAILS TO RECOVER OFFSITE POWER IN 8 HOURS (SWITCHYARD) & $2.0 \mathrm{E}-1$ \\
\hline OEP-XHE-XL-NR08HWR & $\begin{array}{l}\text { OPERATOR FAILS TO RECOVER OFFSITE POWER IN } 8 \text { HOURS (WEATHER- } \\
\text { RELATED) }\end{array}$ & $9.6 \mathrm{E}-2$ \\
\hline OEP-XHE-XL-NR09H & OPERATOR FAILS TO RECOVER OFFSITE POWER IN 9 HOURS & $1.5 \mathrm{E}-1$ \\
\hline OEP-XHE-XL-NR09HGR & OPERATOR FAILS TO RECOVER OFFSITE POWER IN 9 HOURS (GRID-RELATED) & 1.7E-1 \\
\hline OEP-XHE-XL-NR09HPC & $\begin{array}{l}\text { OPERATOR FAILS TO RECOVER OFFSITE POWER IN } 9 \text { HOURS (PLANT- } \\
\text { CENTERED) }\end{array}$ & $2.0 \mathrm{E}-1$ \\
\hline OEP-XHE-XL-NRO9HSC & OPERATOR FAILS TO RECOVER OFFSITE POWER IN 9 HOURS (SWITCHYARD) & $1.8 \mathrm{E}-1$ \\
\hline OEP-XHE-XL-NR09HWR & $\begin{array}{l}\text { OPERATOR FAILS TO RECOVER OFFSITE POWER IN } 9 \text { HOURS (WEATHER- } \\
\text { RELATED) }\end{array}$ & $8.2 \mathrm{E}-2$ \\
\hline OEP-XHE-XL-NR10H & OPERATOR FAILS TO RECOVER OFFSITE POWER IN 10 HOURS & $1.4 \mathrm{E}-1$ \\
\hline OEP-XHE-XL-NR10HGR & OPERATOR FAILS TO RECOVER OFFSITE POWER IN 10 HOURS (GRID-RELATED) & $1.6 \mathrm{E}-1$ \\
\hline OEP-XHE-XL-NR10HPC & $\begin{array}{l}\text { OPERATOR FAILS TO RECOVER OFFSITE POWER IN } 10 \text { HOURS (PLANT- } \\
\text { CENTERED) }\end{array}$ & $1.8 \mathrm{E}-1$ \\
\hline OEP-XHE-XL-NR10HSC & OPERATOR FAILS TO RECOVER OFFSITE POWER IN 10 HOURS (SWITCHYARD) & 1.7E-1 \\
\hline OEP-XHE-XL-NR10HWR & $\begin{array}{l}\text { OPERATOR FAILS TO RECOVER OFFSITE POWER IN } 10 \text { HOURS (WEATHER- } \\
\text { RELATED) }\end{array}$ & $7.1 \mathrm{E}-2$ \\
\hline OEP-XHE-XL-NR11H & OPERATOR FAILS TO RECOVER OFFSITE POWER IN 11 HOURS & $1.3 \mathrm{E}-1$ \\
\hline OEP-XHE-XL-NR11HGR & OPERATOR FAILS TO RECOVER OFFSITE POWER IN 11 HOURS (GRID-RELATED) & $1.5 \mathrm{E}-1$ \\
\hline OEP-XHE-XL-NR11HPC & $\begin{array}{l}\text { OPERATOR FAILS TO RECOVER OFFSITE POWER IN } 11 \text { HOURS (PLANT- } \\
\text { CENTERED) }\end{array}$ & $1.7 \mathrm{E}-1$ \\
\hline OEP-XHE-XL-NR11HSC & OPERATOR FAILS TO RECOVER OFFSITE POWER IN 11 HOURS (SWITCHYARD) & $1.6 \mathrm{E}-1$ \\
\hline OEP-XHE-XL-NR11HWR & $\begin{array}{l}\text { OPERATOR FAILS TO RECOVER OFFSITE POWER IN } 11 \text { HOURS (WEATHER- } \\
\text { RELATED) }\end{array}$ & $6.2 \mathrm{E}-2$ \\
\hline OEP-XHE-XL-NR12H & OPERATOR FAILS TO RECOVER OFFSITE POWER IN 12 HOURS & $1.2 \mathrm{E}-1$ \\
\hline OEP-XHE-XL-NR12HGR & OPERATOR FAILS TO RECOVER OFFSITE POWER IN 12 HOURS (GRID-RELATED) & $1.4 \mathrm{E}-1$ \\
\hline OEP-XHE-XL-NR12HPC & $\begin{array}{l}\text { OPERATOR FAILS TO RECOVER OFFSITE POWER IN } 12 \text { HOURS (PLANT- } \\
\text { CENTERED) }\end{array}$ & $1.6 \mathrm{E}-1$ \\
\hline OEP-XHE-XL-NR12HSC & OPERATOR FAILS TO RECOVER OFFSITE POWER IN 12 HOURS (SWITCHYARD) & $1.5 \mathrm{E}-1$ \\
\hline OEP-XHE-XL-NR12HWR & $\begin{array}{l}\text { OPERATOR FAILS TO RECOVER OFFSITE POWER IN } 12 \text { HOURS (WEATHER- } \\
\text { RELATED) }\end{array}$ & $5.5 \mathrm{E}-2$ \\
\hline OEP-XHE-XL-NR13H & OPERATOR FAILS TO RECOVER OFFSITE POWER IN 13 HOURS & $1.1 \mathrm{E}-1$ \\
\hline OEP-XHE-XL-NR13HGR & OPERATOR FAILS TO RECOVER OFFSITE POWER IN 13 HOURS (GRID-RELATED) & $1.3 \mathrm{E}-1$ \\
\hline OEP-XHE-XL-NR13HPC & $\begin{array}{l}\text { OPERATOR FAILS TO RECOVER OFFSITE POWER IN } 13 \text { HOURS (PLANT- } \\
\text { CENTERED) }\end{array}$ & $1.5 \mathrm{E}-1$ \\
\hline OEP-XHE-XL-NR13HSC & OPERATOR FAILS TO RECOVER OFFSITE POWER IN 13 HOURS (SWITCHYARD) & $1.4 \mathrm{E}-1$ \\
\hline OEP-XHE-XL-NR13HWR & $\begin{array}{l}\text { OPERATOR FAILS TO RECOVER OFFSITE POWER IN } 13 \text { HOURS (WEATHER- } \\
\text { RELATED) }\end{array}$ & $4.8 \mathrm{E}-2$ \\
\hline OEP-XHE-XL-NR14H & OPERATOR FAILS TO RECOVER OFFSITE POWER IN 14 HOURS & $1.0 \mathrm{E}-1$ \\
\hline OEP-XHE-XL-NR14HGR & OPERATOR FAILS TO RECOVER OFFSITE POWER IN 14 HOURS (GRID-RELATED) & $1.3 \mathrm{E}-1$ \\
\hline OEP-XHE-XL-NR14HPC & $\begin{array}{l}\text { OPERATOR FAILS TO RECOVER OFFSITE POWER IN } 14 \text { HOURS (PLANT- } \\
\text { CENTERED) }\end{array}$ & $1.4 \mathrm{E}-1$ \\
\hline OEP-XHE-XL-NR14HSC & OPERATOR FAILS TO RECOVER OFFSITE POWER IN 14 HOURS (SWITCHYARD) & $1.3 \mathrm{E}-1$ \\
\hline OEP-XHE-XL-NR14HWR & $\begin{array}{l}\text { OPERATOR FAILS TO RECOVER OFFSITE POWER IN } 14 \text { HOURS (WEATHER- } \\
\text { RELATED) }\end{array}$ & $4.3 \mathrm{E}-2$ \\
\hline OEP-XHE-XL-NR15H & OPERATOR FAILS TO RECOVER OFFSITE POWER IN 15 HOURS & $9.3 \mathrm{E}-2$ \\
\hline OEP-XHE-XL-NR15HGR & OPERATOR FAILS TO RECOVER OFFSITE POWER IN 15 HOURS (GRID-RELATED) & $1.2 \mathrm{E}-1$ \\
\hline OEP-XHE-XL-NR15HPC & $\begin{array}{l}\text { OPERATOR FAILS TO RECOVER OFFSITE POWER IN } 15 \text { HOURS (PLANT- } \\
\text { CENTERED) }\end{array}$ & $1.3 \mathrm{E}-1$ \\
\hline OEP-XHE-XL-NR15HSC & OPERATOR FAILS TO RECOVER OFFSITE POWER IN 15 HOURS (SWITCHYARD) & $1.2 \mathrm{E}-1$ \\
\hline OEP-XHE-XL-NR15HWR & $\begin{array}{l}\text { OPERATOR FAILS TO RECOVER OFFSITE POWER IN } 15 \text { HOURS (WEATHER- } \\
\text { RELATED) }\end{array}$ & $3.9 \mathrm{E}-2$ \\
\hline OEP-XHE-XL-NR16H & OPERATOR FAILS TO RECOVER OFFSITE POWER IN 16 HOURS & $8.8 \mathrm{E}-2$ \\
\hline OEP-XHE-XL-NR16HGR & OPERATOR FAILS TO RECOVER OFFSITE POWER IN 16 HOURS (GRID-RELATED) & $1.2 \mathrm{E}-1$ \\
\hline
\end{tabular}




\section{Generic Pressurized Water Reactor (PWR)}

\begin{tabular}{|c|c|c|}
\hline Basic Event Name & Description & $\begin{array}{l}\text { Calculated } \\
\text { Probability }\end{array}$ \\
\hline OEP-XHE-XL-NR16HPC & $\begin{array}{l}\text { OPERATOR FAILS TO RECOVER OFFSITE POWER IN } 16 \text { HOURS (PLANT- } \\
\text { CENTERED) }\end{array}$ & $1.3 \mathrm{E}-1$ \\
\hline OEP-XHE-XL-NR16HSC & OPERATOR FAILS TO RECOVER OFFSITE POWER IN 16 HOURS (SWITCHYARD) & $1.2 \mathrm{E}-1$ \\
\hline OEP-XHE-XL-NR16HWR & $\begin{array}{l}\text { OPERATOR FAILS TO RECOVER OFFSITE POWER IN } 16 \text { HOURS (WEATHER- } \\
\text { RELATED) }\end{array}$ & $3.5 \mathrm{E}-2$ \\
\hline OEP-XHE-XL-NR17H & OPERATOR FAILS TO RECOVER OFFSITE POWER IN 17 HOURS & $8.3 \mathrm{E}-2$ \\
\hline OEP-XHE-XL-NR17HGR & OPERATOR FAILS TO RECOVER OFFSITE POWER IN 17 HOURS (GRID-RELATED) & $1.1 \mathrm{E}-1$ \\
\hline OEP-XHE-XL-NR17HPC & $\begin{array}{l}\text { OPERATOR FAILS TO RECOVER OFFSITE POWER IN } 17 \text { HOURS (PLANT- } \\
\text { CENTERED) }\end{array}$ & $1.2 \mathrm{E}-1$ \\
\hline OEP-XHE-XL-NR17HSC & OPERATOR FAILS TO RECOVER OFFSITE POWER IN 17 HOURS (SWITCHYARD) & 1.1E-1 \\
\hline OEP-XHE-XL-NR17HWR & $\begin{array}{l}\text { OPERATOR FAILS TO RECOVER OFFSITE POWER IN } 17 \text { HOURS (WEATHER- } \\
\text { RELATED) }\end{array}$ & $3.2 \mathrm{E}-2$ \\
\hline OEP-XHE-XL-NR18H & OPERATOR FAILS TO RECOVER OFFSITE POWER IN 18 HOURS & $7.8 \mathrm{E}-2$ \\
\hline OEP-XHE-XL-NR18HGR & OPERATOR FAILS TO RECOVER OFFSITE POWER IN 18 HOURS (GRID-RELATED) & $1.1 \mathrm{E}-1$ \\
\hline OEP-XHE-XL-NR18HPC & $\begin{array}{l}\text { OPERATOR FAILS TO RECOVER OFFSITE POWER IN } 18 \text { HOURS (PLANT- } \\
\text { CENTERED) }\end{array}$ & $1.1 \mathrm{E}-1$ \\
\hline OEP-XHE-XL-NR18HSC & OPERATOR FAILS TO RECOVER OFFSITE POWER IN 18 HOURS (SWITCHYARD) & $1.1 \mathrm{E}-1$ \\
\hline OEP-XHE-XL-NR18HWR & $\begin{array}{l}\text { OPERATOR FAILS TO RECOVER OFFSITE POWER IN } 18 \text { HOURS (WEATHER- } \\
\text { RELATED) }\end{array}$ & $2.9 \mathrm{E}-2$ \\
\hline OEP-XHE-XL-NR19H & OPERATOR FAILS TO RECOVER OFFSITE POWER IN 19 HOURS & $7.4 \mathrm{E}-2$ \\
\hline OEP-XHE-XL-NR19HGR & OPERATOR FAILS TO RECOVER OFFSITE POWER IN 19 HOURS (GRID-RELATED) & $1.0 \mathrm{E}-1$ \\
\hline OEP-XHE-XL-NR19HPC & $\begin{array}{l}\text { OPERATOR FAILS TO RECOVER OFFSITE POWER IN } 19 \text { HOURS (PLANT- } \\
\text { CENTERED) }\end{array}$ & $1.1 \mathrm{E}-1$ \\
\hline OEP-XHE-XL-NR19HSC & OPERATOR FAILS TO RECOVER OFFSITE POWER IN 19 HOURS (SWITCHYARD) & $1.0 \mathrm{E}-1$ \\
\hline OEP-XHE-XL-NR19HWR & $\begin{array}{l}\text { OPERATOR FAILS TO RECOVER OFFSITE POWER IN } 19 \text { HOURS (WEATHER- } \\
\text { RELATED) }\end{array}$ & $2.6 \mathrm{E}-2$ \\
\hline OEP-XHE-XL-NR2OH & OPERATOR FAILS TO RECOVER OFFSITE POWER IN 20 HOURS & $7.1 \mathrm{E}-2$ \\
\hline OEP-XHE-XL-NR2OHGR & OPERATOR FAILS TO RECOVER OFFSITE POWER IN 20 HOURS (GRID-RELATED) & $9.8 \mathrm{E}-2$ \\
\hline OEP-XHE-XL-NR2OHPC & $\begin{array}{l}\text { OPERATOR FAILS TO RECOVER OFFSITE POWER IN } 20 \text { HOURS (PLANT- } \\
\text { CENTERED) }\end{array}$ & $1.0 \mathrm{E}-1$ \\
\hline OEP-XHE-XL-NR2OHSC & OPERATOR FAILS TO RECOVER OFFSITE POWER IN 20 HOURS (SWITCHYARD) & $9.8 \mathrm{E}-2$ \\
\hline OEP-XHE-XL-NR2OHWR & $\begin{array}{l}\text { OPERATOR FAILS TO RECOVER OFFSITE POWER IN } 20 \text { HOURS (WEATHER- } \\
\text { RELATED) }\end{array}$ & $2.4 \mathrm{E}-2$ \\
\hline OEP-XHE-XL-NR21H & OPERATOR FAILS TO RECOVER OFFSITE POWER IN 21 HOURS & $6.7 \mathrm{E}-2$ \\
\hline OEP-XHE-XL-NR21HGR & OPERATOR FAILS TO RECOVER OFFSITE POWER IN 21 HOURS (GRID-RELATED) & $9.4 \mathrm{E}-2$ \\
\hline OEP-XHE-XL-NR21HPC & $\begin{array}{l}\text { OPERATOR FAILS TO RECOVER OFFSITE POWER IN } 21 \text { HOURS (PLANT- } \\
\text { CENTERED) }\end{array}$ & $1.0 \mathrm{E}-1$ \\
\hline OEP-XHE-XL-NR21HSC & OPERATOR FAILS TO RECOVER OFFSITE POWER IN 21 HOURS (SWITCHYARD) & $9.4 \mathrm{E}-2$ \\
\hline OEP-XHE-XL-NR21HWR & $\begin{array}{l}\text { OPERATOR FAILS TO RECOVER OFFSITE POWER IN } 21 \text { HOURS (WEATHER- } \\
\text { RELATED) }\end{array}$ & $2.2 \mathrm{E}-2$ \\
\hline OEP-XHE-XL-NR22H & OPERATOR FAILS TO RECOVER OFFSITE POWER IN 22 HOURS & $6.4 \mathrm{E}-2$ \\
\hline OEP-XHE-XL-NR22HGR & OPERATOR FAILS TO RECOVER OFFSITE POWER IN 22 HOURS (GRID-RELATED) & $9.1 \mathrm{E}-2$ \\
\hline OEP-XHE-XL-NR22HPC & $\begin{array}{l}\text { OPERATOR FAILS TO RECOVER OFFSITE POWER IN } 22 \text { HOURS (PLANT- } \\
\text { CENTERED) }\end{array}$ & $9.6 \mathrm{E}-2$ \\
\hline OEP-XHE-XL-NR22HSC & OPERATOR FAILS TO RECOVER OFFSITE POWER IN 22 HOURS (SWITCHYARD) & $9.1 \mathrm{E}-2$ \\
\hline OEP-XHE-XL-NR22HWR & $\begin{array}{l}\text { OPERATOR FAILS TO RECOVER OFFSITE POWER IN } 22 \text { HOURS (WEATHER- } \\
\text { RELATED) }\end{array}$ & $2.1 \mathrm{E}-2$ \\
\hline OEP-XHE-XL-NR23H & OPERATOR FAILS TO RECOVER OFFSITE POWER IN 23 HOURS & $6.2 \mathrm{E}-2$ \\
\hline OEP-XHE-XL-NR23HGR & OPERATOR FAILS TO RECOVER OFFSITE POWER IN 23 HOURS (GRID-RELATED) & $8.8 \mathrm{E}-2$ \\
\hline OEP-XHE-XL-NR23HPC & $\begin{array}{l}\text { OPERATOR FAILS TO RECOVER OFFSITE POWER IN } 23 \text { HOURS (PLANT- } \\
\text { CENTERED) }\end{array}$ & $9.3 \mathrm{E}-2$ \\
\hline OEP-XHE-XL-NR23HSC & OPERATOR FAILS TO RECOVER OFFSITE POWER IN 23 HOURS (SWITCHYARD) & 8.7E-2 \\
\hline OEP-XHE-XL-NR23HWR & $\begin{array}{l}\text { OPERATOR FAILS TO RECOVER OFFSITE POWER IN } 23 \text { HOURS (WEATHER- } \\
\text { RELATED) }\end{array}$ & $1.9 \mathrm{E}-2$ \\
\hline OEP-XHE-XL-NR24H & OPERATOR FAILS TO RECOVER OFFSITE POWER IN 24 HOURS & $5.9 \mathrm{E}-2$ \\
\hline OEP-XHE-XL-NR24H8 & OPERATOR FAILS TO RECOVER OFFSITE POWER IN 24 HOURS (FAIL @ 8) & $3.5 \mathrm{E}-1$ \\
\hline OEP-XHE-XL-NR24HGR & OPERATOR FAILS TO RECOVER OFFSITE POWER IN 24 HOURS (GRID-RELATED) & $8.5 \mathrm{E}-2$ \\
\hline OEP-XHE-XL-NR24HPC & $\begin{array}{l}\text { OPERATOR FAILS TO RECOVER OFFSITE POWER IN } 24 \text { HOURS (PLANT- } \\
\text { CENTERED) }\end{array}$ & $9.0 \mathrm{E}-2$ \\
\hline OEP-XHE-XL-NR24HSC & OPERATOR FAILS TO RECOVER OFFSITE POWER IN 24 HOURS (SWITCHYARD) & $8.5 \mathrm{E}-2$ \\
\hline OEP-XHE-XL-NR24HWR & OPERATOR FAILS TO RECOVER OFFSITE POWER IN 24 HOURS (WEATHER- & $1.8 \mathrm{E}-2$ \\
\hline
\end{tabular}




\section{Generic Pressurized Water Reactor (PWR)}

\begin{tabular}{|c|c|c|}
\hline Basic Event Name & Description & $\begin{array}{l}\text { Calculated } \\
\text { Probability }\end{array}$ \\
\hline & RELATED) & \\
\hline OEP-XHE-XL-NR3OM & OPERATOR FAILS TO RECOVER OFFSITE POWER IN 30 MINUTES & $7.9 \mathrm{E}-1$ \\
\hline OEP-XHE-XL-NR3OMGR & $\begin{array}{l}\text { OPERATOR FAILS TO RECOVER OFFSITE POWER IN } 30 \text { MINUTES (GRID- } \\
\text { RELATED) }\end{array}$ & $7.2 \mathrm{E}-1$ \\
\hline OEP-XHE-XL-NR3OMPC & $\begin{array}{l}\text { OPERATOR FAILS TO RECOVER OFFSITE POWER IN } 30 \text { MINUTES (PLANT- } \\
\text { CENTERED) }\end{array}$ & 8.3E-1 \\
\hline OEP-XHE-XL-NR3OMSC & OPERATOR FAILS TO RECOVER OFFSITE POWER IN 30 MINUTES (SWITCHYARD) & $7.8 \mathrm{E}-1$ \\
\hline OEP-XHE-XL-NR3OMWR & $\begin{array}{l}\text { OPERATOR FAILS TO RECOVER OFFSITE POWER IN } 30 \text { MINUTES (WEATHER- } \\
\text { RELATED) }\end{array}$ & $7.3 \mathrm{E}-1$ \\
\hline OEP-XHE-XL-NR72H & OPERATOR FAILS TO RECOVER OFFSITE POWER IN 72 HOURS & $2.0 \mathrm{E}-2$ \\
\hline OEP-XHE-XL-NR72H8 & OPERATOR FAILS TO RECOVER OFFSITE POWER IN 72 HOURS (FAIL @ 8) & $6.8 \mathrm{E}-2$ \\
\hline OEP-XHE-XL-NR90M & OPERATOR FAILS TO RECOVER OFFSITE POWER IN 90 MINUTES & $5.4 \mathrm{E}-1$ \\
\hline OEP-XHE-XL-NR9OMGR & $\begin{array}{l}\text { OPERATOR FAILS TO RECOVER OFFSITE POWER IN } 90 \text { MINUTES (GRID- } \\
\text { RELATED) }\end{array}$ & $4.8 \mathrm{E}-1$ \\
\hline OEP-XHE-XL-NR9OMPC & $\begin{array}{l}\text { OPERATOR FAILS TO RECOVER OFFSITE POWER IN } 90 \text { MINUTES (PLANT- } \\
\text { CENTERED) }\end{array}$ & $6.0 \mathrm{E}-1$ \\
\hline OEP-XHE-XL-NR9OMSC & OPERATOR FAILS TO RECOVER OFFSITE POWER IN 90 MINUTES (SWITCHYARD) & $5.5 \mathrm{E}-1$ \\
\hline OEP-XHE-XL-NR9OMWR & $\begin{array}{l}\text { OPERATOR FAILS TO RECOVER OFFSITE POWER IN } 90 \text { MINUTES (WEATHER- } \\
\text { RELATED) }\end{array}$ & $4.5 \mathrm{E}-1$ \\
\hline OEP-XHE-XX-NR01HGRO & Convolution Factor for CCF-OPR (01H-GR) & $2.8 \mathrm{E}-1$ \\
\hline OEP-XHE-XX-NR01HGR1 & Convolution Factor for 1FTR-OPR (01H-GR) & $2.8 \mathrm{E}-1$ \\
\hline OEP-XHE-XX-NR01HGR2 & Convolution Factor for 2FTR-OPR (01H-GR) & $2.1 \mathrm{E}-1$ \\
\hline OEP-XHE-XX-NR01HGR3 & Convolution Factor for 3FTR-OPR (01H-GR) & $1.8 \mathrm{E}-1$ \\
\hline OEP-XHE-XX-NR01HPCO & Convolution Factor for CCF-OPR (01H-PC) & $2.6 \mathrm{E}-1$ \\
\hline OEP-XHE-XX-NR01HPC1 & Convolution Factor for 1FTR-OPR (01H-PC) & $2.6 \mathrm{E}-1$ \\
\hline OEP-XHE-XX-NR01HPC2 & Convolution Factor for 2FTR-OPR (01H-PC) & $1.9 \mathrm{E}-1$ \\
\hline OEP-XHE-XX-NR01HPC3 & Convolution Factor for 3FTR-OPR (01H-PC) & $1.6 \mathrm{E}-1$ \\
\hline OEP-XHE-XX-NR01HSCO & Convolution Factor for CCF-OPR (01H-SC) & $2.6 \mathrm{E}-1$ \\
\hline OEP-XHE-XX-NR01HSC1 & Convolution Factor for 1FTR-OPR (01H-SC) & $2.6 \mathrm{E}-1$ \\
\hline OEP-XHE-XX-NR01HSC2 & Convolution Factor for 2FTR-OPR (01H-SC) & $1.9 \mathrm{E}-1$ \\
\hline OEP-XHE-XX-NR01HSC3 & Convolution Factor for 3FTR-OPR (01H-SC) & $1.7 \mathrm{E}-1$ \\
\hline OEP-XHE-XX-NR01HWRO & Convolution Factor for CCF-OPR (01H-WR) & $1.4 \mathrm{E}-1$ \\
\hline OEP-XHE-XX-NR01HWR1 & Convolution Factor for 1FTR-OPR (01H-WR) & $1.4 \mathrm{E}-1$ \\
\hline OEP-XHE-XX-NR01HWR2 & Convolution Factor for 2FTR-OPR (01H-WR) & $7.4 \mathrm{E}-2$ \\
\hline OEP-XHE-XX-NR01HWR3 & Convolution Factor for 3FTR-OPR (01H-WR) & $5.4 \mathrm{E}-2$ \\
\hline OEP-XHE-XX-NRO2HGRO & Convolution Factor for CCF-OPR (02H-GR) & $3.5 \mathrm{E}-1$ \\
\hline OEP-XHE-XX-NR02HGR1 & Convolution Factor for 1FTR-OPR (02H-GR) & $3.5 \mathrm{E}-1$ \\
\hline OEP-XHE-XX-NR02HGR2 & Convolution Factor for 2FTR-OPR (02H-GR) & $2.7 \mathrm{E}-1$ \\
\hline OEP-XHE-XX-NR02HGR3 & Convolution Factor for 3FTR-OPR (02H-GR) & $2.4 \mathrm{E}-1$ \\
\hline OEP-XHE-XX-NR02HPCO & Convolution Factor for CCF-OPR (02H-PC) & $3.2 \mathrm{E}-1$ \\
\hline OEP-XHE-XX-NR02HPC1 & Convolution Factor for 1FTR-OPR (02H-PC) & $3.2 \mathrm{E}-1$ \\
\hline OEP-XHE-XX-NR02HPC2 & Convolution Factor for 2FTR-OPR (02H-PC) & $2.3 \mathrm{E}-1$ \\
\hline OEP-XHE-XX-NR02HPC3 & Convolution Factor for 3FTR-OPR (02H-PC) & $2.1 \mathrm{E}-1$ \\
\hline OEP-XHE-XX-NR02HSC0 & Convolution Factor for CCF-OPR (02H-SC) & $3.2 \mathrm{E}-1$ \\
\hline OEP-XHE-XX-NR02HSC1 & Convolution Factor for 1FTR-OPR (02H-SC) & $3.2 \mathrm{E}-1$ \\
\hline OEP-XHE-XX-NR02HSC2 & Convolution Factor for 2FTR-OPR (02H-SC) & $2.4 \mathrm{E}-1$ \\
\hline OEP-XHE-XX-NR02HSC3 & Convolution Factor for 3FTR-OPR (02H-SC) & $2.1 \mathrm{E}-1$ \\
\hline OEP-XHE-XX-NR02HWRO & Convolution Factor for CCF-OPR (02H-WR) & $1.8 \mathrm{E}-1$ \\
\hline OEP-XHE-XX-NR02HWR1 & Convolution Factor for 1FTR-OPR (02H-WR) & $1.8 \mathrm{E}-1$ \\
\hline OEP-XHE-XX-NR02HWR2 & Convolution Factor for 2FTR-OPR (02H-WR) & $9.7 \mathrm{E}-2$ \\
\hline OEP-XHE-XX-NR02HWR3 & Convolution Factor for 3FTR-OPR (02H-WR) & $7.4 \mathrm{E}-2$ \\
\hline OEP-XHE-XX-NR08HO & Convolution Factor for CCF-OPR (08H-) & $4.8 \mathrm{E}-1$ \\
\hline OEP-XHE-XX-NR08H1 & Convolution Factor for 1FTR-OPR (08H-) & $4.8 \mathrm{E}-1$ \\
\hline OEP-XHE-XX-NR08H2 & Convolution Factor for 2FTR-OPR (08H-) & $4.0 \mathrm{E}-1$ \\
\hline OEP-XHE-XX-NR08HGRO & Convolution Factor for CCF-OPR (08H-GR) & $5.7 \mathrm{E}-1$ \\
\hline
\end{tabular}




\section{Generic Pressurized Water Reactor (PWR)}

\begin{tabular}{|c|c|c|}
\hline Basic Event Name & Description & $\begin{array}{l}\text { Calculated } \\
\text { Probability }\end{array}$ \\
\hline OEP-XHE-XX-NR08HGR1 & Convolution Factor for 1FTR-OPR (08H-GR) & $5.7 \mathrm{E}-1$ \\
\hline OEP-XHE-XX-NR08HGR2 & Convolution Factor for 2FTR-OPR (08H-GR) & $5.0 \mathrm{E}-1$ \\
\hline OEP-XHE-XX-NR08HGR3 & Convolution Factor for 3FTR-OPR (08H-GR) & $4.7 \mathrm{E}-1$ \\
\hline OEP-XHE-XX-NR08HPC0 & Convolution Factor for CCF-OPR (08H-PC) & $5.3 \mathrm{E}-1$ \\
\hline OEP-XHE-XX-NR08HPC1 & Convolution Factor for 1FTR-OPR (08H-PC) & $5.3 \mathrm{E}-1$ \\
\hline OEP-XHE-XX-NR08HPC2 & Convolution Factor for 2FTR-OPR (08H-PC) & $4.6 \mathrm{E}-1$ \\
\hline OEP-XHE-XX-NR08HPC3 & Convolution Factor for 3FTR-OPR (08H-PC) & $4.3 \mathrm{E}-1$ \\
\hline OEP-XHE-XX-NR08HSCO & Convolution Factor for CCF-OPR (08H-SC) & $5.4 \mathrm{E}-1$ \\
\hline OEP-XHE-XX-NR08HSC1 & Convolution Factor for 1FTR-OPR (08H-SC) & $5.4 \mathrm{E}-1$ \\
\hline OEP-XHE-XX-NR08HSC2 & Convolution Factor for 2FTR-OPR (08H-SC) & $4.7 \mathrm{E}-1$ \\
\hline OEP-XHE-XX-NR08HSC3 & Convolution Factor for 3FTR-OPR (08H-SC) & $4.4 \mathrm{E}-1$ \\
\hline OEP-XHE-XX-NR08HWRO & Convolution Factor for CCF-OPR (08H-WR) & $3.3 \mathrm{E}-1$ \\
\hline OEP-XHE-XX-NR08HWR1 & Convolution Factor for 1FTR-OPR (08H-WR) & $3.3 \mathrm{E}-1$ \\
\hline OEP-XHE-XX-NR08HWR2 & Convolution Factor for 2FTR-OPR (08H-WR) & $2.3 \mathrm{E}-1$ \\
\hline OEP-XHE-XX-NR08HWR3 & Convolution Factor for 3FTR-OPR (08H-WR) & $2.0 \mathrm{E}-1$ \\
\hline OPR-XHE-FA-ASD & Operator fails to control AFW and plant from ASD panel after MCR evacuation & 8.7E-2 \\
\hline OPR-XHE-XM-RSSDEP & OPERATOR FAILS TO COOLDOWN RCS TO 1720 PSI IN 2 HOURS & $1.0 \mathrm{E}-3$ \\
\hline OPR-XHE-XM-RSSDEP-H-LD & OPERATOR FAILS TO COOLDOWN RCS TO 1720 PSI IN 2 HOURS (DEPENDENCY) & $5.4 \mathrm{E}-2$ \\
\hline PCS-SGT-RP-SLBISGTR & STEAM LINE BREAK INDUCES A STEAM GENERATOR TUBE RUPTURE & $1.0 \mathrm{E}-3$ \\
\hline PORV-PRV-CO-1 & PORVS/SRVS OPEN DURING TRANSIENT & $1.0 \mathrm{E}+0$ \\
\hline PORV-PRV-CO-L & PORVS/SRVS OPEN DURING LOOP & $1.5 \mathrm{E}-1$ \\
\hline PORV-PRV-CO-SBO & PORVS/SRVS OPEN DURING SBO & $3.7 \mathrm{E}-1$ \\
\hline PORV-PRV-CO-TRANS & PORVS/SRVS OPEN DURING TRANSIENT & $3.5 \mathrm{E}-2$ \\
\hline PPR-AOV-CC-001A & PRESSURIZER SPRAY VALVE 001A FAILS TO OPEN & $7.8 \mathrm{E}-4$ \\
\hline PPR-AOV-CC-001B & PRESSURIZER SPRAY VALVE 001B FAILS TO OPEN & $7.8 \mathrm{E}-4$ \\
\hline PPR-AOV-CF-CC & CCF OF SPRAY VALVES TO OPEN & $2.9 \mathrm{E}-5$ \\
\hline PPR-PRV-CC-001 & PRESSURIZER PORV 1 FAILS TO OPEN & $3.2 \mathrm{E}-3$ \\
\hline PPR-PRV-CC-002 & PRESSURIZER PORV 2 FAILS TO OPEN & $3.2 \mathrm{E}-3$ \\
\hline PPR-PRV-CF-CC & CCF OF PORVS TO OPEN & $4.7 \mathrm{E}-5$ \\
\hline PPR-PRV-OO-001 & PRESSURIZER PORV 1 FAILS TO RECLOSE & $7.3 \mathrm{E}-4$ \\
\hline PPR-PRV-OO-001AL & PRESSURIZER PORV 1 FAILS TO RECLOSE AFTER PASSING LIQUID & $6.3 \mathrm{E}-2$ \\
\hline PPR-PRV-OO-002 & PRESSURIZER PORV 2 FAILS TO RECLOSE & $7.3 \mathrm{E}-4$ \\
\hline PPR-PRV-OO-002AL & PRESSURIZER PORV 2 FAILS TO RECLOSE AFTER PASSING LIQUID & $6.3 \mathrm{E}-2$ \\
\hline PPR-SRV-CC-001 & PRESSURIZER SRV 1 FAILS TO OPEN & $5.2 \mathrm{E}-4$ \\
\hline PPR-SRV-CC-002 & PRESSURIZER SRV 2 FAILS TO OPEN & $5.2 \mathrm{E}-4$ \\
\hline PPR-SRV-CC-003 & PRESSURIZER SRV 3 FAILS TO OPEN & $5.2 \mathrm{E}-4$ \\
\hline PPR-SRV-OO-001 & PRESSURIZER SRV 1 FAILS TO RECLOSE & $7.3 \mathrm{E}-4$ \\
\hline PPR-SRV-OO-001L & PRESSURIZER SRV 1L FAILS TO RECLOSE AFTER PASSING WATER & $1.0 \mathrm{E}-1$ \\
\hline PPR-SRV-OO-002 & PRESSURIZER SRV 2 FAILS TO RECLOSE & $7.3 \mathrm{E}-4$ \\
\hline PPR-SRV-OO-002L & PRESSURIZER SRV 2L FAILS TO RECLOSE AFTER PASSING WATER & $1.0 \mathrm{E}-1$ \\
\hline PPR-SRV-OO-003 & PRESSURIZER SRV 3 FAILS TO RECLOSE & $7.3 \mathrm{E}-4$ \\
\hline PPR-SRV-OO-003L & PRESSURIZER SRV 3L FAILS TO RECLOSE AFTER PASSING WATER & $1.0 \mathrm{E}-1$ \\
\hline PWR-XHE-XM-DEPRCS & OPERATOR FAILS TO DEPRESS RCS/SECONDARY (SSC) & $4.0 \mathrm{E}-3$ \\
\hline RCS-CKV-CC-001 & RCS CKV-001 FAILS TO OPEN & $9.2 \mathrm{E}-6$ \\
\hline RCS-CKV-CC-002 & RCS CKV-002 FAILS TO OPEN & $9.2 \mathrm{E}-6$ \\
\hline RCS-CKV-CC-003 & RCS CKV-003 FAILS TO OPEN & $9.2 \mathrm{E}-6$ \\
\hline RCS-CKV-CC-004 & RCS CKV-004 FAILS TO OPEN & $9.2 \mathrm{E}-6$ \\
\hline RCS-CKV-CF-CC & CCF OF RCS CKV TO OPEN & $1.2 \mathrm{E}-7$ \\
\hline RCS-LOCA-CL1 & LOCA IN COLD LEG 1 & $2.5 \mathrm{E}-1$ \\
\hline RCS-LOCA-CL2 & LOCA IN COLD LEG 2 & $2.5 \mathrm{E}-1$ \\
\hline RCS-LOCA-CL3 & LOCA IN COLD LEG 3 & $2.5 \mathrm{E}-1$ \\
\hline RCS-LOCA-CL4 & LOCA IN COLD LEG 4 & $2.5 \mathrm{E}-1$ \\
\hline
\end{tabular}




\section{Generic Pressurized Water Reactor (PWR)}

\begin{tabular}{|c|c|c|}
\hline Basic Event Name & Description & $\begin{array}{l}\text { Calculated } \\
\text { Probability }\end{array}$ \\
\hline RCS-MDP-LK & RCP SEALS FAIL & $2.1 \mathrm{E}-1$ \\
\hline RCS-MOV-CC-007A & RCS MOV-007A FAILS TO OPEN & $4.2 \mathrm{E}-4$ \\
\hline RCS-MOV-CC-008A & RCS MOV-008A FAILS TO OPEN & $4.2 \mathrm{E}-4$ \\
\hline RCS-MOV-CC-008B & RCS MOV-008B FAILS TO OPEN & $4.2 \mathrm{E}-4$ \\
\hline RCS-MOV-CO-007A & RCS MOV-007A FAILS TO REMAIN CLOSE & $7.9 \mathrm{E}-5$ \\
\hline RCS-MOV-CO-007B & RCS MOV-007B FAILS TO REMAIN CLOSE & $7.9 \mathrm{E}-5$ \\
\hline RCS-MOV-CO-008A & RCS MOV-008A FAILS TO REMAIN CLOSE & $7.9 \mathrm{E}-5$ \\
\hline RCS-MOV-CO-008B & RCS MOV-008B FAILS TO REMAIN CLOSE & $7.9 \mathrm{E}-5$ \\
\hline RCS-PHIN-MODPOOR & MODERATOR TEMP COEFFICIENT NOT ENOUGH NEGATIVE & $1.4 \mathrm{E}-2$ \\
\hline RCS-POWER-HIGH & POWER AT HIGH-LEVEL & $9.0 \mathrm{E}-1$ \\
\hline RCS-RCP-EQ1-LLOCA & LARGE LOCA OCCURS - SEISMIC BIN 1 & $1.0 \mathrm{E}-6$ \\
\hline RCS-RCP-EQ2-LLOCA & LARGE LOCA OCCURS - SEISMIC BIN 2 & $2.1 \mathrm{E}-3$ \\
\hline RCS-RCP-EQ3-LLOCA & LARGE LOCA OCCURS - SEISMIC BIN 3 & $3.8 \mathrm{E}-2$ \\
\hline RCS-RCP-EQ4-LLOCA & LARGE LOCA OCCURS - SEISMIC BIN 4 & $1.7 \mathrm{E}-1$ \\
\hline RCS-RCP-EQ5-LLOCA & LARGE LOCA OCCURS - SEISMIC BIN 5 & 1.7E-1 \\
\hline RCS-RCP-EQ6-LLOCA & LARGE LOCA OCCURS - SEISMIC BIN 6 & $4.4 \mathrm{E}-1$ \\
\hline RCS-SYS-EQ1-LLOCA & LARGE LOCA OCCURS - SEISMIC BIN 1 & $1.5 \mathrm{E}-10$ \\
\hline RCS-SYS-EQ1-SLOCA & SMALL LOCA OCCURS - SEISMIC BIN 1 & $5.1 \mathrm{E}-5$ \\
\hline RCS-SYS-EQ2-LLOCA & LARGE LOCA OCCURS - SEISMIC BIN 2 & $5.5 \mathrm{E}-6$ \\
\hline RCS-SYS-EQ2-SLOCA & SMALL LOCA OCCURS - SEISMIC BIN 2 & $2.3 \mathrm{E}-2$ \\
\hline RCS-SYS-EQ3-LLOCA & LARGE LOCA OCCURS - SEISMIC BIN 3 & $4.5 \mathrm{E}-4$ \\
\hline RCS-SYS-EQ3-SLOCA & SMALL LOCA OCCURS - SEISMIC BIN 3 & $1.8 \mathrm{E}-1$ \\
\hline RCS-SYS-EQ4-LLOCA & LARGE LOCA OCCURS - SEISMIC BIN 4 & $6.2 \mathrm{E}-3$ \\
\hline RCS-SYS-EQ4-SLOCA & SMALL LOCA OCCURS - SEISMIC BIN 4 & $4.6 \mathrm{E}-1$ \\
\hline RCS-SYS-EQ5-LLOCA & LARGE LOCA OCCURS - SEISMIC BIN 5 & $6.2 \mathrm{E}-3$ \\
\hline RCS-SYS-EQ5-SLOCA & SMALL LOCA OCCURS - SEISMIC BIN 5 & 7.7E-1 \\
\hline RCS-SYS-EQ6-LLOCA & LARGE LOCA OCCURS - SEISMIC BIN 6 & $4.6 \mathrm{E}-2$ \\
\hline RCS-SYS-EQ6-SLOCA & SMALL LOCA OCCURS - SEISMIC BIN 6 & $9.8 \mathrm{E}-1$ \\
\hline RCS-XHE-XM-ECA312 & OPERATOR FAILS TO IMPLEMENT SGTR PROCEDURE ECA 3_1/3_2 & $4.0 \mathrm{E}-3$ \\
\hline RCS-XHE-XM-SGTR & OPERATOR FAILS TO IDENTIFY SGTR AND IMPLEMENT PROCEDURES & $1.0 \mathrm{E}-3$ \\
\hline RCS-XHE-XM-TRIP & OPERATOR FAILS TO TRIP RCP AFTER LOSS OF COOLING & $1.0 \mathrm{E}-3$ \\
\hline RHR-EQ3-EQ & SEISMIC FAILURE OF RHR SYSTEM BASIC EVENT BIN 3 & $1.2 \mathrm{E}-1$ \\
\hline RHR-EQ4-EQ & SEISMIC FAILURE OF RHR SYSTEM BASIC EVENT BIN 4 & $5.0 \mathrm{E}-1$ \\
\hline RHR-EQ5-EQ & SEISMIC FAILURE OF RHR SYSTEM BASIC EVENT BIN 5 & $6.9 \mathrm{E}-1$ \\
\hline RHR-HTX-EQ1-BE & RHR HTX FAILURE DUE TO SESIMIC EVENT BIN 1 & $4.9 \mathrm{E}-4$ \\
\hline RHR-HTX-EQ2-BE & RHR HTX FAILURE DUE TO SESIMIC EVENT BIN 2 & $8.1 \mathrm{E}-2$ \\
\hline RHR-HTX-EQ3-BE & RHR HTX FAILURE DUE TO SESIMIC EVENT BIN 3 & $3.8 \mathrm{E}-1$ \\
\hline RHR-HTX-EQ4-BE & RHR HTX FAILURE DUE TO SESIMIC EVENT BIN 4 & $6.9 \mathrm{E}-1$ \\
\hline RHR-HTX-EQ5-BE & RHR HTX FAILURE DUE TO SESIMIC EVENT BIN 5 & $9.1 \mathrm{E}-1$ \\
\hline RHR-HTX-EQ6-BE & RHR HTX FAILURE DUE TO SESIMIC EVENT BIN 6 & $1.0 \mathrm{E}+0$ \\
\hline RHR-MDP-EQ1-BE & RHR MDP FAILURE DUE TO SESIMIC EVENT BIN 1 & $6.5 \mathrm{E}-6$ \\
\hline RHR-MDP-EQ2-BE & RHR MDP FAILURE DUE TO SESIMIC EVENT BIN 2 & $6.9 \mathrm{E}-3$ \\
\hline RHR-MDP-EQ3-BE & RHR MDP FAILURE DUE TO SESIMIC EVENT BIN 3 & $8.3 \mathrm{E}-2$ \\
\hline RHR-MDP-EQ4-BE & RHR MDP FAILURE DUE TO SESIMIC EVENT BIN 4 & $2.9 \mathrm{E}-1$ \\
\hline RHR-MDP-EQ5-BE & RHR MDP FAILURE DUE TO SESIMIC EVENT BIN 5 & $6.0 \mathrm{E}-1$ \\
\hline RHR-MDP-EQ6-BE & RHR MDP FAILURE DUE TO SESIMIC EVENT BIN 6 & $9.4 \mathrm{E}-1$ \\
\hline RPS-BME-CF-RTBAB & CCF OF RTB-A AND RTB-B (MECHANICAL) & $1.6 \mathrm{E}-6$ \\
\hline RPS-CBI-CF-4OF6 & CCF 4 BISTABLES IN 2 OF 3 CHANNELS & $8.2 \mathrm{E}-6$ \\
\hline RPS-CBI-CF-6OF8 & CCF 6 BISTABLES IN 3 OF 4 CHANNELS & $2.7 \mathrm{E}-6$ \\
\hline RPS-CCP-TM-CHA & CH-A IN T\&M & $5.0 \mathrm{E}-3$ \\
\hline RPS-CCX-CF-4OF6 & CCF 4 ANALOG PROCESS LOGIC MODULES IN 2 OF 3 CHANNELS & $6.3 \mathrm{E}-6$ \\
\hline RPS-CCX-CF-6OF8 & CCF 6 ANALOG PROCESS LOGIC MODULES IN 3 OF 4 CHANNELS & $1.8 \mathrm{E}-6$ \\
\hline
\end{tabular}




\section{Generic Pressurized Water Reactor (PWR)}

\begin{tabular}{|c|c|c|}
\hline Basic Event Name & Description & $\begin{array}{l}\text { Calculated } \\
\text { Probability }\end{array}$ \\
\hline RPS-CRM-CF-RCCAS & CCF 10 OR MORE RCCAS FAIL TO DROP & $1.2 \mathrm{E}-6$ \\
\hline RPS-ROD-EQ1-BE & RPS RODS FAILURE DUE TO SESIMIC EVENT BIN 1 & $2.5 \mathrm{E}-6$ \\
\hline RPS-ROD-EQ2-BE & RPS RODS FAILURE DUE TO SESIMIC EVENT BIN 2 & $3.8 \mathrm{E}-3$ \\
\hline RPS-ROD-EQ3-BE & RPS RODS FAILURE DUE TO SESIMIC EVENT BIN 3 & $5.6 \mathrm{E}-2$ \\
\hline RPS-ROD-EQ4-BE & RPS RODS FAILURE DUE TO SESIMIC EVENT BIN 4 & $2.2 \mathrm{E}-1$ \\
\hline RPS-ROD-EQ5-BE & RPS RODS FAILURE DUE TO SESIMIC EVENT BIN 5 & $5.2 \mathrm{E}-1$ \\
\hline RPS-ROD-EQ6-BE & RPS RODS FAILURE DUE TO SESIMIC EVENT BIN 6 & $9.1 \mathrm{E}-1$ \\
\hline RPS-UVL-CF-UVDAB & CCF UV DRIVERS TRAINS A AND B (2 OF 2) & $1.0 \mathrm{E}-5$ \\
\hline RPS-XHE-XM-NSGNL & OPERATOR FAILS TO RESPOND WITH NO RPS SIGNAL PRESENT & $1.2 \mathrm{E}-1$ \\
\hline RPS-XHE-XM-SIGNL & OPERATOR FAILS TO RESPOND WITH RPS SIGNAL PRESENT & $1.0 \mathrm{E}-2$ \\
\hline RWST-EQ1-BE & RWST FAILURE DUE TO SESIMIC EVENT BIN 1 & $1.5 \mathrm{E}-4$ \\
\hline RWST-EQ2-BE & RWST FAILURE DUE TO SESIMIC EVENT BIN 2 & $4.3 \mathrm{E}-2$ \\
\hline RWST-EQ3-BE & RWST FAILURE DUE TO SESIMIC EVENT BIN 3 & $2.6 \mathrm{E}-1$ \\
\hline RWST-EQ4-BE & RWST FAILURE DUE TO SESIMIC EVENT BIN 4 & $5.7 \mathrm{E}-1$ \\
\hline RWST-EQ5-BE & RWST FAILURE DUE TO SESIMIC EVENT BIN 5 & $8.4 \mathrm{E}-1$ \\
\hline RWST-EQ6-BE & RWST FAILURE DUE TO SESIMIC EVENT BIN 6 & $9.9 \mathrm{E}-1$ \\
\hline SGS-EQ1-CD & STEAM GENERATORS FAIL DIRECT CD BIN 1 & $1.5 \mathrm{E}-10$ \\
\hline SGS-EQ2-CD & STEAM GENERATORS FAIL DIRECT CD BIN 2 & $5.5 \mathrm{E}-6$ \\
\hline SGS-EQ3-CD & STEAM GENERATORS FAIL DIRECT CD BIN 3 & $4.5 \mathrm{E}-4$ \\
\hline SGS-EQ4-CD & STEAM GENERATORS FAIL DIRECT CD BIN 4 & $6.2 \mathrm{E}-3$ \\
\hline SGS-EQ5-CD & STEAM GENERATORS FAIL DIRECT CD BIN 5 & $4.6 \mathrm{E}-2$ \\
\hline SGS-EQ6-CD & STEAM GENERATORS FAIL DIRECT CD BIN 6 & $3.5 \mathrm{E}-1$ \\
\hline SGS-EQ7-CD & STEAM GENERATORS FAIL DIRECT CD BIN 7 & $6.7 \mathrm{E}-1$ \\
\hline SIS-CKV-CC-001 & SIS RCS CKV-001 FAILS TO OPEN & $9.2 \mathrm{E}-6$ \\
\hline SIS-CKV-CC-002 & SIS RCS CKV-002 FAILS TO OPEN & $9.2 \mathrm{E}-6$ \\
\hline SIS-CKV-CC-003 & SIS RCS CKV-003 FAILS TO OPEN & $9.2 \mathrm{E}-6$ \\
\hline SIS-CKV-CC-003A & SIS SUCTION CKV-003A FAILS TO OPEN & $9.2 \mathrm{E}-6$ \\
\hline SIS-CKV-CC-003B & SIS SUCTION CKV-003B FAILS TO OPEN & $9.2 \mathrm{E}-6$ \\
\hline SIS-CKV-CC-004 & SIS RCS CKV-004 FAILS TO OPEN & $9.2 \mathrm{E}-6$ \\
\hline SIS-CKV-CC-004A & SIS DISCHARGE CKV-004A FAILS TO OPEN & $9.2 \mathrm{E}-6$ \\
\hline SIS-CKV-CC-004B & SIS DISCHARGE CKV-004B FAILS TO OPEN & $9.2 \mathrm{E}-6$ \\
\hline SIS-CKV-CF-DIS004 & CCF OF SIS DISCHARGE CKV TO OPEN & 1.9E-7 \\
\hline SIS-CKV-CF-RCSCL & CCF OF SIS COLD LEG DISCHARGE CKVS & $1.2 \mathrm{E}-7$ \\
\hline SIS-CKV-CF-SUC003 & CCF OF SIS SUCTION CKV TO OPEN & $1.9 \mathrm{E}-7$ \\
\hline SIS-MDP-CF-FR & CCF OF SIS MDP TO RUN & $1.2 \mathrm{E}-5$ \\
\hline SIS-MDP-CF-FS & CCF OF SIS MDP TO START & $1.3 \mathrm{E}-5$ \\
\hline SIS-MDP-EQ1-BE & SIS MDP FAILURE DUE TO SESIMIC EVENT BIN 1 & $6.5 \mathrm{E}-6$ \\
\hline SIS-MDP-EQ2-BE & SIS MDP FAILURE DUE TO SESIMIC EVENT BIN 2 & $6.9 \mathrm{E}-3$ \\
\hline SIS-MDP-EQ3-BE & SIS MDP FAILURE DUE TO SESIMIC EVENT BIN 3 & $8.3 \mathrm{E}-2$ \\
\hline SIS-MDP-EQ4-BE & SIS MDP FAILURE DUE TO SESIMIC EVENT BIN 4 & $2.9 \mathrm{E}-1$ \\
\hline SIS-MDP-EQ5-BE & SIS MDP FAILURE DUE TO SESIMIC EVENT BIN 5 & $6.0 \mathrm{E}-1$ \\
\hline SIS-MDP-EQ6-BE & SIS MDP FAILURE DUE TO SESIMIC EVENT BIN 6 & $9.4 \mathrm{E}-1$ \\
\hline SIS-MDP-FR-01A & SIS MDP-01A FAILS TO RUN & $3.9 \mathrm{E}-4$ \\
\hline SIS-MDP-FR-01B & SIS MDP-01B FAILS TO RUN & $3.9 \mathrm{E}-4$ \\
\hline SIS-MDP-FS-01A & SIS MDP-01A FAILS TO START & $7.9 \mathrm{E}-4$ \\
\hline SIS-MDP-FS-01B & SIS MDP-01B FAILS TO START & $7.9 \mathrm{E}-4$ \\
\hline SIS-MDP-TM-01A & SIS MDP-01A IS IN TEST OR MAINTENANCE & $4.2 \mathrm{E}-3$ \\
\hline SIS-MDP-TM-01B & SIS MDP-01B IS IN TEST OR MAINTENANCE & $4.2 \mathrm{E}-3$ \\
\hline SIS-MOV-CF-OOMF01 & CCF OF SIS MOV IN MINI FLOW LINE TO CLOSE & $1.7 \mathrm{E}-6$ \\
\hline SIS-MOV-OC-001A & SIS MOV-001A FAILS TO REMAIN OPEN & $7.8 \mathrm{E}-7$ \\
\hline SIS-MOV-OC-001B & SIS SUCTION MOV-001B FAILS TO REMAIN OPEN & $7.8 \mathrm{E}-7$ \\
\hline SIS-MOV-OC-002A & SIS DISCHARGE MOV-002A FAILS TO REMAIN OPEN & $7.8 \mathrm{E}-7$ \\
\hline
\end{tabular}




\section{Generic Pressurized Water Reactor (PWR)}

\begin{tabular}{|c|c|c|}
\hline Basic Event Name & Description & $\begin{array}{l}\text { Calculated } \\
\text { Probability }\end{array}$ \\
\hline SIS-MOV-OC-002B & SIS DISCHARGE MOV-002B FAILS TO REMAIN OPEN & $7.8 \mathrm{E}-7$ \\
\hline SIS-MOV-OO-MFA01 & SIS MOV IN MINI FLOW LINE-A FAILS TO CLOSE & $3.3 \mathrm{E}-4$ \\
\hline SIS-MOV-OO-MFB01 & SIS MOV IN MINI FLOW LINE-B FAILS TO CLOSE & $3.3 \mathrm{E}-4$ \\
\hline SSE-STR-HC1-CTBLD & CONTROL BUILDING STRUCTURE FAILS FROM HURRICANE BIN 1 & $3.0 \mathrm{E}-11$ \\
\hline SSE-STR-HC1-DGBLD & DIESEL GENERATOR BUILDING STRUCTURE FAILS FROM HURRICANE BIN 1 & $2.0 \mathrm{E}-12$ \\
\hline SSE-STR-HC1-RXBLD & REACTOR BUILDING STRUCTURE FAILS FROM HURRICANE BIN 1 & $1.0 \mathrm{E}-11$ \\
\hline SSE-STR-HC2-CTBLD & CONTROL BUILDING STRUCTURE FAILS FROM HURRICANE BIN 2 & $3.0 \mathrm{E}-9$ \\
\hline SSE-STR-HC2-DGBLD & DIESEL GENERATOR BUILDING STRUCTURE FAILS FROM HURRICANE BIN 2 & $2.0 \mathrm{E}-10$ \\
\hline SSE-STR-HC2-RXBLD & REACTOR BUILDING STRUCTURE FAILS FROM HURRICANE BIN 2 & $1.0 \mathrm{E}-9$ \\
\hline SSE-STR-HC3-CTBLD & CONTROL BUILDING STRUCTURE FAILS FROM HURRICANE BIN 3 & $3.0 \mathrm{E}-7$ \\
\hline SSE-STR-HC3-DGBLD & DIESEL GENERATOR BUILDING STRUCTURE FAILS FROM HURRICANE BIN 3 & $6.0 \mathrm{E}-8$ \\
\hline SSE-STR-HC3-RXBLD & REACTOR BUILDING STRUCTURE FAILS FROM HURRICANE BIN 3 & 7.0E-7 \\
\hline SSE-STR-HC4-CTBLD & CONTROL BUILDING STRUCTURE FAILS FROM HURRICANE BIN 4 & $3.0 \mathrm{E}-5$ \\
\hline SSE-STR-HC4-DGBLD & DIESEL GENERATOR BUILDING STRUCTURE FAILS FROM HURRICANE BIN 4 & $6.0 \mathrm{E}-6$ \\
\hline SSE-STR-HC4-RXBLD & REACTOR BUILDING STRUCTURE FAILS FROM HURRICANE BIN 4 & $4.0 \mathrm{E}-5$ \\
\hline SSE-STR-HWD-CTBLD & CONTROL BUILDING STRUCTURE FAILS FROM HWD & $5.0 \mathrm{E}-14$ \\
\hline SSE-STR-HWD-DGBLD & DIESEL GENERATOR BUILDING STRUCTURE FAILS FROM HWD & $2.0 \mathrm{E}-14$ \\
\hline SSE-STR-HWD-RXBLD & REACTOR BUILDING STRUCTURE FAILS FROM HWD & $1.0 \mathrm{E}-13$ \\
\hline SSE-STR-TD1-CTBLD & CONTROL BUILDING STRUCTURE FAILS FROM TORNADO BIN 1 & $3.0 \mathrm{E}-8$ \\
\hline SSE-STR-TD1-DGBLD & DIESEL GENERATOR BUILDING STRUCTURE FAILS FROM TORNADO BIN 1 & $2.0 \mathrm{E}-7$ \\
\hline SSE-STR-TD1-RXBLD & REACTOR BUILDING STRUCTURE FAILS FROM TORNADO BIN 1 & $6.0 \mathrm{E}-8$ \\
\hline SSE-STR-TD2-CTBLD & CONTROL BUILDING STRUCTURE FAILS FROM TORNADO BIN 2 & $3.0 \mathrm{E}-6$ \\
\hline SSE-STR-TD2-DGBLD & DIESEL GENERATOR BUILDING STRUCTURE FAILS FROM TORNADO BIN 2 & $2.0 \mathrm{E}-5$ \\
\hline SSE-STR-TD2-RXBLD & REACTOR BUILDING STRUCTURE FAILS FROM TORNADO BIN 2 & $6.0 \mathrm{E}-6$ \\
\hline SSE-STR-TD3-CTBLD & CONTROL BUILDING STRUCTURE FAILS FROM TORNADO BIN 3 & $3.0 \mathrm{E}-4$ \\
\hline SSE-STR-TD3-DGBLD & DIESEL GENERATOR BUILDING STRUCTURE FAILS FROM TORNADO BIN 3 & $2.0 \mathrm{E}-3$ \\
\hline SSE-STR-TD3-RXBLD & REACTOR BUILDING STRUCTURE FAILS FROM TORNADO BIN 3 & $6.0 \mathrm{E}-4$ \\
\hline STR-EQ1-CD & MAJOR STRUCTURE FAILURE DIRECT CD BIN 1 & $4.0 \mathrm{E}-9$ \\
\hline STR-EQ2-CD & MAJOR STRUCTURE FAILURE DIRECT CD BIN 2 & $5.4 \mathrm{E}-5$ \\
\hline STR-EQ3-CD & MAJOR STRUCTURE FAILURE DIRECT CD BIN 3 & $2.6 \mathrm{E}-3$ \\
\hline STR-EQ4-CD & MAJOR STRUCTURE FAILURE DIRECT CD BIN 4 & $2.4 \mathrm{E}-2$ \\
\hline STR-EQ5-CD & MAJOR STRUCTURE FAILURE DIRECT CD BIN 5 & $1.2 \mathrm{E}-1$ \\
\hline STR-EQ6-CD & MAJOR STRUCTURE FAILURE DIRECT CD BIN 6 & $5.6 \mathrm{E}-1$ \\
\hline STR-EQ7-CD & MAJOR STRUCTURE FAILURE DIRECT CD BIN 7 & $8.3 \mathrm{E}-1$ \\
\hline SWS-CKV-CC-001A & SWS-CKV-CC-001A FAILS TO OPEN & $9.2 \mathrm{E}-6$ \\
\hline SWS-CKV-CC-001B & SWS-CKV-CC-001B FAILS TO OPEN & $9.2 \mathrm{E}-6$ \\
\hline SWS-CKV-CC-001C & SWS-CKV-CC-001C FAILS TO OPEN & $9.2 \mathrm{E}-6$ \\
\hline SWS-CKV-CF-CC001 & CCF OF SWS CKV-001 TO OPEN & $6.1 \mathrm{E}-8$ \\
\hline SWS-MDP-CF-FTR & CCF OF SWS MDP TO RUN & $4.0 \mathrm{E}-7$ \\
\hline SWS-MDP-CF-FTS & CCF OF SWS MDP TO START & $3.7 \mathrm{E}-6$ \\
\hline SWS-MDP-EQ1-BE & SWS MDP FAILURE DUE TO SESIMIC EVENT BIN 1 & $6.5 \mathrm{E}-6$ \\
\hline SWS-MDP-EQ2-BE & SWS MDP FAILURE DUE TO SESIMIC EVENT BIN 2 & $6.9 \mathrm{E}-3$ \\
\hline SWS-MDP-EQ3-BE & SWS MDP FAILURE DUE TO SESIMIC EVENT BIN 3 & $8.3 \mathrm{E}-2$ \\
\hline SWS-MDP-EQ4-BE & SWS MDP FAILURE DUE TO SESIMIC EVENT BIN 4 & $2.9 \mathrm{E}-1$ \\
\hline SWS-MDP-EQ5-BE & SWS MDP FAILURE DUE TO SESIMIC EVENT BIN 5 & $6.0 \mathrm{E}-1$ \\
\hline SWS-MDP-EQ6-BE & SWS MDP FAILURE DUE TO SESIMIC EVENT BIN 6 & $9.4 \mathrm{E}-1$ \\
\hline SWS-MDP-FR-1A & SWS MOTOR DRIVEN PUMP 1A FAILS TO RUN & $1.6 \mathrm{E}-4$ \\
\hline SWS-MDP-FR-1B & SWS MOTOR DRIVEN PUMP 1B FAILS TO RUN & $1.6 \mathrm{E}-4$ \\
\hline SWS-MDP-FR-1C & SWS MOTOR DRIVEN PUMP 1C FAILS TO RUN & $1.6 \mathrm{E}-4$ \\
\hline SWS-MDP-FS-1A & SWS MOTOR DRIVEN PUMP 1A FAILS TO START & $8.4 \mathrm{E}-4$ \\
\hline SWS-MDP-FS-1B & SWS MOTOR DRIVEN PUMP 1B FAILS TO START & $8.4 \mathrm{E}-4$ \\
\hline SWS-MDP-FS-1C & SWS MOTOR DRIVEN PUMP 1C FAILS TO START & $8.4 \mathrm{E}-4$ \\
\hline
\end{tabular}




\section{Generic Pressurized Water Reactor (PWR)}

\begin{tabular}{|l|l|l|}
\hline \multicolumn{1}{|c|}{ Basic Event Name } & & \multicolumn{1}{|c|}{ Description } \\
Probabilated \\
SWS-MDP-TM-1A & SWS MDP-1A IN TEST AND MAINTENANCE & $4.2 \mathrm{E}-3$ \\
\hline SWS-MDP-TM-1B & SWS MDP-1B IN TEST AND MAINTENANCE & $4.2 \mathrm{E}-3$ \\
\hline SWS-MDP-TM-1C & SWS MDP-1C IN TEST AND MAINTENANCE & $4.2 \mathrm{E}-3$ \\
\hline SWS-MOV-OC-001A & SWS MDP-C CROSS-TIE MOV TO TRAIN A & $7.8 \mathrm{E}-7$ \\
\hline SWS-MOV-OC-001B & SWS MDP-C CROSS-TIE MOV TO TRAIN B & $7.8 \mathrm{E}-7$ \\
\hline SWS-STR-CF-PG & CCF OF SWS STRAINER PLUGGING & $6.6 \mathrm{E}-7$ \\
\hline SWS-STR-HC1-STRC & SWS STRAINER STRUCTURE FAILURE DUE TO HURRICANE BIN 1 & $3.0 \mathrm{E}-10$ \\
\hline SWS-STR-HC2-STRC & SWS STRAINER STRUCTURE FAILURE DUE TO HURRICANE BIN 2 & $4.0 \mathrm{E}-8$ \\
\hline SWS-STR-HC3-STRC & SWS STRAINER STRUCTURE FAILURE DUE TO HURRICANE BIN 3 & $5.0 \mathrm{E}-6$ \\
\hline SWS-STR-HC4-STRC & SWS STRAINER STRUCTURE FAILURE DUE TO HURRICANE BIN 4 & $6.0 \mathrm{E}-4$ \\
\hline SWS-STR-HWD-STRC & SWS STRAINER STRUCTURE FAILURE DUE TO HIGH WIND & $2.0 \mathrm{E}-12$ \\
\hline SWS-STR-PG-1A & SWS STRAINER 1A IS UNAVAILABLE & $3.3 \mathrm{E}-5$ \\
\hline SWS-STR-PG-1B & SWS STRAINER 1B IS UNAVAILABLE & $3.3 \mathrm{E}-5$ \\
\hline SWS-STR-PG-1C & SWS STRAINER 1C IS UNAVAILABLE & $3.3 \mathrm{E}-5$ \\
\hline SWS-STR-TD2-STRC & SWS STRAINER STRUCTURE FAILURE DUE TO TORNADO BIN 2 & $5.0 \mathrm{E}-6$ \\
\hline SWS-STR-TD3-STRC & SWS STRAINER STRUCTURE FAILURE DUE TO TORNADO BIN 3 & $6.0 \mathrm{E}-4$ \\
\hline SWS-TRN-OP-STDYA & SWS PUMP-A TRAIN IS STANDBY & $5.0 \mathrm{E}-1$ \\
\hline SWS-TRN-OP-STDYB & SWS PUMP-B TRAIN IS STANDBY & $5.0 \mathrm{E}-1$ \\
\hline SWS-XHE-XM-TRNC & OPERATOR FAILS TO START AND ALIGN SWS TRAIN C & $1.0 \mathrm{E}-3$ \\
\hline SWS-XVM-CO-002B & SWS XVM-002B FAILS TO REMAIN CLOSE & $1.2 \mathrm{E}-6$ \\
\hline SWS-XVM-OO-002A & SWS XVM-002A FAILS TO CLOSE & $4.6 \mathrm{E}-4$ \\
\hline TOR-SWY-LP-SWYRD-1 & SWITCHYARD FAILURE DUE TO TORNADO WIND BIN 1 & $1.0 \mathrm{E}-2$ \\
\hline TOR-SWY-LP-SWYRD-2 & SWITCHYARD FAILURE DUE TO TORNADO WIND BIN 2 & $1.0 \mathrm{E}-1$ \\
\hline TOR-SWY-LP-SWYRD-3 & SWITCHYARD FAILURE DUE TO TORNADO WIND BIN 3 & $5.0 \mathrm{E}-1$ \\
\hline ZV-FALSE & & $1.0 \mathrm{E}+0$ \\
\hline
\end{tabular}

